\title{
GREEN SOLVENT-ENABLED BIOMASS CONVERSION FOR RENEWABLE CHEMICALS AND LIGNIN UPGRADING
}

A Dissertation
presented to
the Faculty of the Graduate School
at the University of Missouri-Columbia
In Partial Fulfillment
of the Requirements for the Degree
Doctor of Philosophy
by
Zhu Chen
Dr. Caixia Wan, Dissertation Advisor
December 2018


The undersigned, appointed by the dean of the Graduate School, have examined the dissertation entitled

\section{GREEN SOLVENT-ENABLED BIOMASS CONVERSION FOR RENEWABLE CHEMICALS AND LIGNIN UPGRADING}

presented by Zhu Chen,

a candidate for the degree of Doctor of Philosophy of Biological Engineering, and hereby certify that, in their opinion, it is worthy of acceptance.

Dr. Caixia Wan, Bioengineering

Dr. William A. Jacoby, Bioengineering

Dr. Shramik Sengupta, Bioengineering

Dr. C. Michael Greenlief, Chemistry

Dr. Gary A. Baker, Chemistry 


\section{ACKNOWLEDGEMENTS}

Firstly, I would like to express my special appreciation to my advisor Dr. Caixia Wan. She gives me enough freedom and support to explore new ideas, and provides insightful discussion and valuable suggestions about the research projects. Without her guidance and support, this thesis would not have been finished.

Besides my advisor, I would also like to thank my other committee members: Dr. William A. Jacoby, Dr. Shramik Sengupta, Dr. C. Michael Greenlief, and Dr. Gary A. Baker. Their invaluable advices helped me to think about the research projects from different perspectives, and come up with new ideas on my projects.

My sincere thanks also go to Lakdas Fernando, Dr. Wycoff Wei and Dr. Xianglan Bai (Iowa State University) for their kind help for doing sample analysis. I am also very grateful to the help from the current and past lab mates: Safoora Mirmohamadsadeghi, Maryam Al Ameri, Ubaldo Abrego, Ying Wu, Haoqi Wang, Xin Sun, Yisheng Sun, Hanwen Zhang, Wesley D Reznicek, and Faisal Mahmood. Their great help made my project go more smoothly.

Finally, I would like to thank my family for their love and support. Without their understanding and encouragement, this dissertation would not have been possible. 


\section{TABLE OF CONTENTS}

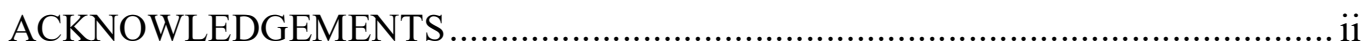

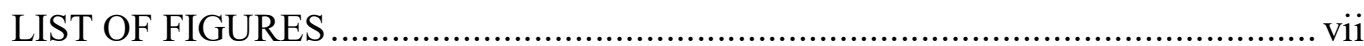

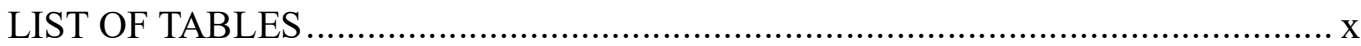

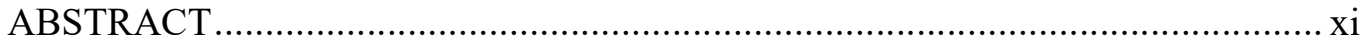

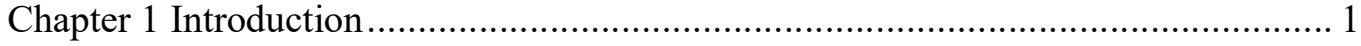

1.1. Lignocellulosic biomass compositions................................................... 2

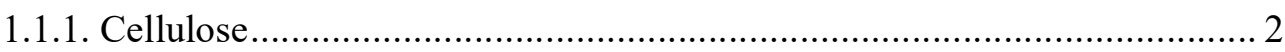

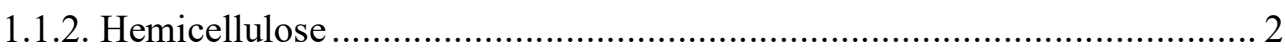

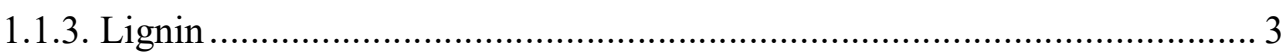

1.2. Pretreatment methods ...................................................................... 4

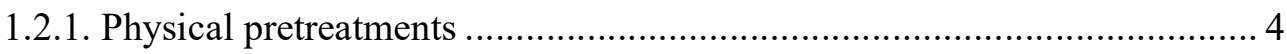

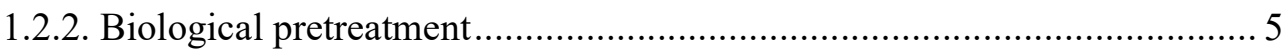

1.2.3. Thermal pretreatment..................................................................... 5

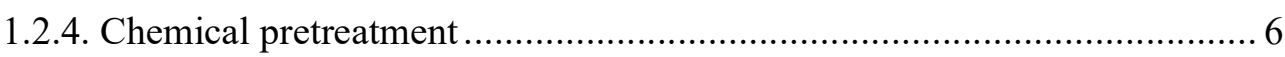

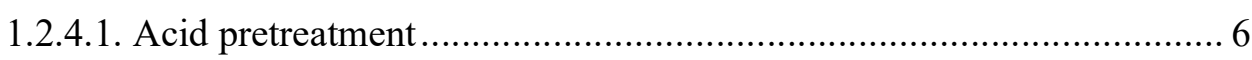

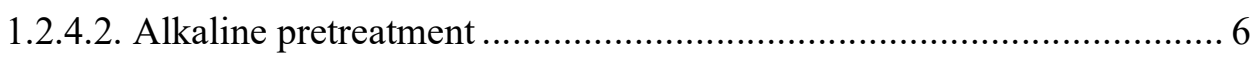

1.2.4.3. Organic solvents ........................................................................ 7

1.2.4.4. Ionic liquids (ILs) ................................................................. 7

1.2.4.5. Deep eutectic solvents (DESs) ................................................. 8

1.4. Limitations of current pretreatment ......................................................... 8

1.5. Uniqueness of using QAS based solvents ............................................... 9

1.5.1. High delignification and production of valorizable lignin...................... 9

1.5.2. Significantly enhanced cellulose digestibility ..................................... 11

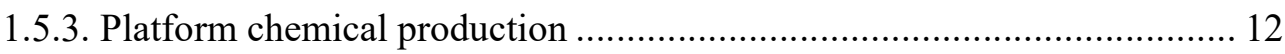

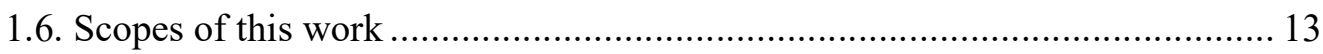

Chapter 2 A Tunable Platform Deep Eutectic Solvent for the Integrated Biorefinery 15

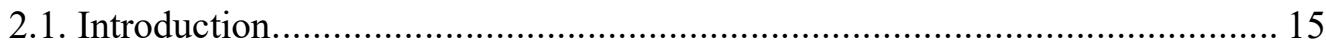

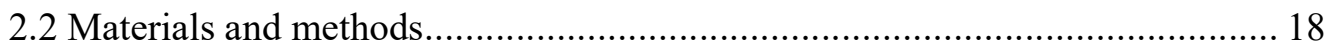

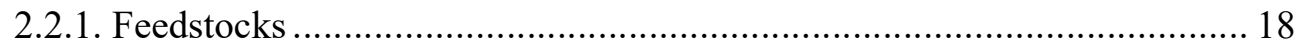

2.2.2. DES Synthesis, pretreatment and lignin recovery ................................ 19

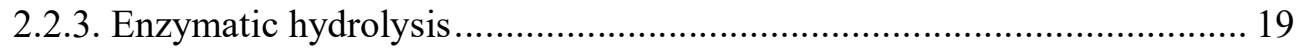

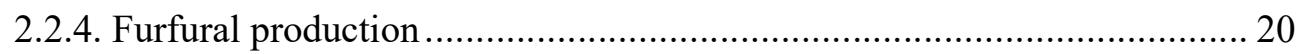

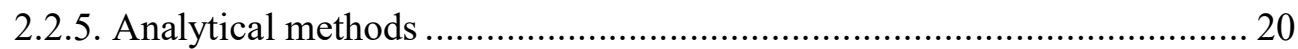

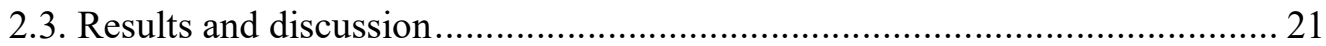


2.3.1. Characterization of pretreated switchgrass ....................................... 21

2.3.2. Sugar production via high solid loading enzymatic hydrolysis ................ 25

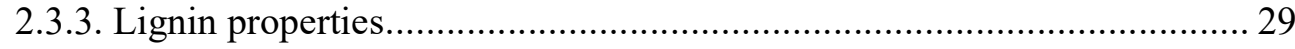

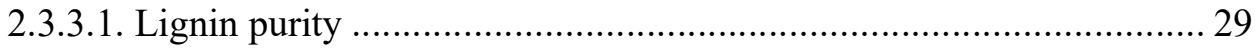

2.3.3.2. 2D HSQC NMR analysis of lignin.............................................. 33

2.4. Furfural production from pretreatment liquor ........................................... 39

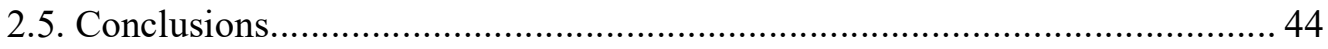

Chapter 3 High-solid Lignocellulose Processing Enabled by Natural Deep Eutectic

Solvent for Lignin Extraction and Industrially Relevant Production of Renewable Chemicals. 46

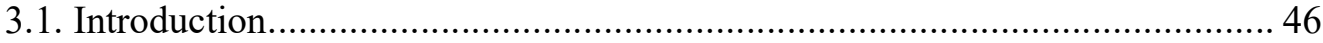

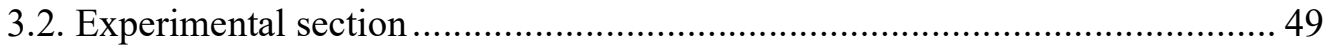

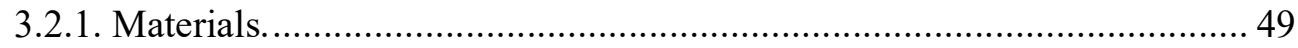

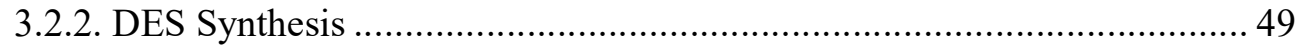

3.2.3. DES pretreatment and lignin recovery............................................... 49

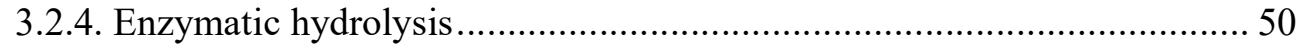

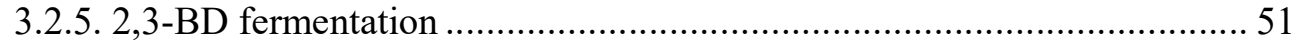

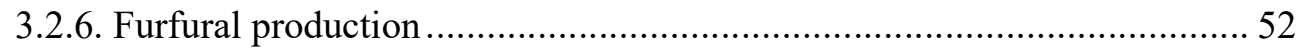

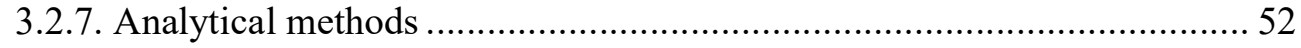

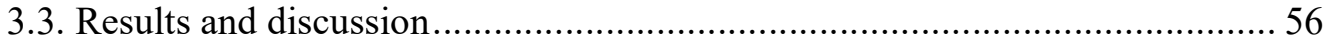

3.3.1. Effects of acidification on the pretreatment performance of $\mathrm{ChCl}: \mathrm{EG}$..... 56

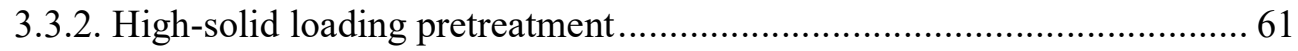

3.3.3. High solid loading enzymatic hydrolysis ............................................. 66

3.3.4. 2,3-BD production from concentrated sugar hydrolysate ........................ 69

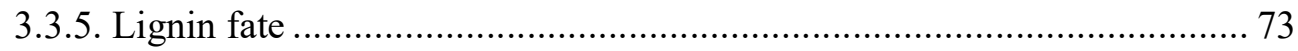

3.3.6. Pretreatment liquor upgrading for furfural production .......................... 83

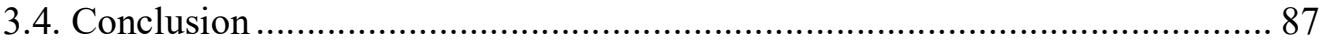

Chapter 4 Ternary Deep Eutectic Solvents for Switchgrass Fractionation................. 88

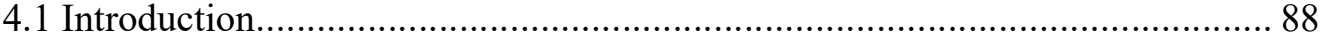

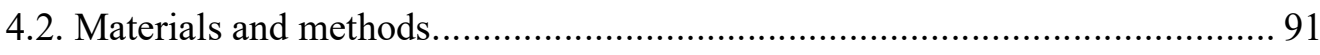

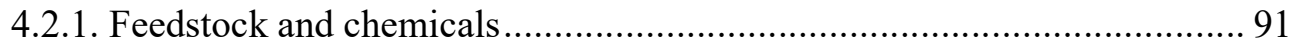

4.2.2. Syntheis of ternary DESs ............................................................. 91

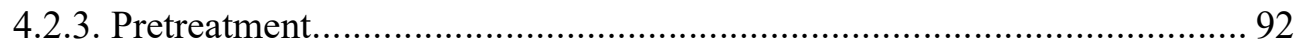

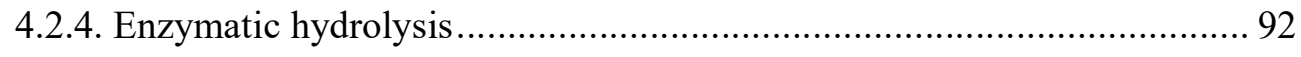

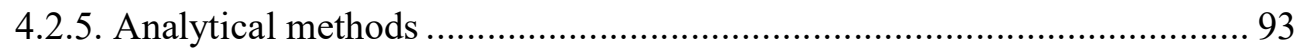

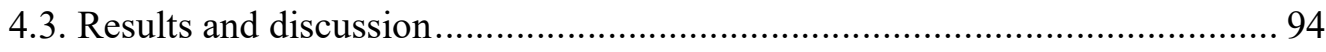


4.3.1. Screening effective ternary DESs for pretreatment ............................. 94

4.3.2. Lignin characterization by NMR ........................................................ 97

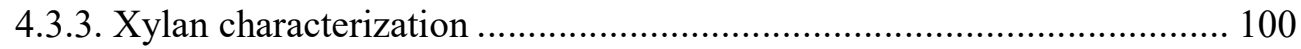

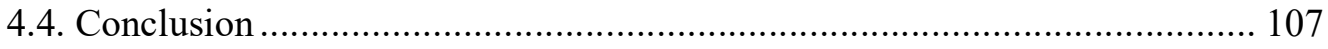

Chapter 5 Aqueous Choline Chloride: a Novel Solvent for Switchgrass Fractionation and Subsequent Hemicellulose Conversion into Furfural ................................... 109

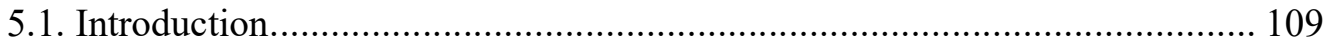

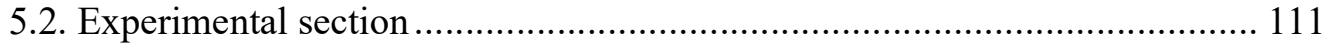

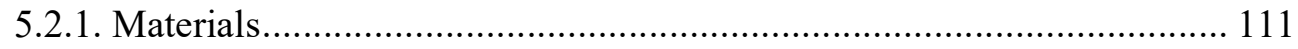

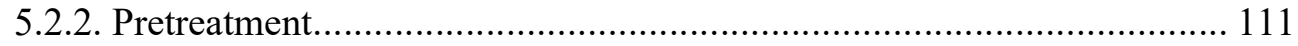

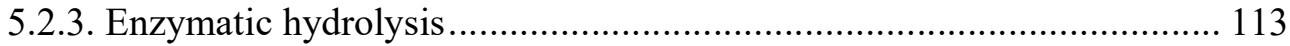

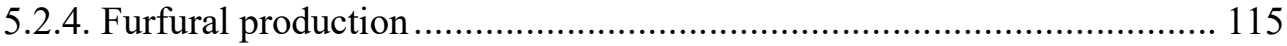

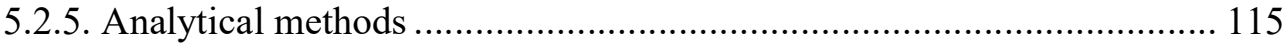

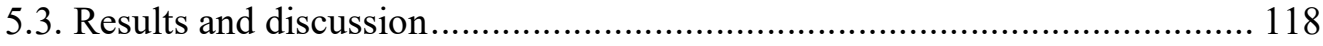

5.3.1. Comparison of pretreatment effectiveness of ChCl-based solvents ........ 118

5.3.2. Recyclability and reusability of acidified $\mathrm{ChCl}$ solution ...................... 120

5.3.3. Digestibility of pretreated switchgrass............................................. 125

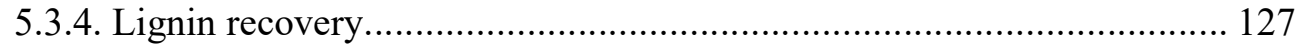

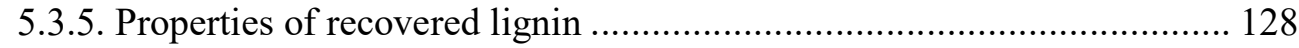

5.3.6. Xylose-rich pretreatment liquor used for furfural production................ 130

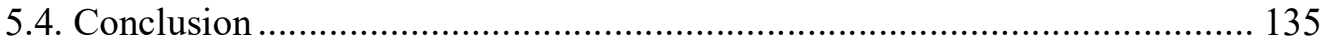

Chapter 6 Aqueous Choline Chloride Enables Biomass Fractionation and Conversion

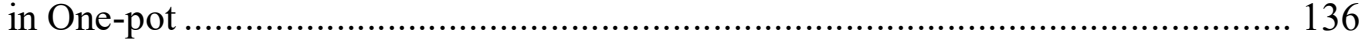

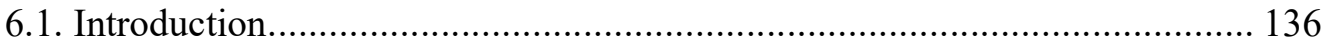

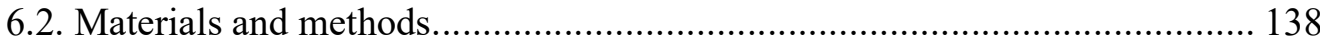

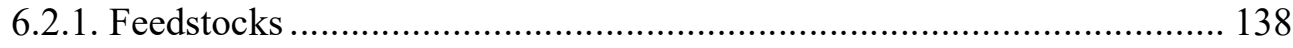

6.2.2. One-pot furfural production from switchgrass ................................... 139

6.2.3. One-pot furfural production using recycled solvent ............................ 140

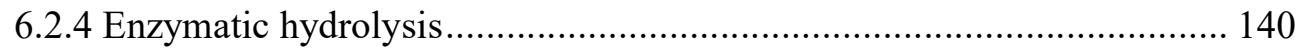

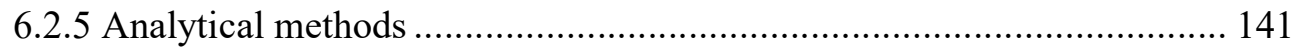

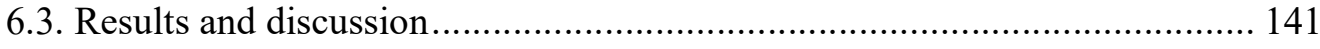

6.3.1 Effects of reaction conditions on furfural production and compositional

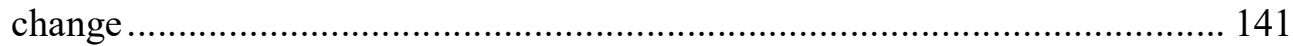

6.3.2. Comparison of performance between aqueous $\mathrm{ChCl} / \mathrm{MIBK}$ and $\mathrm{H}_{2} \mathrm{O} / \mathrm{MIBK}$ 145

6.3.3. Lignin characterization 147 


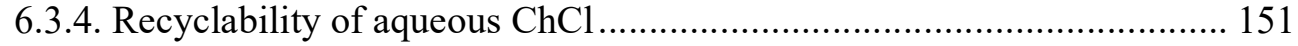

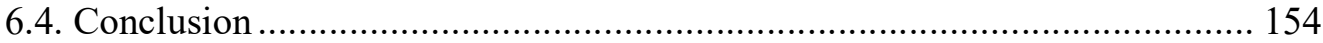

Chapter 7 Conclusions and suggested future work ........................................... 155

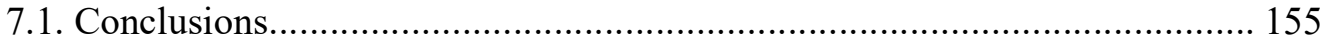

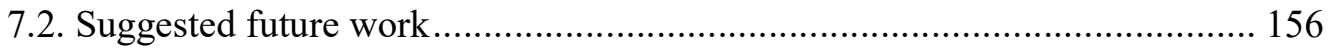

7.2.1. Understand the mechanism for $\beta-O-4$ linkage stabilization by choline

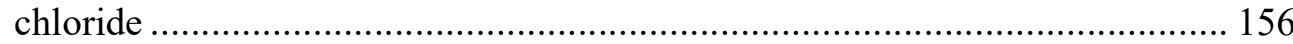

7.2.2. Lignin upgrading into value-added products...................................... 156

7.2.3. Cellulose-based advanced materials production.................................. 157

7.2.4. One-pot production of jet-fuel precursors from lignocellulosic biomass 157

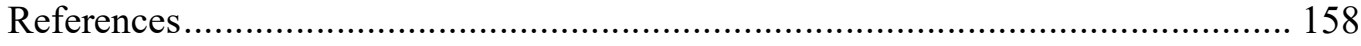

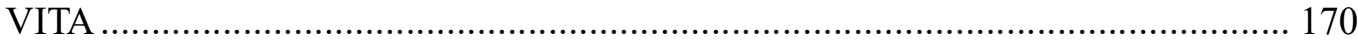




\section{LIST OF FIGURES}

Figure 1.1 Structure of lignocellulosic biomass.................................................. 2

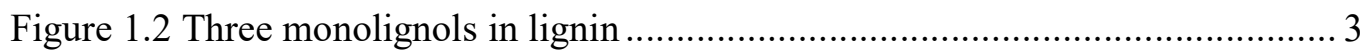

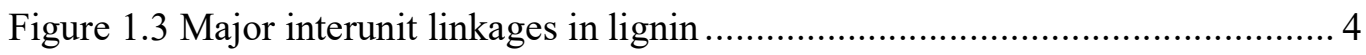

Figure 2.1 Switchgrass component distribution and solid recovery upon pretreatment.

Figure 2.2 Switchgrass component removal upon pretreatment............................. 24

Figure 2.3 Digestibility of pretreated switchgrass upon pretreatment. .................... 27

Figure 2.4 Monomeric sugar concentration in the hydrolysates............................ 28

Figure 2.5 Pretreated switchgrass using acidified $\left(1 \% \mathrm{H}_{2} \mathrm{SO}_{4}\right)$ neat $\mathrm{ChCl}: \mathrm{EG}(1: 2)$

(left ) and aqueous $\mathrm{ChCl}: \mathrm{EG}(1: 2)$ (right) under $130{ }^{\circ} \mathrm{C}$ for $30 \mathrm{~min} . \ldots \ldots \ldots \ldots \ldots . . . . . .28$

Figure 2.6 Illustration of acid catalyzed cleavage of $\beta-\mathrm{O}-4$ linkage in lignin. ........... 32

Figure 2.7 Proposed reaction of EG with depolymerized lignin fraction under acidic

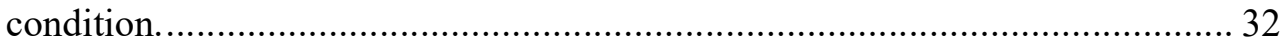

Figure 2.8 2D HSQC NMR spectra of lignin. L1-12 represent lignin samples listed in Table 2.1

Figure 2.9 Stabilization of carbocation intermediate by $\mathrm{Cl}-1$ in acid catalyzed

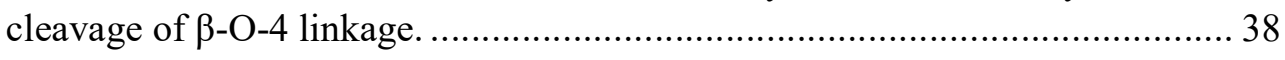

Figure 2.10 Phase formation between different DESs and acetone. ...................... 40

Figure 2.11 Partition coefficient for furfural in aqueous ChCl:EG(1:2)/Acetone

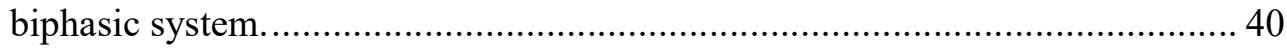

Figure 2.12 Kinetics of furfural production in aqueous $\mathrm{ChCl}$ :EG/Acetone biphasic

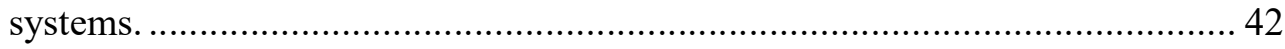

Figure 2.13 Effects of acetone to aqueous $\mathrm{ChCl}$ :EG ration on furfural production.... 42

Figure 2.14 Effects of $\mathrm{AlCl}_{3}$ loading on furfural production.................................. 43

Figure 2.15 Furfural production in pretreatment liquor/acetone biphasic system. .... 44

Figure 3.1 Switchgrass component removal in response to the pretreatment using different solvents.

Figure 3.2 Switchgrass major component distribution and solid recovery in response to the pretreatment using different solvents. ........................................... 57

Figure 3.3 XRD pattern of pretreated switchgrass............................................. 59

Figure 3.4 FTIR spectra of pretreated switchgrass............................................... 59

Figure 3.5 Switchgrass digestibility in response to the pretreatment using different solvents.

Figure 3.6 Switchgrass component removal and digestibility in response to $\mathrm{ChCl}$ :EG pretreatment under acidic condition with varying water addition and solid loadings. 
Figure 3.7 Switchgrass major component distribution and solid recovery in response to the pretreatment using acidified $\mathrm{ChCl}$ :EG with a varying water addition and solid loading.

Figure 3.8 Switchgrass digestibility in response to $\mathrm{ChCl}$ :EG pretreatment under acidic condition with varying water addition and solid loadings.

Figure 3.9 Switchgrass component removal in response to different pretreatment cycles under acidic condition using aqueous $\mathrm{ChCl}: \mathrm{EG}$ with $10 \%$ water and at $27 \%$ solid loading.

Figure 3.10 Switchgrass component distribution in response to different pretreatment cycles under acidic condition using aqueous $\mathrm{ChCl}$ :EG with $10 \%$ water and at $27 \%$ solid loading

Figure 3.11 Switchgrass digestibility in response to different pretreatment cycles under acidic condition using aqueous $\mathrm{ChCl}: \mathrm{EG}$ with $10 \%$ water and at $27 \%$ solid loading. 66

Figure 3.12 High-solid loading enzymatic hydrolysis of switchgrass pretreated at high solids using acidified, aqueous $\mathrm{ChCl}$ :EG with $10 \%$ water. 68

Figure 3.13 Fermentative production of 2,3-BD from switchgrass hydrolysate of $\mathrm{P} 20 / \mathrm{EH} 20$.

Figure 3.14 Fermentative production of 2,3-BD from switchgrass hydrolysate of $\mathrm{P} 20 / \mathrm{EH} 25$. 70

Figure 3.15 Mass flow analysis of the proposed biorefinery based on high-solid loading DES pretreatment for lignocellulose conversion into platform chemicals.

Figure 3.16 2D HSQC NMR analysis of DES lignin........................................ 78

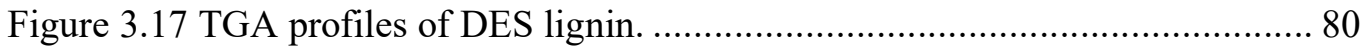

Figure 3.18 Effect of $\mathrm{AlCl} 3$ addition on $100 \mathrm{~g} / \mathrm{L}$ xylose conversion into furfural. .... 86

Figure 3.19 Furfural production from the pretreatment liquor. .............................. 86

Figure 4.1Component removal of switchgrass upon ternary DESs pretreatment....... 95

Figure 4.2 Component distribution and solid recovery of switchgrass upon ternary

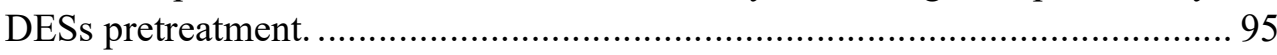

Figure 4.3 Digestibility of switchgrass pretreated by different ternary DESs........... 96

Figure 4.4 2D HSQC NMR spectra of lignin...................................................... 98

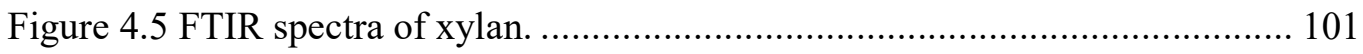

Figure $4.6{ }^{13} \mathrm{C}$ NMR spectra of pristine xylan and treated xylan........................... 101

Figure 4.7 2D HSQC NMR spectra of pristine xylan and treated xylan ................ 102

Figure 4.8 HPLC chromatograph of CC-EG-PTSA with xylan dissolved.............. 102

Figure 4.9 Proposed mechanisms for switchgrass fractionation in ternary DESs. ... 103

Figure 4.10 Component removal of switchgrass after high solid loading pretreatment. 
Figure 4.11 Component distribution and solid recovery of switchgrass after high solid loading pretreatment.

Figure 4.12 Digestibility of switchgrass and sugar concentration from $20 \%(\mathrm{w} / \mathrm{w})$ solid loading enzymatic hydrolysis

Figure 5.1 Schematic diagram of the proposed biomass pretreatment and furfural production process.

Figure 5.2 XRD pattern of the pretreated switchgrass from different pretreatment cycle.

Figure 5.3 FTIR spectra of pretreated switchgrass from different pretreatment cycles.

Figure 5.4 Digestibility of switchgrass pretreated by different solvents. 126

Figure 5.5 Digestibility of the pretreated switchgrass from different pretreatment cycles.

Figure 5.6 Lignin removal and yield resulting from all the pretreatment cycles...... 128

Figure 5.7 2D HSQC NMR spectra of lignin.

Figure 5.8 Effects of reaction temperature on furfural production from xylose in acidified $\mathrm{ChCl}$ solution.

Figure 5.9 Effects of reaction time on furfural production from xylose in acidified $\mathrm{ChCl}$ solution

Figure 5.10 Effects of $\mathrm{AlCl}_{3}$ on furfural production from xylose in acidified $\mathrm{ChCl}$ solution.

Figure 6.1 Schematic of the one-pot process

Figure 6.2 Furfural titer and yield in MIBK under different conditions 143

Figure 6.3 Furfural titer and yield from aqueous $\mathrm{ChCl} / \mathrm{MIBK}$ and $\mathrm{H}_{2} \mathrm{O} / \mathrm{MIBK}$ in onepot process 146

Figure 6.4 Digestibility of cellulose pulp recovered from one-pot process 147

Figure 6.5 2D HSQC NMR spectra of lignin. L-1 and L-2 represent lignin recovered from one-pot process and enzymatic hydrolysis.

Figure 6.6 Furfural concentration and yield in MIBK during different cycles.

Figure 6.7 Compositional distribution of pretreated switchgrass during different cycles.

Figure 6.8 Digestibility of switchgrass from different cycles of one-pot treatment. 153

Figure 6.9 Lignin removal and yield in different cycles 153 


\section{LIST OF TABLES}

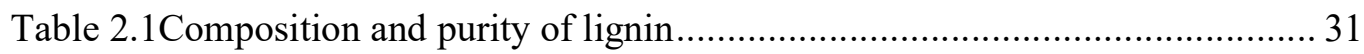

Table 2.2 Volume integration of $\beta$-O-4 interlinkages and ferulate in lignin .............. 38

Table 3.1Summary of 2,3-BD production from lignocellulosic biomass.................. 71

Table 3.2 Lignin removal and recovery in response to high-solid loading DES pretreatment with a varying water content ............................................. 75

Table 3.3 Lignin removal and recovery in response to pretreatment solvent recycling 75

Table 3.4 Semi-quantification of the $\beta-\mathrm{O}-4$ and hydroxylcinnamates abundance in lignin.

Table 3.5 Average molecular weight and polydispersity (PD) of DES lignin........... 79

Table 3.6 Yields of phenolic monomers produced from lignin pyrolysis. ................. 82

Table 3.7 Xylan removal and recovery in pretreatment liquor for furfural production.

Table 4.1 Volume integration of the $\beta-\mathrm{O}-4, \mathrm{FA}, p$-CA, S and $\mathrm{G}$ units in lignin ........ 98

Table 5.1 Compositional change of switchgrass in response to the pretreatments using different solvents

Table 5.2 Compositional changes of switchgrass in response to the recyclability of

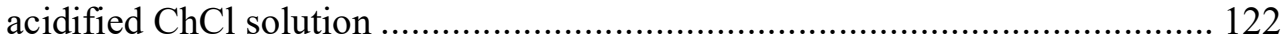

Table 5.3 Relative peak intensity of lignin and carbohydrates 125

Table 6.1 Component distribution and removal of switchgrass upon pretreatment under different conditions. 


\begin{abstract}
Lignocellulosic biomass, which is mainly composed of cellulose, hemicellulose, and lignin, is a promising feedstock for producing renewable chemicals and fuels. To make lignocellulosic biomass-based refinery (biorefinery) competitive with petroleum refinery, maximizing the utilization of the three major components is critical. This requires the efficient fractionation of lignocellulose into different streams amenable to further upgrading. To this end, this work investigated the use of quaternary ammonium salt-based green solvents for lignocellulose fractionation and upgrading into renewable chemicals.
\end{abstract}

First, a platform solvent system based on choline chloride and ethylene glycol was developed for switchgrass fractionation. Tailor-made lignin can be produced by using this platform solvent system, while the delignification and cellulose digestibility were not compromised. Highly concentrated sugar hydrolysate from pulp (cellulose) streams can be obtained via high solid loading enzymatic hydrolysis. The hemicellulose streams can be facilely upgraded into furfural via a novel biphasic system - acetone and aqueous choline chloride: ethylene glycol (ChCl:EG).

Secondly, switchgrass can be fractionated effectively at high solid loading using either aqueous $\mathrm{ChCl}: \mathrm{EG}$ or a series of ternary deep eutectic solvents. The cellulose stream can be converted into highly concentrated hydrolysate for high titer platform chemical production via fermentation. The hemicellulose and lignin streams can also be converted into other value-added products via other upgrading pathways.

Thirdly, aqueous $\mathrm{ChCl}$ can also be a good solvent for switchgrass fractionation at mild conditions. The resultant lignin presented a very similar structure to native lignin, and thus, a great potential to be valorized into different products. The 
hemicellulose and cellulose streams can also be converted into valuable chemicals via biological and chemical routes.

Lastly, a one-pot process capable of directly converting the untreated lignocellulosic biomass into furfural, digestible cellulose pulp and high purity lignin was developed. Furfural can be produced with high yield, while cellulose and lignin with appealing properties can be obtained via a one-pot process. 


\section{Chapter 1 Introduction}

The depletion of fossil fuels and environmental issues arising from the consumption of fossils fuels (e.g., greenhouse effects, air pollution) have driven the modern society to seek for alternative feedstocks for chemical and fuel production. Lignocellulosic biomass is an abundant, widely available, and renewable resource on earth, and can be converted into biofuels and chemicals via different routes. These unique features spawned the exploration of using lignocellulosic biomass as feedstock for producing chemicals and fuels with similar properties to their counterparts from petroleum refinery. Various renewable products, such as ethanol and butanol, have been successfully produced from lignocellulosic biomass at industrial scale.

One common strategy to convert lignocellulosic biomass into chemicals and fuels is to first enzymatically hydrolyze the polysaccharide into monomeric sugars (e.g., glucose, xylose) as this is a waste-less and low-energy input process (Haghighi Mood et al., 2013). However, the recalcitrance of lignocellulosic biomass is the major barrier for efficient sugar conversion. To achieve better hydrolysis, a pretreatment is still necessary to deconstruct the complicated structure of lignocellulosic biomass. The deconstructed lignocellulose often renders an improvement of digestibility of cellulose and/or hemicellulose. During the past decades, different pretreatment has been developed to enhance sugar conversion from lignocellulose. However, to make biorefinery competitive with petroleum refinery, it is important to maximize the utilization of lignocellulose and increasing the revenue from it. Therefore, there is still a need to develop an economically and environmentally viable pretreatment to fractionate lignocellulose into different streams amenable for further upgrading (Sun et al., 2015). 


\subsection{Lignocellulosic biomass compositions}

\subsubsection{Cellulose}

Cellulose is a polysaccharide with repeated D-glucose linked by $\beta-(1,4)$ glycosidic bonds (Figure 1.1) (Haghighi Mood et al., 2013). Depending on biomass types, cellulose accounts for 30 to $50 \%$ of the total lignocellulosic biomass (Mosier et al., 2005). Cellulose can be hydrolyzed into glucose by cellulase. Since it is the most abundant component in lignocellulosic biomass, and its derived monomeric sugar (i.e., glucose) is a preferable carbon source for many microorganisms, most of prior work on lignocellulosic biomass conversion was focused on converting cellulose into biofuels (e.g., ethanol, butanol) and chemicals.

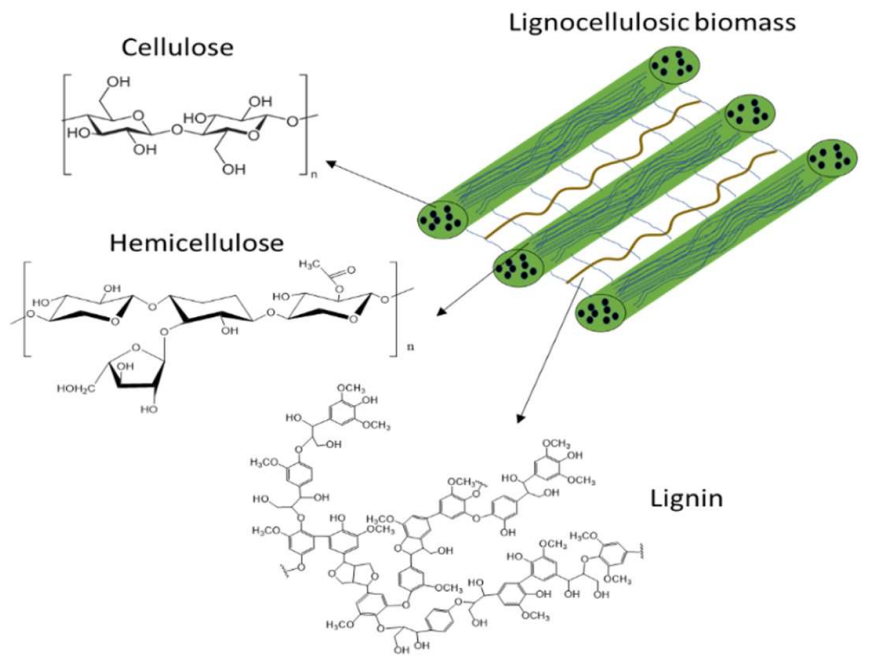

Figure 1.1 Structure of lignocellulosic biomass

\subsubsection{Hemicellulose}

Hemicellulose has a complex carbohydrate structure that consists of sugarsbased molecules such as pentoses (e.g., xylose and arabinose), hexoses (e.g., mannose, glucose and galactose), and sugar acids. Hemicellulose composition varies from one plant species to another. For example, in hardwood and grasses, xylan is the dominant hemicellulose, while glucomannan is the dominant for softwood. The 
cellulose fibrils are often covered by hemicellulose, which blocks the access of enzymes to them. Therefore, a lot of developed pretreatments aim at removing hemicellulose, which can then be depolymerized into monomeric sugars, such as xyose and arabinose. Such monomeric sugars can be upgraded into a variety of chemicals and fuels.

\subsubsection{Lignin}

Lignin is present in the primary cell wall, and provides mechanical strength and protection to the plant cell (Kumar et al., 2009). It is a heterogeneous biopolymer with monolignols, i.e., $p$-coumaryl alcohol, coniferyl alcohol, and sinapyl alcohol (Figure 1.2), as the building blocks, which are interlinked by different bonds (Figure 1.3). The major bonds found in lignocellulosic biomass are $\beta-\mathrm{O}-4,5-5, \beta-5,4-\mathrm{O}-5, \beta-$ 1 , and $\beta-\beta$ linkages, with $\beta-O-4$ linkage the most dominant one (Sen et al., 2015). Compared with cellulose and hemicellulose, lignin possesses a more irregular structure due to the existence of different linkages and building blocks. Due to its high heterogeneity and recalcitrance, lignin generated from biorefinery is generally considered as a waste, and often burned as a low quality solid fuel for energy supply to biorefinery facilities.

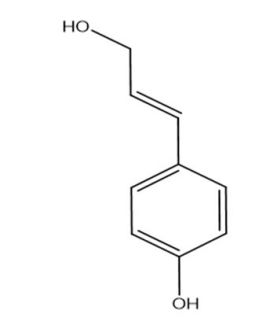

p-Coumaryl alcohol(H)

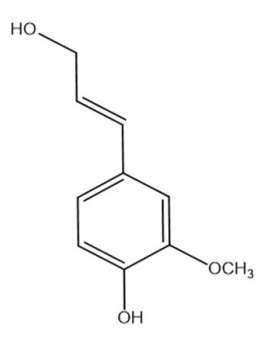

Coniferyl alcohol(G)

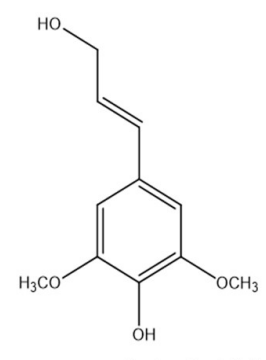

Sinapyl alcohol (S)

Figure 1.2 Three monolignols in lignin 

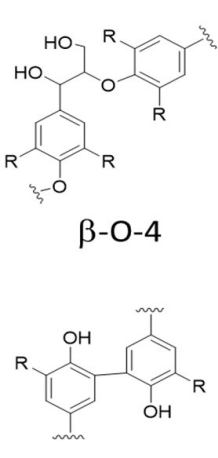

$5-5^{\prime}$
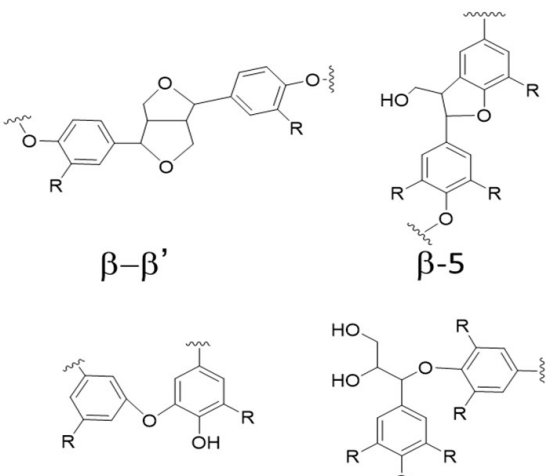

$5-\mathrm{O}-4$

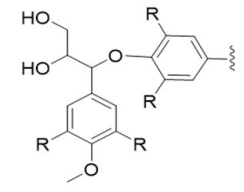

$\alpha-\mathrm{O}-4$

Figure 1.3 Major interunit linkages in lignin

\subsection{Pretreatment methods}

To improve cellulose conversion into sugars through enzymatic hydrolysis, improving the access of cellulose to cellulase via pretreatment is often necessary. The concerted efforts made by researchers in the past several decades have led to the development of many effective pretreatment.

\subsubsection{Physical pretreatments}

Physical pretreatment disrupts the structure of lignocellulsic biomass without using chemicals or microorganisms (Zheng et al., 2014). The physical pretreatment developed so far includes mechanical comminution (e.g., chipping, grinding, milling) and irradiation (e.g., gamma rays, microwave) (Zheng et al., 2014). Comminution mainly aims at reducing the particle size of lignocellulosic biomass, and is often applied before other pretreatment steps. One major advantage of comminution is no formation of inhibitory compounds, but the apparent disadvantage is high energy consumption. In terms of irradiation, it alone is often not effective to disrupt lignocellulosic biomass, and therefore, is commonly used as an assistive method to enhance the performance of other pretreatments (Binod et al., 2012; Hu \& Wen, 2008). Microwave is a commonly used irradiation due to its easy availability. 
Compared with traditional heating assisted pretreatment, microwave-based pretreatment is more energy efficient (Gong et al., 2010). However, the scale up of irradiation pretreatment is still difficult.

\subsubsection{Biological pretreatment}

In nature, many microorganisms (e.g., bacteria, fungus) have evolved the degradation enzymes to deconstruct lignocellulosic biomass. Compared with bacteria, fungi often have a more complex degradation system, and are more commonly used for lignocellulosic biomass pretreatment. Certain fungi secret a series of lignin degrading enzymes, such as lignin peroxidase (LiP), manganese peroxidase (MnP), and laccase, to depolymerize lignin into lower molecular weight compounds (Wan \& Li, 2012). Some fungi (e.g., Ceriporiopsis subvermispora, Punctularia sp. ) selectively degrade lignin while remaining polysaccharides less attacked (Suhara et al., 2012). These fungi have been commonly used for biological pretreatment due to selective removal of lignin. Biological pretreatment features ecological friendliness and almost no generation of inhibitory compounds, but the downside of this pretreatment is long pretreatment time.

\subsubsection{Thermal pretreatment}

Thermal pretreatment refers to the pretreatment in which lignocellulosic biomass is heated at high temperature (usually $150-180^{\circ} \mathrm{C}$ ) and fractionated without adding additional chemicals. Steam explosion and liquid hot water pretreatment are the two most common thermal pretreatment. During steam explosion pretreatment, lignocellulosic biomass is treated with high-pressure saturated steam at high temperature for a few minute, and then pressure is quickly released, which causes the “explosion” of lignocellulosic biomass (Mood et al., 2013). Unlike steam explosion pretreatment, liquid hot water pretreatment uses high pressure to maintain water in 
liquid state, and solubilize hemicellulose into water consequently. It is believed that acetic acid and other acids are released from the lignocellulosic biomass, which in turn catalyze the cleavage of the linkages between lignin and hemicellulose to facilitate their solubilization (Mood et al., 2013).

\subsubsection{Chemical pretreatment}

\subsubsection{Acid pretreatment}

Acid pretreatment involves the use of concentrated or dilute acid to fractionate lignocellulosic biomass. Mineral acids are most commonly used, which include sulfuric acid $\left(\mathrm{H}_{2} \mathrm{SO}_{4}\right)$, phosphoric acid $\left(\mathrm{H}_{3} \mathrm{PO}_{4}\right)$, nitric acid $\left(\mathrm{HNO}_{3}\right)$, and hydrochloric acid $(\mathrm{HCl})$. Dilute acid pretreatment often results in significant solubilization of hemicellulose, but minor degradation of cellulose and lignin. Due to hemicellulose removal and subsequently exposed cellulose fibrils, pretreated biomass often gives enhanced digestibility. Moreover, solubilized hemicellulose could be further degraded into furfural and other byproducts under acidic conditions. Hemicellulose degradation products can react with each other during acid pretreatment, and polymerized into a lignin-like polymer, called pseudolignin (Sannigrahi et al., 2011). The formed pseudolignin can redeposit on cellulose and negatively affect the digestibility of cellulose by blocking the access of cellulase.

\subsubsection{Alkaline pretreatment}

Alkaline pretreatment mainly uses bases, such as sodium hydroxide, sodium carbonate, potassium hydroxide, calcium hydroxide, to selectively remove lignin and hemicellulose in lignocellulosic biomass. In alkaline pretreatment, ester and ether linkages bridging hemicelluloses and lignin are cleaved, which leads to the solubilization of these polymers in alkali solutions. Compared with hemicellulose, lignin is more prone to being broken down and solubilized during alkaline 
pretreatment. In addition to solubilizing lignin and hemicellulose, alkali pretreatment can lead to partial decrystallization of cellulose (Brodeur et al., 2011).

\subsubsection{Organic solvents}

Organic solvents pretreatment has a history of more than 100 years, and organic solvents used mainly include alcohols (e.g., ethanol, butanol, methanol), polyols (e.g., glycerol, ethylene glycol), carboxylic acids (e.g., formic acid, acetic acid), and ketones (e.g., acetone, dioxane) (Zhang et al., 2016b). In recent years, the use of renewable solvents, such as $\gamma$-valerolactone, formic acid, ethanol, have attracted much attention due to their overall sustainability. Such solvents are effective to fractionate lignocellulosic biomass into major components (i.e., cellulose, hemicellulose, lignin), which can be recovered and/or upgraded separately. However, recovering organic solvents after pretreatment is a costly process, which is a major limitation for organic solvent pretreatment.

\subsubsection{Ionic liquids (ILs)}

ILs are a group of salts in the form of liquids at low/room temperature, which is consisted of large organic cations and small inorganic anions. ILs feature low vapor pressure, thermal stability, and non-flammability. Depending on the types of anions and cations in ILs, ILs achieve efficient pretreatment performance via different mechanisms. For some ILs, the pretreatment mainly results in the dissolution of cellulose, which can be recovered/regenerated by adding anti-solvents. The recovered/regenerated cellulose often demonstrates decreased crystallinity, and thus enhanced digestibility (Dadi et al., 2006). For other ILs, the enhanced digestibility of lignocellulosic biomass mainly results from the dissolution of lignin and hemicellulose in ILs. Similar to organic solvent pretreatment, recovering ILs after 
pretreatment is necessary to reduce its cost, but energy-efficient recovery methods are still under investigation.

\subsubsection{Deep eutectic solvents (DESs)}

DES is a eutectic mixture composed of a hydrogen-bond acceptor (HBA) and a hydrogen-bond donor (HBD) (Abbott et al., 2004). The most commonly used HBA is quaternary ammonium salt (QAS), especially choline chloride $(\mathrm{ChCl})$, a biogenic and biodegradable chemical (Morandeira et al., 2017), while the HBDs can be a wide range of chemicals, including carboxylic acids and polyols. DESs are a related class of ILs and share attractive features of ILs. Compared to ILs, DESs are more ecologically friendlier and much cheaper, and possess more tunable physicochemical properties for different applications (Tang et al., 2017). DESs pretreatment is an emerging method for lignocellulosic biomass fractionation, and has attracted great attention in the recent 5 years (Satlewal et al., 2018). The DESs pretreatment can selectively solubilize lignin and hemicellulose, while keeping cellulose intact (Satlewal et al., 2018). Such high selectivity often results in cellulose-rich solid residues that can be facilely converted into glucose upon enzymatic hydrolysis.

\subsection{Limitations of current pretreatment}

As mentioned above, many pretreatments have been developed, with some successfully deployed at industrial scale. However, most pretreatments developed so far only aim to maximize cellulose conversion, while much less attention is paid to hemicellulose and lignin streams. It has been recognized that full utilization of all the three major components (i.e., cellulose, hemicellulose, lignin) with the least carbon loss could make the biorefinery more economically and ecologically competitive with petroleum refinery (Alonso et al., 2017). However, for lignin valorization into valueadded products, effective delignification and recovery of lignin with desired 
properties are critical. As is often the case, lignin is prone to depolymerization and repolymerization under different pretreatment conditions, which make producing tailor-made lignin very challenging. In addition, high solid loading enzymatic hydrolysis with high sugar yield is desired in modern biorefinery as it yields concentrated sugar hydrolysate. This allows the production of fuels and chemicals at high titer, which reduces the cost of downstream processing and waste management. Although decent sugar yields can be achieved via enzymatic hydrolysis with moderate solid loading, high solid loading enzymatic hydrolysis with satisfying yield remains a challenge for most pretreatments (Chen et al., 2016). In terms of hemicellulose, the liquor enriched with hemicellulose-derived $\mathrm{C}_{5}$ sugars can be further upgraded via either biological or catalytic routes to many valuable products. For biological upgrading, one major hurdle is the existence of many lignin- and carbohydratederived compounds, which inhibit microbial growth and cause low product yield and productivity (Palmqvist \& Hahn-Hägerdal, 2000). On the contrary, catalytic conversion is less affected by those inhibitors, and more atom-efficient as less carbon is utilized for cellular biomass synthesis and $\mathrm{CO}_{2}$ production. In short, to make the biorefinery more profitable and economically viable, there is still a need to develop novel processes to enable the production of lignin with desirable properties, cellulose pulp with good digestibility at high solid loading enzymatic hydrolysis, and efficient upgrading of hemicellulose streams into chemicals with high yield and carbon efficiency.

\subsection{Uniqueness of using QAS based solvents}

\subsubsection{High delignification and production of valorizable lignin}

Numerous DESs composed of QAS (e.g., ChCl) and biomass-derived carboxylic acids, such as formic acid (FA), lactic acid (LA), and oxalic acid (OA), 
have shown good delignification during pretreatment. The highest delignification of 98.5\% was reported by Zhang and co-workers with corn cob as feedstock and $\mathrm{ChCl}$ :OA as the pretreatment solvent (Zhang et al., 2016a). ChCl:LA also showed good performance in removing lignin from woody biomass and grass, with the highest delignification of $93.1 \%$ reported (Zhang et al., 2016a). Microwave-assisted pretreatment based on DESs, such as $\mathrm{ChCl}: \mathrm{LA}$ and $\mathrm{ChCl}: \mathrm{OA}$, can effectively extract more than 70\% lignin in several minutes (Chen \& Wan, 2018; Liu et al., 2017). Lignin-derived phenolic compounds, such as $p$-coumaric acid (PCA), have also been used as HBD. With ChCl:PCA as pretreatment solvent, delignification of $60.8 \%$ was achieved in a recent study under $160{ }^{\circ} \mathrm{C}$ for $3 \mathrm{~h}$ (Kim et al., 2018).

Polyol-based DESs consisted of $\mathrm{ChCl}$, and biomass-derived polyols (e.g., glycerol, ethylene glycol, and propylene glycol) are also called natural DESs (NADESs). Among those NADESs, ChCl:Glycerol (ChCl:Gly) is the one most studied as glycerol is a low-cost by-product produced from biodiesel industry. Decent lignin removal ranging from 60 to $90 \%$ were reported by using $\mathrm{ChCl}$ :Gly. For example, 59\% lignin removal from corn cob was reported by Procentese and his colleagues using ChCl:Gly (Procentese et al., 2015). With corn stover as feedstock, Xu et al. (Xu et al., 2018) reported similar lignin removal by conducting the pretreatment at $180{ }^{\circ} \mathrm{C}$ for $2 \mathrm{~h}$. More recently, Xia et al. (Xia et al., 2018) reported another efficient $\mathrm{ChCl}$ :Gly based pretreatment by "acidifying" it with a Lewis acid such as $\mathrm{AlCl}_{3}$, which showed near complete lignin dissolution at $120{ }^{\circ} \mathrm{C}$ for $4 \mathrm{~h}$.

DESs synthesized from $\mathrm{ChCl}$ and Urea, imidazole, and amine were also effective for solubilizing lignin during pretreatment. A common feature of such DESs is its basicity, which facilitates the selective solubilization of lignin from lignocellulosic biomass due to deprotonation of phenolic hydroxyl groups in lignin. 
Wheat straw pretreated by $\mathrm{ChCl}$ : monoethanolamine (ChCl:MEA) and $\mathrm{ChCl}$ : diethanolamine (ChCl:DEA) for $12 \mathrm{~h}$ at $90{ }^{\circ} \mathrm{C}$ demonstrated lignin removal of $81 \%$ and $73.5 \%$, respectively (Zhao et al., 2018). ChCl:imidazole could give similar lignin removal when it was used for pretreating corncob at $115^{\circ} \mathrm{C}$ for $15 \mathrm{~h}$ (Procentese et al., 2015).

Lignin property is a critical factor in determining lignin valorization potential and routes. Many studies have demonstrated that lignin recovered from DESs pretreatment own some unique properties beneficial to its valorization. Alvarez-Vasco et al. (Alvarez-Vasco et al., 2016) reported that lignin extracted from poplar using $\mathrm{ChCl}$ :LA demonstrated a low molecular weight, uncondensed structure, and a high purity of $95 \%$. Similarly, via microwave assisted $\mathrm{ChCl}$ :OA pretreatment, Liu et al. reported a low molecular weight and high-purity lignin from poplar (Liu et al., 2017). In addition, the recovered lignin also experienced an appreciable degree of demethylation, which increased its reactivity toward chemical modification (Liu et al., 2017). Such lignin could find their application in polymer synthesis due to their high purity and good reactivity. Recently, Chen et al. (Chen \& Wan, 2018) reported $\mathrm{ChCl}$ :Gly pretreatment of switchgrass yielded lignin with ether bonds preserved. Such lignin could be a good substrate for aromatic monomers production via catalytic depolymerization.

\subsubsection{Significantly enhanced cellulose digestibility}

In addition to effective delignification and production of lignin with desirable properties for valorization, the pretreatment using $\mathrm{ChCl}$ based DESs also yields cellulose pulp with good digestibility. Procentese et al. (Procentese et al., 2015) reported a glucose yield of $92 \%$ from corn cob pretreated by either ChCl:Gly or ChCl:imidazole. Using the same feedstock and DESs, Zhang et al. reported a glucose 
yield of $96.4 \%$ (Zhang et al., 2016a). Near theoretical glucose yield (99\%) was obtained from corn stover pretreated by ChCl:FA (Xu et al., 2016). Rice straw after sequential pretreatment using $\mathrm{ChCl}: \mathrm{OA}$ and $\mathrm{ChCl}: \mathrm{Urea}$ could give a glucose yield of 90\% (Hou et al., 2017). In contrast, pretreatment using other DESs, such as ChCl:PCA and ChCl:LA, led to decreased, but still decent glucose yields ( $80 \%$ ) (Kim et al., 2018; Zhang et al., 2016a).

\subsubsection{Platform chemical production}

Furfural (FF) and 5-hydroxymethylfurfural (5-HMF) are two important platform chemicals that can be upgraded into advanced fuels, chemicals, and materials (Kucherov et al., 2018; Li et al., 2016). Both platform chemicals can be produced from lignocellulosic biomass and its derived carbohydrates (i.e. xylose,

glucose, fructose). The use of $\mathrm{ChCl}$-based solvents as catalytic systems for converting carbohydrate into furans is very promising because high yield of products can be obtained at mild conditions.

A furfural yield of $60 \%$ was achieved with hemicellulose-derived xylose as an substrate and $\mathrm{ChCl}$ :LA as a reaction medium at $100{ }^{\circ} \mathrm{C}$ for $60 \mathrm{~min}$ (Zhang et al., 2014). A similar furfural yield was obtained using $\mathrm{ChCl}$ :citric acid (ChCl:CA) as a reaction medium (Zhang \& Yu, 2013). In terms of HMF production, fructose can be effectively converted to HMF by forming a DES with $\mathrm{ChCl}$, and a yield as high as $90.3 \%$ was obtained at $100{ }^{\circ} \mathrm{C}$ for $4 \mathrm{~h}$ using with $\mathrm{HCl}$ as a catalyst (Zuo et al., 2017). An even higher HMF yield of $94.2 \%$ was reported by the same group using the same solvent system, but with amberlyst-15 as the catalyst (Zuo et al., 2018). In addition to HMF production from cellulose-derived sugars, HMF-derived chemicals (e.g., levulinic acid) can also be directly produced from lignocellulosic biomass-derived carbohydrates using ChCl-based solvent system. For example, Murat et al. reported a 
levulinic acid yield of $76.2 \%$ from cellulose in only 1 minute at $180{ }^{\circ} \mathrm{C}$ under microwave heating with $\mathrm{ChCl}: \mathrm{OA}$ as the reaction medium (Sert et al., 2018). Using aqueous $\mathrm{ChCl}$ as solvent and $\mathrm{AlCl}_{3}$ as catalyst, Bayu et al. reported a levulinic acid yield of $51.7 \%$ from glucose under $155^{\circ} \mathrm{C}$ in $90 \mathrm{~min}$ (Bayu et al., 2016).

ChCl-based solvents appear to not only enhance polysaccharide conversion into renewable chemicals via biological routes by efficiently fractionating it into different streams, but also directly convert polysaccharide into platform chemicals in a single step. Its versatility provides new opportunities to upgrade lignocellulosic biomass into a wide spectrum of products.

\subsection{Scopes of this work}

Given the advantages of QAS-based green solvents discussed above, this work is focused on the development of novel processes for biomass pretreatment and conversion into renewable chemicals using QAS-based green solvents. Although lignin with properties desired for upgrading can be obtained with different $\mathrm{ChCl}$ based solvents, there is a lack of platform solvent systems capable of tuning lignin properties during pretreatment. Thus, in Chapter 2, a $\mathrm{ChCl}$ :EG based platform solvent system was developed for effectively fractionating lignocellulosic biomass and production of tailor-made lignin. Built on the results from Chapter 2, the feasibility of using $\mathrm{ChCl}$ :EG and a series of ChCl-based ternary DESs for pretreatment at high solid loading was explored in Chapter 3 and 4. Chapter 5 reports the effectiveness of aqueous $\mathrm{ChCl}$ as a novel solvent for pretreatment, and its effects on cellulose digestibility, lignin properties, and xylose conversion to furfural. Stemming from effective biomass pretreatment using aqueous $\mathrm{ChCl}$, a one-pot process using a biphasic aqueous $\mathrm{ChCl}$ / methyl isobutyl ketone (MIBK) solvent system was developed for simultaneous biomass fractionation and furfural production and the 
results were reported in Chapter 6. Lastly, Chapter 7 concludes the work and suggests future studies. 


\section{Chapter 2 A Tunable Platform Deep Eutectic Solvent for the Integrated Biorefinery}

\subsection{Introduction}

Lignocellulosic biomass is a renewable resource for chemical and fuel production. It is comprised of three major components, namely cellulose, hemicellulose, and lignin. Due to its inherent recalcitrance, a pretreatment step is often necessary to enable efficient polysaccharide conversion via biological valorization routes. In the past decades, researchers have devoted to developing different pretreatment methods to improve sugar production from lignocellulosic biomass, but often paid less attention to lignin recovery and further upgrading (Kumar et al., 2009; Sun et al., 2016b). Not until recently, lignin valorization gains increasing interest for enabling an economically viable biorefinery (Alonso et al., 2017; Ragauskas et al., 2014b).

Different technical routes, such as catalytic and biological conversions, have been proposed to upgrade lignin into a wide range of products (e.g., aromatics, lipids, antioxidants) (He et al., 2017; Lauberts et al., 2017; Shuai et al., 2016a). However, the selection of lignin valorization route largely depends on lignin structures and properties. For example, it has been shown that a higher percentage of $\beta$-O-4 linkage in lignin, a higher yield of aromatic monomers depolymerized from lignin. (Phongpreecha et al., 2017; Renders et al., 2017) In the case of using lignin for polyurethane production, high purity is one of the most desirable attributes (Lora \& Glasser, 2002). In terms of lignin-based carbon fiber production, high homogeneity, high molecular weight, and more condensed structure could contribute to desired mechanical properties of carbon fiber (He et al., 2017; Hosseinaei et al., 2016). In a 
conventional bio-refinery prioritizing cellulose digestibility, lignin is often generated in poor quality and with untailored properties, leading to low valorization potential.

The concept of "lignin-first" biorefinery has thus been proposed, concerning lignin extraction with desirable properties while ensuring cellulose digestibility upon biomass processing (Huang et al., 2018). However, it would be challenging to generate both digestible cellulose and valorizable lignin in a single pretreatment. Dilute acid pretreatment is such an example, which yields cellulose pulp with good digestibility, but lignin condensation occurring during the pretreatment limits its valorization potential (Moxley et al., 2012). Active stabilization of ether bonds in lignin via formaldehyde during pretreatment was reported to prevent lignin condensation, which led to near theoretical monomer yields via catalytic depolymerization, but the digestibility of cellulose pulp was not enhanced (Shuai et al., 2016a). Therefore, it is of particular importance for "lignin-first" biorefinery to develop pretreatment processes capable of tailoring lignin properties for different applications while improving cellulose digestibility.

Deep eutectic solvents (DESs) are designer solvents featuring low cost, renewability, low vapor pressure, and biodegradability (Smith et al., 2014; Zhang et al., 2012). Owing to such attractive properties, DESs have been widely used as solvents and reaction media in many applications (Bubalo et al., 2015; Chen \& Wan, 2018; Cui et al., 2015). Some DESs, such as choline chloride:ethylene glycol (ChCl:EG ) and $\mathrm{ChCl}$ :formic acid ( $\mathrm{ChCl}: \mathrm{FA})$, show great potential in biomass fractionation. Such DESs are capable of solubilizing lignin and hemicellulose as well as improving cellulose digestibility (Xu et al., 2016; Zhang et al., 2016a). ChCl:EG is a promising DES for lignocellulosic biomass processing as it is compatible with cellulase and microorganisms, has excellent performance in processing lignocellulosic 
biomass at high solid loading, and can be facilely recovered via scalable electrodialysis (Chen et al., 2018a; Gunny et al., 2015; Liang et al., 2019). Notwithstanding these appealing properties of $\mathrm{ChCl}$ :EG, its potential for tuning the lignin properties during pretreatment remains to be explored.

In addition to lignin and cellulose streams, upgrading of hemicellulose streams is also of great importance to improve the overall economics of biorefinery. The hemicellulose streams can be used for furfural production via catalytic dehydration of xylose or for lipid and carotenoid production via biological conversion (Chen et al., 2018a; Chen \& Wan, 2018). Furfural as a platform chemical can be used as precursors for biofuel and biopolymers, and has an increasing market demand (Mariscal et al., 2016). Previous studies also demonstrated that DESs are good reaction media for furfural production as they can accelerate the furfural production rate (Zhang et al., 2014). To enable high yield furfural production, the extraction of furfural into another inert organic phase is preferred by preventing its rapid degradation. Ideally, the extraction phase should be a solvent that is environmentally benign, safe to store, and has a low boiling point and cost. Biomass derived aprotic solvents (e.g., THF, MIBK, $\gamma$-valerolactone) are appealing solvents for furfural extraction due to their renewability and good capability to solubilize furfural. Among these solvents, MIBK is the most commonly used one as it has a relatively low boiling point compared with $\gamma$-valerolactone, and dose not form explosive peroxide during storage as compared to THF (Alonso et al., 2013; Saha \& Abu-Omar, 2014). Industrially, MIBK is synthesized from acetone via aldol condensation, dehydration, and hydrogenation. As the precursor for synthesizing MIBK, acetone is also a biomass derived aprotic solvent, but has a much lower boiling point and cost. It seemed to be more reasonable and economical to use acetone as an extraction phase for furfural production. 
However, acetone is rarely used as the extraction solvent because it is highly miscible with most aqueous solutions. Salting-out is a method used for separating water miscible acetone from aqueous solution (Xie et al., 2015). Given that the ChCl:EG has a high content of salt (i.e., $\mathrm{ChCl}$ ), it is possible that acetone could form a biphasic system with neat/aqueous $\mathrm{ChCl:EG}$. The application of this system would enable facile production and recovery of furfural from $\mathrm{ChCl}$ :EG liquor with solubilized hemicellulose from the pretreatment.

In this study, we explored the potential of $\mathrm{ChCl}$ :EG for lignocellulosisc biomass processing. In an attempt to tune the lignin properties during $\mathrm{ChCl}$ :EG based switchgrass pretreatment, effects of pretreatment temperature, acid concentration, water content, and $\mathrm{ChCl}$ to $\mathrm{EG}$ ratio were investigated. The lignin properties were characterized and compared using 2D HSQC NMR. The digestibility of cellulose pulp obtained at different conditions was also evaluated by conducting enzymatic hydrolysis at $20 \mathrm{wt} \%$ solid loading. Lastly, the feasibility of using aqueous $\mathrm{ChCl}$ :EG/acetone biphasic system for furfural production from hemicellulose stream was tested.

2.2 Materials and methods

\subsubsection{Feedstocks}

Switchgrass was collected from the South Farm at University of Missouri in Columbia, Missouri, USA. It was air-dried, ground through $2 \mathrm{~mm}$ screen, and stored in a plastic bag. Hydrolytic enzymes (Cellic ${ }^{\circledR}$ CTec2 and HTec2) were kindly provided by Novozyme (Franklinton, NC, USA). All the chemicals were purchased from Fisher Scientific (Hampton, NH, USA). 


\subsubsection{DES Synthesis, pretreatment and lignin recovery}

$\mathrm{ChCl}$ was dried at $80^{\circ} \mathrm{C}$ for $6 \mathrm{~h}$ and cooled to room temperature in a desiccator prior to use. $\mathrm{ChCl}$ :EG was synthesized by heating the mixture of $\mathrm{ChCl}$ and EG with continuous stirring $(200 \mathrm{rpm})$ until a transparent solvent was formed. The pretreatment conditions and solvents tested were listed in Table 1. In a typical run, $5 \mathrm{~g}$ switchgrass was mixed with $45 \mathrm{~g}$ acidified $\mathrm{ChCl}: \mathrm{EG}$ in a $250 \mathrm{~mL}$ glass bottle reactor. Then, the bottle was heated in a preheated oil bath for 30 min with stirring at $200 \mathrm{rpm}$. After pretreatment, the bottle was taken out from the oil bath and cooled to below 50 ${ }^{\circ} \mathrm{C}$, followed by adding $100 \mathrm{~mL}$ acetone-water mixture $(1 / 1, \mathrm{v} / \mathrm{v})$ to the pretreatment slurry. After vacuum filtration, the solid residue was washed with $50 \mathrm{~mL}$ acetonewater mixture twice, and then stored at $-20^{\circ} \mathrm{C}$ for further use. The filtrate collected from slurry separation and solid washing was combined and acetone was removed under reduced pressure. Lignin precipitated out upon acetone removal was collected via centrifugation at $12,000 \times \mathrm{g}$ for $5 \mathrm{~min}$, further washed with deionized (DI) water for four times, and then dried at $45^{\circ} \mathrm{C}$ for $12 \mathrm{~h}$. The dried lignin was stored in a desiccator at room temperature prior to further use. The liquor after lignin precipitation was collected and stored at $-20{ }^{\circ} \mathrm{C}$ before further use.

\subsubsection{Enzymatic hydrolysis}

Enzymatic hydrolysis was conducted with $20 \%(\mathrm{w} / \mathrm{w})$ solid loading in citrate buffer (50 mM, pH 5.5) for $48 \mathrm{~h}$. CTec2 and HTec2 were added at 20 and $2 \mathrm{mg}$ protein/g solid, respectively. The hydrolysis was conducted in an incubator shaker with the temperature of $50{ }^{\circ} \mathrm{C}$ and the agitation rate of $150 \mathrm{rpm}$. After enzymatic hydrolysis, the slurry was diluted 10 times to determine the sugar yield as described by Jan B Kristensen et al (Kristensen et al., 2009). 


\subsubsection{Furfural production}

Furfural production was performed in an optimized $9 \mathrm{~mL}$ solvent system containing $2 \mathrm{~mL}$ pretreatment liquor, and $7 \mathrm{ml}$ acetone at $180{ }^{\circ} \mathrm{C}$ for $30 \mathrm{~min}$ in a pressure tube (Ace Glass, Vineland, NJ). The processes for optimizing furfural production were as follows. Partition coefficient for furfural in acetone/aqueous ChCl:EG (1:2) biphasic system was determined by stirring the biphasic system containing $2 \mathrm{ml}$ aqueous $\mathrm{ChCl}: \mathrm{EG}(1: 2), 5 \mathrm{ml}$ acetone, and $140 \mathrm{mg}$ furfural for $30 \mathrm{~min}$ at $800 \mathrm{rpm}$. After settling for $10 \mathrm{~min}$, the volumes of aqueous phase and acetone phase were measured, and the furfural concentration in each phase was determined with high performance liquid chromatography (HPLC). Kinetics studies were performed at $180^{\circ} \mathrm{C}$ in a pressure tube containing $2 \mathrm{~mL}$ aqueous $\mathrm{ChCl:EG} \mathrm{(1:2)} \mathrm{and}$

$5 \mathrm{~mL}$ acetone. The aqueous phase containing $50 \mathrm{~g} / \mathrm{L}$ xylose, $1.0 \% \mathrm{H}_{2} \mathrm{SO}_{4}$, and $2 \mathrm{wt} \%$ $\mathrm{AlCl}_{3}$. The volumetric ratio of aqueous $\mathrm{ChCl}$ :EG (1:2) to acetone was optimized by fixing the aqueous volume at $2 \mathrm{~mL}$, while varying the acetone volume from 5 to 8 $\mathrm{mL}$. The catalyst $\left(\mathrm{AlCl}_{3}\right)$ loading was optimized by varying the catalyst loading from $1.0 \%(\mathrm{w} / \mathrm{v})$ to $2.0 \%(\mathrm{w} / \mathrm{v})$. The liquor obtained from pretreatment conducted at $130{ }^{\circ} \mathrm{C}$ for 30 min using $\mathrm{ChCl}: \mathrm{EG}(1: 2)$ containing $1.0 \% \mathrm{H}_{2} \mathrm{SO}_{4}$ was used as a model liquor, which contained 30\% water and $16.35 \mathrm{~g} / \mathrm{L}$ xylose (oligomers and monomers).

\subsubsection{Analytical methods}

Compositional analysis. The composition of the biomass samples and lignin purity were determined following NREL laboratory protocol (Sluiter et al., 2008). Concentration of sugar monomers and furfural was determined using HPLC (Agilent 1200). 
HSQC NMR. The 2D HSQC NMR spectra was acquired on a Bruker AVIII $800 \mathrm{MHz}$ spectrometer using Bruker supplied pulse sequence named hsqcetgp at 50 ${ }^{\circ} \mathrm{C}$ throughout the NMR acquisition. The spectral width is $15.4 \mathrm{ppm}$ for ${ }^{1} \mathrm{H}$ and 170.0 ppm for ${ }^{13} \mathrm{C}$. A total of $2048\left({ }^{1} \mathrm{H}\right)$ complex points were acquired with $256\left({ }^{13} \mathrm{C}\right)$ time increments. The ${ }^{1} \mathrm{~J}_{\mathrm{CH}}$ was set to $145 \mathrm{~Hz}$ which is the average one-bond $\mathrm{C}-\mathrm{H}$ coupling constant. The number of scans was 48 and the repetition delay was $1.5 \mathrm{~s}$. The ${ }^{13} \mathrm{C}$ dimension was zero-filled to 2048 points before the data was subjected to a sinesquared window function (sifted $90^{\circ}$ ) and Fourier Transformation. The chemical shift axes were calibrated with respect to the solvent signals $(2.49 \mathrm{ppm}$ for residual proton and 39.5 ppm for ${ }^{13} \mathrm{C}$ ).

\subsection{Results and discussion}

\subsubsection{Characterization of pretreated switchgrass}

$\mathrm{ChCl}$ :EG has been reported as a good DES for solubilizing kraft lignin (Di Marino et al., 2017), but when used for biomass pretreatment, it only solubilized approximately $25 \%$ lignin from rice straw after $6 \mathrm{~h}$ of pretreatment at $120^{\circ} \mathrm{C}$ (Hou et al., 2018). In another study, $\mathrm{ChCl}$ :EG was shown to solubilize near $90 \%$ lignin from corn cob, but required $24 \mathrm{~h}$ at a relatively modest temperature $\left(90^{\circ} \mathrm{C}\right)$ (Zhang et al., 2016a). Our previous study has shown that acidification of DESs composed of $\mathrm{ChCl}$ and polyols (e.g., glycerol, EG) can significantly improve delignification and hemicellulose removal (Chen \& Wan, 2018). In this study, we further explored the performance of acidified $\mathrm{ChCl}: \mathrm{EG}$ in lignocellulosic biomass fractionation.

Figure 2.1 and 2.2 depict the compositional change of switchgrass in response to $\mathrm{ChCl}$ :EG pretreatment under varying conditions. We first tested the effects of acid concentration and temperature on the compositional change. $\mathrm{ChCl}: \mathrm{EG}(1: 2)$ removed more than $70 \%$ lignin and xylan from switchgrass under an acidic environment $(0.5 \%$ 
$\mathrm{H}_{2} \mathrm{SO}_{4}$ ), which only took 30 min at $110{ }^{\circ} \mathrm{C}$. With $0.5 \% \mathrm{H}_{2} \mathrm{SO}_{4}$ concentration, increasing the temperature from 110 to $150{ }^{\circ} \mathrm{C}$ led to an increase in lignin removal from $74 \%$ to $85 \%$ and an increase in xylan removal from $71 \%$ to $80 \%$. A similar trend was observed with $1.0 \% \mathrm{H}_{2} \mathrm{SO}_{4}$ concentration. On the other hand, when the temperature was kept same, a higher acid concentration led to more xylan and lignin removal. Especially at a lower temperature $\left(110^{\circ} \mathrm{C}\right)$, acid had more effects on lignin removal. In contrast to lignin removal, acid increase led to similar increases in xylan removal $(9 \sim 11 \%)$ across the three temperatures.

Water can tune physicochemical properties of DESs (e.g., viscosity, basicity), and improve the performance of DESs during biomass processing (Kumar et al., 2016a; Soares et al., 2017). We thus investigated the effects of water addition on the pretreatment performance by adding $20 \%$ water to $\mathrm{ChCl}: \mathrm{EG}(1: 2)$. When the temperature was $110{ }^{\circ} \mathrm{C}$, aqueous $\mathrm{ChCl}: \mathrm{EG}(1: 2)$ gave $77.7 \%$ and $72.3 \%$ xylan and lignin removal, which were 2.4 and $10 \%$ lower than its counterpart with no water added (Figure 2.2). By increasing the temperature to $130{ }^{\circ} \mathrm{C}$, the switchgrass pretreated by aqueous $\mathrm{ChCl}$ :EG (1:2) demonstrated a slightly lower lignin removal than that from neat $\mathrm{ChCl}: \mathrm{EG}(1: 2)$ pretreatment, but 7\% higher xylan removal (Figure 2.2). On one hand, the addition of water reduced the viscosity and facilitated mass transfer, which, in turn, would enhance the dissolution of xylan in DES (Kumar et al., 2016a). On the other hand, water is not a good solvent for lignin dissolution as the lignin-water interactions are not energetically favorable (Smith et al., 2016). It has been reported that the strong hydrogen bond interactions between EG and free hydroxyl groups present in lignin enhance lignin dissolution (Sun et al., 2016a). Water could be deemed as a special "HBD", which can interact with DESs via hydrogen bonding interaction (Durand et al., 2013). The presence of water may 
weaken the interaction between $\mathrm{ChCl}: \mathrm{EG}$ and lignin, and less lignin was dissolved in the solvent system consequently.

Lastly, we investigated the effects of increasing molar ratio of $\mathrm{HBA}$ (i.e., $\mathrm{ChCl}$ ) to $\mathrm{HBD}$ (i.e. $\mathrm{EG}$ ) on the pretreatment performance. Since $\mathrm{ChCl}$ :EG (3:2) was not able to maintain a stable liquid state at room temperature, $20 \%$ water was added to it before pretreatment. The lignin and xylan removal by $80 \% \mathrm{ChCl}: \mathrm{EG}(3: 2)$ pretreatment showed no difference to those by $80 \% \mathrm{ChCl}$ :EG $(1: 2)$ pretreatment using the same conditions $\left(1.0 \% \mathrm{H}_{2} \mathrm{SO}_{4}, 110{ }^{\circ} \mathrm{C}, 30 \mathrm{~min}\right)$ (Figure 2.1$)$, but were still about $10 \%$ lower than those by $100 \% \mathrm{ChCl}$ :EG (1:2). Reducing the water content from $20 \%$ to $10 \%$ in aqueous $\mathrm{ChCl}$ :EG (3:2) resulted in a 5\% higher xylan removal and $3.5 \%$ higher lignin removal (Figure 2.2). Consistent with results using 100\% ChCl:EG for pretreatment, when acid concentration was decreased from $1.0 \%$ to $0.5 \%$ in $80 \%$ and 90\% ChCl:EG (3:2), less xylan and lignin were removed (Figure 2.2). However, it was interesting to note that using same pretreatment conditions $\left(1.0 \% \mathrm{H}_{2} \mathrm{SO}_{4}, 110{ }^{\circ} \mathrm{C}\right.$, $30 \mathrm{~min}$ ), 90\% ChCl:EG (3:2) gave a 6\% higher xylan removal, but 9\% less lignin removal than $100 \% \mathrm{ChCl}: \mathrm{EG}(1: 2)$. A similar trend was observed with pretreatment with $80 \% \mathrm{ChCl}$ :EG (1:2) and 100\% ChCl:EG (1:2) containing $1.0 \% \mathrm{H}_{2} \mathrm{SO}_{4}$ under $130{ }^{\circ} \mathrm{C}$ for $20 \mathrm{~min}$ (Figure 2.2). Thus, by tuning the HBA to HBD ratio and water content in DESs, it is possible to tune the selectivity of xylan and lignin dissolution in $\mathrm{ChCl}: \mathrm{EG}$, and therefore, their content in cellulose pulp. These cellulose pulp may find their application in the cellulose based functional materials requiring different amount of lignin to tailor their properties (Bian et al., 2017).

Under all the conditions tested, more than 90\% cellulose was preserved (Figure 2.2), indicating that acidified $\mathrm{ChCl}$ :EG selectively solubilizing lignin and hemicellulose. Due to significant removal of lignin and hemicellulose during 
pretreatment, the recovered solid residue showed an enriched cellulose content ranging from $60 \%$ to $80 \%$ (Figure 2.1 ).

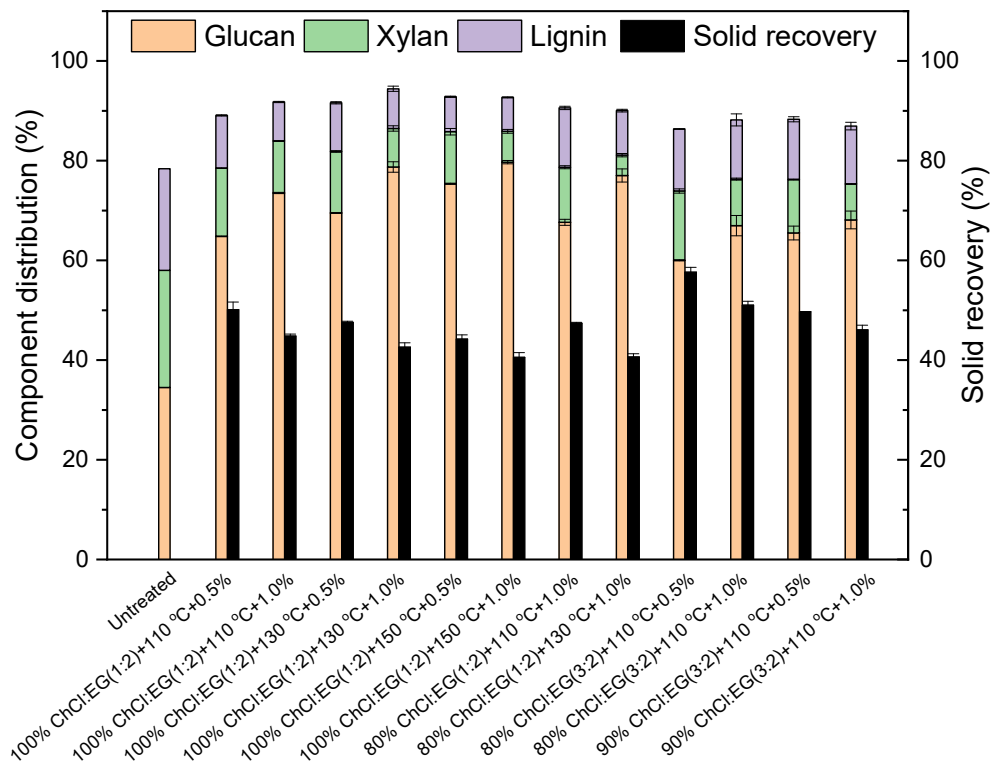

Figure 2.1 Switchgrass component distribution and solid recovery upon pretreatment.

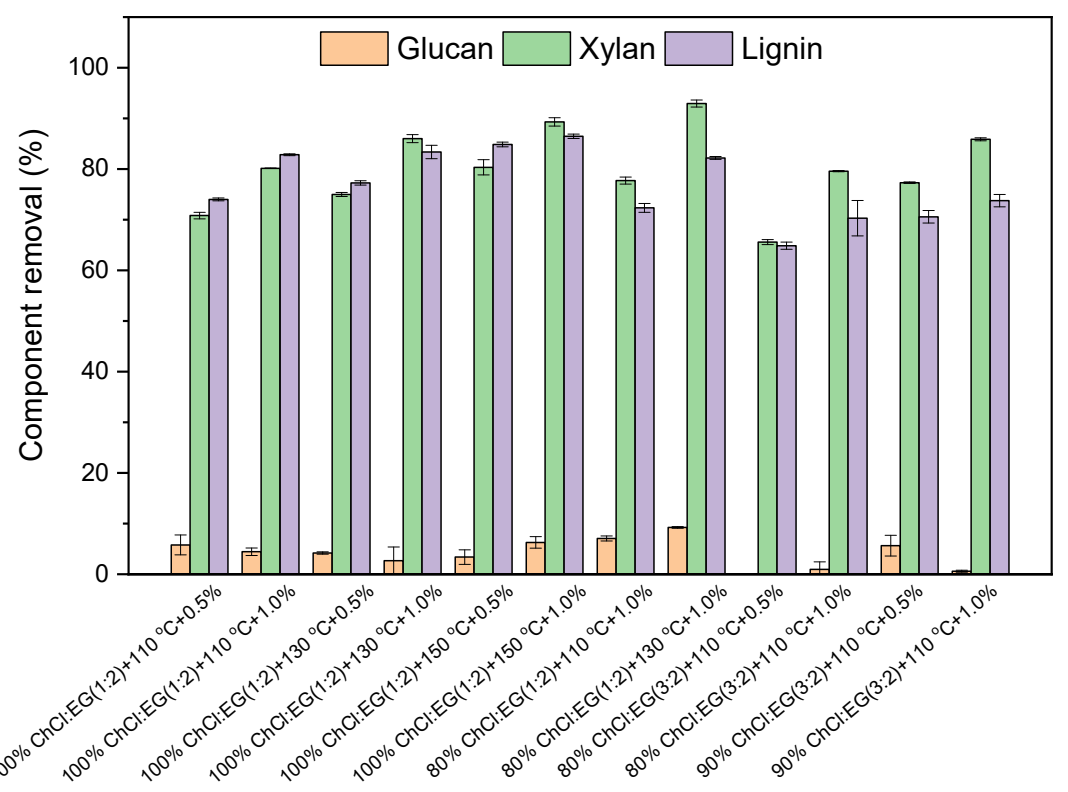

Figure 2.2 Switchgrass component removal upon pretreatment. 


\subsubsection{Sugar production via high solid loading enzymatic hydrolysis}

From economical and energy-saving perspectives, high solid loading enzymatic hydrolysis is desired as it results in concentrated sugar hydrolysate, which facilitates its further use for fermentation and catalytic upgrading (Geng et al., 2015). Therefore, we investigated sugar production of pretreated switchgrass at $20 \%(\mathrm{w} / \mathrm{w})$ high solid loading enzymatic hydrolysis. As shown in Figure 2.3 and 2.4, for switchgrass pretreated using 100\% ChCl:EG (1:2) under all the tested conditions, the glucose yields of $80 \%$ or above and xylose yields of $70 \%$ or above were achieved after $48 \mathrm{~h}$ of enzymatic hydrolysis. When the pretreatment was conducted with a lower acid concentration $(0.5 \%)$, temperature increase had no or minor effects on sugar yields. Nonetheless, the glucose concentration increased from $134 \mathrm{~g} / \mathrm{L}$ to $159 \mathrm{~g} / \mathrm{L}$ and the corresponding total monomeric sugar concentration increased from $159 \mathrm{~g} / \mathrm{L}$ to 179 $\mathrm{g} / \mathrm{L}$. This increase was attributed to a higher enriched cellulose content in switchgrass from pretreatment at higher temperature due to more xylan and lignin removal (Figure 2.1). Temperature effects on sugar yields became significant when the acid concentration was increased to $1 \%$. As a result of temperature increase from 110 to $130{ }^{\circ} \mathrm{C}$ when the acid concentration was $1 \%$, the glucose yield increased from $87 \%$ to $90 \%$ and the xylose yield from $81 \%$ to $94 \%$ (Figure 2.3 ). Correspondingly, the glucose concentration in the hydrolysate increased from 162 to $182 \mathrm{~g} / \mathrm{L}$ (Figure 2.4). It should be noted that further increasing the temperature from 130 to $150{ }^{\circ} \mathrm{C}$ led to no increase in sugar yields and concentrations.

Under the same pretreatment conditions $\left(1.0 \% \mathrm{H}_{2} \mathrm{SO}_{4}, 130{ }^{\circ} \mathrm{C}, 30 \mathrm{~min}\right)$, the acidic aqueous $\mathrm{ChCl}$ :EG (1:2) containing 20\% water showed a lower glucose yield than the neat one, while the xylose yield was not affected and remained above $90 \%$ (Figure 2.3 ). Such differences could stem from the different modification of 
switchgrass during pretreatment by different solvents. As shown in Figure 2.5, the switchgrass pretreated by $100 \% \mathrm{ChCl}: \mathrm{EG}(1: 2)$ under $130{ }^{\circ} \mathrm{C}$ presented a grey color, while the one pretreated with aqueous $\mathrm{ChCl}$ :EG (1:2) showed a brown color, suggesting that possible deposition of lignin and/or pseudolignin onto the cellulose surface. As discussed above, water, as a "bad" solvent, can cause the aggregation of lignin and/or pseudolignin, which can deposit on the cellulose. Such deposited lignin could block the access of enzymes to cellulose via non-productive binding of enzymes (Sannigrahi et al., 2011; Smith et al., 2016). In addition, the adsorption of enzymes to lignin can also cause the denaturation of enzymes on lignin surfaces, and in turn, decreases the sugar yield (Börjesson et al., 2007). It has been suggested that the phenolic hydroxyl groups in lignin contributed significantly to cellulase inactivation, and modification of phenolic hydroxyl groups helps alleviate the enzyme inaction (Sewalt et al., 1997). Under acidic conditions, EG could react with the phenolic hydroxyl groups in lignin, and resulted in a decrease of total phenolic groups in lignin (Jasiukaitytė et al., 2010). The presence of water can slow down the reaction between lignin and EG, and therefore, left the lignin in the cellulose pulp less modified (Moghaddam et al., 2014). As a result, the switchgrass pretreated by aqueous ChCl:EG (1:2) demonstrated a lower glucose yield. Not surprisingly, the glucose yield for the switchgrass pretreated by $80 \% \mathrm{ChCl}$ :EG (1:2) was lower than the one pretreated using neat $\mathrm{ChCl}$ :EG (1:2) under same conditions $\left(1.0 \% \mathrm{H}_{2} \mathrm{SO}_{4}, 110{ }^{\circ} \mathrm{C}, 30\right.$ min). Similarly, using the same pretreatment conditions, the switchgrass pretreated by 80\% $\mathrm{ChCl}$ :EG (3:2) with a higher $\mathrm{ChCl}$ to $\mathrm{EG}$ ratio demonstrated a lower digestibility. However, reducing the water content from $20 \%$ to $10 \%$ in aqueous $\mathrm{ChCl}: \mathrm{EG}(3: 2)$ did improve the digestibility as both the glucose yield and xylose yield reached near $80 \%$ (Figure 2.3). Similar glucose yield was obtained for the one 
pretreated by $90 \% \mathrm{ChCl}: \mathrm{EG}(3: 2)$ containing $0.5 \% \mathrm{H}_{2} \mathrm{SO}_{4}$ under $110{ }^{\circ} \mathrm{C}$ for $30 \mathrm{~min}$. Correspondingly, the total monomeric sugars in the hydrolysate reached $151 \mathrm{~g} / \mathrm{L}$ for the one pretreated with $\mathrm{ChCl}: \mathrm{EG}(3: 2)$ containing $0.5 \% \mathrm{H}_{2} \mathrm{SO}_{4}$, and $174 \mathrm{~g} / \mathrm{L}$ for the other one with $1.0 \% \mathrm{H}_{2} \mathrm{SO}_{4}$, respectively (Figure 2.4 ).

Overall, among all the conditions tested, the pretreatment conducted using $100 \%$ ChCl:EG (1:2) under $130{ }^{\circ} \mathrm{C}$ and $1.0 \% \mathrm{H}_{2} \mathrm{SO}_{4}$ led to the highest glucose and xylose yields, both reaching over $90 \%$, as well as the highest total monomeric sugar concentration of $203 \mathrm{~g} / \mathrm{L}$ in the hydrolysate. Our results indicated that switchgrass pretreated by acidified $\mathrm{ChCl}: \mathrm{EG}$ under a relatively mild condition was well suited to high solid enzymatic hydrolysis. The resultant hydrolysate with high sugar concentration can be readily used for fermentative production of high-titer biofuels and chemicals.

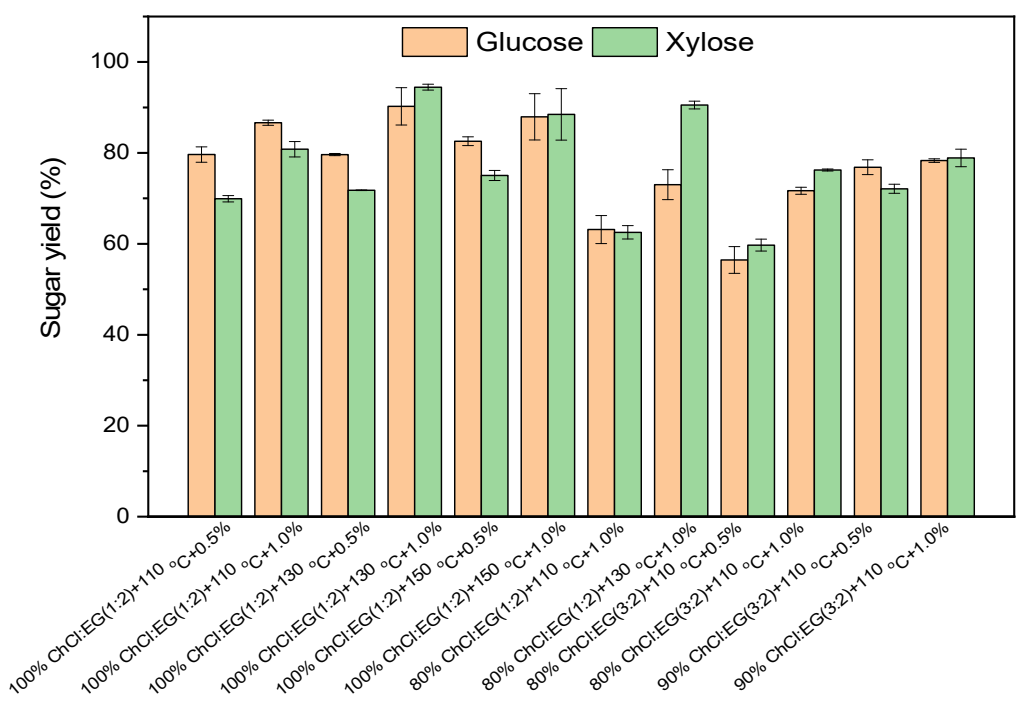

Figure 2.3 Digestibility of pretreated switchgrass upon pretreatment. 


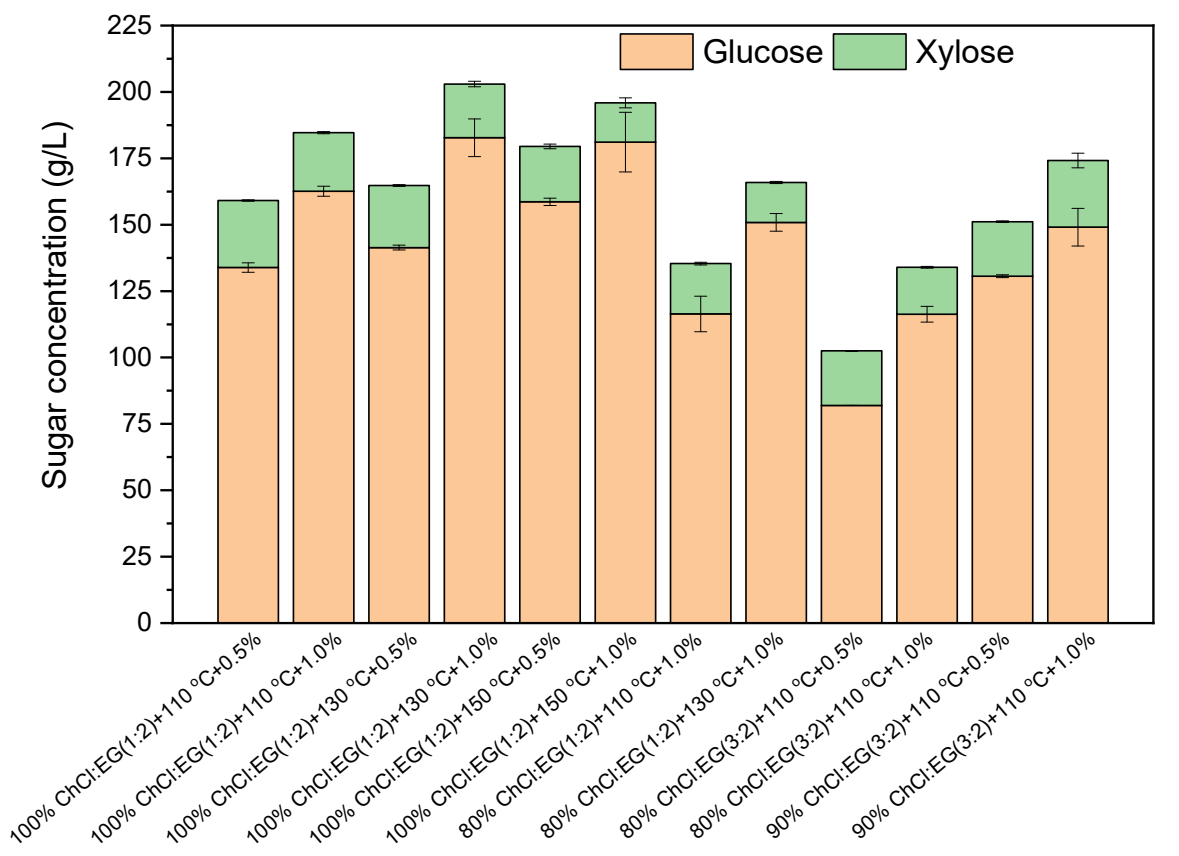

Figure 2.4 Monomeric sugar concentration in the hydrolysates.

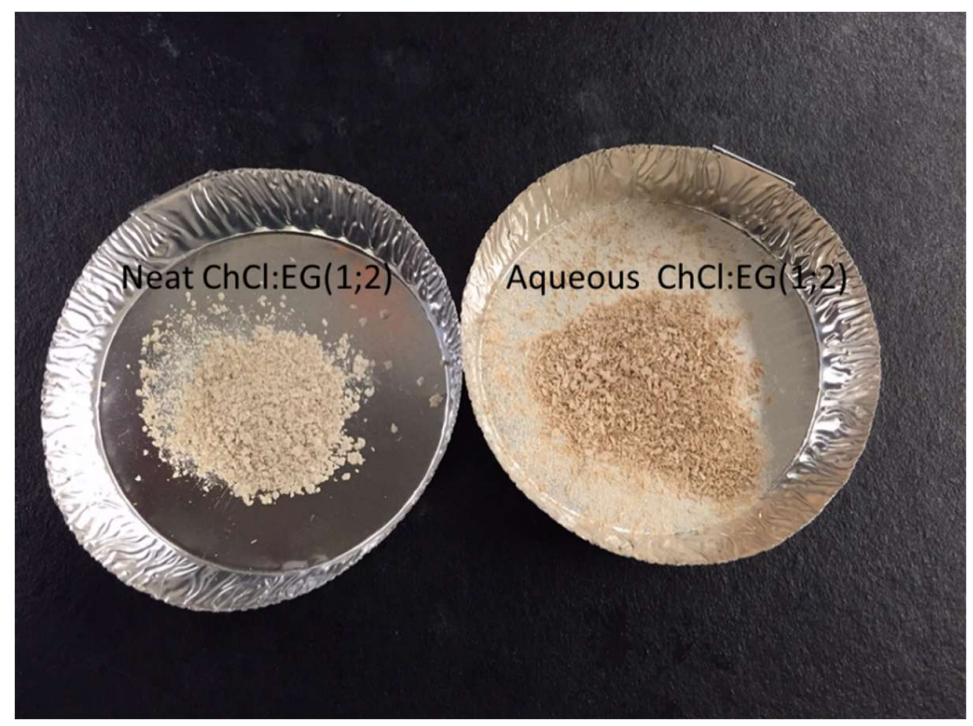

Figure 2.5 Pretreated switchgrass using acidified $\left(1 \% \mathrm{H}_{2} \mathrm{SO}_{4}\right)$ neat $\mathrm{ChCl}$ :EG(1:2) (left) and aqueous $\mathrm{ChCl}: \mathrm{EG}(1: 2)$ (right) under $130^{\circ} \mathrm{C}$ for $30 \mathrm{~min}$. 


\subsubsection{Lignin properties}

\subsubsection{Lignin purity}

Purity is an important property for lignin, and lignin with high purity can find its potential application in many fields, such as the production of polyurethane foams, adhesives, and epoxies (Lauberts et al., 2017). As shown in Table 2.1, for the pretreatment using neat $\mathrm{ChCl}$ :EG (1:2), lignin purity increased from $79 \%$ to $87 \%$ upon temperature increase from 110 to $150{ }^{\circ} \mathrm{C}$ when acid was loaded at $0.5 \%$. This increased purity could be partly attributed to the more carbohydrate removal from lignin under higher temperature (Table 2.1). With a higher acid (1.0\%), lignin purity showed a similar increasing trend upon temperature increase from 110 to $130{ }^{\circ} \mathrm{C}$, but further increasing temperature to $150{ }^{\circ} \mathrm{C}$ did not increase the purity. When the pretreatment temperature was kept at 110 or $130^{\circ} \mathrm{C}$, increasing acid concentration from $0.5 \%$ to $1 \%$ led to an increase in the purity of recovered lignin due in part to a higher removal of carbohydrate with a higher acid concentration (Table 2.1). However, the effects of acid increase on lignin purity diminished at a higher temperature $\left(150^{\circ} \mathrm{C}\right)$, and a similar carbohydrate content was shown for both lignin (L5\&L6). Interestingly, it was found that the recovered lignin from $100 \% \mathrm{ChCl}$ :EG (1:2) pretreatment under all conditions contains an appreciable amount of EG (Table 2.1). As mentioned above, EG can react with many functional groups in depolymerized lignin. For example, EG can react with the carbonyl groups on depolymerized lignin to form acetal (Figure 2.6\&2.7). Although such reactions introduced "impurities" (i.e., EG) in lignin, it may also reduce the recondensation reaction of depolymerized lignin (Figure 2.7).

Water addition to $\mathrm{ChCl}: \mathrm{EG}(1: 2)$ reduced both the $\mathrm{EG}$ and carbohydrate contents in the recovered lignin. As a result, the lignin (L7\&8) from pretreatment under $110{ }^{\circ} \mathrm{C}$ 
and $130{ }^{\circ} \mathrm{C}$ using aqueous $\mathrm{ChCl}$ :EG (1:2) showed a purity of 86.46 and $91.36 \%$, which were near 3\% higher than those from pretreatment using $100 \% \mathrm{ChCl}: \mathrm{EG}(1: 2)$ (L2\&6) (Table 2.1). As explained above, adding water may deaccelerate the reaction between EG and lignin, but facilitate better carbohydrate solubilization. Similarly, the lignin (L9-12) recovered from ChCl:EG (3:2) pretreatment showed smaller amounts of carbohydrate and EG than the lignin recovered from $100 \% \mathrm{ChCl}: \mathrm{EG}(1: 2)$ pretreatment under same conditions (Table 2.1). 
Table 2.1 Composition and purity of lignin

\begin{tabular}{llclll}
\hline Lignin code & Solvent ${ }^{\mathrm{a}}$ & $\begin{array}{c}\text { Reaction Conditions }\left({ }^{\circ} \mathrm{C},\right. \\
\left.\mathrm{wt} \% \mathrm{H}_{2} \mathrm{SO}_{4}\right)\end{array}$ & $\begin{array}{l}\text { Carbohydrates in } \\
\text { lignin }(\%)\end{array}$ & $\begin{array}{l}\text { EG in lignin } \\
(\%)\end{array}$ & $\begin{array}{l}\text { Lignin purity } \\
(\%)\end{array}$ \\
\hline L1 & $100 \%$ ChCl:EG (1:2) & $110,0.5$ & $4.11 \pm 0.45$ & $7.17 \pm 0.25$ & $78.66 \pm 1.23$ \\
L2 & $100 \%$ ChCl:EG (1:2) & $110,1.0$ & $1.06 \pm 0.17$ & $7.12 \pm 0.19$ & $82.94 \pm 0.71$ \\
L3 & $100 \%$ ChCl:EG (1:2) & $130,0.5$ & $2.22 \pm 0.03$ & $6.98 \pm 0.30$ & $84.62 \pm 0.10$ \\
L4 & $100 \%$ ChCl:EG (1:2) & $130,1.0$ & $1.13 \pm 0.34$ & $6.58 \pm 0.14$ & $88.35 \pm 0.22$ \\
L5 & $100 \%$ ChCl:EG (1:2) & $150,0.5$ & $1.29 \pm 0.09$ & $7.54 \pm 0.19$ & $86.90 \pm 0.31$ \\
L6 & $100 \%$ ChCl:EG (1:2) & $150,1.0$ & $1.42 \pm 0.37$ & $6.76 \pm 0.14$ & $87.22 \pm 0.02$ \\
L7 & $80 \%$ ChCl:EG (1:2) & $110,1.0$ & $0.66 \pm 0.00$ & $5.76 \pm 0.04$ & $86.46 \pm 0.84$ \\
L8 & $80 \%$ ChCl:EG (1:2) & $130,1.0$ & $0.36 \pm 0.00$ & $4.34 \pm 0.11$ & $91.36 \pm 0.25$ \\
L9 & $80 \%$ ChCl:EG (3:2) & $110,0.5$ & $2.61 \pm 0.25$ & $2.98 \pm 0.04$ & $81.52 \pm 2.78$ \\
L10 & $80 \%$ ChCl:EG (3:2) & $110,1.0$ & $0.99 \pm 0.04$ & $3.07 \pm 0.00$ & $87.02 \pm 1.79$ \\
L11 & $90 \%$ ChCl:EG (3:2) & $110,0.5$ & $0.99 \pm 0.07$ & $3.67 \pm 0.01$ & $86.93 \pm 0.40$ \\
L12 & $90 \%$ ChCl:EG (3:2) & $110,1.0$ & $0.44 \pm 0.01$ & $3.60 \pm 0.09$ & $90.42 \pm 0.31$ \\
\hline
\end{tabular}

${ }^{a}$ The ratios in parenthesis stand for the molar ratios of $\mathrm{ChCl}$ to EG. The percentages stand for the contents of CC-EG in the pretreatment solvents. Differing from $\mathrm{ChClEG}(1: 2), \mathrm{ChCl}: \mathrm{EG}(3: 2)$ is in the solid form at room temperature.

${ }^{\mathrm{b}}$ Percentages of the impurities as carbohydrates or EG in the recovered raw DES lignin. 


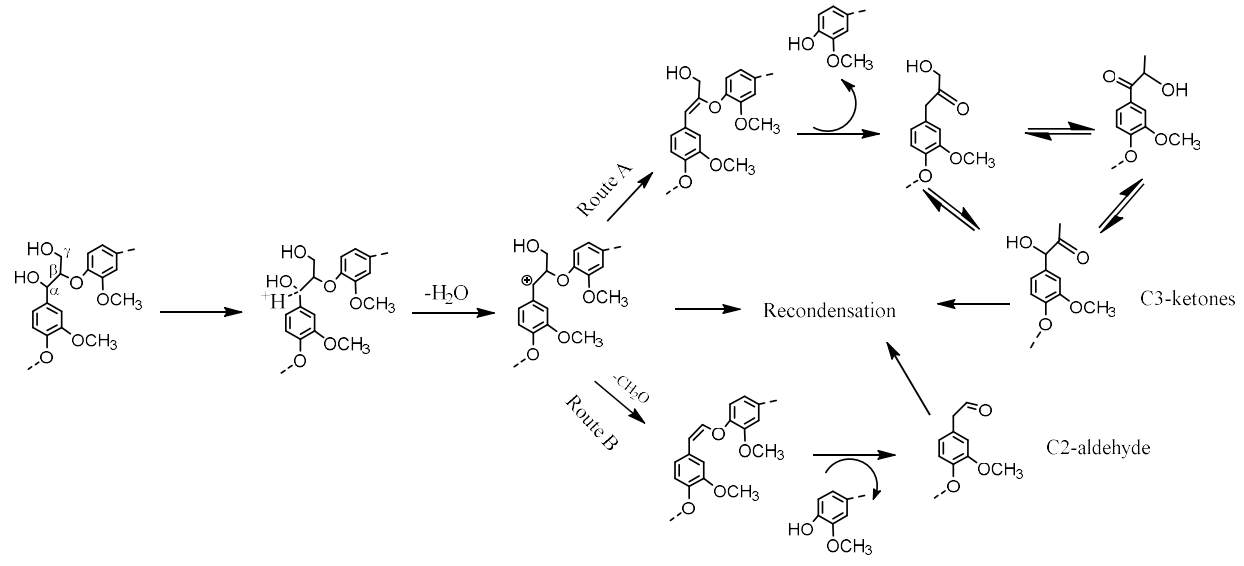

Figure 2.6 Illustration of acid catalyzed cleavage of $\beta-O-4$ linkage in lignin.

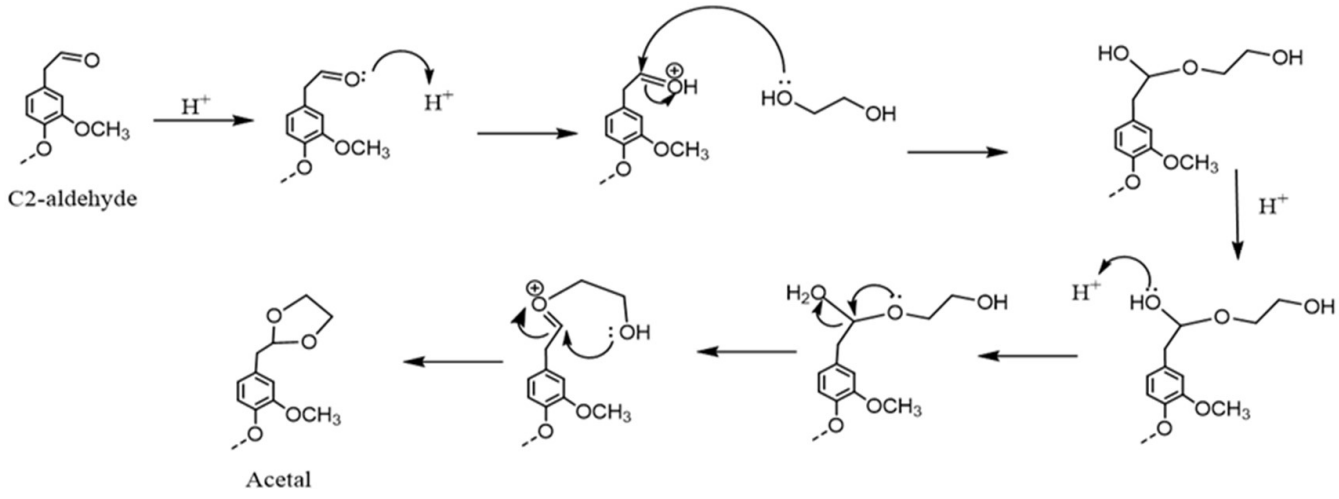

Figure 2.7 Proposed reaction of EG with depolymerized lignin fraction under acidic condition. 


\subsubsection{2D HSQC NMR analysis of lignin}

To understand chemical transformation of lignin in response to pretreatment conditions, native lignin (NL) and lignin extracted under different conditions were examined using 2D HSQC NMR. Main cross-signals in the HSQC spectra of switchgrass lignin were assigned according to prior studies (Figure 2.8) (Samuel et al., 2011; Samuel et al., 2010). For NL, the $\beta$-O-4 unit (A) was the major peak shown in the aliphatic regions, while $\beta-5(B)$ and $\beta-\beta(C)$ units were present at the smaller contents. Compared with NL, lignin recovered from the pretreatment using $100 \%$ $\mathrm{ChCl}$ :EG (1:2) under different conditions demonstrated a varying extent of the cleavage of $\beta$-O-4 linkages. Lignin obtained under milder conditions (e.g., L1) showed more preserved $\beta-\mathrm{O}-4$ linkages than lignin obtained from severer conditions (e.g., L2) (Figure 2.8\&Table 2.2). When the temperature was increased to $130{ }^{\circ} \mathrm{C}$ or above, the complete cleavage of $\beta-\mathrm{O}-4$ linkages in lignin (L3 to L6) was shown despite the acid concentration used in the pretreatment. These results were in accordance with those from other studies which showed that the cleavage of $\beta-\mathrm{O}-4$ linkages was more significant under severer conditions (Chen et al., 2017; Gschwend et al., 2018).

When 20\% water was added to $\mathrm{ChCl}$ :EG (1:2), the recovered lignin (L7 to L8) showed a more native lignin-like structure and a higher percentage of $\beta-O-4$ linkages than the one from neat $\mathrm{ChCl}: \mathrm{EG}(1: 2)$ pretreatment under the same conditions $\left(110{ }^{\circ} \mathrm{C}, 1.0 \% \mathrm{H}_{2} \mathrm{SO}_{4}\right)$ (Figure 2.8\&Table 2.2). As illustrated in Figure 2.6, the acidcatalyzed cleavage of $\beta$-O-4 linkages is initiated by protonation of the $\alpha$-position, followed by dehydration and breaking of the ether bonds. In aqueous $\mathrm{ChCl}$ : $\mathrm{EG}$, the stabilization of proton by water led to decreased availability of the acidic protons, which in turn slowed down the reactions involved in the cleavage of $\beta-\mathrm{O}-4$ linkages 
(Mellmer et al., 2014). Eventually, more ether bonds were preserved in lignin (L7) extracted using aqueous $\mathrm{ChCl}: \mathrm{EG}(1: 2)$ under a milder condition (i.e., $110{ }^{\circ} \mathrm{C}, 1.0 \%$ $\mathrm{H}_{2} \mathrm{SO}_{4}$ ). However, when the temperature was elevated to $130^{\circ} \mathrm{C}$, no ether bonds were observed (L8), suggesting that increasing temperature promoted proton activity and ether bond cleavage. This was also consistent with what was observed with neat ChCl:EG (1:2) based pretreatment as discussed above.

Interestingly, it was found that when the molar ratio of $\mathrm{ChCl}$ to $\mathrm{EG}$ was increased from 1:2 to 3:2, the recovered lignin (L10) demonstrated a much higher abundance of $\beta$-O-4 linkages than $\mathrm{L} 7$ under the same pretreatment conditions $\left(110^{\circ} \mathrm{C}, 30 \mathrm{~min}, 1.0 \%\right.$ $\mathrm{H}_{2} \mathrm{SO}_{4}$ ) (Figure2.8\&Table 2.2). More strikingly, unlike most other pretreatment (e.g., alkali pretreatment) (Sun et al., 2015), the preservation of more $\beta$-O-4 linkages was not at the expense of delignification. As shown in Figure 2.2, similar delignification (70\%) was reached by the pretreatment using either $80 \% \mathrm{ChCl}: \mathrm{EG}(3: 2)$ or $80 \%$ ChCl:EG (1:2). Decreasing the acid concentration to $0.5 \%$ in $80 \% \mathrm{ChCl}$ : $\mathrm{EG}(3: 2)$ resulted in a lignin (L9) with even higher abundance of $\beta$-O-4 linkages (Figure 2.8\&Table 2.2), while the delignification was only slightly decreased from $70 \%$ to $65 \%$. When the water content was reduced from $20 \%$ to $10 \%$ in aqueous $\mathrm{ChCl}: \mathrm{EG}$ (3:2) containing $0.5 \% \mathrm{H}_{2} \mathrm{SO}_{4}$, the abundance of $\beta-\mathrm{O}-4$ linkages was decreased in $\mathrm{L} 11$ (Figure2.8 \&Table 2.2), but was still higher than that of either L1 or L7. It appeared that a higher $\mathrm{ChCl}$ content was beneficial to the preservation $\beta-\mathrm{O}-4$ linkages. Prior study revealed that halide anions (e.g., $\mathrm{Cl}^{-}, \mathrm{Br}^{-}$) can stabilize the carboncation formed (Enslow \& Bell, 2015). Plausibly, one mechanism for better preservation of $\beta-\mathrm{O}-4$ linkages during pretreatment with a higher molar ratio of $\mathrm{ChCl}$ to $\mathrm{EG}$ is the enhanced stabilization of $\beta-\mathrm{O}-4$ linkages with the increased $\mathrm{Cl}^{-}$concentration (Figure 2.9). Further study is needed to understand and validate the stabilization mechanisms of $\beta$ - 
O-4 linkages in $\mathrm{ChCl}$ :EG with different molar ratio of $\mathrm{HBA}$ to HBD. In addition, the delignification of $70 \%$ was also very close to those from pretreatments using other solvents, such as methanol and $\gamma$-valerolactone, which yield lignin with wellpreserved $\beta$-O-4 linkages (Luo \& Abu-Omar, 2018; Luterbacher et al., 2015). The cleavage of $\beta-\mathrm{O}-4$ linkages led to the formation of reactive intermediates, which undergoes the coupling to form $\mathrm{C}-\mathrm{C}$ bonds with higher breakdown energy (Figure 2.6).(Mittal et al., 2017b) The preservation of $\beta-O-4$ linkages is critical for the high yield production of aromatic monomers via lignin depolymerization (Phongpreecha et al., 2017). In addition, the digestibility of cellulose pulp was also not compromised with better retention of $\beta-\mathrm{O}-4$ linkages. For example, the switchgrass from pretreatment using 100\% $\mathrm{ChCl}$ : $\mathrm{EG}(1: 2)$ (corresponding to $\mathrm{L} 1)$ and $90 \% \mathrm{ChCl}(3: 2)$ (corresponding to L11) containing $0.5 \% \mathrm{H}_{2} \mathrm{SO}_{4}$ gave a similar glucose yield of $80 \%$ upon enzymatic hydrolysis at $20 \%(\mathrm{w} / \mathrm{w})$ solid loading (Figure 2.3$)$. In contrast, L11 had a 24\% higher $\beta-O-4$ linkages than L1 (Table 2.2).

In the aromatic region, the cross signals for syringyl (S), guaiacyl $(\mathrm{G})$, ferulate (FA) and $p$-coumarate ( $p$-CA) units are predominant in NL (Figure 2.8). Compared with NL, lignin (L1 to L6) recovered from neat $\mathrm{ChCl}$ :EG (1:2) pretreatment demonstrated a shrinkage of contours for these units (Figure 2.8). The reduced intensity for the peaks of S and G were was evident in L5 and L6 than in L1 and L2 (Figure 2.8). This was probably due to a more condensed structure formed in L5 and L6 under severer conditions as any condensation reaction occurring at 2-,5-,and 6positions could make this position lack of hydrogen and not be detectable by the HSQC NMR.(Chen et al., 2017) The FA units were still visible in L1, L2, and L3, but was absent in L4, L5, and L6 (Fig.2.8\&Table 2.2). In grass lignin, FA crosslinks lignin and hemicellulose via the formation of ether and ester linkages (Brandt et al., 
2015; Rodriguez et al., 2017). The disappearance of FA suggested the complete solubilization of FA in ChCl:EG under severer conditions (Dutta et al., 2018; Mittal et al., 2017b). In terms of the lignin recovered from aqueous $80 \% \mathrm{ChCl}$ :EG (1:2) pretreatment at $110{ }^{\circ} \mathrm{C}$ and $1.0 \% \mathrm{H}_{2} \mathrm{SO}_{4}(\mathrm{~L} 7)$, the peaks for $\mathrm{S}$ and $\mathrm{G}$ showed a higher intensity than those in L2. This is probably because in $\mathrm{L} 7$, the $\beta-\mathrm{O}-4$ interunits were less cleaved, which reduced the condensation reactions occurring at 2-, 5-, and 6positions in $\mathrm{S}$ and $\mathrm{G}$ units. Likewise, L9, L11, and L12 with a higher extent of $\beta-\mathrm{O}-4$ interunits also demonstrated a higher peak intensity for S and G units. 

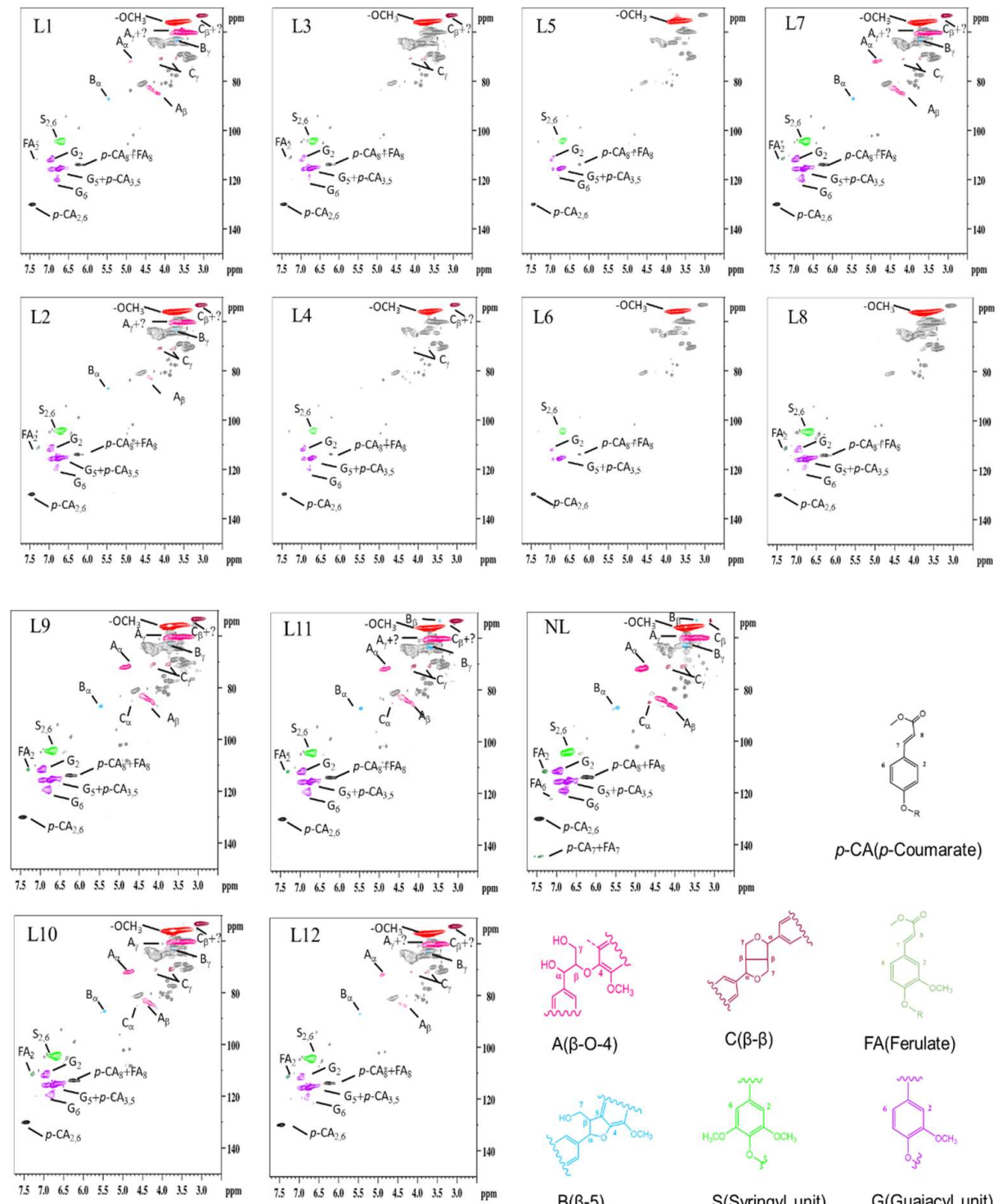

$p-\mathrm{CA}(p-$ Coumarate $)$

$B(\beta-5)$

S(Syringyl unit)

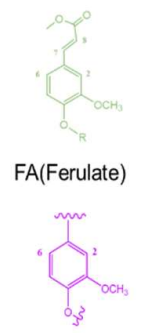

(Guaiacyl unit)

Figure 2.8 2D HSQC NMR spectra of lignin. L1-12 represent lignin samples listed in Table 2.1. 


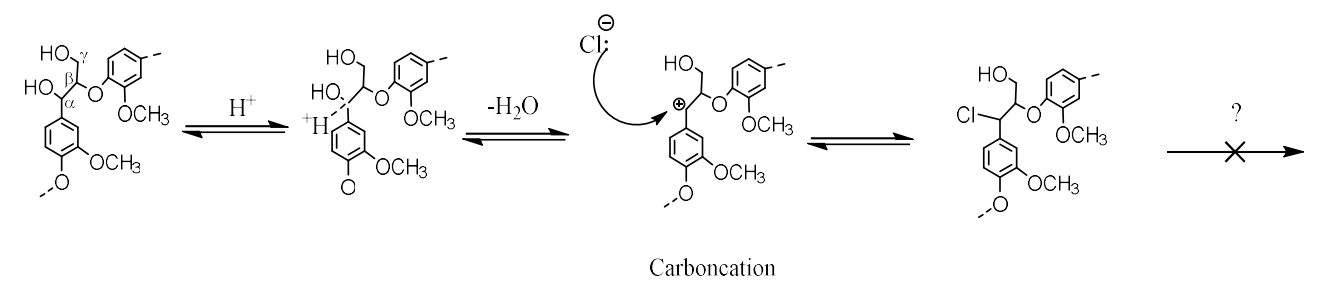

Figure 2.9 Stabilization of carbocation intermediate by $\mathrm{Cl}-1$ in acid catalyzed cleavage of $\beta-O-4$ linkage.

Table 2.2 Volume integration of $\beta-\mathrm{O}-4$ interlinkages and ferulate in lignin

\begin{tabular}{lll}
\hline Lignin & $\beta-\mathrm{O}-4$ & FA \\
\hline NL & 46.7 & 9.35 \\
L1 & 9.8 & 3.9 \\
L2 & 3.8 & 5.6 \\
L3 & $/$ & 4.5 \\
L4 & $/$ & $/$ \\
L5 & $/$ & $/$ \\
L6 & $/$ & $/$ \\
L7 & 24.4 & 5.8 \\
L8 & $/$ & 6.8 \\
L9 & 40.3 & 6.53 \\
L10 & 31.0 & 6.91 \\
L11 & 33.7 & 6.66 \\
L12 & 15.0 & 5.61 \\
\hline
\end{tabular}


2.4. Furfural production from pretreatment liquor

In an attempt to use acetone as extraction solvent for furfural production from pretreatment liquor containing xylan derived sugars (i.e., xylose, oligosaccharides), we first tested the miscibility between $\mathrm{ChCl}$ :EG and acetone. As shown in Fig. 2.10, acetone is immiscible with both neat $\mathrm{ChCl}: \mathrm{EG}(1: 2)$ and aqueous $\mathrm{ChCl}: \mathrm{EG}(1: 2)$ containing $20 \%$ water. However, compared with aqueous $\mathrm{ChCl:EG(1:2)/acetone}$ biphasic system, the $\mathrm{ChCl}$ :EG/acetone one was less stable and crystallization of $\mathrm{ChCl}$ was observed. We also evaluated whether $\mathrm{ChCl}$ :carboxylic acid (e.g., formic acid, acetic acid, lactic acid) DESs can form stable biphasic systems with acetone.

$\mathrm{ChCl}$ :formic acid is miscible with acetone and can only form a mono-phase, while the $\mathrm{ChCl}$ :acetic acid led to the immediate crystallization of $\mathrm{ChCl}$. Only ChCl:LA can form a stable two phases with acetone, but it alone is not effective for lignocellulosic biomass fractionation as reported from previous studies (Kumar et al., 2016a). Thus, among the above DESs, only ChCl:EG demonstrated both good performance in biomass fractionation and feasibility of forming bi-phases with acetone. Given that aqueous ChCl:EG (1:2) can form a more stable bi-phase with acetone than neat $\mathrm{ChCl}: \mathrm{EG}(1: 2)$, we only focused on the performance of aqueous $\mathrm{ChCl}: \mathrm{EG}(1: 2)$ in furfural production in latter tests. We then investigated if furfural is preferably partitioned into the acetone phase, which is one criteria for a good furfural extraction solvent. As demonstrated in Figure 2.11, the partition coefficient for aqueous ChCl:EG(1:2) containing $10 \%$ water was about 5 when the volumetric ratio of acetone to aqueous $\mathrm{ChCl}: \mathrm{EG}(1: 2)$ was 2.5 . Increasing water content to $20 \%$ and $30 \%$ only resulted in a slightly lower partition coefficient (Fig. 2.11). Correspondingly, about $92 \%$ of the furfural was partitioned into the acetone phase under the tested 
conditions, and therefore, can be easily recovered by removing the low-boiling point acetone.

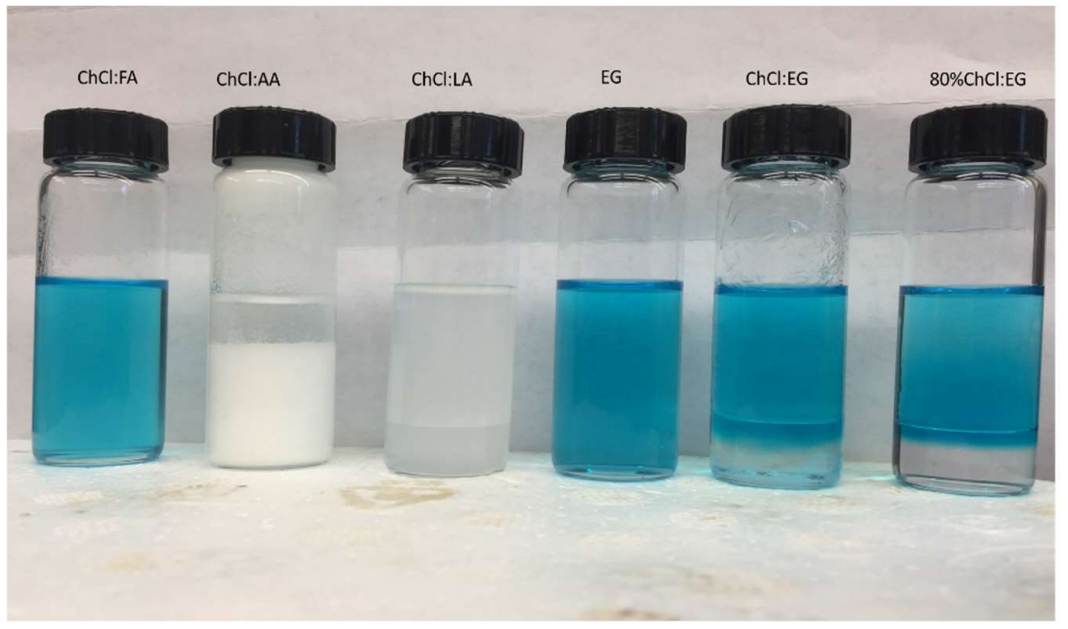

Figure 2.10 Phase formation between different DESs and acetone.

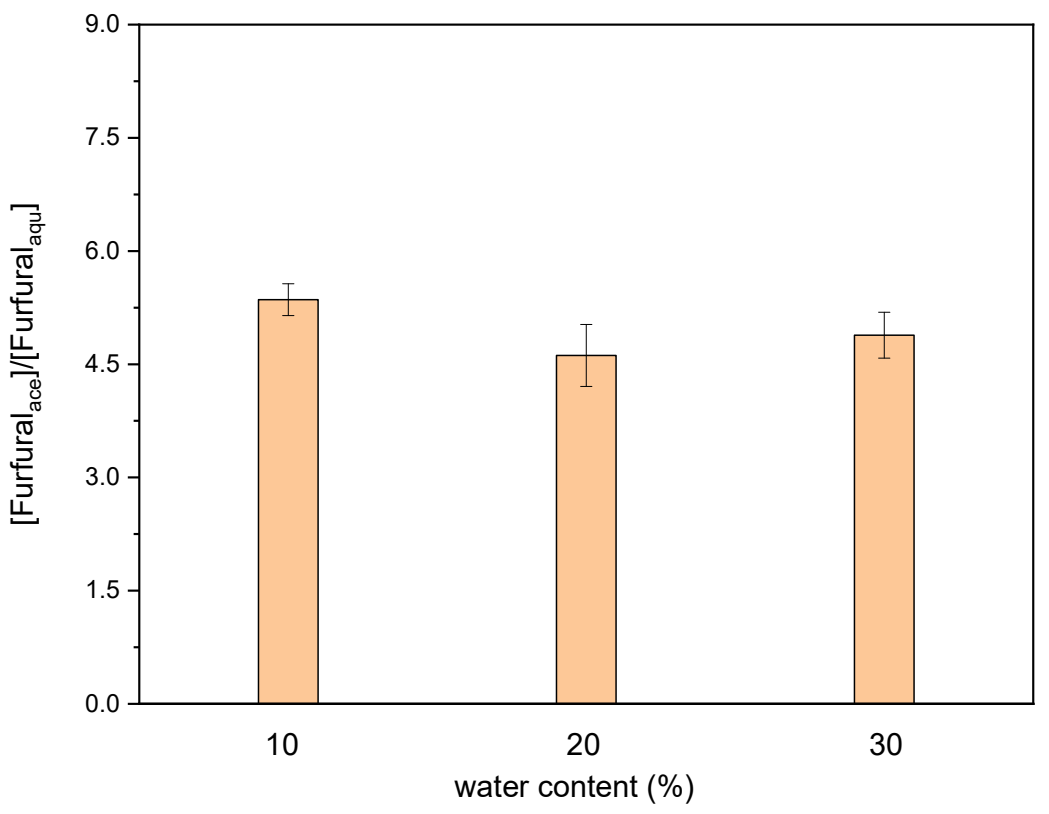

Figure 2.11 Partition coefficient for furfural in aqueous $\mathrm{ChCl}: \mathrm{EG}(1: 2) /$ Acetone biphasic system. 
Based on the above inspiring results for furfural partition, we further explored the furfural production from xylose in the aqueous $\mathrm{ChCl}$ :EG (1:2)/acetone biphasic system. As shown in Fig. 2.12, the furfural yields for acetone/aqueous ChCl:EG (1:2) containing $10 \%$ and $20 \%$ water systems were plautaued at $15 \mathrm{~min}$, reaching $59 \%$ and $65 \%$, respectively, while that for acetone/aqueous $\mathrm{ChCl}$ :EG (1:2) with $30 \%$ water peaked at $30 \mathrm{~min}$, reaching $72 \%$. It appeared that adding water was beneficial to the furfural production as the yield increased with a higher water content. This is probably due to the less side reactions in a water richer solvents, such as the acetalization between EG and furfural. Provided that aqueous CC-EG (1:2) with 30\% water gave the highest furfural yield at 30 min reaction, it was used for the following tests. Increasing the volumetric ratio from 5:2 to $6: 2$ and 7:2 resulted in an increase of furfural yield from 70.9 to $72.5 \%$ and $75.6 \%$, respectively (Figure 2.13 ). Further increasing the volume ratio did not improve the furfural yield, but instead, a lower yield was obtained. Lastly, the catalyst $\left(\mathrm{AlCl}_{3}\right)$ loading was optimized by testing three loadings: $1.0,1.5$ and $2.0 \mathrm{w} / \mathrm{v} \%$. It was found that a catalyst loading of $1.5 \mathrm{w} / \mathrm{v} \%$ gave the highest furfural yield of $76.5 \%$ (Fig. 2.14). Therefore, the optimized conditions for furfural production was as follows: $180{ }^{\circ} \mathrm{C}, 30 \mathrm{~min}, 1.5 \mathrm{w} / \mathrm{v} \% \mathrm{AlCl}_{3}$, and an volumetric ratio of 7:2 of the acetone to aqueous ChCl:EG (1:2, 30\% water). 


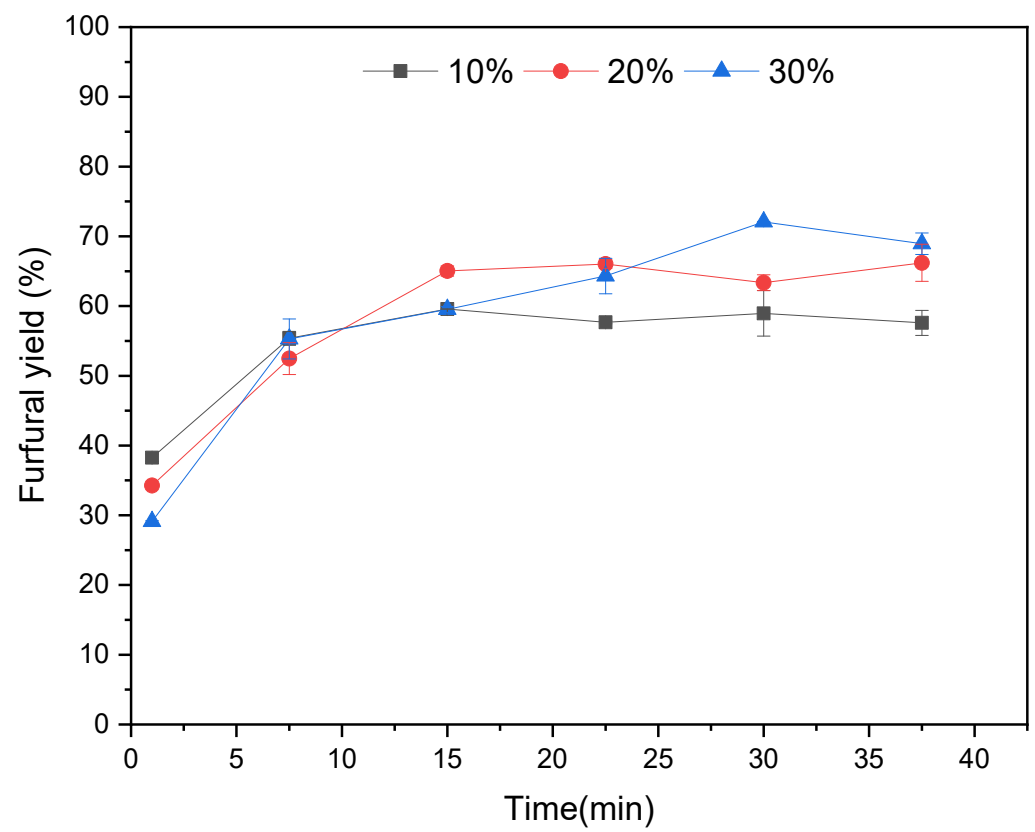

Figure 2.12 Kinetics of furfural production in aqueous $\mathrm{ChCl}$ :EG/Acetone biphasic systems.

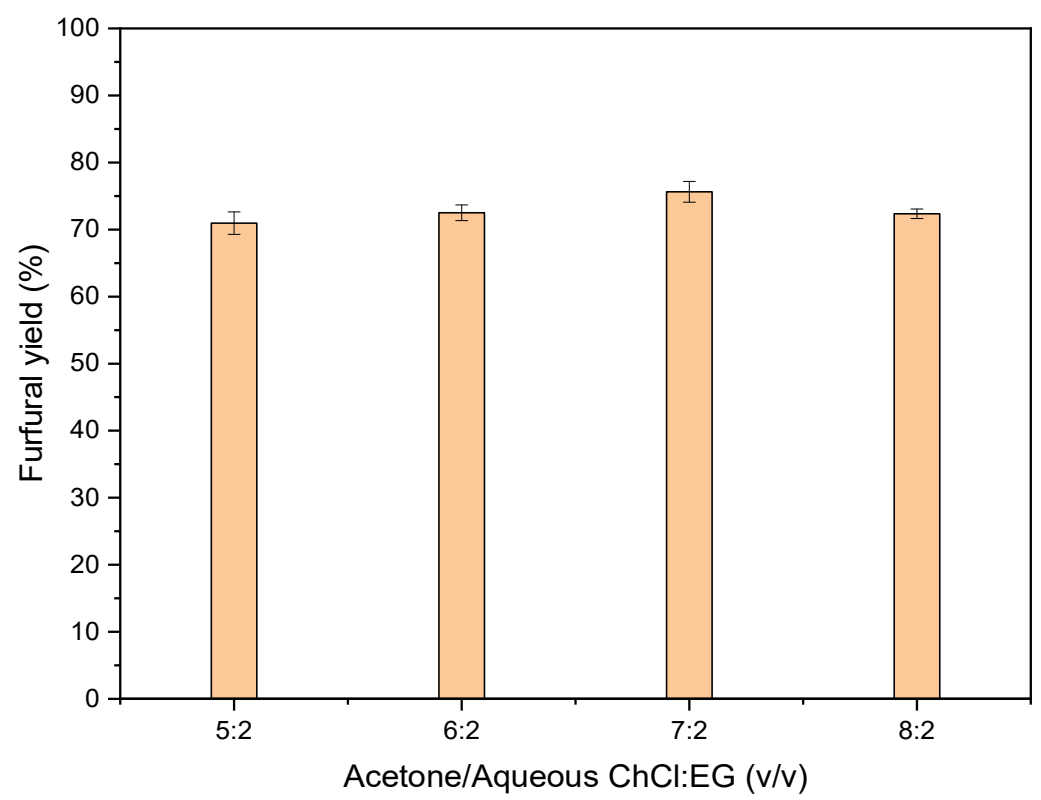

Figure 2.13 Effects of acetone to aqueous $\mathrm{ChCl}$ :EG ration on furfural production. 


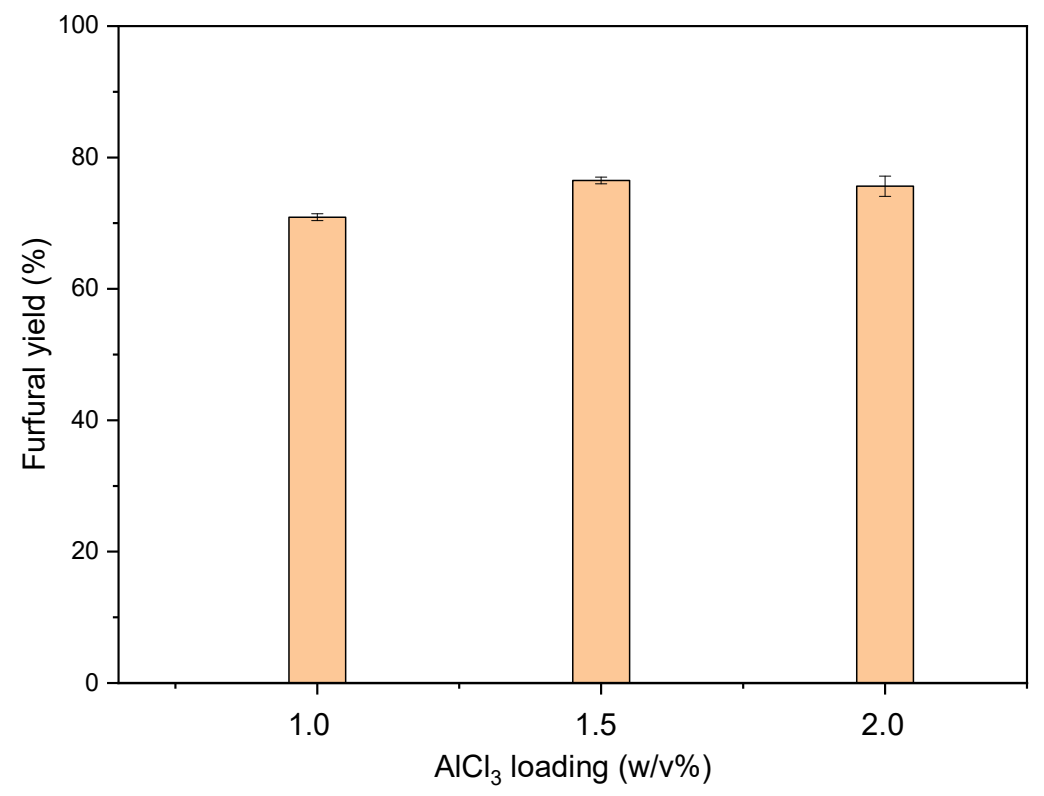

Figure 2.14 Effects of $\mathrm{AlCl}_{3}$ loading on furfural production.

Lastly, we tested the furfural production form the pretreatment liquor under the above conditions. As aforementioned, the pretreatment liquor contained aqueous $\mathrm{ChCl}$ :EG (1:2, 30\% water) and the xylose concentration in the liquor was $16.35 \mathrm{~g} / \mathrm{L}$. The tested biphasic reaction system was thus composed of the above model liquor and acetone with a volumetric ratio of $7: 2$. The furfural yield of $75 \%$ was obtained with more than $90 \%$ of the furfural partitioned into the acetone phase (Fig. 2.15). The use of acetone as an extraction solvent protects the furfural from degradation and enables facile recovery of furfural as acetone can be removed/recovered under reduced pressure even at ambient temperature. In addition to these advantages, the aldol condensations between furfural and acetone can lead to the formation of $\mathrm{C}_{13}-\mathrm{C}_{15}$ adducts, which can be upgraded into high quality fuels upon hydrodeoxygenation (Faba et al., 2012). This pathway has been deemed as a very promising route toward production of drop-in fuels (Faba et al., 2016). By using acetone as an extraction phase for furfural production from $\mathrm{C}_{5}$ sugars can possibly simplifies this pathway by 
eliminating the furfural recovery step. Overall, this novel biphasic system opens a new avenue for upgrading hemicellulose into platform chemicals and drop-in fuels.

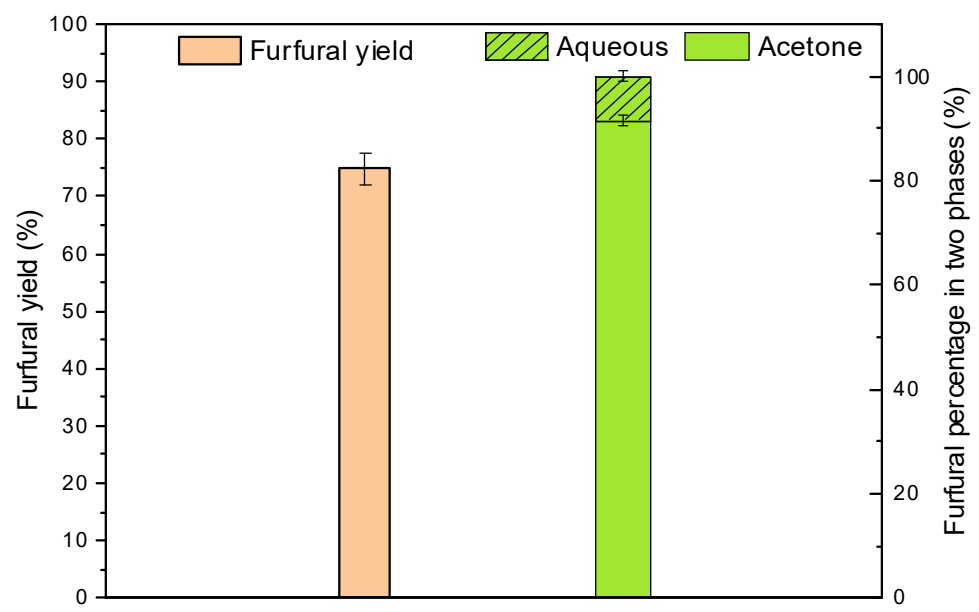

Figure 2.15 Furfural production in pretreatment liquor/acetone biphasic system.

\subsection{Conclusions}

This study demonstrated that acidified ChCl:EG was highly effective for lignin and hemicellulose dissolution while preserving most cellulose during switchgrass fractionation. Lignin properties are tunable by controlling the pretreatment conditions, water content and molar ratio of HBA to HBD. Tailoring the properties of lignin did not sacrifice the cellulose digestibility, and the highest sugar yield reached over $90 \%$ after enzymatic hydrolysis at $20 \%$ solid loading, corresponding to monomeric sugar concentration of $203 \mathrm{~g} / \mathrm{L}$. The DESs contained pretreatment liquor was rich in xylose and can form a biphasic system with acetone. This novel biphasic system enabled furfural production at a yield of $75 \%$, and was energetically advantageous to furfural recovery. In sum, this versatile DES system has great potential to sever as a platform solvent system for lignocellulosic biomass fractionation. 


\section{Disclosure}

The following chapter was based on the paper that has been published on the journal of ACS Sustainable Chemistry and Engineering. Reproduced with permission from Chen et al. (Chen et al., 2018a). Copyright (2018) American Chemical Society. Dr. Xianglan Bai and her graduate student Mr. Lusi A in Iowa State University are the coauthors, and contributed to the characterization of lignin using GPC, TGA, and pyrolysis GC-MS. 


\section{Chapter 3 High-solid Lignocellulose Processing Enabled by Natural Deep Eutectic Solvent for Lignin Extraction and Industrially Relevant Production of Renewable Chemicals}

\subsection{Introduction}

Biofuels and bio-based chemicals from lignocellulosic biomass can replace petroleum-based counterparts. For a biorefinery based on a biochemical conversion platform, a pretreatment step is often required to overcome the recalcitrance of lignocellulosic biomass and to facilitate the release of fermentable sugars. In the past two decades, great efforts have been devoted to developing a pretreatment that maximizes cellulose digestibility. However, the vast majority of lignin is treated as a waste and burned as low-grade solid fuel for biorefinery plants. To make biorefinery lignin a profitable product, one prerequisite is to extract valorizable lignin with high yield from lignocellulosic biomass. In addition to lignin, liquid waste should be minimized and post-treated/explored further for value-added conversion. Pretreatment solvent recycling would not only save costs for chemicals but also minimize waste stream generation. Another benefit with solvent recycling is enrichment of solubilized sugars in pretreatment liquor, which would make them concentrated enough for further upgrading, such as lipid and furfural production (Chen \& Wan, 2018). Therefore, a successful biorefinery should be enabled by economically viable and environmentally sustainable technologies to supply market-driven products (Sun et al., 2016b).

Using high solid loadings at $15 \%$ or above in the unit operations of lignocellulose conversion, such as pretreatment and enzymatic hydrolysis, could improve production efficiency, reduce energy consumption, ease downstream processing, reduce waste generation, and lower the overall processing cost 
(Modenbach \& Nokes, 2012). For ethanol production, an ethanol titer of $50 \mathrm{~g} / \mathrm{L}$ or above is desired for industrial scale, which requires at least $10 \%$ glucan loading for enzymatic hydrolysis (Chen et al., 2016; Nguyen et al., 2016). Similar scenario is applied to another versatile platform chemical - 2,3-butanediol (2,3-BD) which has a broad industrial application (Ji et al., 2011). Cellulosic 2,3-BD titers reported seldom exceeds $50 \mathrm{~g} / \mathrm{L}$ unless sugar hydrolysate is purposely concentrated prior to fermentation through costly membrane filtration or energy-intensive approach (Adlakha \& Yazdani, 2015; Ji et al., 2011; Um et al., 2017). With an increasing market "pull" for 2,3-BD, especially as a precursor for tire manufacturing, it is important to achieve more industrially relevant titer, yield, and productivity (TYP). However, high-solid loading enzymatic hydrolysis remains challenging due to substrate inhibition and mass transfer limitations (Ramachandriya et al., 2013). Likewise, limited mass transfer could arise from high solid loading pretreatment. Therefore, pursuing high solid loadings for both enzymatic hydrolysis and pretreatment would be more challenging but highly rewarding.

DESs are emerging designer solvents for lignocellulose pretreatment. Among the numerous DESs reported so far, natural DESs with biomass-derived polyols as hydrogen bond donors, especially choline chloride:ethylene glycol ( $\mathrm{ChCl}: \mathrm{EG})$, hold great promise for lignocellulosic biomass pretreatment due to its good solubility of lignin, low vapor pressure, and viscosity (Di Marino et al., 2016; Smith et al., 2014). In addition, $\mathrm{ChCl}$ :EG has been shown to stabilize enzymes and be compatible with microorganisms (Gunny et al., 2015; Hayyan et al., 2013; Kumar et al., 2016b), which provides a basis for designing one-pot biochemical reaction. Compared with other DESs (e.g., ChCl:Urea), ChCl:EG could also serve as an excellent medium for electrochemical depolymerization of lignin (Di Marino et al., 2016), suggesting the 
feasibility of adopting a simplified route for lignin valorization by integrating lignin extraction and upgrading in a single solvent. However, lignocellulose fractionation by ChCl:EG has been shown to be a slow process (Kumar et al., 2016b; Zhang et al., 2016a). Our previous study indicated that polyol-based DES (i.e., $\mathrm{ChCl}$ :Glycerol) became highly effective under an acidic environment which was much milder than conventional dilute acid pretreatment (Chen \& Wan, 2018). Acidic DESs composed of $\mathrm{ChCl}$ and carboxylic acids (e.g., formic acid, lactic acid) also showed impressive performance (Xu et al., 2016; Zhang et al., 2016a). Possible mechanism for enhanced delignification and hemicellulose removal by acidified/acidic DESs is the better availability and reactivity of acidic protons in a DES environment, which is critical for acid-catalyzed cleavage of numerous bonds (e.g., ether bonds, ester bonds, ligninpolysaccharide interlinkages) in lignocellulose complex. Another benefit of using polyol-based natural DES in acidic environment is avoiding possible side reactions, like esterification between carboxylic acid-based DESs and cellulose, which could limit cellulose digestibility to a great extent. Our studies on polyol-based natural DESs under acidic environment (also called acidified DESs) would broaden the spectrum of DESs for lignocellulose processing, identify high-performance DESbased pretreatment solvents, and provide insights into DES-enabled lignocellulose fractionation.

The objective of this study was to develop a high solid loading, $\mathrm{ChCl}$ :EG based pretreatment for viable lignocellulose processing. Using switchgrass as a model feedstock, the effects of acid, water, and solid loading on $\mathrm{ChCl}$ :EG pretreatment were studied. The solvent recyclability and reusability were also evaluated. Moreover, pretreated switchgrass was tested for concentrated sugar production and further conversion into 2,3-BD. We also investigated the enrichment of xylan-based sugars in 
pretreatment liquor upon solvent recycling and subsequent liquor upgrading into furfural.

\subsection{Experimental section}

\subsubsection{Materials.}

Switchgrass was collected from the South Farm at University of Missouri in Columbia, Missouri, USA. Raw switchgrass was air-dried, ground through a $2 \mathrm{~mm}-$ mesh screen, and stored in an airtight container. It contained $34.5 \%$ glucan, $23.3 \%$ xylan, and 20.4\% lignin. Hydrolytic enzymes (Cellic ${ }^{\circledR}$ CTec2 and HTec2) were kindly provided by Novozyme (Franklinton, NC, USA). All the chemicals were purchased from Fisher Scientific (Hampton, NH, USA).

\subsubsection{DES Synthesis}

$\mathrm{ChCl}$ was dried at $80^{\circ} \mathrm{C}$ for $6 \mathrm{~h}$ and cooled to room temperature in a desiccator prior to use. Neat $\mathrm{ChCl}$ :EG was synthesized by mixing $\mathrm{ChCl}$ and $\mathrm{EG}$ at a molar ratio of $1: 2$ and heating the mixture at $60^{\circ} \mathrm{C}$ with continuous stirring (200 rpm) until a transparent solvent was formed. Aqueous $\mathrm{ChCl}$ :EG containing $10 \mathrm{wt} \%$ and 20 $\mathrm{wt} \%$ water was prepared by adding deionized (DI) water to neat $\mathrm{ChCl}: \mathrm{EG}$. Acidified neat or aqueous $\mathrm{ChCl}$ :EG contained $1.0 \mathrm{wt} \% \mathrm{H}_{2} \mathrm{SO}_{4}$.

\subsubsection{DES pretreatment and lignin recovery}

For DES pretreatment, $10 \mathrm{~g}$ of dry switchgrass was mixed with acidified neat or aqueous $\mathrm{ChCl}: \mathrm{EG}$ in a $250 \mathrm{~mL}$ glass bottle. The bottle was then placed in a preheated oil bath with manual mixing every 5 min using a PTFE rod. The pretreatment with $10 \mathrm{wt} \%$ solid loading was conducted at $130{ }^{\circ} \mathrm{C}$ for $30 \mathrm{~min}$. For the pretreatment at high solid, two conditions were tested: (a) $130{ }^{\circ} \mathrm{C}, 30 \mathrm{~min}, 20 \mathrm{wt} \%$ solid loading (P20); and (b) $130^{\circ} \mathrm{C}, 45 \mathrm{~min}, 27 \mathrm{wt} \%$ solid loading (P27). At the end of pretreatment, the bottle was taken out of the oil bath immediately, and cooled to room 
temperature. Then, $50 \mathrm{~mL}$ of acetone:water $(1: 1, \mathrm{v} / \mathrm{v})$ was added to the bottle followed by vacuum filtration. The solid residue was washed with $25 \mathrm{~mL}$ of acetone:water (1:1, $\mathrm{v} / \mathrm{v})$ four times and then stored at $-20^{\circ} \mathrm{C}$ for further use. Acetone:water $(1: 1, \mathrm{v} / \mathrm{v})$ was chosen as a washing solvent as this mixture is miscible with $\mathrm{ChCl}: \mathrm{EG}$, and acetone can be easily removed via vacuum evaporation or recovered via distillation due to its low boiling point.

The filtrate collected from the slurry separation and the washing of the pretreated solids were combined and evaporated to remove acetone via vacuum evaporation. Lignin was precipitated after acetone removal, collected via centrifugation at $10,000 \times g$ for $5 \mathrm{~min}$, and further washed with $15 \mathrm{~mL}$ of ethanol:water $(1: 9, \mathrm{v} / \mathrm{v})$ four times. Lastly, lignin was dried at $45^{\circ} \mathrm{C}$ in a convection oven for $12 \mathrm{~h}$ and stored in an airtight glass container at room temperature. To recycle CC-EG, the above liquor was evaporated at $70^{\circ} \mathrm{C}$ in a convection oven to remove excess water. The recycled CC-EG was supplemented with acid to make its $\mathrm{pH}$ equal to that of the freshly prepared acidified $\mathrm{ChCl}: \mathrm{EG}$, and then used for a new pretreatment cycle. Lignin yield was defined as lignin recovered from a single pretreatment cycle with respect to lignin solubilized from the same cycle. Cumulative lignin yield was calculated based on cumulative pretreatment cycles.

\subsubsection{Enzymatic hydrolysis}

Enzymatic hydrolysis was conducted at $50{ }^{\circ} \mathrm{C}$ and $150 \mathrm{rpm}$ for $24 \mathrm{~h}$, unless otherwise stated. CTec2 and HTec2 were loaded at 20 and $2 \mathrm{mg}$ protein/g solid, respectively, to citrate buffer (final concentration of $50 \mathrm{mM}, \mathrm{pH} 5.5$ ). The $\mathrm{pH}$ value of 5.5 was selected for enzymatic hydrolysis as it is within the optimum range (pH 5.05.5) for $\mathrm{CTec} 2$ and would minimize the alkaline consumption for bringing $\mathrm{pH}$ to a higher level ( $\mathrm{pH} 7$ ) for 2,3-BD fermentation. Low solid loading was $2 \mathrm{wt} \%$ and high 
solid loadings were $20 \mathrm{wt} \%$ and $25 \mathrm{wt} \%$. The hydrolysate was boiled for $5 \mathrm{~min}$ to deactivate the enzymes and then centrifuged at $17,000 \times g$ for $2 \mathrm{~min}$. The supernatant was collected and stored at $-20^{\circ} \mathrm{C}$ prior to analysis. Sugar yield was defined as a percentage of theoretical sugar yield of untreated/pretreated biomass. All tests were run at least in duplicate. The data were reported as mean values with standard deviation.

\subsubsection{2,3-BD fermentation}

Bacillus vallismortis (NRRL B-14891) used for 2,3-BD fermentation was kindly provided by the United States Department of Agriculture (USDA) Agriculture Research Service (ARS) Culture Collection (Peoria, IL). The strain was routinely maintained on Luria-Bertani (LB) agar plate containing (g/L): tryptone 10 , yeast extract $5, \mathrm{NaCl} 10$, and agar 15 . The seed culture was prepared by growing the strain in $50 \mathrm{~mL}$ of $\mathrm{LB}$ medium for $12 \mathrm{~h}$ at $30{ }^{\circ} \mathrm{C}$ and $200 \mathrm{rpm}$. The cellular biomass was collected via centrifugation at 5,000 $\times g$ for $5 \mathrm{~min}$, washed twice with $0.9 \%$ saline solution, and then re-suspended in the saline solution. The re-suspended cells were then inoculated into $20 \mathrm{~mL}$ of fermentation medium to obtain an initial optical density (OD) at $600 \mathrm{~nm}$ of 0.2 . The fermentation medium contained (g/L): $\mathrm{KH}_{2} \mathrm{PO}_{4} 4$, tryptone 5, yeast extract $10,\left(\mathrm{NH}_{4}\right)_{2} \mathrm{SO}_{4} 1, \mathrm{MgSO}_{4} \cdot 7 \mathrm{H}_{2} \mathrm{O} 0.75$, and $\mathrm{CaCl}_{2} \cdot 2 \mathrm{H}_{2} \mathrm{O} 0.1$. The hydrolysate resulting from $20 \%$ and $25 \%$ solid loading enzymatic hydrolysis of switchgrass pretreated at 20\% solid loading, as described in Section 3.2.4, was used as the substrate. The $\mathrm{pH}$ of fermentation media was adjusted to 7 every $12 \mathrm{~h}$ using $5 \mathrm{M}$ $\mathrm{NaOH}$. The $\mathrm{pH}$ probe was sterilized with $70 \%$ ethanol, and dried under an aseptic condition in a biological safety chamber before use. The fermentation was conducted in $125 \mathrm{~mL}$ Erlenmeyer flask plugged with foam stopper in an incubator shaker at $30{ }^{\circ} \mathrm{C}$ and $200 \mathrm{rpm}$ for $72 \mathrm{~h}$. The samples were taken every $12 \mathrm{~h}$ and analyzed for the 
titers of sugar and 2,3-BD using HPLC. To compensate water loss due to evaporation, sterile DI water was added every $12 \mathrm{~h}$ before sampling and $\mathrm{pH}$ adjustment. The fermentation tests were run at least in duplicate. The data were reported as mean values with standard deviation.

\subsubsection{Furfural production}

Furfural production was conducted in a pressure tube using a biphasic reaction system comprising $1 \mathrm{~mL}$ of aqueous sample and $4 \mathrm{~mL}$ methyl isobutyl ketone (MIBK). The pressure tube was placed in a preheated oil bath at $160{ }^{\circ} \mathrm{C}$ for $40 \mathrm{~min}$. Aqueous CC-EG (50 wt\% water) containing $100 \mathrm{~g} / \mathrm{L}$ xylose was first used for the determination of optimal $\mathrm{AlCl}_{3}$ loading. A varying level of $\mathrm{AlCl}_{3}$ ranging from 0.38 to $3.00 \% \mathrm{w} / \mathrm{v}$ loading was tested. The pretreatment liquor was tested as both a reaction medium and substrate for furfural production under the same reaction condition with the optimized $\mathrm{AlCl}_{3}$ loading.

\subsubsection{Analytical methods}

The compositional analysis, lignin purity analysis, and glucose/xylose oligomers determination in pretreatment liquor were conducted following NREL laboratory protocols (Sluiter et al., 2006; Sluiter et al., 2008). Lignin purity was calculated as the total amount of acid soluble lignin (ASL) and acid insoluble lignin (AIL) relative to the dry mass of recovered lignin. Sugar concentration was determined using a high performance liquid chromatography (HPLC) Agilent 1200 series (Agilent Technologies, Palo Alto, USA) equipped with a refractive index detector (RID) and a HPX-87P column $(300 \times 7.8 \mathrm{~mm})($ Bio-Rad, Hercules, USA) as described in our previous study (Chen \& Wan, 2018).

Furfural titers in both aqueous phase and MIBK phase were determined using the above HPLC system but equipped with a ZORBAX Eclipse XDB-C18 column 
$(150 \times 4.6 \mathrm{~mm})($ Agilent Technologies, Palo Alto, USA). The mobile phase was 20 vol\% methanol, eluting at a flow rate of $1 \mathrm{~mL} / \mathrm{min}$. The temperatures of the RID and column were maintained at 30 and $35^{\circ} \mathrm{C}$ respectively. For the determination of 2,3BD and EG, HPX-87H column $(300 \times 7.8 \mathrm{~mm})($ Bio-Rad, Hercules, USA) was used. The temperatures of the RID and column were kept at 40 and $65^{\circ} \mathrm{C}$, respectively. $\mathrm{H}_{2} \mathrm{SO}_{4}(5 \mathrm{mM})$ was used as the mobile phase with a flow rate of $0.6 \mathrm{~mL} / \mathrm{min}$.

Powder X-ray diffraction (XRD) data were collected on a Scintag X2 diffractometer (Scintag, Inc., Cupertino, $\mathrm{CA}$ ) equipped with a $\mathrm{Cu} / \mathrm{K} \alpha$ sealed tube $\mathrm{X}$ ray generator (operated at $45 \mathrm{kV}, 40 \mathrm{~mA}$ ). Scan was collected from $2 \theta$ of 5 to $50^{\circ}$ at a scanning rate of $2^{\circ} / \mathrm{min}$ and a resolution of $0.02^{\circ}$ per data point. Crystallinity index (CrI) was calculated based on the following equation:(Segal et al., 1959) $\mathrm{CrI}(\%)=$ $\left[\left(\mathrm{I}_{002}-\mathrm{I}_{\mathrm{am}}\right) / \mathrm{I}_{002}\right] \times 100$, where $\mathrm{I}_{002}$ is the maximum intensity of diffraction peak at $2 \theta$ of $22^{\circ} ; \mathrm{I}_{\mathrm{am}}$ is the minimum intensity of the amorphous portion at $2 \theta$ of $18.0-19.0^{\circ}$.

Functional groups of the biomass samples were analyzed using attenuated total reflection Fourier transform infrared spectroscopy (ATR-FTIR) (Nicolet 4700, Thermo Electron, USA). Sample spectra were obtained via 64 scans over the range between 400 and $4000 \mathrm{~cm}^{-1}$.

Extractive-free and ball-milled switchgrass was enzymatically hydrolyzed for 48 $\mathrm{h}$, and then subjected to a mild acidolysis using dioxane:water $(96: 4, \mathrm{v} / \mathrm{v})$ containing $0.04 \mathrm{~N} \mathrm{HCl}$ for $24 \mathrm{~h}$. The mixture was centrifuged, and the supernatant was collected and neutralized with sodium bicarbonate. The neutralized supernatant was drop-wise added to cold DI water, and the precipitation was recovered as crude lignin. The crude lignin was further purified by dissolving in acetic acid:water $(9: 1, \mathrm{v} / \mathrm{v})$, followed by precipitation in cold DI water. The precipitated lignin was freeze-dried, and dissolved in 1,2-dichloroethane:ethanol mixture $(2: 1, \mathrm{v} / \mathrm{v})$ and precipitated in diethyl ether. The 
lignin was dried overnight in a vacuum oven overnight to obtain the native lignin. 2D HSQC NMR was conducted on a Bruker AVIII $800 \mathrm{MHz}$ spectrometer using the Bruker-supplied pulse sequence named hsqcetgp (Bruker, Billerica, MA). The spectral width is $15.4 \mathrm{ppm}$ for ${ }^{1} \mathrm{H}$ and $170.0 \mathrm{ppm}$ for ${ }^{13} \mathrm{C}$. A total of $2048\left({ }^{1} \mathrm{H}\right)$ complex points were acquired with $256\left({ }^{13} \mathrm{C}\right)$ time increments. The ${ }^{1} \mathrm{~J}_{\mathrm{CH}}$ was set to be $145 \mathrm{~Hz}$ which is the average one-bond $\mathrm{C}-\mathrm{H}$ coupling constant. The number of scan was 48 , and the repetition delay was $1.5 \mathrm{~s}$. The ${ }^{13} \mathrm{C}$ dimension was zero-filled to 2048 points before the data were subjected to a sine-squared window function (shifted $90^{\circ}$ ) and Fourier transformation. The chemical shift axes were calibrated with respect to the solvent signals (2.49 ppm for residual proton and $39.5 \mathrm{ppm}$ for $\left.{ }^{13} \mathrm{C}\right)$.

2D HSQC NMR analysis of native lignin and lignin resulting from DES pretreatment was conducted as described in our previous study (Chen et al., 2018b). Native lignin was prepared and purified as described previously (Zhou et al., 2016b). The detailed procedures were provided in the Supporting Information. $P$-coumarate $(p \mathrm{CA})$, ferulate (FA), and $\beta-\mathrm{O}-4$ abundance were estimated semi-quantitatively using the volume integration of contours in HSQC spectra (Ragauskas et al., 2014a).

Gel permeation chromatography (GPC) was performed to determine the molecular weight distribution of lignin. Prior to GPC analysis, the lignin samples were acetylated to increase their solubility in tetrahydrofuran (THF). For acetylation, $100 \mathrm{mg}$ of lignin along with $3 \mathrm{~mL}$ pyridine and $3 \mathrm{~mL}$ acetic anhydride were added to a glass vial. The vial was then heated in oil bath at $80{ }^{\circ} \mathrm{C}$ for $3 \mathrm{~h}$ with continuous stirring. After acetylation, $500 \mathrm{~mL}$ of DI water was added to the solution to precipitate acetylated lignin. The precipitated lignin was washed with DI water for three times to remove residual chemicals. To prepare GPC sample, $20 \mathrm{mg}$ of acetylated lignin was dissolved in $10 \mathrm{~mL}$ THF and then purified using a $0.45 \mu \mathrm{m}$ filter to remove any solid 
particles. The GPC analysis was performed in a Dionex Ultimate 3000 series high performance liquid chromatography (HPLC) equipped with a Shodex Refrative Index (RI) detector and Diode Array Detector (DAD). Two Agilent PLgel $3 \mu \mathrm{m}$ 100A0 300 $\times 7.5 \mathrm{~mm}(\mathrm{p} / \mathrm{n}$ PL1110-6320) were connected in series in the HPLC and the flow rate of THF was $1 \mathrm{~mL} / \mathrm{min}$. The GPC column was pre-calibrated with polystyrene standards with molecular weights from 162 to $45120 \mathrm{~g} / \mathrm{mol}$, and ultraviolet wave length of $254 \mathrm{~nm}$ was used to analyze the molecular weight distribution. The GPC analysis was duplicated for each lignin sample.

Thermal stability of lignin was determined using a Mettler Toledo thermogravimetric analysis system (TGA/DSC 1 STAR system). Approximately 20 mg of lignin sample was placed in a crucible and heated under a nitrogen environment from room temperature to $105^{\circ} \mathrm{C}$ at $10{ }^{\circ} \mathrm{C} / \mathrm{min}$ and then hold for $40 \mathrm{~min}$ to remove the moisture. The sample was continuously heated to $900{ }^{\circ} \mathrm{C}$. The analysis was duplicated for each lignin sample.

Fast pyrolysis of lignin was conducted using a Frontier Lab Tandem micropyrolyzer (Tx-3050 TR) with an auto sampler. The configuration of the reactor is described in previous literature.(Zhou et al., 2016b) Briefly, the micro-pyrolyzer consists of two furnaces connected in series. During the tests, $500 \mu \mathrm{g}$ of lignin was placed in a deactivated stainless-steel cup and pyrolyzed in the top furnace at $500{ }^{\circ} \mathrm{C}$ using helium as a carrier gas. The temperature of the lower furnace was maintained at $350{ }^{\circ} \mathrm{C}$ to prevent the condensation of the pyrolysis vapor. The pyrolysis vapors leaving the micro-pyrolyzer were directly swept into Agilent 7890B GC/MS-FIDTCD for instantaneous product analysis. Helium was also used as the purge gas in the GC. The flow rate of helium was $156 \mathrm{~mL} / \mathrm{min}$ and the split ratio at the front-inlet was 50:1. The temperature of the GC oven was kept at $40{ }^{\circ} \mathrm{C}$ for $3 \mathrm{~min}$, and then increased 
to $280{ }^{\circ} \mathrm{C}$ at a heating rate of $6{ }^{\circ} \mathrm{C} / \mathrm{min}$. Finally, the $\mathrm{GC}$ was held at $280{ }^{\circ} \mathrm{C}$ for $3 \mathrm{~min}$. Two Phenomenex ZB $1701(60 \mathrm{~m} \times 0.250 \mathrm{~mm} \times 0.250 \mu \mathrm{m})$ columns were used for the MS and FID, and a Porous Layer Open Tubular (PLOT) $(60 \mathrm{~m} \times 0.320 \mathrm{~mm})$ was used for the TCD. The monomers identified by the MS were quantified by FID. The FID calibration curves were created by injecting five different concentrations of each compound into the GC. The pyrolysis test was triplicated for each lignin sample and the averaged results were reported.

\subsection{Results and discussion}

\subsubsection{Effects of acidification on the pretreatment performance of $\mathrm{ChCl}: \mathrm{EG}$}

The pretreatment effectiveness of acidified $\mathrm{ChCl}: \mathrm{EG}$ was compared with neat ChCl:EG, acidified EG, and dilute acid solution. As shown in Figure 3.1 and 3.2, neat CC-EG removed $24 \%$ of lignin but had minor effects on glucan and xylan, indicating the high selectivity of $\mathrm{ChCl}: \mathrm{EG}$ on lignocellulose fractionation. Similar results were also reported by a prior study using $\mathrm{ChCl}$ :EG to pretreat corncob (Zhang et al., 2016a). Once $\mathrm{ChCl}: \mathrm{EG}$ was acidified, lignin and xylan removal increased to about $87 \%$ and $82 \%$ (Figure 3.1 ), respectively. Although acid solution only $\left(1.0 \% \mathrm{H}_{2} \mathrm{SO}_{4}\right)$ led to more xylan and lignin removal than neat $\mathrm{ChCl:EG}$, its performance was not comparable with acidified $\mathrm{ChCl}$ :EG. In consistence with prior study (Zhang \& Yu, 2013), acidified EG was effective for lignin and xylan removal, demonstrating a similar performance to acidified $\mathrm{ChCl}$ :EG with regard to lignin and xylan removal. These results suggested a strong synergism between acid and $\mathrm{ChCl}$ :EG in the pretreatment of switchgrass. Similar synergism has also been demonstrated by several organic solvent systems, such as $\gamma$-valerolactone, dioxane, and tetrahydrofuran (Mellmer et al., 2014; Nguyen et al., 2016; Shuai et al., 2016b). Comparing different solvent systems especially under a mild reaction condition, $\mathrm{H}_{2} \mathrm{SO}_{4}$ may be less active 
in water to donate proton for bond cleavage due to the solvation of acidic proton by water molecule (Mellmer et al., 2014). Similar to the aforementioned organic solvent systems, $\mathrm{ChCl}$ :EG should provide a favorable environment to make acidic proton more available and reactive for lignocellulose fractionation.

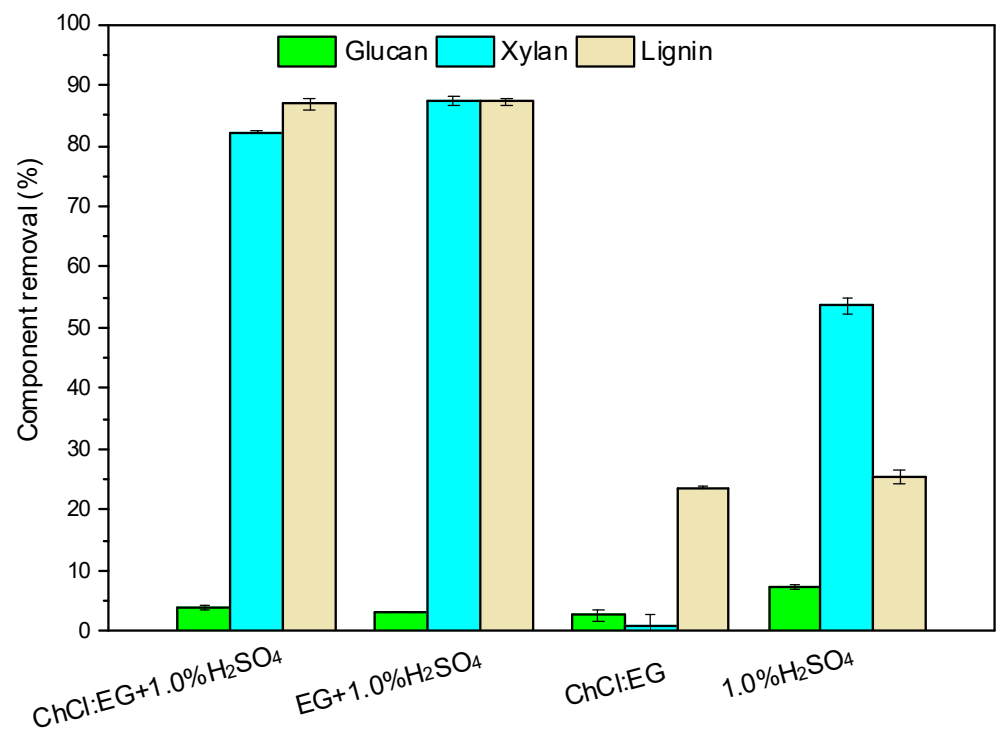

Figure 3.1 Switchgrass component removal in response to the pretreatment using different solvents.

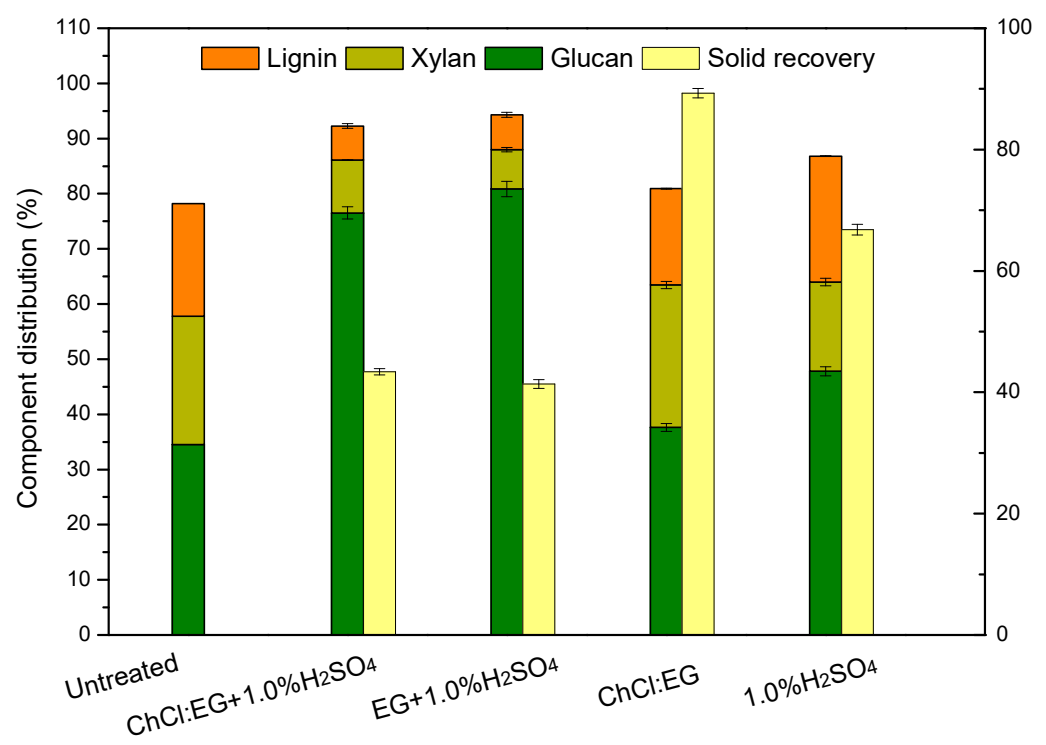

Figure 3.2 Switchgrass major component distribution and solid recovery in response to the pretreatment using different solvents. 
$\mathrm{XRD}$ analysis was done to understand the structural change of switchgrass in response to different pretreatments. As shown in Figure 3.3, switchgrass pretreated with neat $\mathrm{ChCl}$ :EG had a $\mathrm{CrI}$ of 54, which was same as that of the untreated one. For $\mathrm{H}_{2} \mathrm{SO}_{4}$ only-pretreated, acidified EG and acidified $\mathrm{ChCl}$ :EG-pretreated switchgrass, the $\mathrm{CrI}$ increased to 62,71 , and 66 , respectively, indicating increased cellulose contents after pretreatment. The changes in surface functional groups of switchgrass in response of pretreatment was revealed by FTIR (Figure 3.4). Typical peaks of cellulose were found at $900 \mathrm{~cm}^{-1}$ (C-H deformation in cellulose), $1100 \mathrm{~cm}^{-1}$ (C-O vibration in crystalline cellulose), 1035 and $1056 \mathrm{~cm}^{-1}$ (C-O stretching of cellulose), and $1160 \mathrm{~cm}^{-1}$ (C-O-C asymmetric stretching in cellulose) (Bekiaris et al., 2015). These cellulose peaks showed a higher intensity in acidified EG and $\mathrm{ChCl}: \mathrm{EG}$ pretreated switchgrass, suggesting a enrichment of cellulose in the sample. Characteristic peaks related to lignin, such as $1510 \mathrm{~cm}^{-1}$ (aromatic skeletal vibrations) and $1240 \mathrm{~cm}^{-1}$ (C-O vibration in the syringyl ring), was evident in the untreated, $\mathrm{ChCl}$ :EG pretreated, and acid only pretreated switchgrass, but became much weaker in the acidified EG and $\mathrm{ChCl}$ :EG pretreated ones, suggesting a lower lignin content in these samples (Ninomiya et al., 2012). 


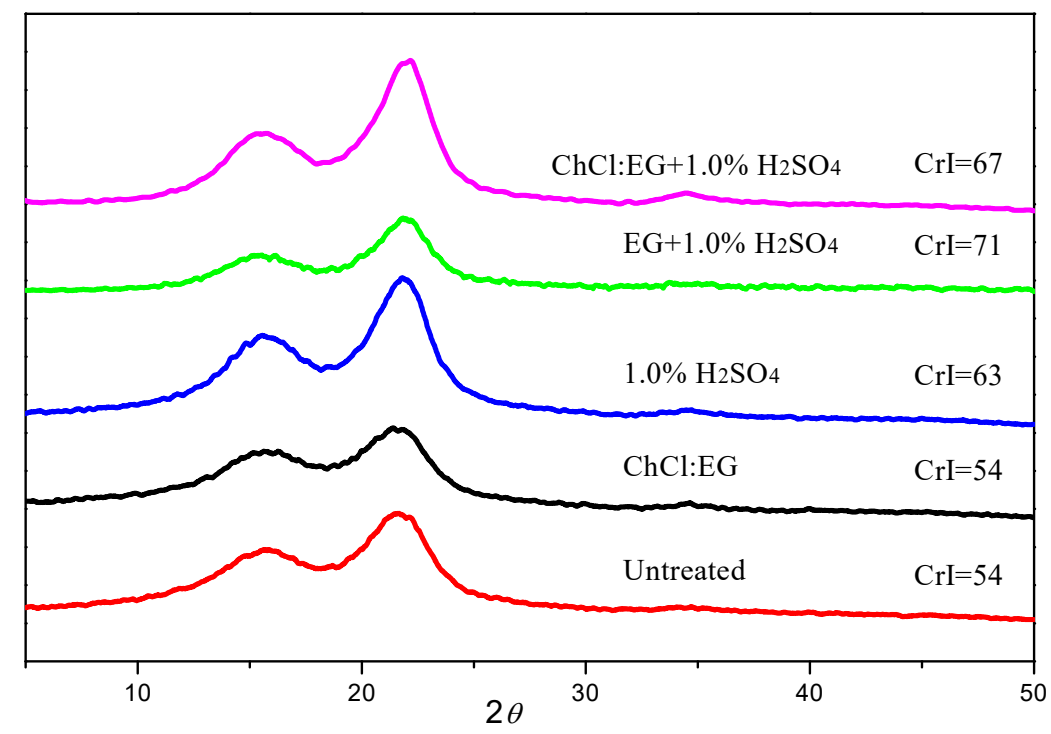

Figure 3.3 XRD pattern of pretreated switchgrass.

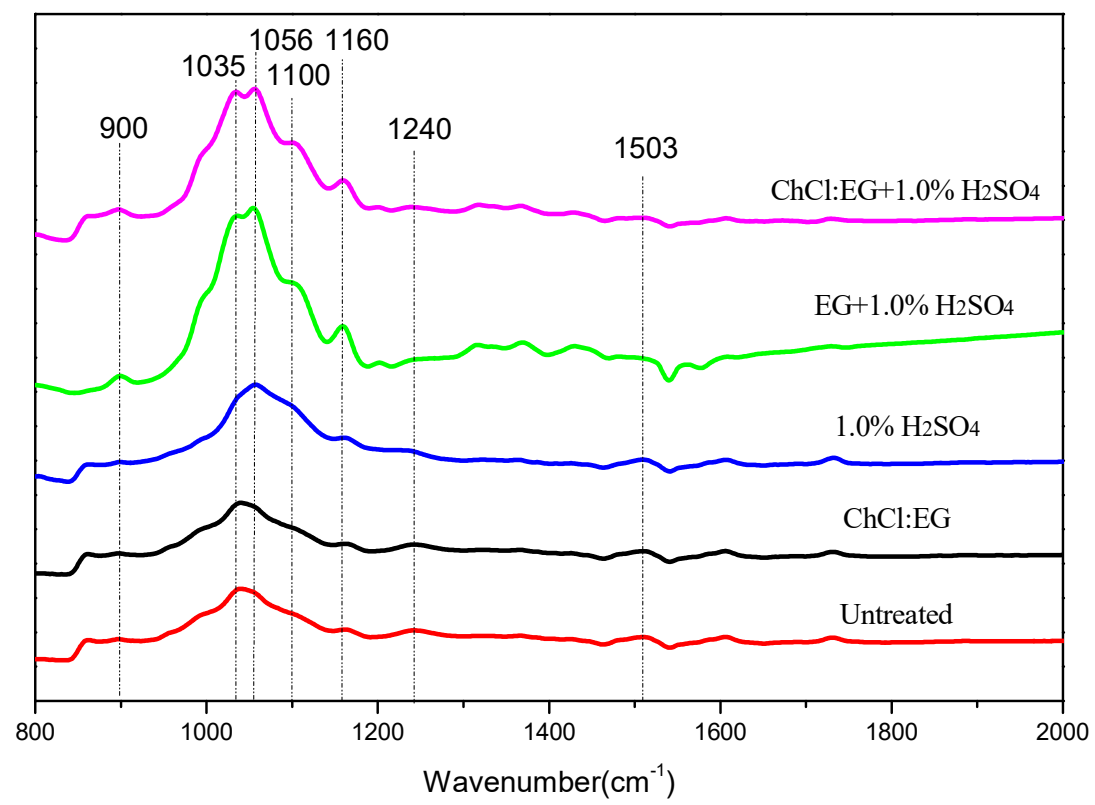

Figure 3.4 FTIR spectra of pretreated switchgrass.

The digestibility of pretreated switchgrass was evaluated by conducting enzymatic hydrolysis with $2 \mathrm{wt} \%$ solid loading for $24 \mathrm{~h}$. Switchgrass pretreated with neat $\mathrm{ChCl}$ :EG had glucose and xylose yields of $11 \%$ and $2 \%$, respectively, which 
were similar to those of the untreated switchgrass (Figure 3.5.). Acid only pretreatment $\left(1 \% \mathrm{H}_{2} \mathrm{SO}_{4}\right)$ improved the glucose yield from $11 \%$ to $37 \%$ and the xylose yield from $2 \%$ to $25 \%$. In contrast, both acidified $\mathrm{EG}$ and $\mathrm{ChCl}$ :EG resulted in a near theoretical sugar yield (Figure 3.5). Such high sugar yields could be attributed to the significant removal of xylan and lignin, which enhanced the accessibility of cellulose to hydrolytic enzymes. The highest glucose yield reported so far via DESs pretreatment was $99 \%$ for corn stover pretreated by $\mathrm{ChCl}$ :formic acid for $3 \mathrm{~h}$ at $130{ }^{\circ} \mathrm{C}$ using $5 \%$ solid loading (Xu et al., 2016). Other DESs, including ChCl:urea, $\mathrm{ChCl}$ :glycerol, $\mathrm{ChCl}$ :acetic acid, $\mathrm{ChCl}$ :oxalic acid, and $\mathrm{ChCl}$ :malonic, gave glucose yields much lower than $90 \%$ even under similar pretreatment conditions (Xu et al., 2016). Over $90 \%$ glucose yield of $\mathrm{ChCl}$ :Glycerol-pretreated corn cob was reported by Procentese et al. (Procentese et al., 2015), but the pretreatment required a quite severe condition $\left(150{ }^{\circ} \mathrm{C}\right.$ for $\left.15 \mathrm{~h}\right)$ even at $6 \%$ solid loading. Acidified $\mathrm{ChCl}: \mathrm{EG}$ outperformed most DES pretreatments in terms of pretreatment efficiency and effectiveness as well as biomass digestibility. In addition, the pretreatment based on acidified $\mathrm{ChCl}$ :EG required less severe conditions (shorter time and/or lower temperature), while the solid loading was comparable with or even higher than most reported DES pretreatments (Procentese et al., 2015; Xu et al., 2016). Overall, acidified $\mathrm{ChCl}$ :EG was highly efficient for xylan and lignin removal, and the resultant cellulose-enriched residue/pulp was highly digestible as discussed in the latter section. 


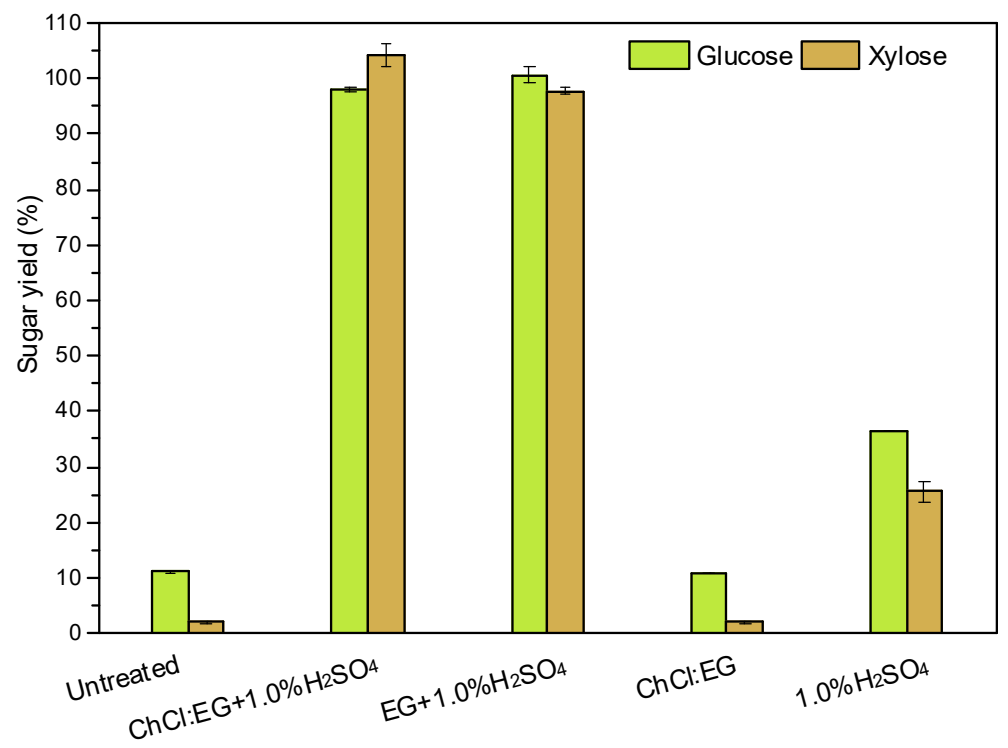

Figure 3.5 Switchgrass digestibility in response to the pretreatment using different solvents.

\subsubsection{High-solid loading pretreatment}

Since acidified $\mathrm{ChCl}$ :EG was able to substantially remove lignin and subsequently increase the digestibility of switchgrass, its performance on the pretreatments with a much higher solid loading (i.e., 20\%, 27\%) was explored. Acidified ChCl:EG still showed good performance at 20\% and 27\% loading, although lignin and xylan removal decreased with an increase in solid loading for pretreatment (Figure 3.6\&3.7). Some prior studies have demonstrated that water addition could lower the viscosity of DESs, enhance lignin extraction, and reduce cellulose loss (Hou et al., 2017; Kumar et al., 2016a). Consequently, the effects of water addition on the pretreatment performance of acidified $\mathrm{ChCl}$ :EG were also investigated. For $20 \%$ solid loading pretreatment, water addition at $10 \%$ and $20 \%$ appeared to not impact the fractionation performance of acidified $\mathrm{ChCl}: \mathrm{EG}$, since similar lignin and xylan removal, about $80 \%$ and $75 \%$, respectively, were achieved. In contrast, at a higher solid loading (27\%), acidified, aqueous $\mathrm{ChCl}$ :EG containing $10 \%$ water removed smaller amounts of lignin and xylan, but with similar degrees to acidified, neat 
ChCl:EG (Figure 3.6). At the same solid loading, acidified, aqueous $\mathrm{ChCl}$ :EG containing more water (20\%) solubilized less lignin and xylan from switchgrass.

Under all the circumstances, more than $95 \%$ of glucan was preserved. In consistence with the results for 10\% solid loading pretreatment as discussed in Section 3.3.1, acidified $\mathrm{ChCl}: \mathrm{EG}$ selectively removed lignin and xylan while preserving most cellulose.

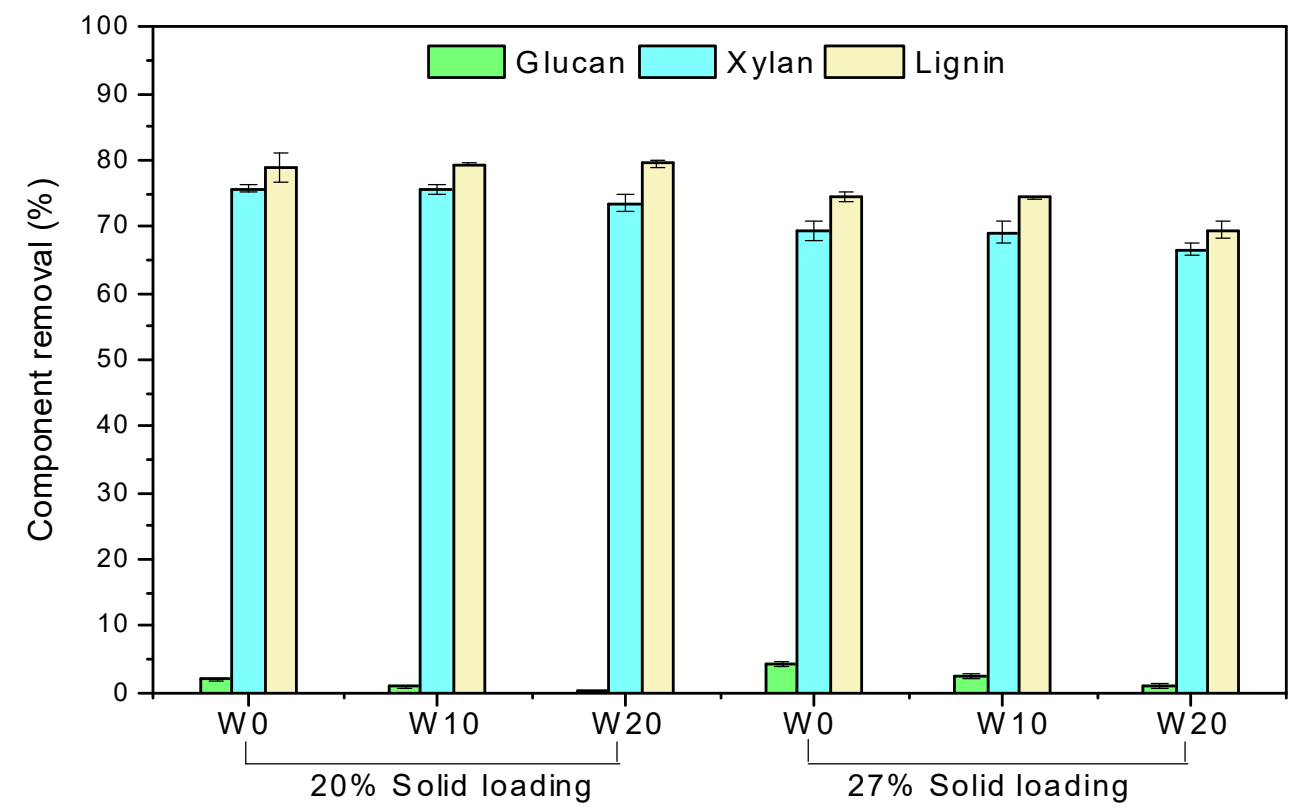

Figure 3.6 Switchgrass component removal and digestibility in response to $\mathrm{ChCl}$ :EG pretreatment under acidic condition with varying water addition and solid loadings. 


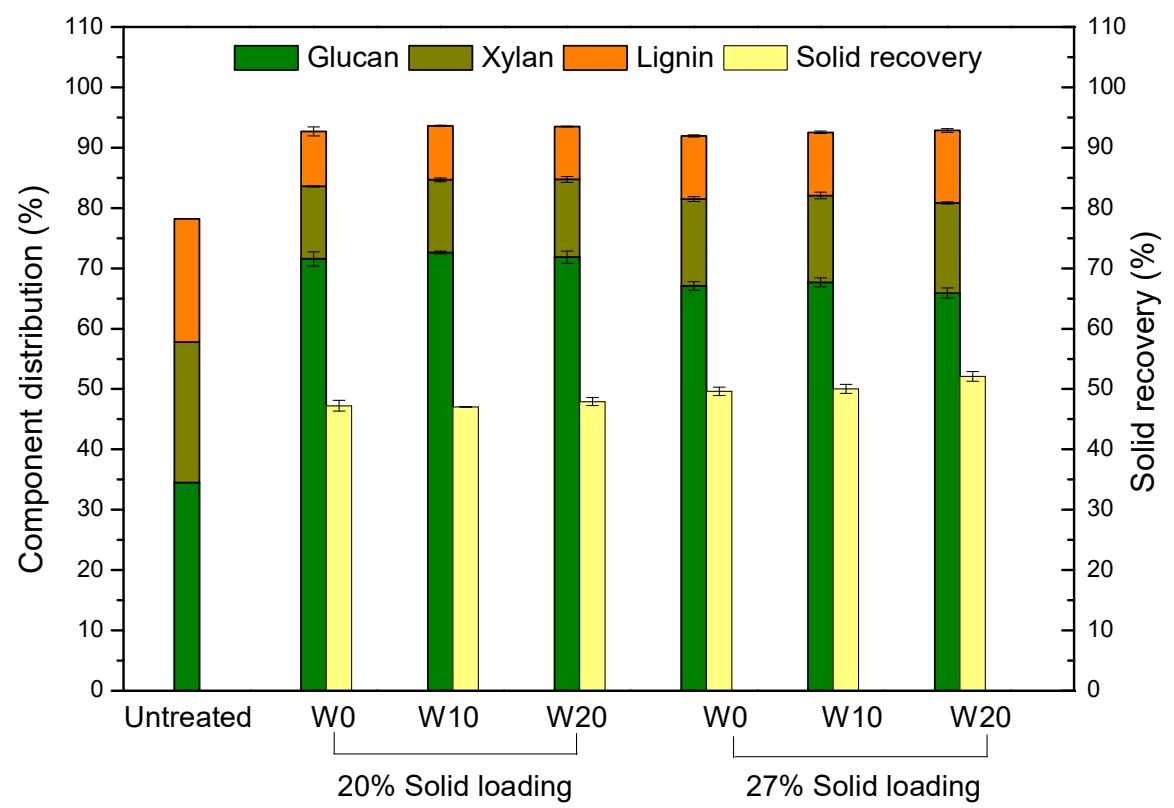

Figure 3.7 Switchgrass major component distribution and solid recovery in response to the pretreatment using acidified $\mathrm{ChCl}$ :EG with a varying water addition and solid loading.

Given significant xylan and lignin removal at high solid loading, it was expected that the sugar yield could be greatly enhanced. As shown in Figure 3.8, the glucose yields showed little variation in response to water addition during the pretreatment at $20 \%$ solid loading, reaching about $95 \%$. It is suggested that the digestibility of pretreated biomass should be largely dependent on the degree of delignification and/or the properties of lignin remaining in pretreated biomass ( $\mathrm{Li}$ et al., 2010). For example, switchgrass with a delignification over $60 \%$ via either ionic liquid pretreatment or alkaline pretreatment gave a glucose yield above 95\% after 24 h enzymatic hydrolysis (Karp et al., 2015; Li et al., 2010). Given that more than 70\% lignin was removed from switchgrass during all the pretreatment tested at $20 \%$ solid, it was not surprising to find that all the subsequently glucose yields reached near $95 \%$ with little variation. When the solid loading for pretreatment increased to $27 \%$, the glucose yield remained above $90 \%$ but was $2-5 \%$ lower than those from $20 \%$ solid loading pretreatment (Figure 3.8). Compared with glucose yields, xylose yields were 
near $10 \%$ lower, reaching approximately $85 \%$ and $80 \%$ for $20 \%$ and $27 \%$ solid loading pretreatments, respectively (Figure 3.8).

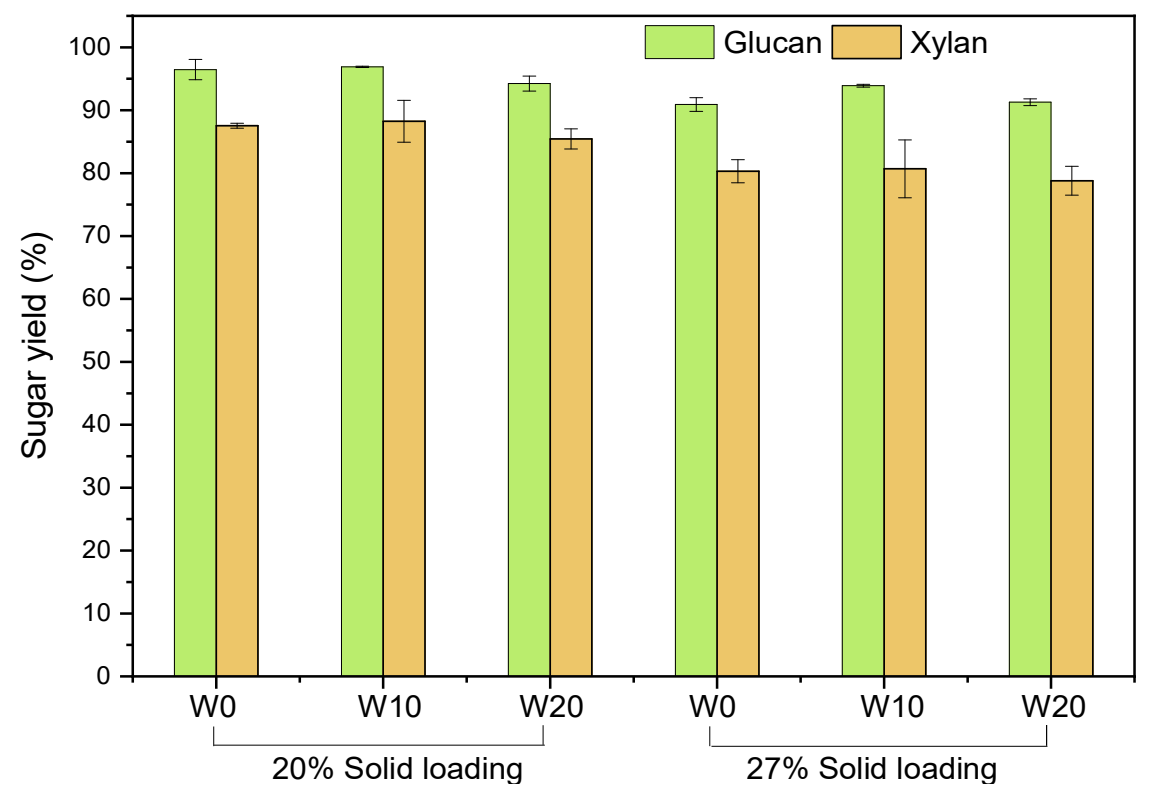

Figure 3.8 Switchgrass digestibility in response to $\mathrm{ChCl}$ :EG pretreatment under acidic condition with varying water addition and solid loadings.

Solvent recycling after pretreatment would improve not only waste management but also reduce chemical consumption and enrich soluble compounds. Therefore, it is worth evaluating the recyclability and reusability of $\mathrm{ChCl}$ :EG. It has been suggested that impurities (e.g., lignin derived phenolics, degraded carbohydrates) generated during pretreatment are a major factor affecting DES pretreatment performance (Kim et al., 2018). To test the robustness of $\mathrm{ChCl}: \mathrm{EG}$, a solid loading of $27 \%$, which would generate a high level of impurities, was selected for solvent recycling test. Acidified, aqueous $\mathrm{ChCl}$ : EG containing $10 \%$ water was selected as it gave similar lignin and xylan removal to neat $\mathrm{ChCl}$ :EG but was better than the one with $20 \%$ water (Figure 3.6). Xylan removal remained almost unchanged throughout all the pretreatment cycles, reaching about 70\% (Figure 3.9). Lignin removal was about $70 \%$ for the first three cycles but decreased to $60 \%$ in the fourth cycle. Glucan removal showed an increase from $2.5 \%$ to $6 \%$ from the first cycle to the 
third one, and the resultant residue showed a decrease in glucan content from $68 \%$ to $62 \%$ (Fig.3.10). In terms of digestibility, about $90 \%$ glucose yield was obtained during all the cycles, while the xylose yield was approximately $80 \%$ from the first to third cycle and decreased to $70 \%$ after the fourth cycle (Figure 3.11). Overall, aqueous $\mathrm{ChCl}$ :EG was successfully recycled and reused for at least three more cycles with similar performance. Upon the accumulation of impurities (e.g., lignin-derived compounds, soluble sugars) over the increased cycles, conditioning the recycled solvents would be necessary in order to improve its reusability. Lignin-derived compounds can be removed from the recycled solvent via adsorptive resin and membrane filtration (Narron et al., 2017). Soluble sugars can be removed via in situ upgrading into platform chemicals such as furfural as reported in the latter section.

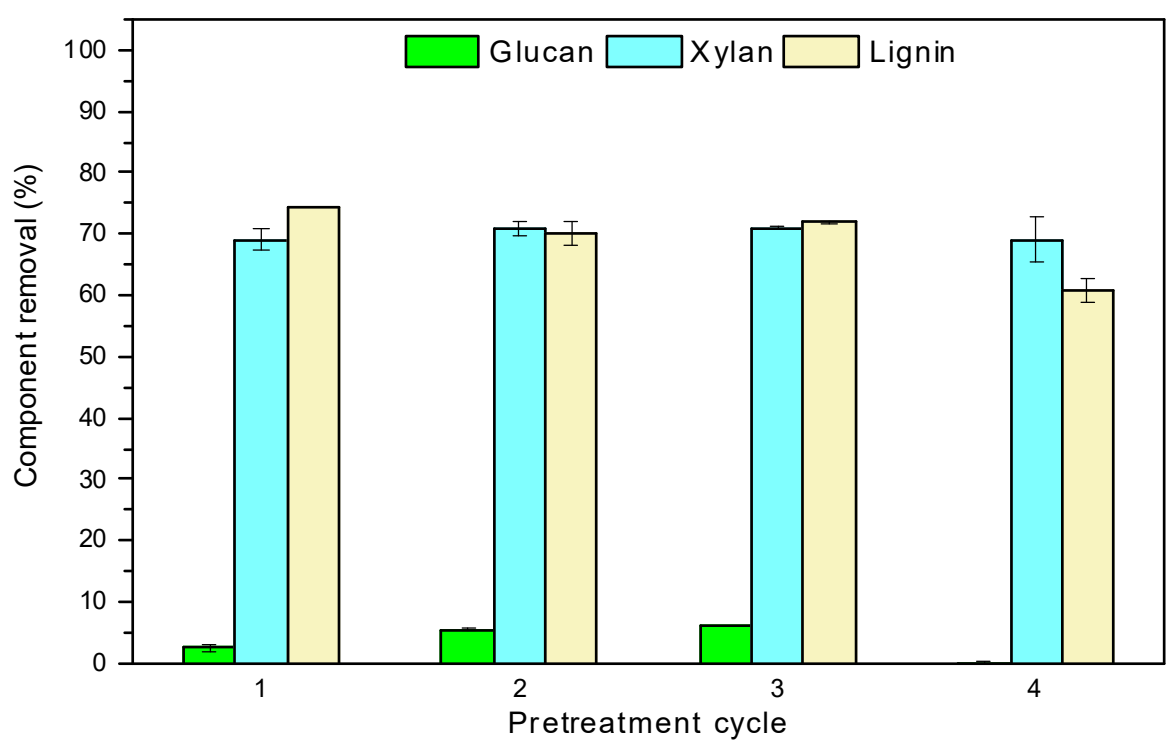

Figure 3.9 Switchgrass component removal in response to different pretreatment cycles under acidic condition using aqueous $\mathrm{ChCl}$ :EG with $10 \%$ water and at $27 \%$ solid loading. 


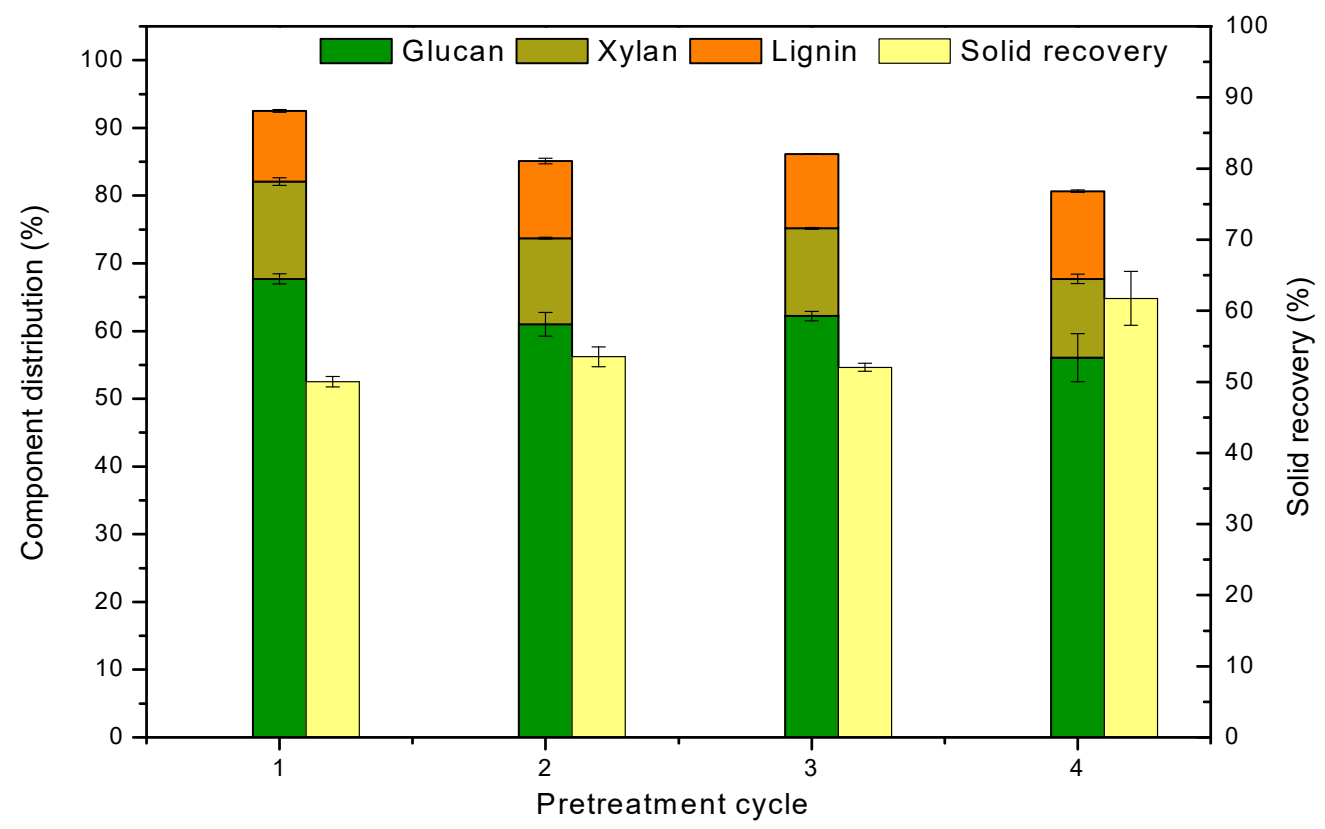

Figure 3.10 Switchgrass component distribution in response to different pretreatment cycles under acidic condition using aqueous $\mathrm{ChCl}$ :EG with $10 \%$ water and at $27 \%$ solid loading.

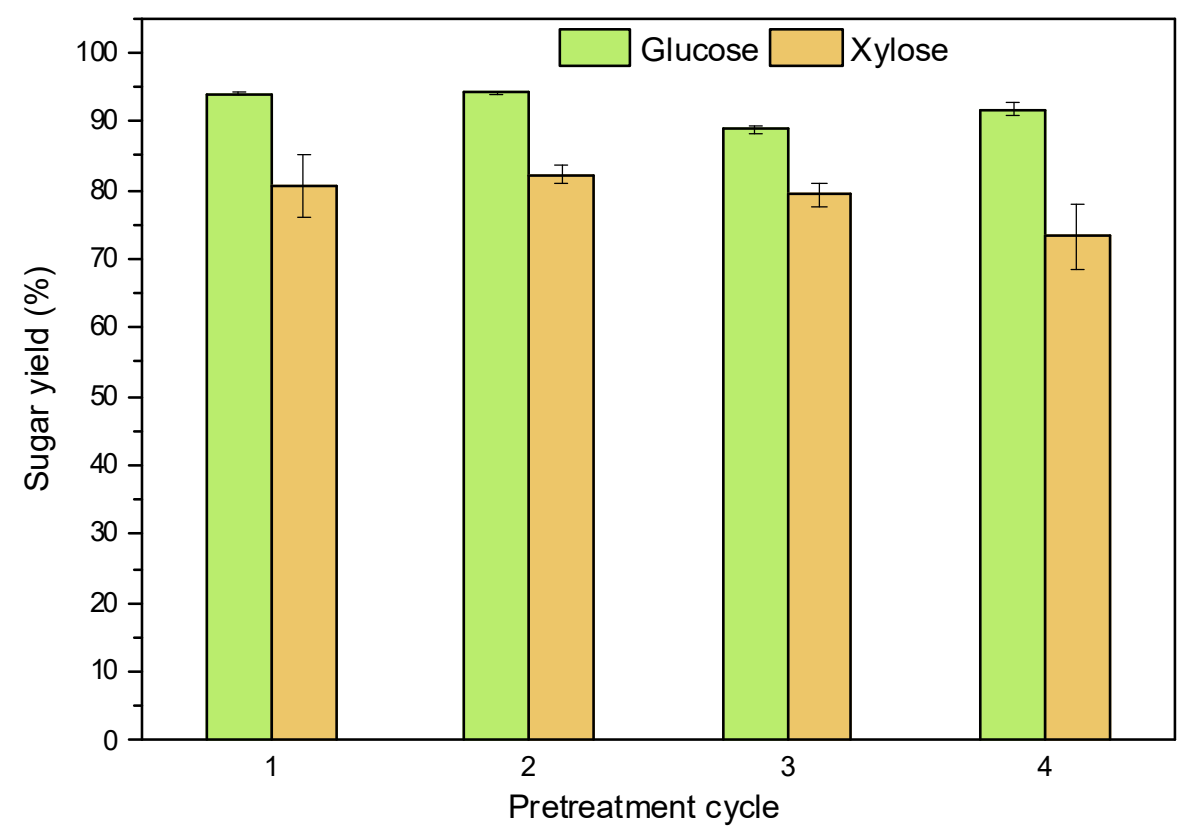

Figure 3.11 Switchgrass digestibility in response to different pretreatment cycles under acidic condition using aqueous $\mathrm{ChCl}: \mathrm{EG}$ with $10 \%$ water and at $27 \%$ solid loading.

\subsubsection{High solid loading enzymatic hydrolysis}

Concentrated sugar hydrolysate production without additional concentration, 
conditioning, or purification steps could realize high titers of cellulosic fuels, reducing distillation capital, and operating costs (Chen et al., 2016). Therefore, high solid loading enzymatic hydrolysis $(20 \%$ and $25 \%)$ was conducted to evaluate the feasibility of producing hydrolysate with highly concentrated monomeric sugars. Switchgrass pretreated at $20 \%$ and $27 \%$ solid loading using acidified, aqueous ChCl:EG (10\% water) was used for evaluation. Switchgrass pretreated at $20 \%$ solid loading gave a glucose and xylose yield of $85.2 \%$ and $75.3 \%$, respectively, after only $48 \mathrm{~h}$ of enzymatic hydrolysis, and the resulting hydrolysate contained $164.4 \mathrm{~g} / \mathrm{L}$ of glucose and $25.5 \mathrm{~g} / \mathrm{L}$ of xylose (Figure 3.12). When the solid loading of enzymatic hydrolysis was increased to $25 \%$ solid loading, the same pulp (pretreated at $20 \%$ ) even gave a $1 \%$ higher sugar yields $(86.2 \%$ glucose yield and $76.8 \%$ xylose), releasing $241.2 \mathrm{~g} / \mathrm{L}$ fermentable sugars (206.5 g/L glucose and $34.7 \mathrm{~g} / \mathrm{L}$ xylose). The sugar yields were lower when switchgrass pretreated at $27 \%$ solid loading was enzymatically hydrolyzed. However, the glucose yield of $82 \%$ and xylose yield of $69.9 \%$ were still obtainable at $20 \%$ solid loading enzymatic hydrolysis, which corresponded to a glucose and xylose concentration of 146.5 and $26.8 \mathrm{~g} / \mathrm{L}$, respectively (Figure 3.12). When the enzymatic hydrolysis was conducted at a $25 \%$ solid loading, the total fermentable sugars reached $190 \mathrm{~g} / \mathrm{L}(159.5 \mathrm{~g} / \mathrm{L}$ glucose and $31.4 \mathrm{~g} / \mathrm{L}$ xylose. Overall, both pulp samples were suitable for concentrated hydrolysis production while the one resulting from $20 \%$ solid loading pretreatment was more digestible at high-solid loading enzymatic hydrolysis. More concentrated sugar production would be expected upon enzymatic hydrolysis optimization. 


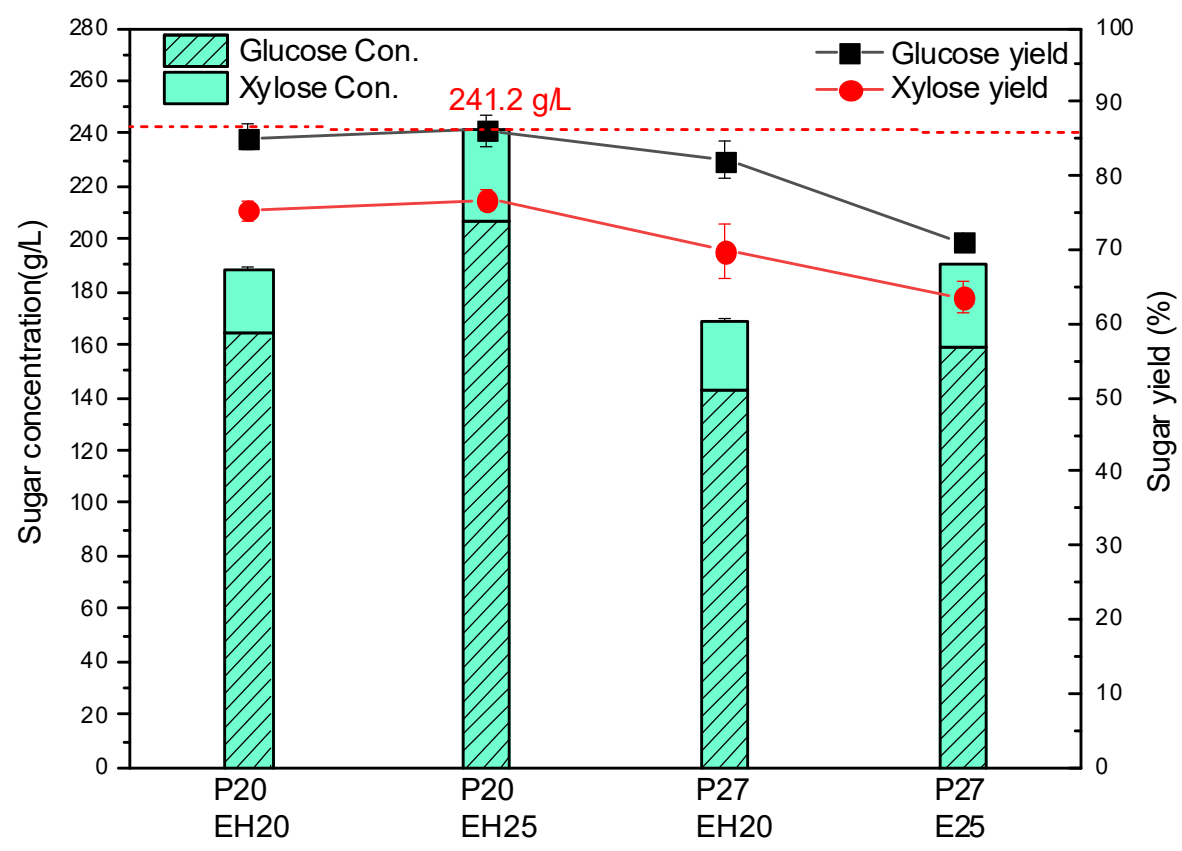

Figure 3.12 High-solid loading enzymatic hydrolysis of switchgrass pretreated at high solids using acidified, aqueous ChCl:EG with $10 \%$ water.

Some recently developed novel pretreatments, such as DMR (deacetylation and mechanical refining) and $\gamma$-irradiation pretreatment, were also reported to enable concentrated sugar hydrolysate production from lignocellulosic biomass (Chen et al., 2016; Zhou et al., 2016a). At solid loadings of 28\% and 25\%, DMR-pretreated corn stover and $\gamma$-irradiation-pretreated reed stover gave 230 and $231 \mathrm{~g} / \mathrm{L}$ monomeric sugar concentrations, respectively (Chen et al., 2016; Zhou et al., 2016a). With similar solid loadings for enzymatic hydrolysis the pulp resulting from our proposed DES pretreatment was able to release comparable or even larger amounts of total monomeric sugars $(241.2 \mathrm{~g} / \mathrm{L}$ corresponding to a glucose yield of $86.2 \%)$. Such impressive saccharification results indicated that aqueous $\mathrm{ChCl}$ :EG pretreatment developed in this study is a highly promising pretreatment technology to enable concentrated sugar production, which would be of great significance to biorefinery industry. 


\subsubsection{2,3-BD production from concentrated sugar hydrolysate}

Concentrated sugar hydrolysate directly resulting from high solid loading enzymatic hydrolysis would be a suitable substrate for high titer biofuel or chemical production. We tested two hydrolysates as presented in Figure 3.12 for 2,3-BD production via batch fermentation: $\mathrm{P} 20 / \mathrm{EH} 20$ and $\mathrm{P} 20 / \mathrm{EH} 25$. The initial total sugar concentrations in the fermentation media were adjusted to $174.5 \mathrm{~g} / \mathrm{L}$ (with $152.0 \mathrm{~g} / \mathrm{L}$ glucose and 22.5 g/L xylose) and 226.3 g/L (with 193.7g/L glucose and $32.6 \mathrm{~g} / \mathrm{L}$ xylose), respectively, since adding the stock solution of nutrients and $\mathrm{pH}$ adjustment slightly diluted the hydrolysate. For the initial sugar (glucose and xylose) concentration of $174.5 \mathrm{~g} / \mathrm{L}$, glucose was depleted within $36 \mathrm{~h}$ by $B$. vallismortis (NRRL B-14891), and 68.50 g/L 2,3-BD was produced, corresponding to a productivity of $1.90 \mathrm{~g} / \mathrm{L}-\mathrm{h}$ and a yield of $88.5 \%$ (Figure 3.13 ). In contrast, xylose was almost not converted until depletion of glucose, implying carbon catabolite repression in 2,3-BD fermentation by B. vallismortis. When the higher initial sugar concentration $(226.3 \mathrm{~g} / \mathrm{L})$ was tested, glucose was completely depleted along with gradual consumption of xylose (only $18.4 \%$ consumed in total) within 72 fermentation, resulting in a 2,3-BD titer of $90.2 \mathrm{~g} / \mathrm{L}$ with a productivity of $1.25 \mathrm{~g} / \mathrm{L}-\mathrm{h}$ and a yield of $89.6 \%$ (based on total sugar consumption) (Figure 3.14). The results suggested that $B$. vallismortis could not utilize xylose well in mixed C5 and C6 sugars toward 2,3-BD production. Nevertheless, compared with other studies, the titer, yield and productivity obtained in this study were the highest (Table 3.1). If only considering glucose consumption, the yield of 2,3-BD was as high as $93.0 \%$. Based on mass balance analysis, $152 \mathrm{~g}$ of 2,3-BD can be produced per $1000 \mathrm{~g}$ raw switchgrass (Figure 3.15). 


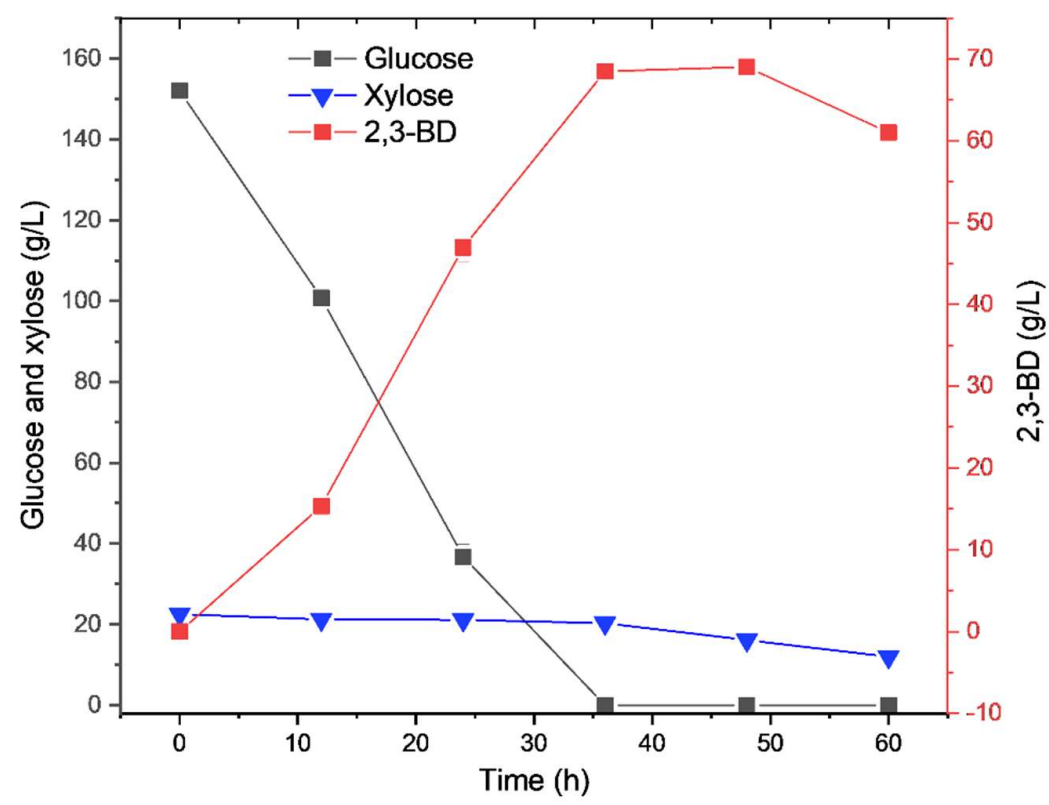

Figure 3.13 Fermentative production of 2,3-BD from switchgrass hydrolysate of $\mathrm{P} 20 / \mathrm{EH} 20$.

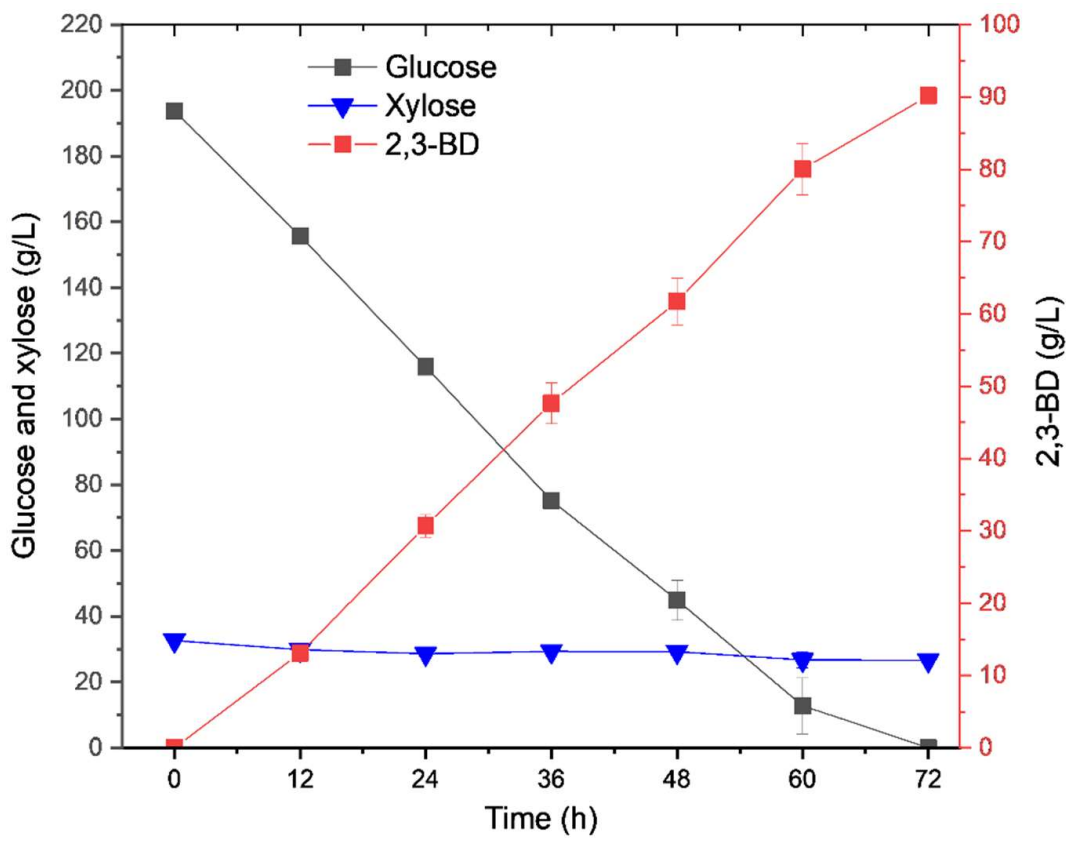

Figure 3.14 Fermentative production of 2,3-BD from switchgrass hydrolysate of $\mathrm{P} 20 / \mathrm{EH} 25$. 
Table 3.1 Summary of 2,3-BD production from lignocellulosic biomass.

\begin{tabular}{llcccl}
\hline Strain & Substrate & $\begin{array}{c}2,3-\mathrm{BD} \text { titer } \\
(\mathrm{g} / \mathrm{L})\end{array}$ & $\begin{array}{c}2,3-\mathrm{BD} \\
\text { yield }(\%)\end{array}$ & $\begin{array}{c}\text { 2,3-BD } \\
\text { productivity (g/L } \\
\mathrm{h}^{-1} \text { ) }\end{array}$ & References \\
\hline Klebsiella pneumoniae & Rice straw & 11.44 & 76.2 & 0.476 & (Saratale et al., 2016) \\
Enterobacter aerogenes & Sugarcane bagasse & 21 & 79.0 & 0.291 & (Um et al., 2017) \\
Klebsiella pneumoniae & Kenaf core powder & 10.42 & 77.0 & 0.434 & (Saratale et al., 2018) \\
Klebsiella oxytoca & Corn stover & 14.85 & 66.0 & 1 & (Guragain et al., 2016) \\
Enterobacter cloacae & Corn cob & 52.5 & 84.0 & 0.88 & (Ling et al., 2017) \\
Enterobacter aerogenes & yellow poplar & 14.72 & 79.5 & 1.19 & (Joo et al., 2016) \\
Paenibacillus polymyxa & Corn stover & 18.80 & 62.6 & 1.13 & (Ma et al., 2018) \\
Paenibacillus polymyxa & Wheat straw & 22 & 66.0 & 0.261 & (Adlakha \& Yazdani, \\
Bacillus Vallismortis & Switchgrass & 90.2 & 89.6 & 1.25 & This work \\
\hline
\end{tabular}




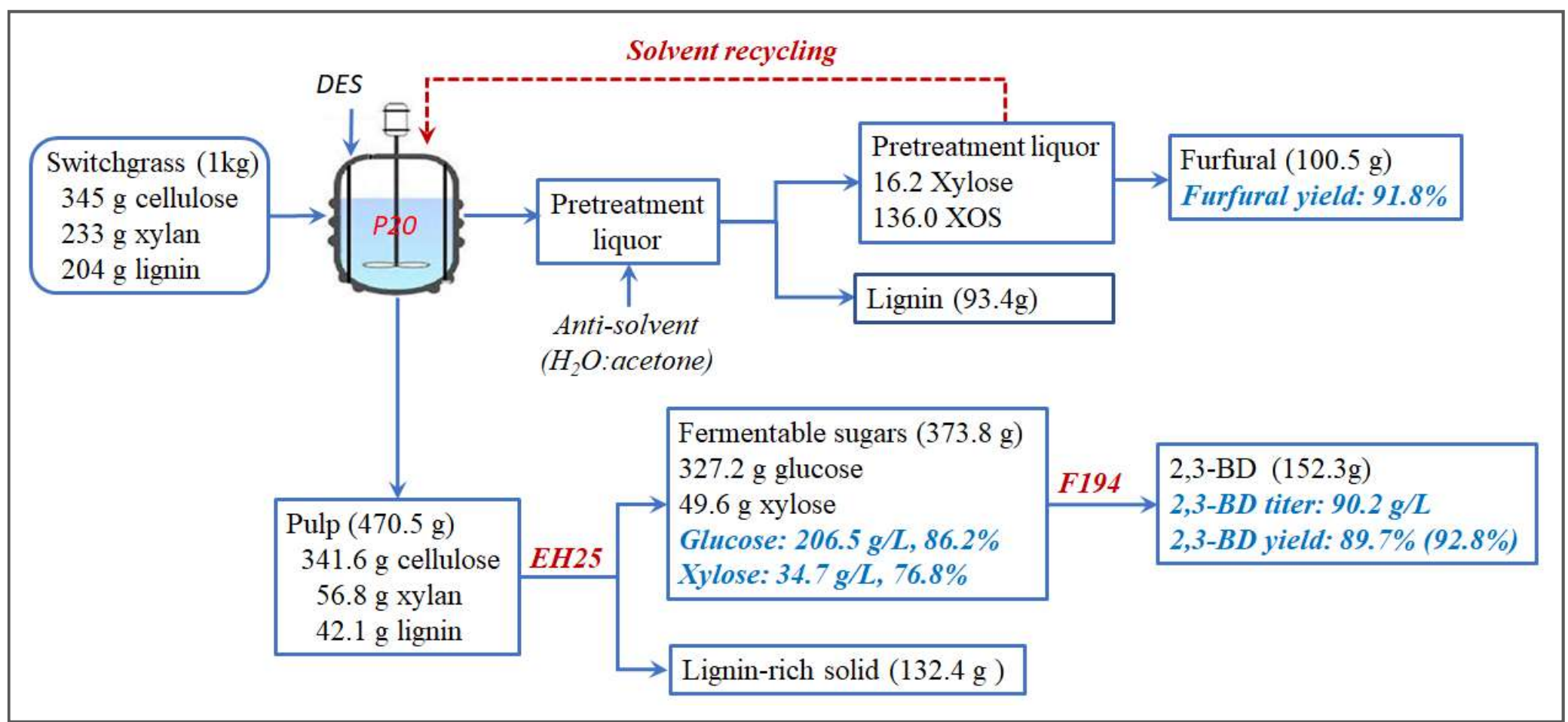

Figure 3.15 Mass flow analysis of the proposed biorefinery based on high-solid loading DES pretreatment for lignocellulose conversion into platform chemicals. 
Research efforts in strain development have improved the abilities of 2,3-BD producing strains for the utilization of glucose and xylose (Ji et al., 2011). However, the challenges with direct production of concentrated lignocellulosic hydrolysate largely limits economically viable production of 2,3-BD from lignocellulosic biomass. Due to low to moderate sugar concentrations in lignocellulosic hydrolysate, 2,3-BD titer ranged from about $10 \mathrm{~g} / \mathrm{L}$ to a maximum of $55.7 \mathrm{~g} / \mathrm{L}$ in previous studies (Adlakha \& Yazdani, 2015; Ling et al., 2017; Saratale et al., 2018). To the best of our knowledge, we reported for the first time more industrially relevant 2,3-BD production with the highest 2,3-BD TYP based on lignocellulosic biomass hydrolysate without additional concentration and detoxification. In the future study we will test more concentrated hydrolysates, different fermentation modes (e.g., fedbatch), and xylose-utilizing strains, to further improve 2,3-BD TPY toward commercial level production.

\subsubsection{Lignin fate}

As mentioned previously, acidified $\mathrm{ChCl}: \mathrm{EG}$ pretreatment rendered significant removal of xylan and lignin and generated a highly digestible celluloserich pulp (Figure 3.7). To understand the mechanism of the pretreatment and lignin properties, lignin purity and chemistry were examined. Compared with lignin generated from $20 \%$ solid loading pretreatment, lignin from $27 \%$ solid loading showed a lower purity. At 20\% solid loading, when water content in acidified $\mathrm{ChCl}$ :EG increased from $0 \%$ to $20 \%$, lignin purity increased from $83 \%$ to $86 \%$ (Table 1). A similar trend was also observed with $27 \%$ solid loading pretreatment, suggesting a positive effect of water addition on the lignin purity. A previous study suggested that EG can react with lignin by forming esters and ketals, and the reaction is influenced by both acid concentration and water content (Moghaddam et al., 2014). Plausibly, 
under higher water content (e.g., 20\%), EG was less reactive with lignin. This was supported by the compositional analysis results, which revealed that the lignin obtained from aqueous $\mathrm{ChCl}$ :EG containing 20\% water had the lowest EG content, and therefore the highest purity (Table 1 ). In addition, $\mathrm{ChCl}$ can also react with lignin(Alvarez-Vasco et al., 2016), and a higher water content may lower the reaction rate due to the interaction of $\mathrm{ChCl}$ with water. Overall, water influences the lignin purity by affecting the reaction between $\mathrm{ChCl}$ :EG and extracted lignin. In terms of lignin yield, more than $50 \%$ of solubilized lignin could be recovered at $20 \%$ solid loading using either neat or acidified, aqueous $\mathrm{ChCl:EG.} \mathrm{As} \mathrm{reported} \mathrm{by} \mathrm{a} \mathrm{previous}$ study, the aqueous DESs can dissolve lignin, and therefore, the lignin extracted into ChCl:EG could not be completely recovered via precipitation (Soares et al., 2017). Increasing the amount of water to enhance lignin precipitation or using membrane filtration may help improve the lignin recovery.(Kim et al., 2018). Increasing solid loading to $27 \%$ led to decreased lignin removal and recovery but had minor effects on lignin purity (Table 3.1). With solvent recycling, lignin purity remained at $80-82 \%$ while cumulative lignin yield was increased from $52 \%$ to $79 \%$ (Table 3.2 ). It is worthy of investigating the mechanism for increased lignin yield and possible formation of pseudo-lignin upon solvent recycling in the future study. 
Table 3.2 Lignin removal and recovery in response to high-solid loading DES pretreatment with a varying water content* .

\begin{tabular}{llllll}
\hline $\begin{array}{l}\text { Solid loading } \\
\text { of } \\
\text { pretreatment }\end{array}$ & $\begin{array}{l}\text { Water } \\
\text { content in } \\
\text { ChCl:EG }\end{array}$ & $\begin{array}{l}\text { Lignin } \\
\text { removal (\%) }\end{array}$ & $\begin{array}{l}\text { Lignin } \\
\text { yield (\%) }\end{array}$ & $\begin{array}{l}\text { EG } \\
(\%)\end{array}$ & $\begin{array}{l}\text { Lignin } \\
\text { purity (\%) }\end{array}$ \\
\hline $20 \%$ & W0 & $78.90 \pm 2.10$ & $53.23 \pm 2.15$ & $6.59 \pm 0.30$ & $83.34 \pm 0.28$ \\
& W10 & $79.39 \pm 0.15$ & $57.63 \pm 2.61$ & $6.39 \pm 0.30$ & $85.85 \pm 0.82$ \\
& W20 & $79.45 \pm 0.44$ & $52.98 \pm 2.31$ & $5.59 \pm 0.28$ & $86.14 \pm 0.32$ \\
\hline $27 \%$ & W0 & $74.55 \pm 0.77$ & $44.95 \pm 1.30$ & $6.26 \pm 0.24$ & $82.40 \pm 0.55$ \\
& W10 & $74.41 \pm 0.12$ & $51.77 \pm 2.44$ & $6.24 \pm 0.14$ & $83.16 \pm 0.15$ \\
& W20 & $69.41 \pm 1.29$ & $49.76 \pm 1.24$ & $5.72 \pm 0.13$ & $84.96 \pm 0.37$ \\
\hline
\end{tabular}

${ }^{*} \mathrm{ChCl}$ :EG pretreatment under acidic condition

${ }^{a} \mathrm{~W} 0, \mathrm{~W} 10$, and $\mathrm{W} 20$ stands for water addition at $0 \%, 10 \%$, and $10 \%$, respectively.

Table 3.3 Lignin removal and recovery in response to pretreatment solvent recycling*.

\begin{tabular}{llll}
\hline $\begin{array}{l}\text { Pretreatment } \\
\text { cycle }\end{array}$ & $\begin{array}{l}\text { Lignin } \\
\text { removal (\%) }\end{array}$ & $\begin{array}{l}\text { Cumulative } \\
\text { lignin yield (\%) }\end{array}$ & $\begin{array}{l}\text { Lignin } \\
\text { purity (\%) }\end{array}$ \\
\hline 1 & $74.41 \pm 0.12$ & $51.77 \pm 2.44$ & $83.16 \pm 0.15$ \\
2 & $70.10 \pm 1.83$ & $63.71 \pm 2.89$ & $82.10 \pm 0.43$ \\
3 & $72.00 \pm 0.25$ & $66.34 \pm 1.84$ & $82.65 \pm 0.53$ \\
4 & $60.89 \pm 1.83$ & $79.24 \pm 3.40$ & $80.36 \pm 0.39$ \\
\hline
\end{tabular}

* $27 \%$ solid pretreatment using acidified, aqueous $\mathrm{ChCl}: \mathrm{EG}$ with $10 \%$ water.

${ }^{a}$ Cumulative lignin yield calculated based on cumulative pretreatment cycles.

To understand the change in lignin chemistry, lignin from $20 \%$ and $27 \%$ solid loading pretreatment using acidified, aqueous $\mathrm{ChCl}$ :EG containing $10 \%$ water, denoted as L20 and L27, respectively, were examined using 2D HSQC NMR. Main ${ }^{13} \mathrm{C}-{ }^{1} \mathrm{H}$ cross-signals in the HSQC spectra of switchgrass lignin were assigned according to previous studies (Figure 3.16) (Samuel et al., 2011; Samuel et al., 2010). The presence of $\beta-\mathrm{O}-4$ substructure $(\mathrm{A})$ in native lignin was detected by the cross 
peaks at $\delta \mathrm{C} / \delta \mathrm{H} 71.84 / 4.86\left(\mathrm{~A}_{\alpha}\right), 83.68 / 4.29\left(\mathrm{~A}_{\beta}\right)$ and 60.00/3.60 $\left(\mathrm{A}_{\gamma}\right)$. However, in L20 and L27, the contours for $\beta-O-4$ linkages shrank. Semi-quantification revealed the percentages of $\beta-\mathrm{O}-4$ linkage in L20 and L27 were $1.53 \%$ and $4.98 \%$, respectively, as compared to $46.72 \%$ in NL (Table 3.3). Acidified ChCl:EG may work similarly as $\mathrm{ChCl}$ :lactic acid (LA), which cleaves $\beta-\mathrm{O}-4$ in lignin to facilitate lignin solubilization (Alvarez-Vasco et al., 2016). Taken together, these results indicated the important role of acidic proton in DES pretreatment. Previous studies suggested that the preservation of $\beta-\mathrm{O}-4$ linkages is critical for monomer production via catalytic depolymerization (Renders et al., 2017). Therefore, L20 may not be suitable for the production of aromatics via depolymerization. However, the elimination of ether bonds may render lignin with better polymer stability. Taking into account its relatively high purity, such lignin may serve as good building blocks for the synthesis of advanced materials. Differing from L20, L27 preserved some of the ether bonds during pretreatment (Figure 3.16 \&Table 3.3), implying that by adjusting the pretreatment conditions (e.g., solid loading, pretreatment time) during acidified ChCl:EG pretreatment, lignin with different structures (e.g., varying molecular weights, different abundance of $\beta$-O-4 linkages) can be obtained.

The dominant peaks shown in aromatic regions were syringyl (S), guaiacyl (G), $p$-coumarate ( $p$-CA), and ferulate (FA). The apparent difference in the aromatic region between native lignin and DES lignin was the decreased peak intensity for FA and $p$-CA in L20 and L27. As shown in Table 3.3, the relative percentages of FA in L20 and L27 were 4.98 and 5.46\%, respectively, while 9.35\% in native lignin. Similarly, the relative percentages of $p$-CA in DES lignin were also lower than that in native lignin. In grass lignin, both $p$-CA and FA acylate arabinoxylans, and serve as the bridge between lignin and polysaccharide via formation of ether and ester linkages 
(Kim \& Ralph, 2010). The decreased relative percentage of $p$-CA and FA suggested the cleavage of lignin-polysaccharide during pretreatment, which facilitated the isolation of lignin and xylan from switchgrass.

Table 3.4 Semi-quantification of the $\beta$-O-4 and hydroxycinnamates abundance in lignin.

\begin{tabular}{llll}
\hline Lignin & $\beta-\mathrm{O}-4$ & $p$-CA & FA \\
\hline Native lignin & 46.72 & 33.41 & 9.35 \\
L20 & 1.53 & 29.96 & 4.98 \\
L27 & 4.98 & 30.24 & 5.46 \\
\hline
\end{tabular}




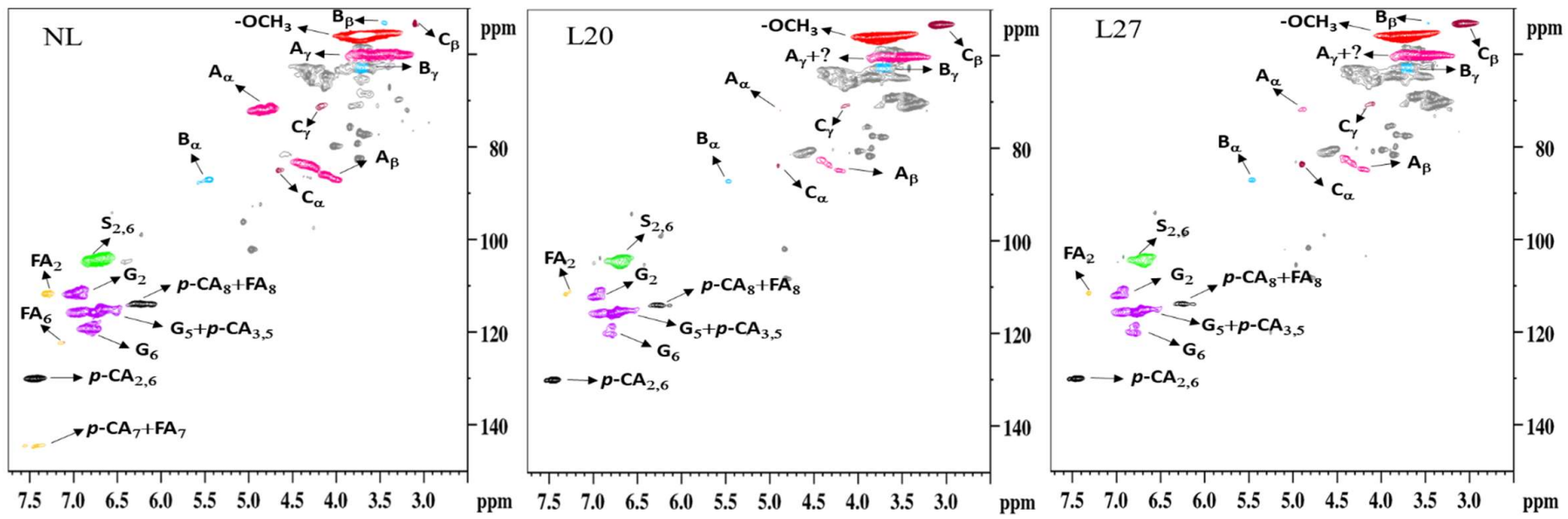

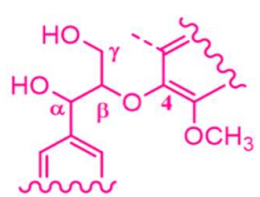

$\mathrm{A}(\beta-\mathrm{O}-4)$

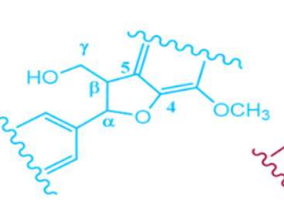

$\mathrm{B}(\beta-5)$

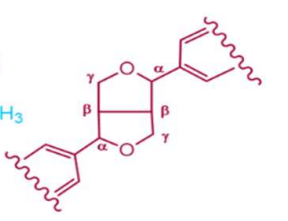

$C(\beta-\beta)$

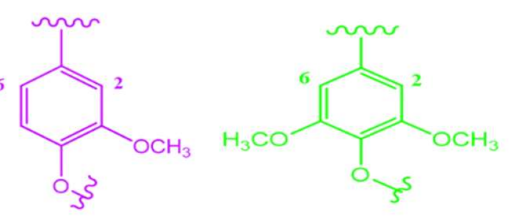

G(Guaiacyl unit)

S(Syringyl unit)
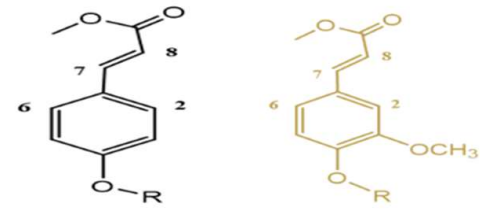

FA(Ferulate)

Figure 3.16 2D HSQC NMR analysis of DES lignin. 
The GPC results of L20 and L27 compared to that of native lignin are given in Table 3.4. The average molecular weight (Mw) of native lignin was $9544 \mathrm{~g} / \mathrm{mol}$, attributed to the macropolymer structure of native lignin. The Mw decreased in both L20 and L27, confirming that lignin partly depolymerizes during the $\mathrm{ChCl}: \mathrm{EG}$ pretreatment. The polydispersity (PD) also decreased with the $\mathrm{ChCl}$ :EG derived lignin for narrower molecular distribution. The average $\mathrm{Mw}$ and $\mathrm{PD}$ were both lower with L20 compared to L27 (3933 g/mol vs. $4600 \mathrm{~g} / \mathrm{mol}$, and 3.34 vs. 3.75), which imply that lignin decomposes more severely during the pretreatment with $20 \%$ solid loading. As previously shown in Figure 3.16, the ether bond cleavages were less significant in the case of $27 \%$ solid loading pretreatment, thus producing a less depolymerized lignin.

Table 3.5 Average molecular weight and polydispersity (PD) of DES lignin*.

\begin{tabular}{llll}
\hline Samples & $\mathrm{Mn}$ & $\mathrm{Mw}$ & $\mathrm{PD}$ \\
\hline Native lignin & 1951 & 9544.5 & 4.89 \\
L20 & 1176 & 3932.5 & 3.34 \\
L27 & 1229 & 4599.5 & 3.75 \\
\hline
\end{tabular}

* L20 and L27 stand for lignin recovered from $20 \%$ and $27 \%$ solid-loading pretreatment, respectively, under acidic condition using aqueous $\mathrm{ChCl}$ : $\mathrm{EG}$ with $10 \%$ water addition.

Thermal stability of lignin was determined based on the TGA results shown in Figure 3.17. Compared to native lignin, L20 and L27 both decomposed at higher temperatures. Since the weak aryl ether bonds were partly cleaved during $\mathrm{ChCl}: \mathrm{EG}$ pretreatment, the bonds remaining in the recovered lignin are thermally more stable. L20 had a higher thermal stability than L27 to produce a higher amount of solid residue upon pyrolysis despite its lower $\mathrm{Mw}$ and $\mathrm{PD}$. In addition to ether bonds cleavage, secondary reactions likely occurred during the DES pretreatment. The reactive free radicals generated from the primary depolymerization of lignin could couple each other to form a new molecular structure with thermally more stable bonds (e.g., intra aromatic $\mathrm{C}-\mathrm{C}$ bonds). Since lignin is depolymerized more severely with a 
lower solid loading pretreatment, it is expected that secondary reactions during the pretreatment were also more pronounced with L20. In comparison, L27 better preserved aryl ether bonds and some other weak bonds, and thus had higher volatility. Higher volatility is usually desired in order to upgrade the lignin for biofuels and chemicals. On the other hand, higher thermal stability and narrower Mw distribution are preferred if the lignin is to be used as biocarbon materials, such as carbon fiber.

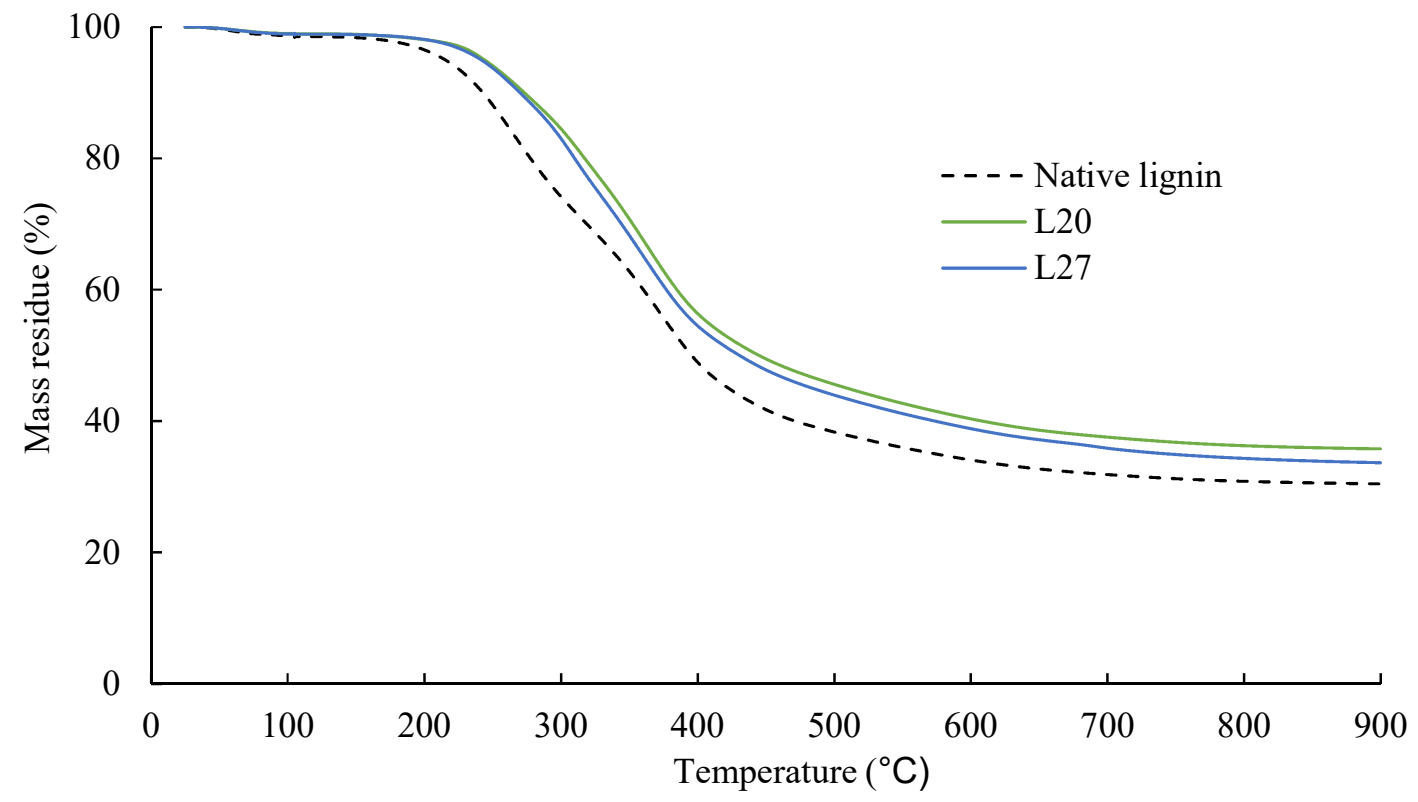

Figure 3.17 TGA profiles of DES lignin.

Lignin is known as an important source of renewable aromatics. For instance, lignin-derived phenolic monomers are valuable chemicals that have wide applications. Thus, $\mathrm{ChCl}$ :EG lignin were also pyrolyzed for phenolic monomers and the results were compared with that from native lignin (Table 3.5). Due to herbaceous nature of switchgrass and demethoxylation during pyrolysis, $\mathrm{H}$ type phenols were most abundant among the monomers, followed by G type and $\mathrm{S}$ type of monomers at all three kinds of lignin. Also, 4-vinylphenol and 4-vinyl guaiacol were the two major monomers, which were mainly produced from decomposition of hydroxycinnamate groups and $\beta-\mathrm{O}-4$ cleavages (Zhou et al., 2016b). Pyrolysis of native lignin delivered 
the highest total monomer yield, which was $14.58 \%$. In comparison, the yields were 8.03\% from L20 and $9.08 \%$ from L27 due to the decreased contents of ether linkages and increased thermal resistivity of $\mathrm{ChCl}$ :EG lignin for volatilization. However, simpler and short-side chain phenols, such as phenol, guaiacol, cresol, methyguaiacol and ethylphenol, were more selectively produced from L20 and L27 compared to native lignin since the aromatic side chains were partially cleaved during the $\mathrm{ChCl:EG}$ pretreatment. L27 produced more monomers than L20, which well corresponded to the less modified lignin structure and higher volatility of L27 compared to L20. Especially, higher yield of 4-vinylphenol was obtained with L27 because it contained more $\beta-\mathrm{O}-4$ linkages. 
Table 3.6 Yields of phenolic monomers produced from lignin pyrolysis.

\begin{tabular}{lccc}
\hline Yield (\%) & Native lignin & L20 $^{\text {a }}$ & L27 $^{\text {a }}$ \\
\hline Phenol & 0.19 & 0.24 & 0.23 \\
Guaiacol & 0.33 & 0.61 & 0.63 \\
o-Cresol & 0.06 & 0.37 & 0.39 \\
p-Cresol & 0.21 & 0.79 & 0.86 \\
p-Methylguaiacol & 0.40 & 0.92 & 0.91 \\
4-Ethylphenol & 0.05 & 0.21 & 0.21 \\
4-Ethylguaiacol & 0.33 & 0.14 & 0.14 \\
4-Vinyl Phenol & 5.03 & 2.35 & 2.91 \\
4-Vinyl Guaiacol & 5.26 & 0.77 & 0.91 \\
Eugenol & 0.03 & 0.06 & 0.08 \\
Syringol & 0.37 & 0.24 & 0.27 \\
Isoeugenol & 0.09 & 0.06 & 0.07 \\
trans-Isoeugenol & 0.25 & 0.28 & 0.35 \\
1,2,4-Trimethoxybenzene & 0.41 & 0.37 & 0.41 \\
Vanillin & 0.28 & 0.07 & 0.10 \\
Apocynin & 0.15 & 0.08 & 0.08 \\
3',5'-Dimethoxyacetophenone & 0.34 & 0.19 & 0.22 \\
2,6-Dimethoxy-4-allylphenol & 0.56 & 0.26 & 0.33 \\
Acetophenone, 4'-hydroxy-3',5'- & & & \\
dimethoxy- & 0.24 & 0.00 & 0.00 \\
Total & 14.58 & 8.03 & 9.08 \\
\hline
\end{tabular}

${ }^{\text {a }}$ L20 and L27 stand for lignin recovered from $20 \%$ and $27 \%$ solid-loading pretreatment, respectively, under acidic condition using aqueous $\mathrm{ChCl}$ : $\mathrm{EG}$ with $10 \%$ water addition. 
Summarizing the characterization results described above, it can be concluded that the $\mathrm{ChCl}$ :EG lignin is comparable to switchgrass lignin extracted otherwise (Samuel et al., 2011; Samuel et al., 2010), and higher solid loading pretreatment actually improved the quality of the resulting lignin in terms of volatilization and upgrading for phenolic monomers.

\subsubsection{Pretreatment liquor upgrading for furfural production}

As discussed above, $\mathrm{ChCl}$ :EG pretreatment can efficiently solubilize xylan under acidic condition, leading to up to $75.6 \%$ xylan removal at high-solid pretreatment. Without solvent recycling, xylan-based sugars in the liquor accounted for up to $94 \%$ xylan removed during the pretreatment, most of which was presented in a form of xylo-oligosaccharide (XOS) (Table 3.6). In contrast, solvent recycling led to $70.0 \%$ xylan removal from switchgrass, but only $56.8 \%$ xylan recovery in the pretreatment liquor. Reduced xylan recovery in the recycled liquor implied obvious degradation of xylan-based sugars upon solvent recycling while no furfural was detected in any pretreatment liquor. Nonetheless, solvent recycling had many benefits as aforementioned. One was highly enriched xylan-based sugars in the pretreatment liquor, reaching $78.7 \mathrm{~g} / \mathrm{L} \mathrm{XOS} \mathrm{and} 14.52 \mathrm{~g} / \mathrm{L}$ xylose, which can facilitate further upgrading of pretreatment liquor. 
Table 3.7 Xylan removal and recovery in pretreatment liquor for furfural production.

\begin{tabular}{|c|c|c|c|c|c|c|c|c|}
\hline${ }_{*}^{\text {Pretreatment }}$ & $\begin{array}{l}\text { Swtichgrass } \\
\text { / Xylan } \\
(\mathrm{g} / \mathrm{g})^{\mathrm{a}}\end{array}$ & $\begin{array}{l}\text { aDES } \\
(g)^{b}\end{array}$ & $\begin{array}{l}\text { Xylan } \\
\text { removal (\%) } \\
\text { c }\end{array}$ & $\begin{array}{l}\text { Xylan yield } \\
\text { in liquor }(\%) \\
\text { d }\end{array}$ & $\begin{array}{l}\text { Xylan } \\
\text { present as } \\
\text { xylose }(\%)^{\mathrm{e}}\end{array}$ & $\begin{array}{l}\text { Xylan } \\
\text { present as } \\
\text { XOS (\%) }\end{array}$ & $\begin{array}{l}\text { Furfural } \\
\text { yield }(\%) \\
\mathrm{f}\end{array}$ & $\begin{array}{l}\text { Furfural } \\
\text { production } \\
\text { (g) }\end{array}$ \\
\hline P20 (1 cycle) & $1000 / 233$ & 4000.0 & 75.6 & 85.3 & 8.1 & 77.2 & 91.8 & 100.5 \\
\hline P27 (1 cycle) & $1000 / 233$ & 2703.7 & 69.1 & 94.4 & 14.2 & 80.2 & 91.3 & 101.1 \\
\hline P27 (4 cyles) & $1000 / 233$ & 675.9 & 70.0 & 56.8 & 8.5 & 48.3 & 84.6 & 57.0 \\
\hline
\end{tabular}

${ }^{*}$ P20 and P27 stand for $20 \%$ and $27 \%$ solid loadings for pretreatment, respectively.

a Per $1000 \mathrm{~g}$ switchgrass contained 233 g xylan.

${ }^{\mathrm{b}}$ Amount of acidified, aqueous DES added per $1 \mathrm{~kg}$ switchgrass at different pretreatment modes.

${ }^{\mathrm{c}}$ Xylan removal on a basis of original $233 \mathrm{~g}$ xylan per kg switchgrass.

${ }^{\mathrm{d}}$ Xylan yield in liquor on a basis of the total amount of xylan removed/solubilized.

${ }^{\mathrm{e}}$ Solublized xylan presented in pretreatment liquor in the forms of xylose and xylo-oligosaccharides (XOS).

${ }^{\mathrm{f}}$ Furfural yield on a basis of total xylan-based sugars in pretreatment liquor. 
Since many ChCl-based DESs appear to be suitable reaction media for xylan/xylose to furfural, we further upgraded the pretreatment liquor to furfural by taking advantage of such a ChCl:EG-based liquor as both reaction medium and substrate (Zhang et al., 2014). This was also the first study to use $\mathrm{ChCl}$ :EG as a reaction medium for furfural production. Since $\mathrm{AlCl}_{3}$ has been shown to accelerate furfural production in ChCl-based DESs, its loading was first optimized using aqueous $\mathrm{ChCl}$ :EG (50 wt\%) containing $100 \mathrm{~g} / \mathrm{L}$ pure xylose. As shown in Figure 3.18, the furfural yield leveled off when $1.5(\mathrm{w} / \mathrm{v}) \% \mathrm{AlCl}_{3}$ was loaded, reaching $90 \%$ overall yield. Also in agreement with prior study (Zhang et al., 2014), water instead of DES as a reaction medium showed a $34 \%$ lower furfural yield, suggesting strong synergism between $\mathrm{ChCl}$ : $\mathrm{EG}$ and $\mathrm{AlCl}_{3}$ in converting xylose to furfural.

Applying $1.5 \% \mathrm{AlCl}_{3}$ to liquor upgrading, we found that similar overall furfural yields (91-92\%) were obtained with unrecycled liquor (Table 3.6). Recycled liquor gave a reduced, but still outstanding overall furfural yield (84.52\%). It was possible that repeatedly recycled liquor may contain more impurities, which in turn affected xylose conversion into furfural. Nonetheless, recycled liquor gave a high furfural titer since it was enriched with xylan-based sugars. Furfural was detected at $12.95 \mathrm{~g} / \mathrm{L}$ in MIBK and its partition coefficient for MIBK/aqueous was 2.8 (Figure 3.19). Correspondingly, the MIBK phase contained $92 \%$ of the produced furfural, and can be easily separated from aqueous phase due to its immiscibility with aqueous ChCl:EG (Di Marino et al., 2017). MIBK can be recovered from the organic phase via distillation since it has a relatively low boiling point. The aqueous phase, which mainly contained $\mathrm{ChCl}, \mathrm{EG}$, and water but no xylan-based sugars, can be recycled by removing water as needed. However, based on total xylan removal, solvent recycling gave a much lower furfural output than no solvent recycling (Table 3.6). On a basis of 
$1000 \mathrm{~g}$ raw switchgrass was $57.0 \mathrm{~g}$ furfural produced for solvent recycling vs. about $101 \mathrm{~g}$ furfural for no solvent recycling (Table 3.6 \&Figure 3.15). Therefore, research efforts should be invested in the future study to minimize xylan degradation upon solvent recycling and condition recycled liquor as needed for pretreatment liquor upgrading.

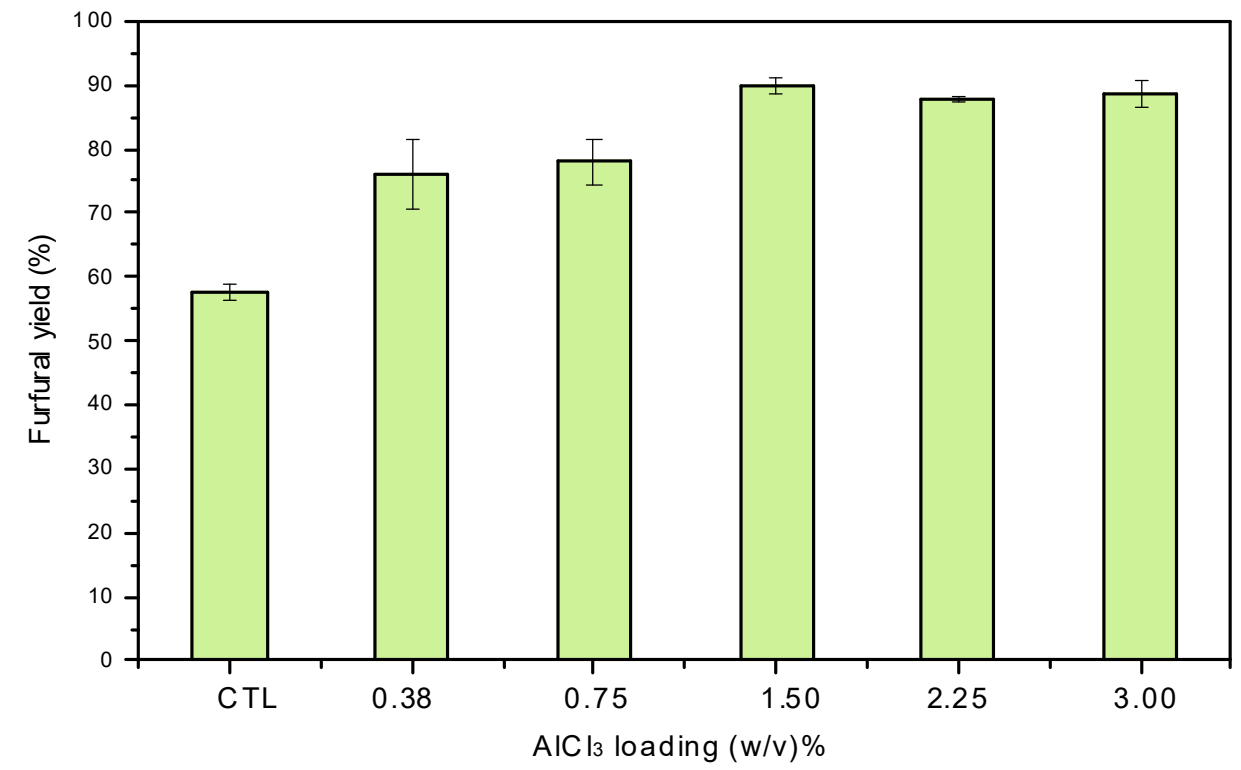

Figure 3.18 Effect of $\mathrm{AlCl} 3$ addition on $100 \mathrm{~g} / \mathrm{L}$ xylose conversion into furfural.

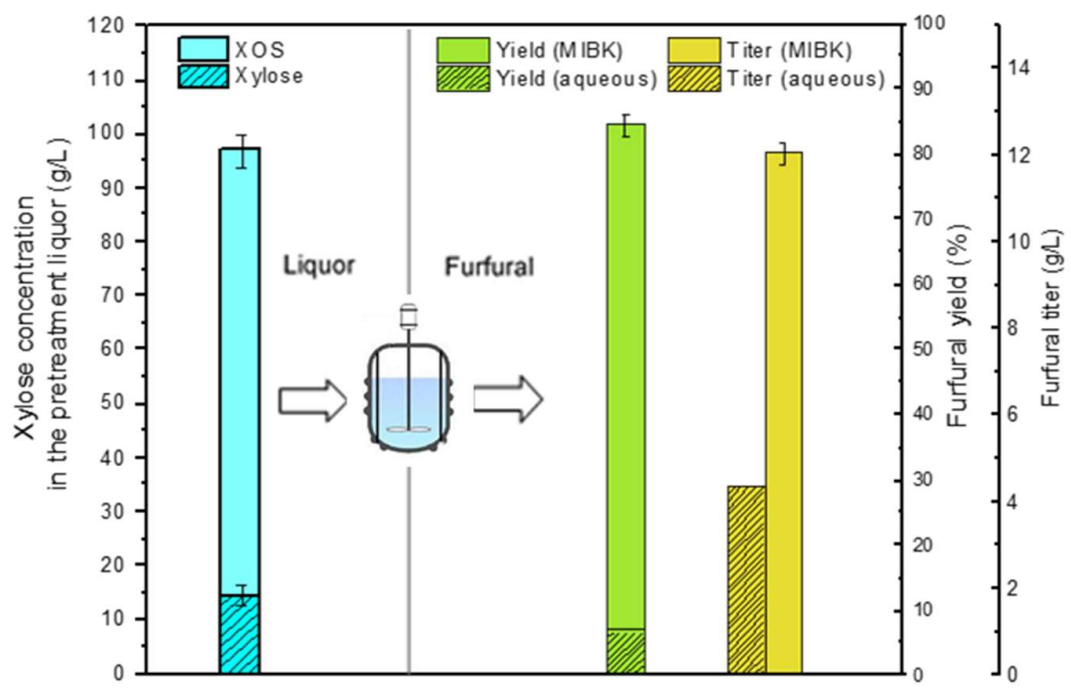

Figure 3.19 Furfural production from the pretreatment liquor. 


\subsection{Conclusion}

High solid loading, aqueous ChCl:EG-based pretreatments were highly efficient under acidic condition for lignin and xylan removal, rendering a celluloserich residue with high digestibility for concentrated sugar production. Within only 48 h of $25 \%$ solid-loading enzymatic hydrolysis, the pulp pretreated at $20 \%$ solids was hydrolyzed into $241.2 \mathrm{~g} / \mathrm{L}$ fermentable sugars (206.5 g/L glucose and $34.7 \mathrm{~g} / \mathrm{L}$ xylose) with a glucose yield over $85 \%$. Such hydrolysate allowed 2,3 -BD production with a titer as high as $90.2 \mathrm{~g} / \mathrm{L}$ without additional sugar concentration for the first time. The pulp pretreated at $27 \%$ solids can also be enzymatically hydrolyzed at high solids although a lesser amount of sugars was released. On the other hand, the lignin extracted at $27 \%$ solid loading pretreatment was less depolymerized, thus having better thermal volatility and producing a higher yield of phenolic monomers upon pyrolysis. $\mathrm{ChCl}$ :EG was successfully recycled and reused for at least three pretreatment cycles. Recycling $\mathrm{ChCl}$ :EG for pretreatment improved the accumulative lignin yield while not affecting the lignin purity. Solvent recycling also enriched xylan-derived sugars in the liquor, which can be used as both reaction medium and substrate for furfural production. This study developed a high solid loading pretreatment using high performance, recyclable aqueous $\mathrm{ChCl}$ :EG, which enabled industrially relevant production of sugar hydrolysates and platform chemicals. 


\section{Chapter 4 Ternary Deep Eutectic Solvents for Switchgrass Fractionation}

\subsection{Introduction}

Lignocellulosic biomass is a renewable, easily available, and promising feedstock for producing fuels and chemicals, and is considered a good replacement of fossil resources. It is mainly composed of cellulose, hemicellulose and lignin. Cellulose is a biopolymer with repeated glucose units linked via $\beta$-1,4-glycocidic bonds, and covered by hemicellulose and lignin. One common approach for producing fuels and chemicals is to release the monomeric sugars from polysaccharide, and convert the sugars into products via biological routes (Zhai et al., 2018). Due to the recalcitrant nature of lignocellulosic biomass, a pretreatment step is still necessary to remove hemicellulose and/or lignin from lignocellulosic biomass to facilitate efficient sugar conversion.

Various pretreatment, including acid, alkali, steam explosion, and ionic liquids (ILs), have been developed for achieving good polysaccharide conversion, with some successfully deployed at large scale (Mood et al., 2013; Mosier et al., 2005). ILs, a green solvent featuring low vapor pressure, thermal stability, and non-flammability, has attracted much attention in the past decade for lignocellulosic biomass fractionation. The effectiveness of ILs in pretreatment has been demonstrated by many studies (An et al., 2015; Hou et al., 2015; Socha et al., 2014), but its cost and environmental concerns over their toxicity and biodegradability are still a critical barrier for its large scale application (Xia et al., 2014). Deep eutectic solvents (DESs), an analogy to ILs, are considered to be a good alternative to ILs for pretreatment. In addition to the common green features of ILs, DESs also feature simpler and 
"greener" synthesis processes, lower raw material costs, and less sensitive to water and impurities (Gorke et al., 2008; Lynam et al., 2017; Zhao et al., 2018). Early work on DESs based pretreatment has revealed that certain DESs, such as choline chloride:formic acid (ChCl:FA), $\mathrm{ChCl}$ :glycerol(ChCl:Gly), and $\mathrm{ChCl}$ :ethanolamine(ChCl:EA), are effective in enhancing the digestibility of pretreated biomass, and the glucose yield over $90 \%$ was achievable (Chen $\&$ Wan, 2018; Xu et al., 2016; Zhao et al., 2018). The digestibility of pretreated biomass was comparable to those from most effective ILs-based pretreatment. Thus, DESs offers new opportunities for green solvents-based pretreatment at lower cost. However, to make DESs based-pretreatment an applicable method for lignocellulosic biomass fractionation at large scale, the overall processing costs need to be decreased. High solid loading ( $\geq 15 \%$ ) processing of lignocellulosic biomass in different operation units (e.g., pretreatment, enzymatic hydrolysis) is always pursued in different pretreatment as it reduces the energy input, waste generation, and chemical consumption (Modenbach \& Nokes, 2012). Although high solid loading processing provide many benefits, it remains to be challenging as mass and heat transfer is limited at high solid loading. As a result, most previous DESs based-pretreatment and enzymatic hydrolysis were limited to low to moderate solid loading $(\leq 10 \%)$. To solve the dilemma of decreasing costs associated with DESs pretreatment and compromised performance at high solid loading, new pretreatment processes and/or new DESs should be designed.

In addition to efficient utilization of carbohydrates from lignocellulosic biomass, recent years have witnessed an increased demand for the valorization of lignin, a product previously deemed as a waste in biorefinery. Indeed, the valorization of lignin into value-added products not only increases revenue from lignocellulosic 
biomass, but also reduces the reliance on single product output by expanding the spectrum of output products from biorefinery (Stoklosa et al., 2018). Various valorization routes have been proposed to convert lignin into valuable products, such as carbon fiber, microbial lipids, supercapicators, and aromatic monomers (Ho et al., 2018; Rinaldi et al., 2016). No matter which route it takes toward lignin upgrading, efficient solubilizing and recovering lignin is often the prerequisite. DESs are good solvents for dissolving lignin, and some DESs, such as $\mathrm{ChCl}$ :EG, were reported to dissolving more than $10 \%(\mathrm{w} / \mathrm{v})$ lignin at room temperature (Di Marino et al., 2016). However, the good capability of solubilizing lignin by such DESs does not always guarantee good delignification performance during pretreatment. Previous studies reported that $24 \mathrm{~h}$ pretreatment time is required for $\mathrm{ChCl}: \mathrm{EG}$ to achieve $88 \%$ delignification in 5\% solid loading pretreatment (Zhang et al., 2016a). Due to tunable properties of DESs, such DESs could be tailored/redesigned to meet different purposes of application (Tang et al., 2017). Given $\mathrm{ChCl}$ :EG is highly capable of dissolving lignin and is a well-studied DES with applications in many fields, it is possible to redesign this DES in an easy way to improve its performance in pretreatment. The fractionation of the major components in lignocellulosic biomass is a process of cleaving numerous bonds, such as ether/ester bonds and glycocidic bonds, which requires the participation of protons. This is supported by previous studies, which found that carboxylic acids based-DESs offered good delignification and hemicellulose removal (Chen \& Wan, 2018; Xu et al., 2016).Therefore, a strong proton donor in DESs may be critical for its good performance in pretreatment. The solid organic aromatic acid, $p$-toluenesulfonic acid (PSTA), is a good and strong proton donor (Chen et al., 2017). By incorporating PTSA into the ChCl:EG may 
provide a DES with greatly enhanced performance in fractionation of lignocellulosic biomass.

In this study, the ternary DES, namely $\mathrm{ChCl}$ :EG:PTSA, and its analogues were synthesized and screened by evaluating its performance in pretreatment of switchgrass, a representative lignocellulosic biomass. The ternary DESs with good performance were further used for pretreatment at 35\% solid loading at ambient pressure, and the resultant switchgrass were enzymatically digested at $20 \%(\mathrm{w} / \mathrm{w})$ solid loading for hydrolysate preparation.

\subsection{Materials and methods}

\subsubsection{Feedstock and chemicals}

Switchgrass was collected from the South Farm at University of Missouri in Columbia, Missouri, USA. Raw switchgrass was air-dried and ground through a 2 mm-mesh screen. Hydrolytic enzymes (Cellic ${ }^{\circledR} \mathrm{CTec} 2$ and HTec2) were kindly provided by Novozyme (Franklinton, NC, USA). All the chemicals used in this study were purchased from Fisher Scientfic (Hampton, NH, USA).

\subsubsection{Syntheis of ternary DESs}

$\mathrm{ChCl}$ was dried at $80^{\circ} \mathrm{C}$ for $6 \mathrm{~h}$ before use, other chemicals were used as received. To synthesize ternary DESs, guanidine hydrochloride $(\mathrm{GH}) / \mathrm{ChCl}$ was mixed with EG/ propylene glycol (PG)/glycerol(Gly) and PTSA at a molar ratio of 1:1.94:0.06 in a glass bottle. Then, the mixture was heated at $80{ }^{\circ} \mathrm{C}$ for $2 \mathrm{~h}$ with continuous stirring at $200 \mathrm{rpm}$ until a transparent liquor was formed. A total of six DESs were synthesized and termed as GH:EG/PG/Gly:PTSA and ChCl:EG/PG/Gly:PTSA based on three components in each ternary DES. 


\subsubsection{Pretreatment}

For screening effective DES, $1.5 \mathrm{~g}$ switchgrass was mixed with $13.5 \mathrm{~g}$ DES in a pressure tube (Ace Glass, Vineland, NJ, USA), heated at $120{ }^{\circ} \mathrm{C}$ for $6 \mathrm{~min}$ at 200 rpm in an oil bath, and then cooled down to room temperature. To the pressure tube was added $15 \mathrm{ml}$ acetone/water $(1: 1, \mathrm{v} / \mathrm{v})$, and the slurry was filtered. The solid residue was further washed with $15 \mathrm{ml}$ acetone/water twice, and stored at $-20{ }^{\circ} \mathrm{C}$ before further use. The filtrate and washing liquor were combined, and acetone in the mixed liquid was removed under reduced pressure. The lignin was precipitated after acetone removal, recovered via centrifugation at $10,000 \times \mathrm{g}$ for $5 \mathrm{~min}$, washed with deionized (DI) water four times, and then dried at $45{ }^{\circ} \mathrm{C}$ for $12 \mathrm{~h}$. High solid loading pretreatment at $30 \%$ and $35 \%$ loading was conducted by following the same procedure except that $10 \mathrm{~g}$ switchgrass was loaded into $23.33 \mathrm{~g}$ and $18.57 \mathrm{~g}$ ternary DESs. The mixture was heated at $120{ }^{\circ} \mathrm{C}$ for $20 \mathrm{~min}$ for $30 \%$ solid loading and $30 \mathrm{~min}$ for $35 \%$ solid loading. The mixture was stirred manually every 4 min by using a polytetrafluoroethylene (PTFE) rod. All the pretreatment was conducted at ambient pressure with the glass reactor not capped.

For pretreatment of xylan, $1.5 \mathrm{~g}$ xylan (Beech wood) was put in $13.5 \mathrm{~g}$ ChCl:EG:PTSA and reacted at $120^{\circ} \mathrm{C}$ for $6 \mathrm{~min}$. After cooling down, the mixture was centrifuged at $17,000 \times \mathrm{g}$ for $5 \mathrm{~min}$ to remove any undissolved xylan. To the supernatant was added 3 volume of ethanol, and the thus precipitated xylan was recovered via centrifugation at $17,000 \times \mathrm{g}$ for $5 \mathrm{~min}$. The recovered xylan was washed three times with ethanol and dried at room temperature before further analysis.

\subsubsection{Enzymatic hydrolysis}

Enzymatic hydrolysis was conducted at $2 \%(\mathrm{w} / \mathrm{w})$ in $50 \mathrm{mM}$ citrate buffer (pH 5.5) at $50{ }^{\circ} \mathrm{C}$ for $36 \mathrm{~h}$. The enzyme loading was $5 \mathrm{mg}$ protein/ $\mathrm{g}$ solid with the 
Ctec2 to Htec2 ratio fixed at 10:1. For enzymatic hydrolysis conducted at 20\% (w/w) solid loading, the pretreated switchgrass was loaded at $0,3,9$ and $18 \mathrm{~h}$ with the amount of solid equal to 1/4 of the final solid added each time. Enzymes, citrate buffer, and additional water were loaded at $0 \mathrm{~h}$. The monomeric sugar yield from high solid loading enzymatic hydrolysis was determined according to a previous study (Kristensen et al., 2009).

\subsubsection{Analytical methods}

Compositional analysis of untreated and pretreated switchgrass was conducted following NREL Laboratory Analytical Procedure (Sluiter et al., 2008). Monomeric sugar concentrations were determined using the Agilent 1200 High Performance Liquid Chromatograph (HPLC) system as described in our previous studies (Chen \& Wan, 2018).

For 2D HSQC NMR analysis of lignin, $60 \mathrm{mg}$ lignin sample was dissolved in $0.5 \mathrm{ml}$ DMSO- $d_{6}$, and then analyzed on a Bruker AVIII $800 \mathrm{MHz}$ spectrometer. The set-up of the NMR parameters can be found in our previous study (Chen \& Wan, 2018). Xylan analysis was conducted on the same spectrometer except that $20 \mathrm{mg}$ xylan was dissolved in $0.5 \mathrm{ml} \mathrm{D}_{2} \mathrm{O}$. One drop of $5 \mathrm{M} \mathrm{NaDH}$ was added to $\mathrm{D}_{2} \mathrm{O}$ to facilitate the dissolution of xylan.

Functional groups analysis of xylan was performance on attenuated total reflection Fourier transform infrared spectroscopy (ATR-FTIR) (Nicolet 4700, Thermo Electron, USA). Sample spectra were obtained via 64 scans over the range between 400 and $4000 \mathrm{~cm}^{-1}$. 
4.3. Results and discussion

\subsubsection{Screening effective ternary DESs for pretreatment}

Six DESs were synthesized and their performance in pretreatment were evaluated by comparing xylan and lignin removal, as well as cellulose digestibility. As shown in Figure 4.1, GH:EG:PTSA showed the best performance in terms of lignin and xylan removal. In only 6 min at $120^{\circ} \mathrm{C}, \mathrm{GH}$ :EG:PTSA was able to remove $80 \%$ lignin and xylan from switchgrass. When the EG was replaced by $\mathrm{PG}$, similar delignification rate (80\%) was achieved by GH:PG:PTSA, but the xylan removal was 5\% lower. Compared with GH:EG:PTSA and GH:PG:PTSA, the performance of GH:Gly-PTSA paled as only 10\% lignin was removed. When GH was replaced by $\mathrm{ChCl}$ in these ternary DESs, $\mathrm{ChCl}: \mathrm{EG}: \mathrm{PTSA}$ and $\mathrm{ChCl}$ :PG:PTSA still demonstrated good capability of solubilizing lignin and xylan from switchgrass. In 6 min, ChCl:EG:PTSA removed near 70\% lignin and xylan, while $60 \%$ for $\mathrm{ChCl}: \mathrm{PG}: \mathrm{PTSA}$. The ChCl:Gly:PTSA was not as effective as the other two DESs. For the six DESs, the glucan remained almost intact (less than $2 \%$ loss), suggesting that these ternary DESs selectively removed lignin and hemicellulose. Due to such selectivity, the glucan content reached as high as $75 \%$ for the switchgrass pretreated by GH:EG:PTSA (Figure 4.2). 


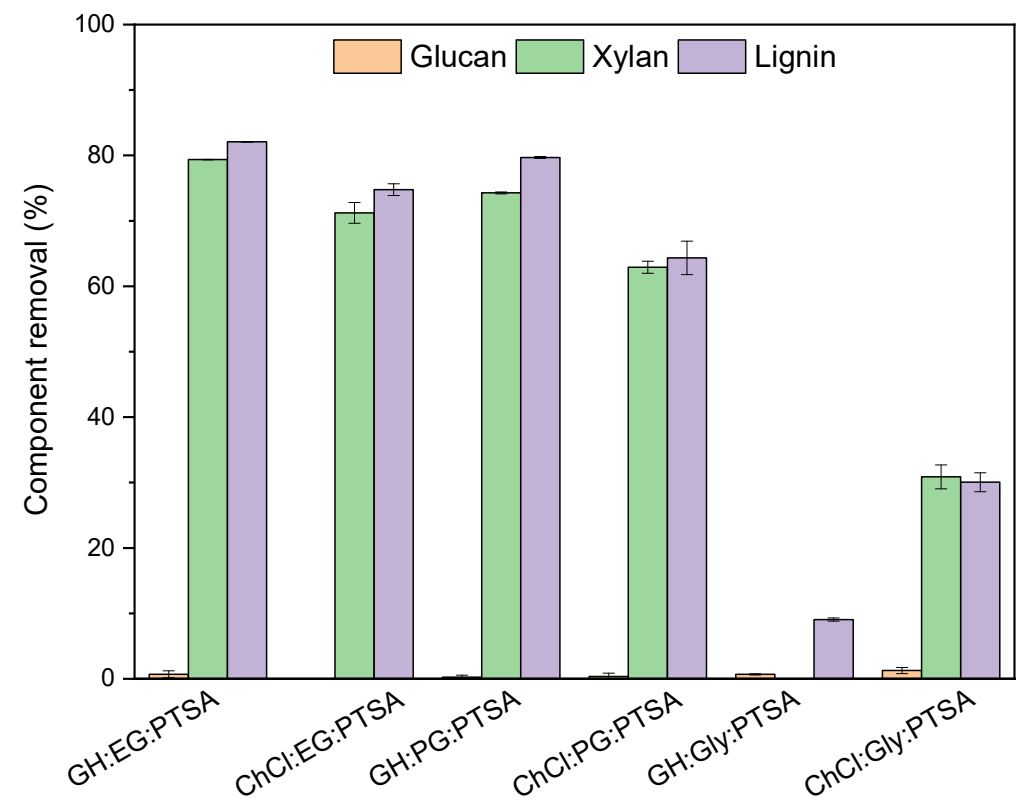

Figure 4.1Component removal of switchgrass upon ternary DESs pretreatment.

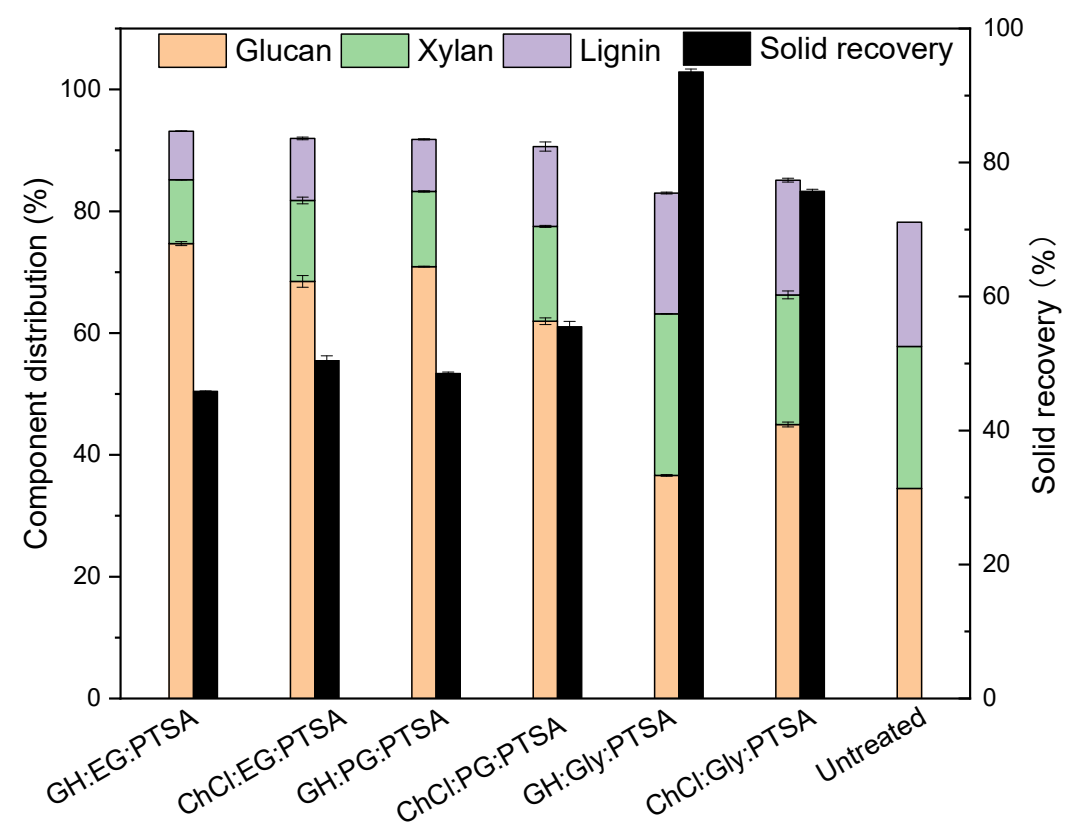

Figure 4.2 Component distribution and solid recovery of switchgrass upon ternary DESs pretreatment.

As another criteria to assess the solvent performance in pretreatment, the digestibility of pretreated switchgrass was examined by conducting enzymatic hydrolysis at $2 \mathrm{wt} \%$ solid loading. As shown in Figure 4.3, 90\% glucose yield was 
obtained after $36 \mathrm{~h}$ for switchgrass pretreated by GH:EG:PTSA, GH:PG:PTSA, and ChCl:EG:PTSA with an enzyme loading of $5 \mathrm{mg}$ protein/g solid, while the glucose yield was decreased to $80 \%$ for ChCl:PG:PTSA. Such difference could be partially contributed to the lower amount of xylan and lignin removed in $\mathrm{ChCl:PG:PTSA}$ pretreated switchgrass. As shown in Figure 4.1, for other three samples with over $90 \%$ glucose yield, the xylan and lignin removal exceeded $70 \%$, while it was about $60 \%$ for ChCl:PG:PTSA. In spite of such difference, compared with the untreated switchgrass, the glucose yield was increased by 5 folds. For other two DESs: GH:Gly:PTSA and ChCl:Gly:PTSA, the glucose yields were only $14 \%$ and 53\%, respectively. The higher lignin and xylan content retained in these two samples could block the access of cellulase to cellulose, and in turn, negatively affected the release of glucose.

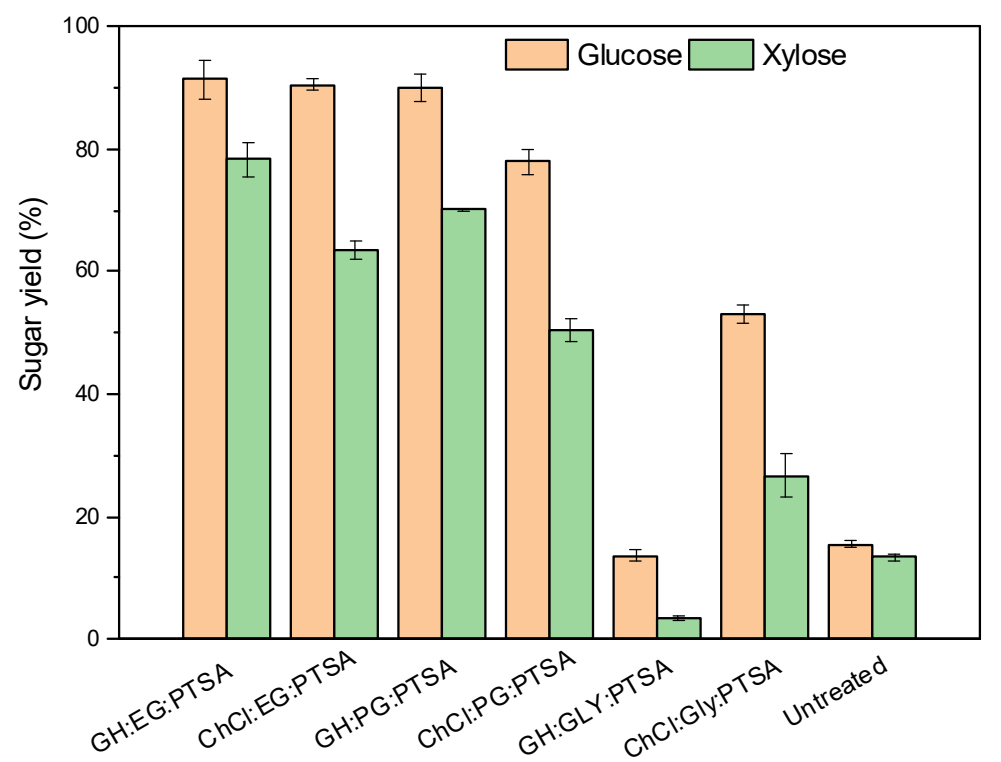

Figure 4.3 Digestibility of switchgrass pretreated by different ternary DESs.

Overall, among the six ternary DESs, four were effective in removing more than $60 \%$ lignin and xylan, and greatly enhanced the cellulose digestibility. Xu et al.(Xu et al., 2016) reported a glucose yield over $90 \%$ of the corn stover pretreated by 
$\mathrm{ChCl}: \mathrm{FA}$ at $130{ }^{\circ} \mathrm{C}$ for $3 \mathrm{~h}$ at $5 \%$ solid loading. Zhang et al. (Zhang et al., 2016a) reported a glucose yield of $80 \%$ from corncob pretreated by $\mathrm{ChCl}$ :LA at $90{ }^{\circ} \mathrm{C}$ for 24 $\mathrm{h}$ at $5 \%$ solid loading. Hou et al.(Hou et al., 2017) reported a glucose yield of $90 \%$ from rice straw pretreated by $\mathrm{ChCl}$ :oxalic acid first and post-treated by $\mathrm{ChCl}$ :Urea for a total of $2 \mathrm{~h}$ at $100{ }^{\circ} \mathrm{C}$ at $5 \%$ solid loading. Compared with these DESs pretreatment, the ternary DESs designed in this study presented a much faster and simpler pretreatment process with doubled solid loading for achieving similar or even better performance.

\subsubsection{Lignin characterization by $N M R$}

To further understand the mechanism of switchgrass fractionation by ternary DESs and the lignin chemical transformation during pretreatment, the recovered lignin samples were characterized by 2D HSQC NMR. The lignin samples from GH:Gly:PTSA and ChCl:Gly:PTSA pretreatment were not characterized as their delignification rates were quite low. The cross signals in the NMR spectra were assigned according to a previous study (Liu et al., 2018), and were presented in Figure 4.4. For the native lignin (NL), $\beta-\mathrm{O}-4$ interunit linkage is the predominant peak in the aliphatic regions, with trace amount of $\beta-5$ and $\beta-\beta$ detected. For lignin recovered from GH:EG:PTSA and GH:PG:PTSA pretreatment, no $\beta-O-4, \beta-5$ and $\beta-\beta$ linkages were detected. When the $\mathrm{GH}$ was replaced by $\mathrm{ChCl}$ in the ternary $\mathrm{DESs}$, the lignin recovered demosntrated some preserved interunit linkages. For ChCl:EG:PTSA, a

trace amount of $\beta-O-4$ linkages was demonstrated, but no peaks for $\beta-5$ and $\beta-\beta$ were shown. The lignin obtained from ChCl:PG:PTSA demonstrated larger peaks for $\beta-\mathrm{O}-4$ and $\beta-5$ linkages, but no peaks for $\beta-\beta$ linkages (Figure $4.4 \&$ Table 4.1). 


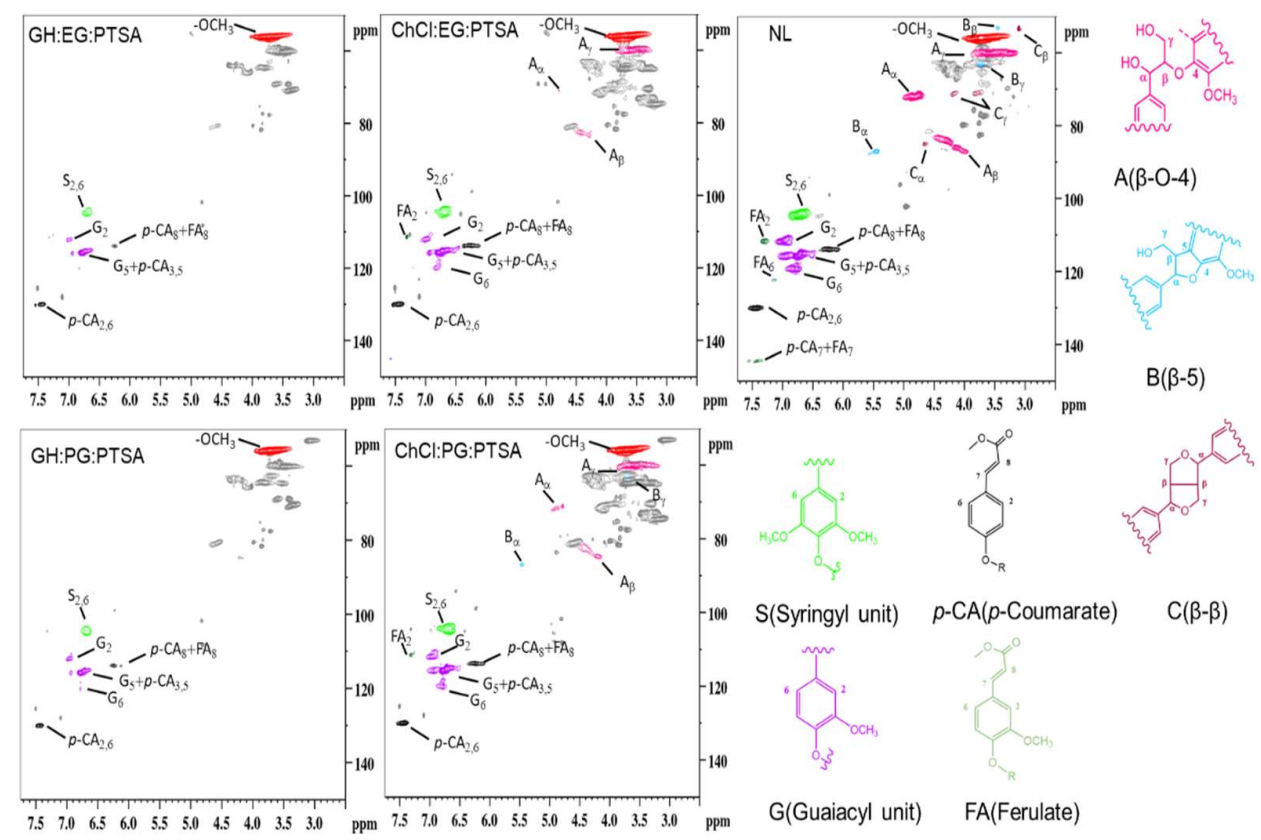

Figure 4.4 2D HSQC NMR spectra of lignin.

Table 4.1 Volume integration of the $\beta-\mathrm{O}-4, \mathrm{FA}, p-\mathrm{CA}, \mathrm{S}$ and $\mathrm{G}$ units in lignin

\begin{tabular}{llcccc}
\hline & NL & GH:EG:PTSA & GH:PG:PTSA & ChCl:EG:PTSA & ChCl:PG:PTSA \\
\hline$\beta-O-4$ & 46.7 & $\backslash$ & $\backslash$ & 2.3 & 23.6 \\
FA & 9.35 & $\backslash$ & $\backslash$ & 5.1 & 8.1 \\
$p$-CA & 33.41 & 28.4 & 25.3 & 25.6 & 20.9 \\
S & 41.2 & 62.5 & 58.9 & 52.8 & 52.3 \\
G & 58.8 & 37.5 & 41.1 & 47.2 & 47.7 \\
S/G & 0.7 & 1.6 & 1.4 & 1.1 & 1.1 \\
\hline
\end{tabular}


In the aromatic region for NL, the dominant peaks were assigned to syringyl unit (S), guaiacyl unit (G), p-coumarate ( $p$-CA) and ferulate (FA). The peaks for these units in all the lignin samples recovered after pretreatment showed different degree of decease. Compared with the $\mathrm{S} / \mathrm{G}$ ratio in $\mathrm{NL}$, the $\mathrm{S} / \mathrm{G}$ ratio in $\mathrm{GH}: \mathrm{EG}: \mathrm{PTSA}$ and GH:PG:PTSA was increased from 0.7 to 1.6 and 1.4 (Table 4.1), and the peaks for $\mathrm{G}_{6}$ disappeared in these two samples. These results were in accordance with those from other studies using ionic liquid and highly concentrated acid pretreatment (Chen et al., 2017; Wen et al., 2014). This is probably because $\mathrm{G}$ unit is more prone to degradation than $\mathrm{S}$ unit in these DESs. The disappearance of cross signal for $\mathrm{G}_{6}$ may also suggest the condensation reaction in this position occurred during pretreatment. For lignin from ChCl:EG:PTSA and ChCl:PG:PTSA, the aromatic units were better preserved, and thus a lower $\mathrm{S} / \mathrm{G}$ ratio than those in lignin from GH:EG:PTSA and GH:PG:PTSA. Especially, lignin from $\mathrm{ChCl:PG:PTSA} \mathrm{presented} \mathrm{more} \mathrm{similarity} \mathrm{to} \mathrm{NL} \mathrm{than} \mathrm{other}$ three lignin. The FA units were absent in GH:EG:PTSA and GH:PG:PTSA, but were still detected in $\mathrm{ChCl}$ :EG:PTSA and $\mathrm{ChCl}: \mathrm{PG}: \mathrm{PTSA}$. In grass, the $\mathrm{FA}$ acts as a bridge connecting lignin with hemicellulose via formation of ether/ester bonds. As discussed earlier, the GH:EG:PTSA and GH:PG:PTSA removed more lignin and hemicellulose than ChCl:EG:PTSA and ChCl:PG:PTSA (Figure 4.1). It suggested that the DESs liberated the hemicellulose and lignin from switchgrass by cleaving the FA-crossed links, and the solubilized lignin experienced further depolymerization and repolymerization upon cleavage of $\beta-\mathrm{O}-4, \beta-5$ and $\beta-\beta$ interunit linkages. With a higher delignification rate, the lignin experienced more structure change.

Overall, the NMR results suggested that varying the components in the ternary DESs could yield lignin with different properties (e.g., more preserved ether bonds, 
higher $\mathrm{S} / \mathrm{G}$ ratio). It is possible to further tune ternary DESs to produce tailor-made lignin with desirable properties to fit different lignin valorization routes.

\subsubsection{Xylan characterization}

Xylan is another major component solubilized in DESs during pretreatment, and understanding its change during pretreatment would be necessary to better understand the mechanisms for lignocellulosic biomass fractionation in ternary DESs. However, unlike lignin, recovery of xylan after pretreatment was difficult.

Alternatively, we pretreated "pure" xylan with ChCl:EG:PTSA and characterized the pretreated xylan via FTIR and NMR. Ternary DESs with GH as a component was not used as adding the antisolvent (i.e., ethanol) to precipitate xylan can cause the precipitation of GH as well, and made recovery of xylan impossible.

The functional groups in treated xylan and pristine xylan were first characterized by FTIR (Figure 4.5). The peak at around $3420 \mathrm{~cm}^{-1}$ was attributed to O$\mathrm{H}$ stretching in the xylan (Harish et al., 2015). The C-H stretching vibration and C-H bending was observed at $2880 \mathrm{~cm}^{-1}$ (Gao et al., 2016). A small peak at $896 \mathrm{~cm}^{-1}$ was assigned to $\beta$-glucosidic bonds between the xylose units, and the peak at $1042 \mathrm{~cm}^{-1}$ suggested the presence of the pyranose form of sugars (Harish et al., 2015). The peak at $1623 \mathrm{~cm}^{-1}$ contributed to the of $\mathrm{C}-\mathrm{C}$ stretching vibration. For both treated and untreated xylan, they presented a similar FTIR spectra, indicating that the recovered xylan did not experience structure change after pretreatment. This was further supported by results from the ${ }^{13} \mathrm{C}$ and 2D HSQC NMR analysis (Fig.4.6\&4.7), which demonstrated the pristine and treated xylan had a similar structure. However, it should be noted that the recovered xylan accounted for less than $10 \%$ of the xylan initially added (data not shown), suggesting that a large amount of xylan may be depolymerized into smaller and unrecoverable oligomers/monomers. Indeed, the HPLC 
analysis of the ChCl:EG:PTSA with xylan dissolved revealed the existence of xylose (Figure 4.8).

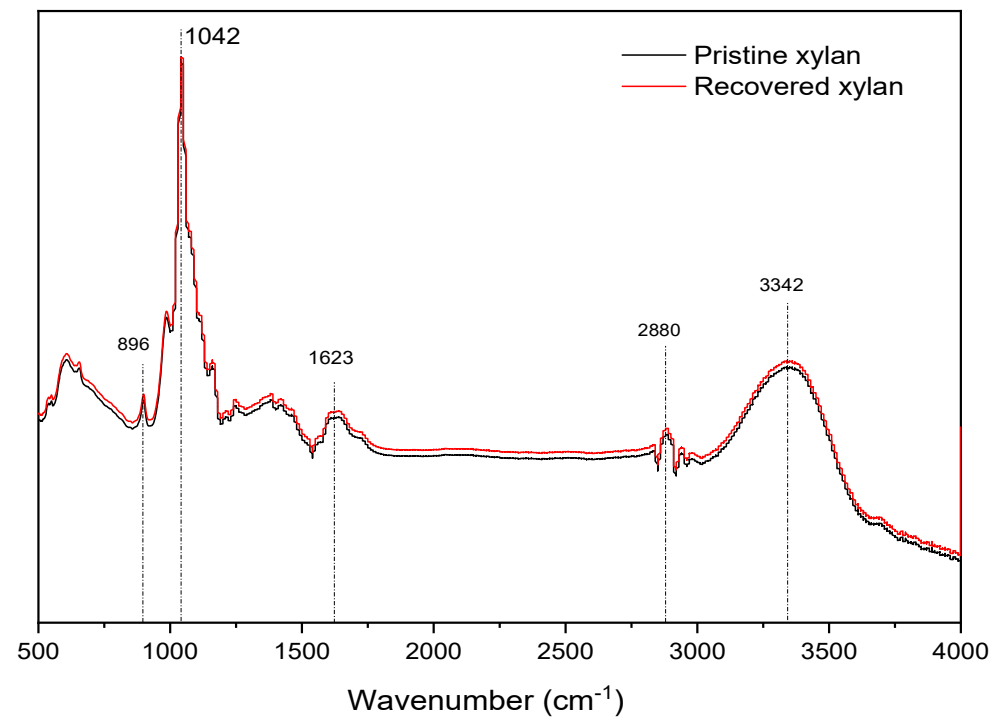

Figure 4.5 FTIR spectra of xylan.
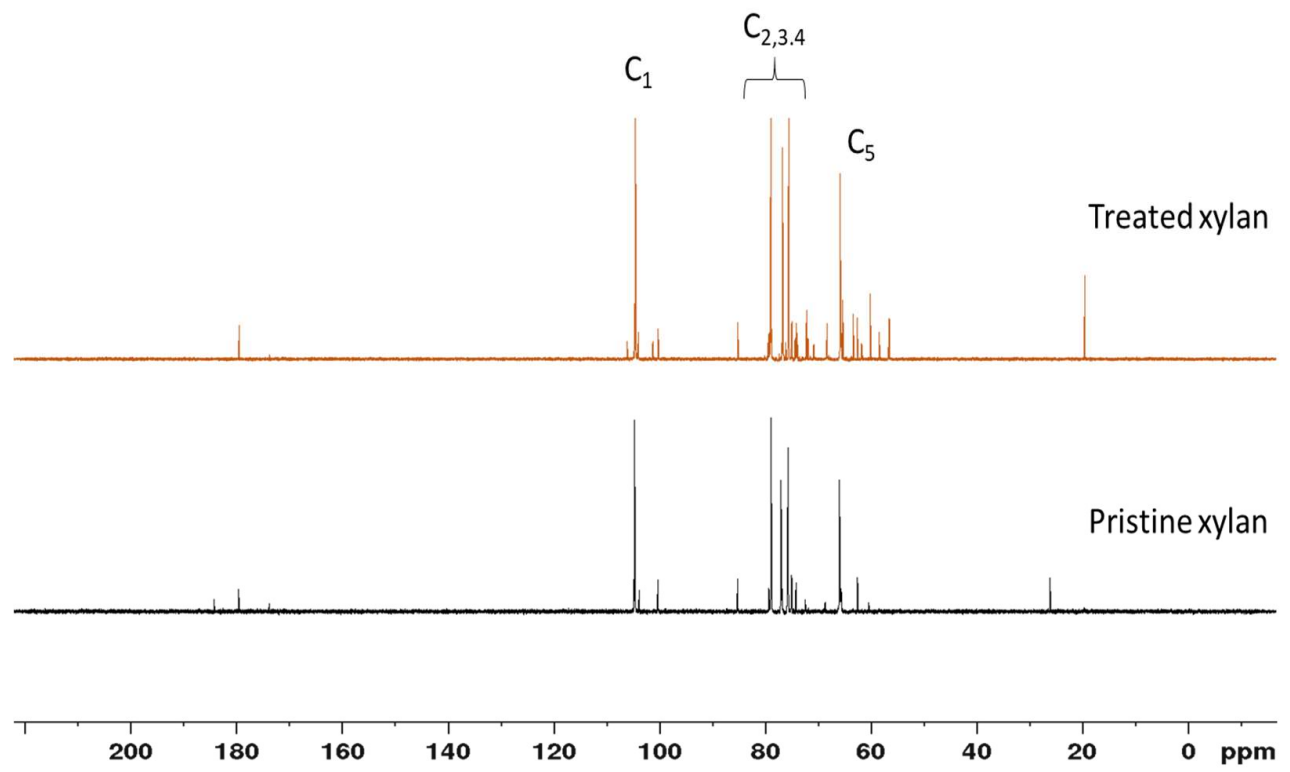

Figure $4.6{ }^{13} \mathrm{C}$ NMR spectra of pristine xylan and treated xylan 

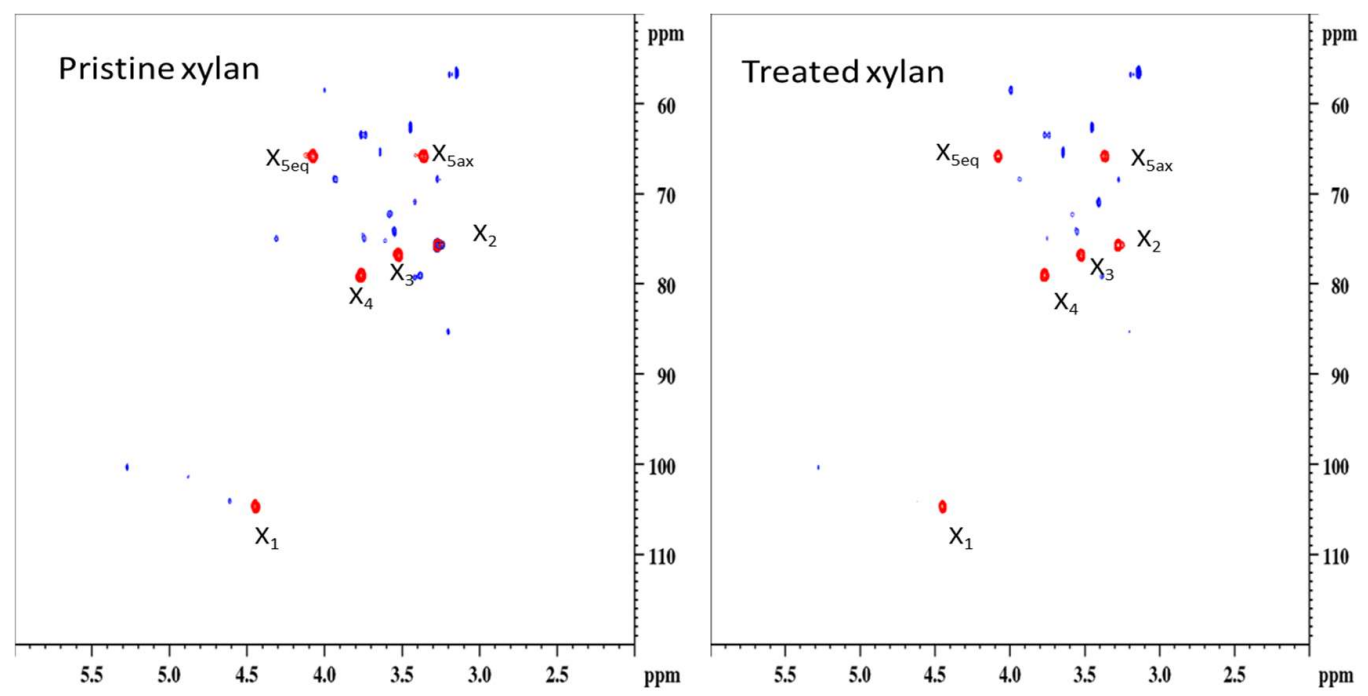

Figure 4.7 2D HSQC NMR spectra of pristine xylan and treated xylan

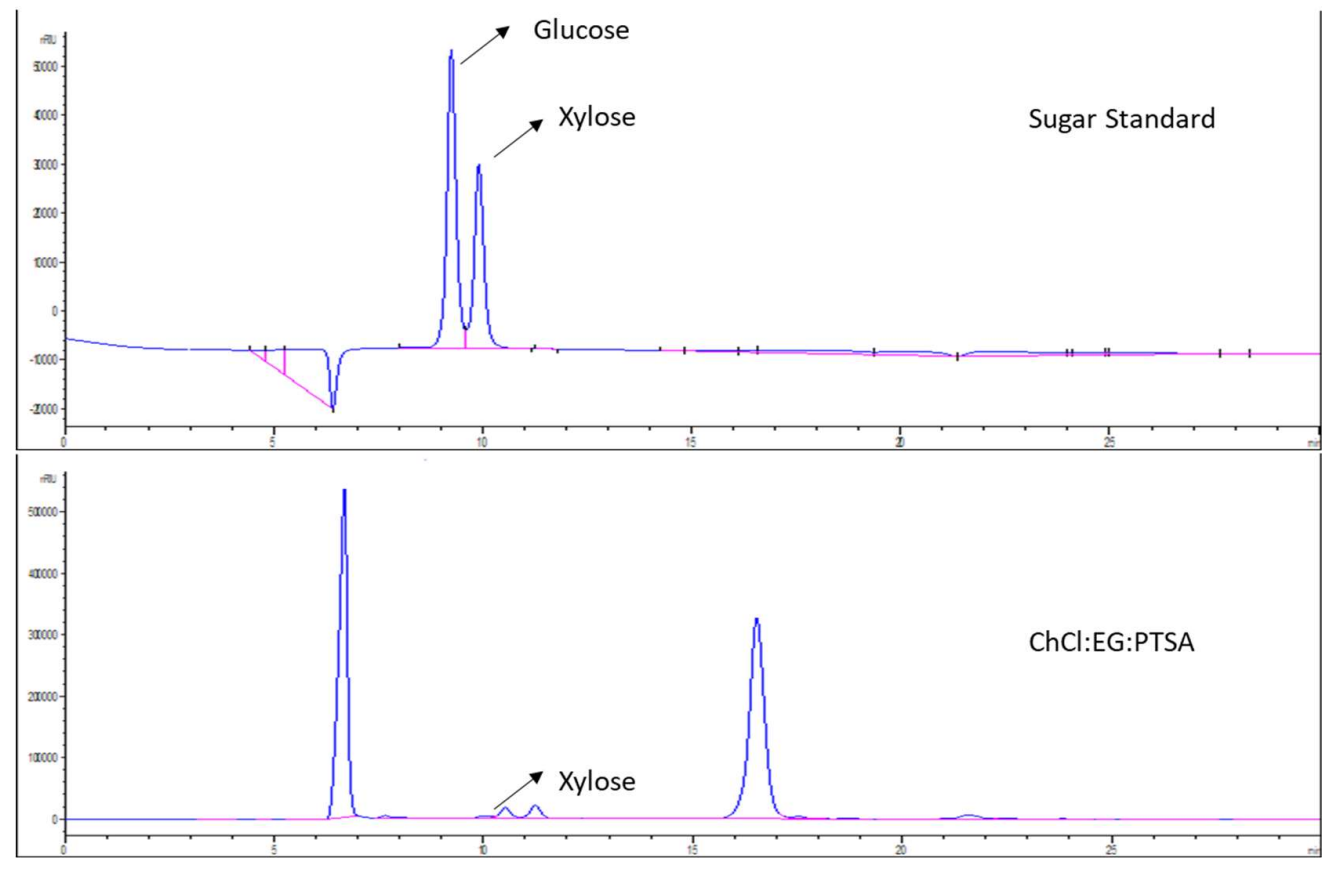

Figure 4.8 HPLC chromatograph of CC-EG-PTSA with xylan dissolved.

Based on above results, the mechanisms for switchgrass fractionation in ternary DESs were proposed in Figure 4.9. In a proton active environment provided by ternary DESs, the ester bonds between lignin and the xylan is first cleaved, leading to the release of xylan and lignin. The dominant $\beta-O-4$ linkages in the solubilized lignin were then protonated at $\alpha$-position, followed by dehydration and formation of active 
benzylic carbon cation. The carbon cation can directly react with the electron rich aromatic ring (e.g., S or G units) in lignin, or undergoes ether bond cleavage to form C3-ketone or C2-aldehyde via different routes. The formed C3-ketone and C2-aldehyde can also react with each other to produce a condensed lignin. The solubilized xylan may undergo depolymerization as well, leading to the formation of xylose. Different from xylan and lignin, the cellulose experience less change in the ternary DESs.

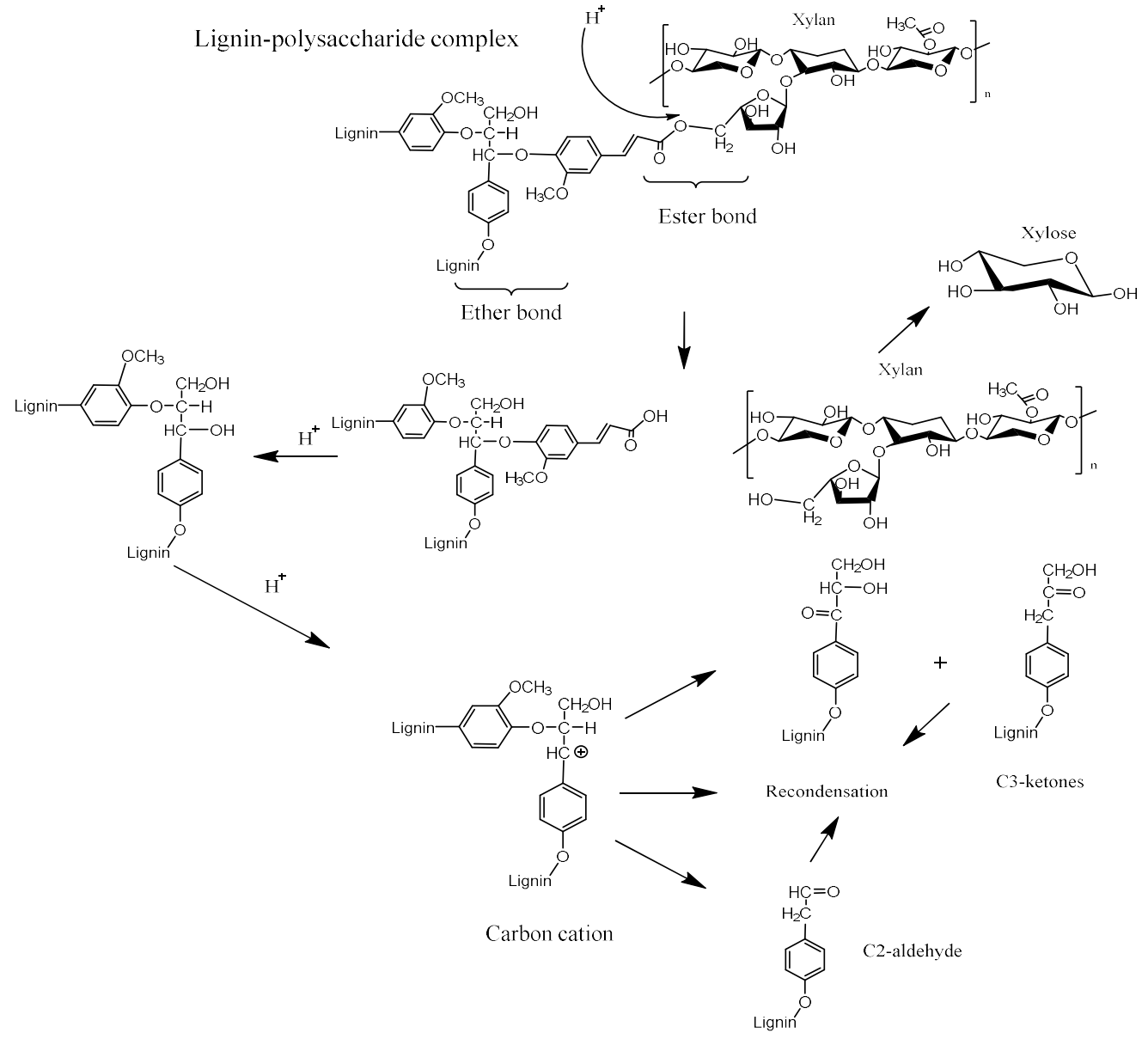

Figure 4.9 Proposed mechanisms for switchgrass fractionation in ternary DESs.

3.4 High solid loading pretreatment and enzymatic hydrolysis

Given the effectiveness of ternary DESs on delignification and enhancing the cellulose digestibility, we further tested their effectiveness at 30\% and $35 \%$ solid loading. Two DESs: GH:EG:PTSA and ChCl:EG:PTSA, were selected for evaluation. 
Under 30\% solid loading, GH:EG:PTSA was still very effective in removing about $75 \%$ lignin and xylan from switchgrass at $120{ }^{\circ} \mathrm{C}$ for only 20 min (Figure 4.10 ).

Similar to what observed at low solid loading pretreatment, $\mathrm{ChCl}$ :EG:PTSA was less effective than GH:EG:PTSA, but was still able to remove as high as $65 \%$ lignin and xylan. Increasing the solid loading to $35 \%$ resulted in a slight decrease of lignin and xylan removal, but the removal rate still maintained above 60\% (Figure 4.10).

Overall, both GH:EG:PTSA and ChCl:EG:PTSA were highly effective for lignin and xylan removal at a solid loading up to $35 \%$. The effective removal of xylan and lignin resulted in a cellulose rich solid residue with a content over $60 \%$ (Figure 4.11 ). To the best of our knowledge, this is the first study demonstrating a $60 \%$ high delignification rate under $35 \%$ solid loading for $30 \mathrm{~min}$ at $120^{\circ} \mathrm{C}$ using DES based pretreatment. Although similar delignification were achieved by $\mathrm{ChCl}: \mathrm{FA}$ in a previous study, the solid loading used was only $5 \%$ while the pretreatment time were $3 \mathrm{~h}$ at $120{ }^{\circ} \mathrm{C}(\mathrm{Xu}$ et al., 2016).

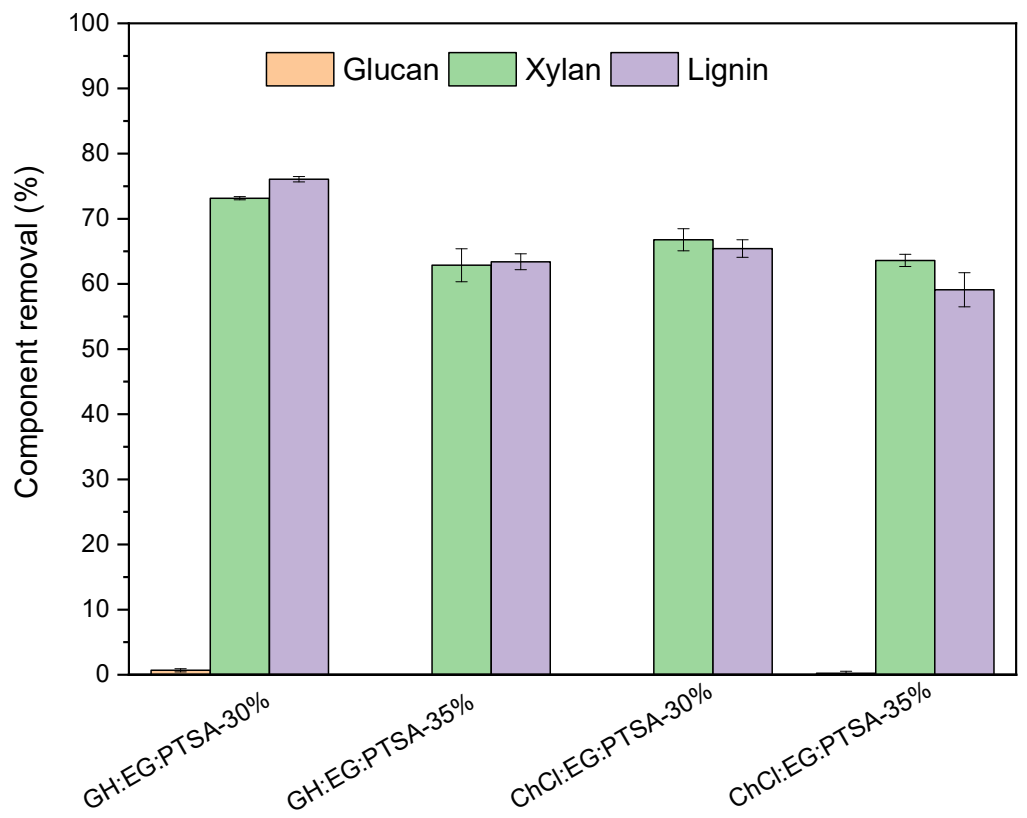

Figure 4.10 Component removal of switchgrass after high solid loading pretreatment. 


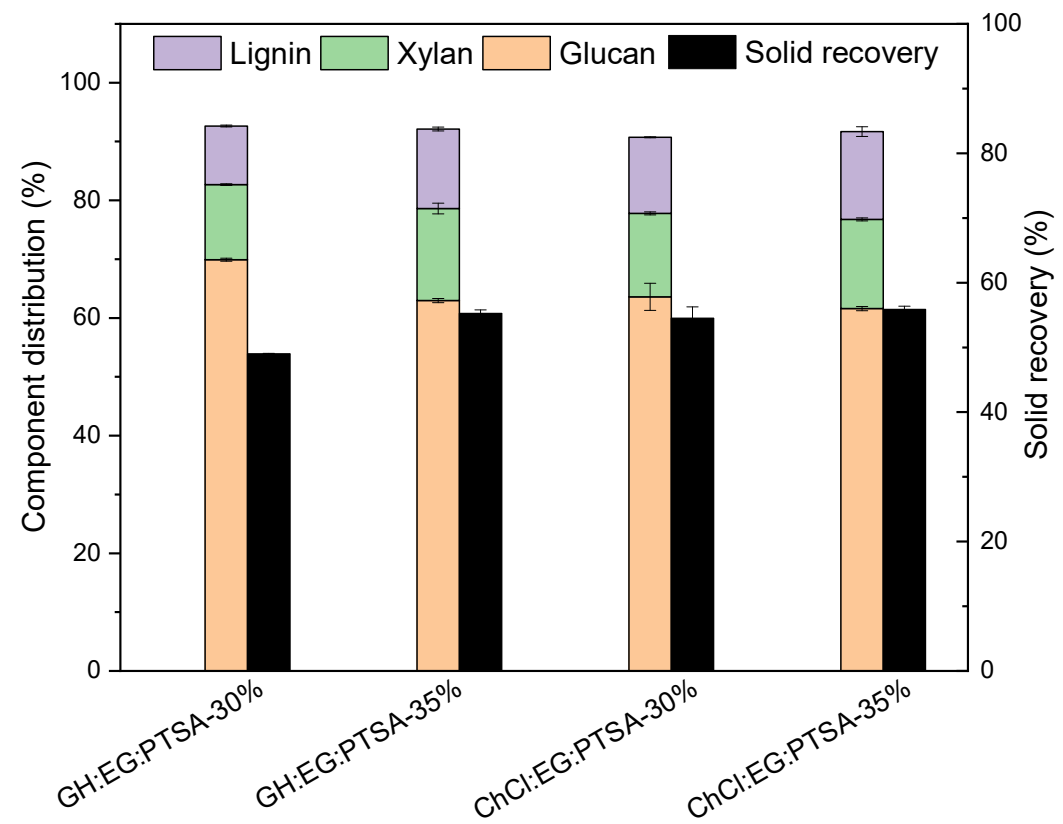

Figure 4.11 Component distribution and solid recovery of switchgrass after high solid loading pretreatment.

As discussed above, to reduce the cost with DESs based pretreatment, high solid loading enzymatic hydrolysis is desired. To this end, we further evaluated the digestibility of the pretreated switchgrass at $20 \%(\mathrm{w} / \mathrm{w})$ solid loading. As shown in Figure 4.12, among the four pretreated switchgrass, the one pretreated by ChCl:EG:PTSA at $30 \%(\mathrm{w} / \mathrm{w})$ solid loading gave the highest glucose yield of $78.4 \%$, and a glucose concentration of $128 \mathrm{~g} / \mathrm{L}$. The one pretreated by GH:EG:PTSA at $30 \%(\mathrm{w} / \mathrm{w})$ solid loading presented a reduced glucose yield $(72 \%)$, but a glucose concentration of $130 \mathrm{~g} / \mathrm{L}$ was still obtained due to a higher cellulose content in the sample (Figure 4.12). For switchgrass pretreated at $35 \%(\mathrm{w} / \mathrm{w})$ solid loading, the digestibility was lower than those from their counterparts at 30\% solid loading, but $72 \%$ glucose yield was still obtained for the one from $\mathrm{ChCl}$ :EG:PTSA pretreatment. Zhou et al. (Zhou et al., 2016a) reported a glucose yield of $80 \%$ from $\gamma$-valerolactone pretreated reed stover at 20\% solid loading enzymatic hydrolysis using an enzyme loading of $20 \mathrm{~g}$ protein/g cellulose after $120 \mathrm{~h}$. In another study, Chen et al. (Chen et al., 2016) 
reported a glucose yield of $77 \%$ from DMR (deacetylation and mechanical refining) treated corn stover using an enzyme loading of $20 \mathrm{mg}$ protein/ $\mathrm{g}$ cellulose at $25 \%$ solid loading. Using a similar solid loading and enzymatic hydrolysis time, the switchgrass pretreated by $\mathrm{ChCl}$ :EG:PTSA at 30\% solid loading gave a similar glucose yield, but with a lower enzyme loading (equal to $8 \mathrm{mg}$ protein/g cellulose). Correspondingly, a hydrolysate containing a total of $148 \mathrm{~g} / \mathrm{L}$ monomeric sugar (128 g/L glucose and $20 \mathrm{~g} / \mathrm{L}$ xylose) was produced. Assuming ethanol yield of $0.45 \mathrm{~g} / \mathrm{g}$ glucose and $0.30 \mathrm{~g} / \mathrm{g}$ xylose (Liu et al., 2014), this concentrated hydrolysate could yield $64 \mathrm{~g} / \mathrm{L}$ ethanol. Producing cellulosic ethanol with a titer exceeding industrially relevant level $(>50 \mathrm{~g} / \mathrm{L})$ could reduce the downstream processing cost and other operating costs, but remains to be challenging due to difficulties in obtaining highly concentrated sugar hydrolysate from high solid enzymatic hydrolysis (Chen et al., 2016; Nguyen et al., 2016). The proposed high solid loading pretreatment and enzymatic hydrolysis in this study has great potential to overcome such challenges. It is also expected that by further optimizing the enzymatic hydrolysis, a hydrolysate with sugar concentrations higher than those presented in this study could be obtained. 


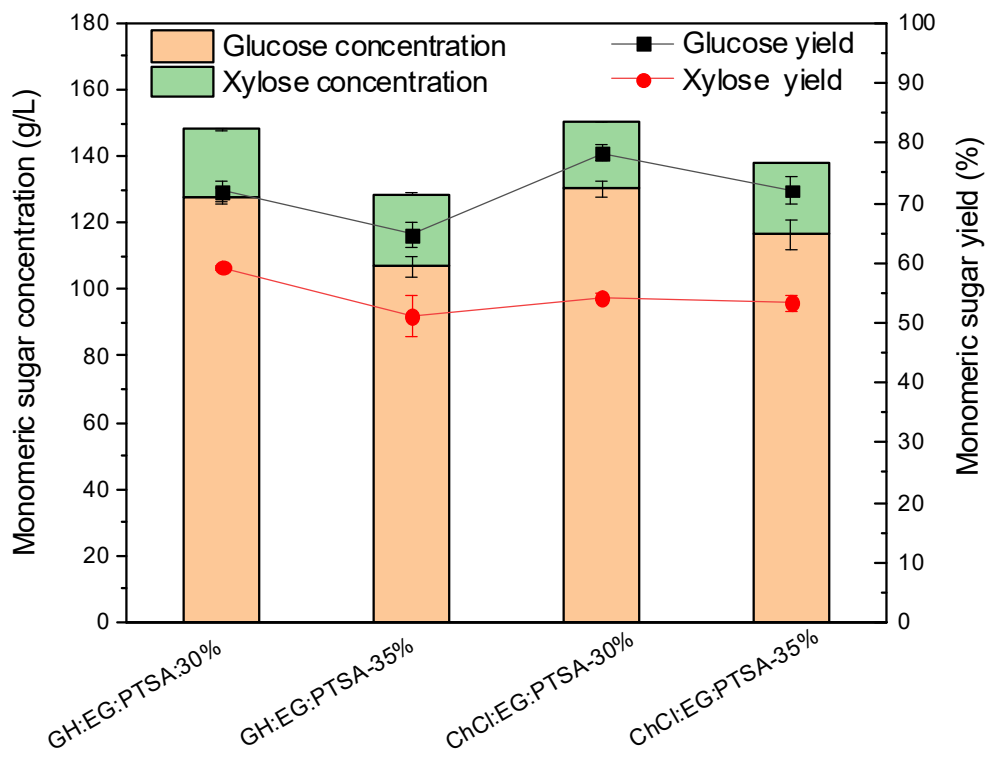

Figure 4.12 Digestibility of switchgrass and sugar concentration from $20 \%(\mathrm{w} / \mathrm{w})$ solid loading enzymatic hydrolysis.

\subsection{Conclusion}

A series of ternary DESs were synthesized and screened for pretreating switchgrass at $10 \mathrm{wt} \%$ solid loading. GH:EG/PG:PTSA and ChCl:EG/PG:PTSA were highly effective in removing lignin and xylan, with at least 64\% lignin and 63\% xylan removed in only 6 min at $120^{\circ} \mathrm{C}$. Characterization of recovered lignin by $2 \mathrm{D}$ HSQC NMR revealed that the effective cleavage of the ether and ester bonds linked xylan and lignin resulted in the fast fractionation of switchgrass. GH:EG:PTSA and ChCl:EG:PTSA still showed excellent performance even under $30 \%$ and $35 \%$ solid loading pretreatment, with more than $60 \%$ lignin and xylan removed. High solid loading (20\%) enzymatic hydrolysis of these pretreated switchgrass produced a highly concentrated sugar hydrolysate. Under the best scenario, a glucose yield of $78.4 \%$ and a sugar hydrolysate containing a total of $148 \mathrm{~g} / \mathrm{L}$ monomeric sugar were obtained after $120 \mathrm{~h}$ with an enzyme loading of $5 \mathrm{mg}$ protein/g solid. In sum, this study provided a novel, efficient, and promising process for pretreating lignocellulosic biomass at high solid loading. 


\section{Disclosure}

The following chapter was based on the paper that has been published on the journal of ACS Sustainable Chemistry and Engineering. Reproduced with permission from (Chen et al., 2018b). Copyright (2018) American Chemical Society. Wesley Reznicek was the co-author, and provided help discussion. 


\section{Chapter 5 Aqueous Choline Chloride: a Novel Solvent for Switchgrass Fractionation and Subsequent Hemicellulose Conversion into Furfural}

\subsection{Introduction}

Lignocellulosic biomass contains three major components, namely, cellulose, hemicellulose, and lignin. Pretreatment often aims at maximizing cellulose digestibility for sugar production while leaving lignin untapped. A typical fate of lignin in a biorefinery plant is being burned as a low quality solid fuel for the plant. Recently lignin valorization has been recognized pivotal for an economically viable biorefinery. The concept of "lignin-first" pretreatment is thus proposed, targeting both maximum sugar and lignin yields while ensuring lignin valorization potential (Renders et al., 2017). For pretreatment liquor, it is often rich in hemicellulosederived products, but generally discharged as a liquid waste stream. Upgrading pretreatment liquor would not only improve the economic viability of lignocellulosebased biorefinery, but also minimize the discharge of liquid pretreatment waste.

Therefore, new pretreatment strategies need to be developed for achieving the goal of "lignin-first" while minimizing waste generation.

Lignin is the most abundant aromatic resource on earth and comprises mainly methoxylated phenylpropanoid subunits (Ragauskas et al., 2014b; Rinaldi et al., 2016). It is a promising substrate for aromatic chemicals and drop-in transportation fuels. However, upgrading lignin into aromatics is generally limited by (1) ineffective extraction of lignin from lignocellulose; (2) undesired condensation of lignin upon the cleavage of ether bonds during extraction (Renders et al., 2017; Sathitsuksanoh et al., 2014). Dilute acid pretreatment is an example that cleaves inter-unit ether bonds, resulting in condensed lignin (Sturgeon et al., 2013). Therefore, different strategies 
have been proposed to prevent lignin condensation. One approach is active stabilization of ether bonds, such as adding formaldehyde to biomass pretreatment, which has been shown to be effective for preserving ether bonds in beech lignin and poplar lignin (Shuai et al., 2016a). The resultant lignin gave near theoretical yields of guaiacyl and syringyl monomers via hydrogenolysis depolymerization. However, side reactions between formaldehyde and cellulose led to low digestibility of pretreated solids. Therefore, it is desirable to develop a pretreatment that will enable the extraction of valorizable lignin while improving cellulose digestibility of pretreated biomass.

Choline chloride $(\mathrm{ChCl})$ is renewable, mass-produced, and commonly used as an animal feed ingredient and a hydrogen bond acceptor for deep eutectic solvents (DESs) (Loow et al., 2017; Lynam et al., 2017; Morandeira et al., 2017). DESs comprising $\mathrm{ChCl}$ and carboxylic acids (e.g., formic acid, lactic acid, acetic acid) have been exploited for lignocellulose fractionation, showing outstanding capability of improving cellulose digestibility (Xu et al., 2016; Zhang et al., 2016a). Lignin structures and properties upon DES pretreatment were also investigated. One example is wood lignin extracted by ChCl:lactic acid DES, which had low molecular weight and uncondensed structure upon complete cleavage of ether bonds (Alvarez-Vasco et al., 2016). Water, when added at a certain amount, plays a positive role in DES pretreatment, such as reducing cellulose loss and improving lignin extraction (Kumar et al., 2016a). While it is well perceived that water can tune DES chemistry, how water in a DES could affect lignin chemistry remains unclear. There is also no study ever done using $\mathrm{ChCl}$ solution rather than neat or aqueous ChCl-based DESs for biomass pretreatment. If $\mathrm{ChCl}$ solution is proved to be an effective pretreatment solvent, it would not only provide insights into individual roles of $\mathrm{ChCl}$ and water in 
aqueous ChCl-based DESs for biomass fractionation, but also open a new avenue to the use of $\mathrm{ChCl}$ for biomass processing.

The objective of this study was to explore a novel acidified $\mathrm{ChCl}$ solution for biomass pretreatment. The recyclability of the solvent was also studied in order to save pretreatment cost and to avoid unnecessary waste generation. To test the "ligninfirst" concept based on $\mathrm{ChCl}$ solution, individual fractions resulting from switchgrass breakdown were investigated, including solid residues (pulp), solubilized lignin, and xylose-rich pretreatment liquor. Specifically, we evaluated the pulp digestibility, characterized lignin chemistry, and tested the feasibility for upgrading pretreatment liquor into furfural.

\subsection{Experimental section}

\subsubsection{Materials}

Switchgrass was collected from South Farm of University of Missouri in Columbia, MO, air-dried, and ground through a 40-mesh screen. It contained $31.57 \%$ glucan, 19.11\% xylan, and 20.35\% lignin. All chemicals were purchased from Fisher Scientific (Hampton, NH, USA), and hydrolytic enzymes (Cellic ${ }^{\circledR}$ CTec2 and HTec2) were kindly provided by Novozyme (Franklinton, NC, USA).

\subsubsection{Pretreatment}

Pretreatment was conducted at $120^{\circ} \mathrm{C}$ for 25 min with magnetic stirring of 200 rpm in a pressure glass tube sealed with a PTFE plug (\#8648-30 and \#5845-47, respectively, Ace Glass, Vineland, NJ, USA). The solid to liquid weight ratio for the pretreatment was $1: 10$, namely $1.5 \mathrm{~g}$ dry switchgrass to $15 \mathrm{~g}$ solvent. The pretreatment solvent was acidified $\mathrm{ChCl}$ solution comprising $75 \mathrm{wt} \% \mathrm{ChCl}$ and $1.0 \mathrm{wt} \% \mathrm{H}_{2} \mathrm{SO}_{4}$. The pretreatments using non-acidified $75 \mathrm{wt} \% \mathrm{ChCl}$ solution and $1.0 \mathrm{wt} \% \mathrm{H}_{2} \mathrm{SO}_{4}$ 
solution, respectively, were also conducted for comparison. The $\mathrm{pH}$ values of the solvents were measured using a Mettler Toledo FEP20 pH meter (Mettler Toledo, Columbus, $\mathrm{OH}, \mathrm{USA})$ at room temperature $\left(22.5^{\circ} \mathrm{C}\right)$. Upon completion of reaction, the pressure tube was taken out of the oil bath and cooled down to room temperature. Then, $15 \mathrm{ml}$ acetone: water $(1: 1, \mathrm{v} / \mathrm{v})$ was added to the pretreatment slurry followed by vacuum filtration. The retained solid was washed with $15 \mathrm{ml}$ acetone: water (1:1, $\mathrm{v} / \mathrm{v}$ ) twice and then stored at $-20{ }^{\circ} \mathrm{C}$ prior to further use. The filtrate collected from the slurry separation and solid washing was combined, and vacuum-evaporated to remove acetone, resulting in the precipitation of lignin. The precipitated lignin was collected via centrifugation at $10,000 \times \mathrm{g}$ for $5 \mathrm{~min}$, washed with deionized (DI) water three times, and then dried at $45^{\circ} \mathrm{C}$. The supernatant was also collected, condensed via vacuum evaporation until it reached the same amount of the original pretreatment solvent, and then reused for a new pretreatment cycle. For the fourth and fifth pretreatment cycles, $\mathrm{H}_{2} \mathrm{SO}_{4}$ was supplemented at $0.7 \mathrm{wt} \%$ and $0.4 \mathrm{wt} \%$ to the recycled solvent, respectively, to adjust the solvent $\mathrm{pH}$ to the level of freshly prepared acidified $\mathrm{ChCl}$ solution $(\mathrm{pH}=1.11)$. The schematic diagram of the pretreatment process is shown in Figure 5.1. All experiments were done in duplicate, and data are reported as mean values with standard deviation.

Lignin yield was calculated as recovered lignin with respect to solubilized lignin in a single cycle (Equation 1), and cumulative lignin yield was calculated based on the cumulative pretreatment cycles (Equation 2):

Lignin yield $(\%)=\frac{\text { Lignin recovered from the } n^{\text {th }} \text { cycle }}{\text { Lignin solublized in the } n^{\text {th }} \text { cycle }} \times 100 \%$

$$
\text { Cumulative lignin yield (\%) }=\frac{\sum_{i=1}^{n} \text { Lignin recovered from Cycle } i}{\sum_{i=1}^{n} \text { Lignin solublized in Cycle } i} \times 100 \%
$$


where $n$ is the number of pretreatment cycles $(1 \leq n \leq 5)$.

\subsubsection{Enzymatic hydrolysis}

Enzymatic hydrolysis of biomass was carried out in $50 \mathrm{~mL}$ Erlenmeyer flask at $50{ }^{\circ} \mathrm{C}, 150 \mathrm{rpm}$ for $48 \mathrm{~h}$. The sample was added at $1.5 \mathrm{wt} \%$ to $20 \mathrm{~mL}$ citrate buffer $(50$ $\mathrm{mM}, \mathrm{pH}$ 5.5) supplemented with $0.01 \mathrm{wt} \% \mathrm{NaN}_{3}$. CTec 2 was loaded at $20 \mathrm{mg}$ protein/g solid and HTec2 at $2 \mathrm{mg} / \mathrm{g}$ solid. After hydrolysis, the hydrolysate was boiled for $5 \mathrm{~min}$ to deactivate the enzymes, and then centrifuged at $17,000 \times \mathrm{g}$ for 2 min. The supernatant was collected, and stored at $-20{ }^{\circ} \mathrm{C}$ prior to HPLC analysis. The results are reported as mean values with standard deviation from two independent experiments. 


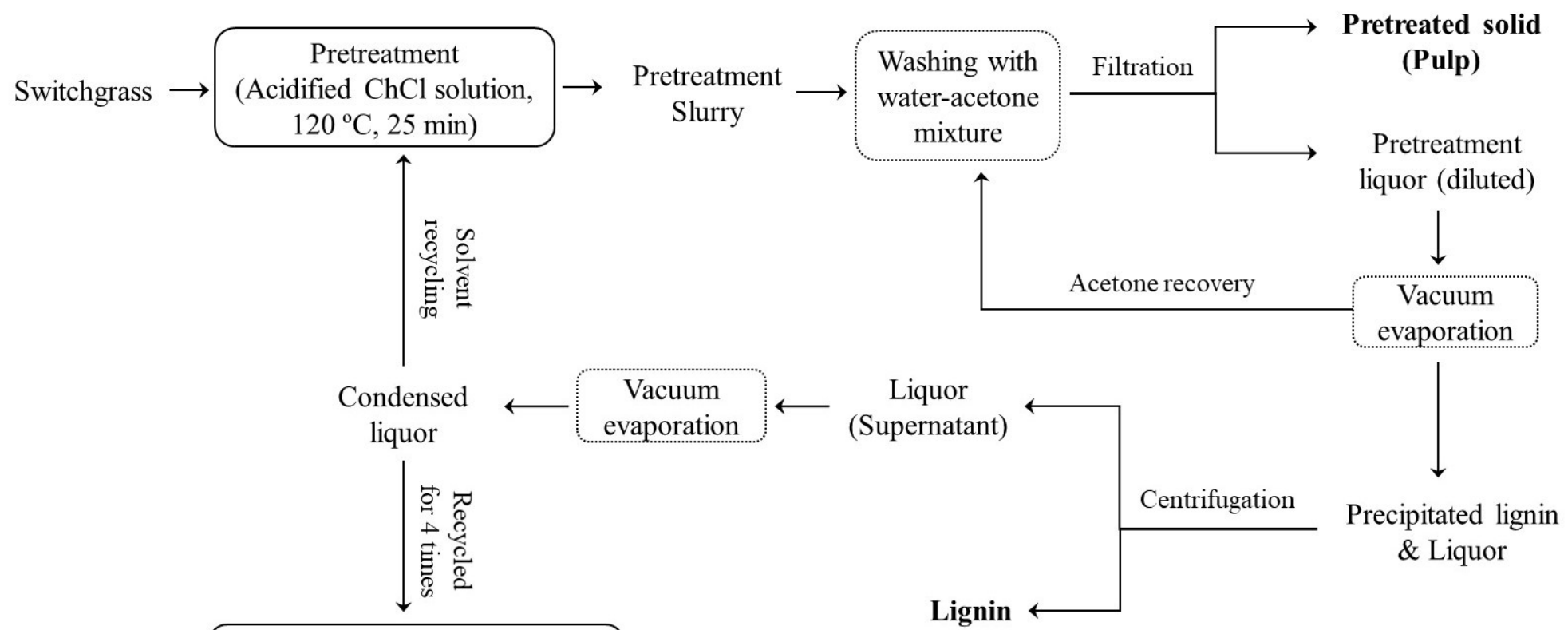

\section{Furfural $\longleftarrow\left(\begin{array}{c}\text { Furfural Production } \\ \left(2 \% \mathrm{w} / \mathrm{v} \mathrm{AlCl}, 160^{\circ} \mathrm{C}, 15 \mathrm{~min}\right)\end{array}\right.$}

Figure 5.1 Schematic diagram of the proposed biomass pretreatment and furfural production process. 


\subsubsection{Furfural production}

The pretreatment liquor collected from the final pretreatment cycle (Cycle 5) was tested for furfural production. The temperature $\left(130-160{ }^{\circ} \mathrm{C}\right), \mathrm{AlCl}_{3}$ addition $(2 \%$ $\mathrm{w} / \mathrm{v})$, and reaction time (15-50 $\mathrm{min})$ were first optimized using freshly prepared acidified $\mathrm{ChCl}$ solution containing $50 \mathrm{~g} / \mathrm{L}$ pure xylose. Furfural production from the pretreatment liquor was then performed under the optimized condition. In a typical run, $5 \mathrm{~mL}$ xylose-containing $\mathrm{ChCl}$ solution or pretreatment liquor was added to a pressure tube sealed with a PTFE stopper as aforementioned. The pressure tube was then placed in a preheated oil bath at a target temperature for a predetermined time. After reaction, the tube was taken out of the oil bath immediately and cooled down to room temperature. The liquid was collected and analyzed for furfural concentration. Furfural yield was calculated as the molar ratio of furfural produced to xylose initially contained in a reaction medium. Furfural titer and yield are reported as mean values with standard deviation from two independent experiments.

\subsubsection{Analytical methods}

The compositional analysis of biomass was conducted following the NREL protocol.(Sluiter et al., 2008) Sugar monomers were analyzed by high performance liquid chromatography (HPLC) Agilent 1200 series (Agilent Technologies, Palo Alto, CA, USA) equipped with a refractive index detector and an Aminex HPX-87P column $(300 \times 7.8 \mathrm{~mm})($ Bio-Rad, Hercules, CA, USA). The reflective index detector (RID) and column temperatures were maintained at 35 and $80{ }^{\circ} \mathrm{C}$ respectively, and the mobile phase was HPLC grade water, eluting at a flow rate of $0.6 \mathrm{~mL} / \mathrm{min}$. Furfural was determined using the same HPLC system equipped with a ZORBAX Eclipse XDB-C18 column $(150 \times 4.6 \mathrm{~mm})($ Agilent Technologies, Palo Alto, CA, USA). The 
column temperature was maintained at $30{ }^{\circ} \mathrm{C}$. The mobile phase was $10 \mathrm{vol} \%$ acetonitrile, eluting at a flow rate of $1 \mathrm{~mL} / \mathrm{min}$.

Powder X-ray diffraction (XRD) data were acquired on a Scintag X2 diffractometer (Scintag, Inc., Cupertino, CA, USA) equipped with a $\mathrm{Cu} / \mathrm{K} \alpha$ sealed tube X-ray generator (operated at $45 \mathrm{kV}, 40 \mathrm{~mA}$ ) and a Peltier-cooled solid state scintillation detector. The samples were loaded into a $1 \mathrm{~mm}$ deep PMMA dish and leveled using a glass microscope slide. Scans were collected at $2 \theta$ of $5-50^{\circ}$ at a scanning rate of $2 \%$ min and a resolution of $0.02^{\circ}$ per data point. Cellulose crystallinity (crystallinity index, CrI) was calculated based on the following equation:(Segal et al., 1959)

$$
\operatorname{CrI}(\%)=\frac{\left[I_{002}-I_{a m}\right]}{I_{002}} \times 100
$$

where $I_{002}$ is the maximum intensity of diffraction peak at $2 \theta$ around $22^{\circ}$, and $I_{a m}$ is the minimum intensity of amorphous portion at $2 \theta$ of $18.0-19.0^{\circ}$.

Attenuated total reflection Fourier transform infrared spectroscopy (ATR-FTIR) (Nicolet 4700 FT-IR, Thermo Electron Co., Waltham, MA, USA) was used to analyze the functional groups of untreated and pretreated biomass. Sample spectra were obtained via 64 scans over the range between 400 and $4000 \mathrm{~cm}^{-1}$.

The detailed chemistry of both native lignin and lignin recovered from different pretreatment cycles was analyzed by 2D HSQC NMR. Native lignin was prepared following a previously reported method with some modification.(Sathitsuksanoh et al., 2014) In brief, extractive-free switchgrass was ball-milled using a ceramic jar, and then subjected to enzymatic hydrolysis for $48 \mathrm{~h}$. The resultant solid residues were washed with water and $6 \mathrm{M}$ guanidine hydrochloride sequentially, and then air-dried. 
The dried solids were further extracted using dioxane:water $(96: 4, \mathrm{v}: \mathrm{v})$ containing 0.04 $\mathrm{N} \mathrm{HCl}$ for $24 \mathrm{~h}$, and then collected as native lignin. Two dimensional ${ }^{1} \mathrm{H}-{ }^{13} \mathrm{C}$ chemical shift correlation HSQC NMR was acquired on a Bruker AVIII $800 \mathrm{MHz}$ spectrometer using Bruker supplied pulse sequence named hsqcetgp (Bruker, Billerica, MA, USA). About $60 \mathrm{mg}$ sample was dissolved in $0.5 \mathrm{~mL}$ DMSO- $\mathrm{d}_{6}$ and the solution was transferred to a $5 \mathrm{~mm}$ NMR tube. The temperature of the sample was kept at $50{ }^{\circ} \mathrm{C}$ throughout the NMR acquisition. The spectral width is $15.4 \mathrm{ppm}$ for ${ }^{1} \mathrm{H}$ and 170.0 ppm for ${ }^{13} \mathrm{C}$. A total of $2048\left({ }^{1} \mathrm{H}\right)$ complex points were acquired with $256\left({ }^{13} \mathrm{C}\right)$ time increments. The ${ }^{1} \mathrm{~J}_{\mathrm{CH}}$ was set to be $145 \mathrm{~Hz}$ which is the average one-bond $\mathrm{C}-\mathrm{H}$ coupling constant. The number of scans was 48 and the repetition delay was $1.5 \mathrm{~s}$. The ${ }^{13} \mathrm{C}$ dimension was zero-filled to 2048 points before the data were subjected to a sine-squared window function (shifted $90^{\circ}$ ) and Fourier transformation. The chemical shift axes were calibrated with respect to the solvent signals $(2.49 \mathrm{ppm}$ for residual proton and $39.5 \mathrm{ppm}$ for ${ }^{13} \mathrm{C}$ ). Semi-quantification of $\beta-\mathrm{O}-4$ was performed using Equations (4) and (5):

$$
\begin{aligned}
& \mathrm{I}(\beta-0-4)(\%)=\frac{\mathrm{I}(\mathrm{X})}{\mathrm{I}\left(\mathrm{C}_{9}\right)} \times 100 \\
& \mathrm{I}\left(\mathrm{C}_{9}\right)(\%)=0.5 \mathrm{I}\left(\mathrm{S}_{2,6}\right)+\mathrm{I}\left(\mathrm{G}_{2}\right)
\end{aligned}
$$

Where $\mathrm{I}\left(\mathrm{S}_{2,6}\right)$ is the integral value of $\mathrm{S}_{2,6}$, including $\mathrm{S}$ and $\left.\mathrm{S}^{\prime}, \mathrm{I}_{\left(\mathrm{G}_{2}\right)}\right)$ is the integration of $\mathrm{G}_{2}$, and $\mathrm{I}\left(\mathrm{C}_{9}\right)$ and $\mathrm{I}(\mathrm{X})$ represent the integral values of aromatic ring and $\alpha$ position of $\beta-\mathrm{O}-4$, respectively. 
5.3. Results and discussion

\subsubsection{Comparison of pretreatment effectiveness of ChCl-based solvents}

$\mathrm{ChCl}$ is the most common HBD used in the synthesis of DES due to its biodegradability and mass production. $\mathrm{ChCl}$ solution was studied as a pretreatment solvent in the present work. Compared with dilute acid pretreatment (corresponding to the amount of acid added to $75 \% \mathrm{ChCl}$ solution), acidified $\mathrm{ChCl}$ solution showed twice more xylan removal and five times more lignin removal, but less glucan loss (Table 1). As a result of substantial removal of non-cellulosic components and minor removal of cellulose (only $2.86 \%$ ), the pretreated switchgrass had an enriched cellulose content (55.95\%) (Table 5.1). Non-acidified $\mathrm{ChCl}$ solution had a much poorer performance, only removing $17.59 \%$ lignin but almost no xylan (Table 1). These results suggested strong synergism between acid and $\mathrm{ChCl}$ solution. In other words, the addition to $\mathrm{ChCl}$ led to increased proton reactivity and availability, which in turn promoted acid-catalyzed biomass fractionation and conversion reactions (Zhao \& Liu, 2013). The underlining mechanism of such accelerated reaction rates by $\mathrm{ChCl}$ is unclear. One possible explanation is that $\mathrm{ChCl}$ affects proton stabilization in water, a solvent effect similar to polar aprotic solvent (Mellmer et al., 2014). In addition, due to the cleavage of lignin-carbohydrate complex, more hemicellulose is exposed to acidic proton, which in turn facilitates hemicellulose hydrolysis. 
Table 5.1 Compositional change of switchgrass in response to the pretreatments using different solvents

\begin{tabular}{|c|c|c|c|c|c|c|c|c|}
\hline \multirow{2}{*}{ Pretreatment solvent } & \multirow{2}{*}{$\mathrm{pH}$} & \multirow{2}{*}{$\begin{array}{c}\text { Solid } \\
\text { recovery }(\%)\end{array}$} & \multicolumn{3}{|c|}{ Composition (\%) } & \multicolumn{3}{|c|}{ Removal (\%) } \\
\hline & & & Glucan & Xylan & Lignin & Glucan & Xylan & Lignin \\
\hline $1.0 \% \mathrm{H}_{2} \mathrm{SO}_{4}$ & 0.74 & $79.46 \pm 0.07$ & $37.90 \pm 0.75$ & $18.58 \pm 0.54$ & $22.99 \pm 0.29$ & $4.60 \pm 1.80$ & $22.75 \pm 2.19$ & $10.25 \pm 0.92$ \\
\hline $75 \% \mathrm{ChCl}$ & 5.20 & $91.50 \pm 0.88$ & $33.33 \pm 0.87$ & $20.88 \pm 0.17$ & $18.34 \pm 0.71$ & $3.43 \pm 1.58$ & $0.05 \pm 0.16$ & $17.59 \pm 2.40$ \\
\hline $75 \% \mathrm{ChCl}+1.0 \% \mathrm{H}_{2} \mathrm{SO}_{4}$ & 0.11 & $54.83 \pm 0.22$ & $55.95 \pm 0.24$ & $8.30 \pm 0.06$ & $18.15 \pm 0.29$ & $2.86 \pm 0.03$ & $76.17 \pm 0.08$ & $51.10 \pm 0.57$ \\
\hline
\end{tabular}


Previous studies have demonstrated acidic DESs were usually more effective than non-acidic DESs for removing lignin and xylan (Alvarez-Vasco et al., 2016; Xu et al., 2016). Such acidic DESs generally comprise $\mathrm{ChCl}$ and carboxylic acids (e.g., formic acid, lactic acid) and require relatively severe conditions (at least hours at temperature at $130{ }^{\circ} \mathrm{C}$ or above). Our study revealed that acidified $\mathrm{ChCl}$ solution was an effective pretreatment solvent at milder pretreatment conditions. Taken together, $\mathrm{ChCl}$ could facilitate the dissociation of lignocellulose complex by various Brønsted acids. From the economic perspective, $\mathrm{ChCl}$ solution supplemented with a small amount of cheap inorganic acid had a much lower cost than a DES comprising of $\mathrm{ChCl}$ and carboxylic acid. On the other hand, $\mathrm{ChCl}$ promoted the performance of dilute acid pretreatment while lowering its pretreatment severity.

\subsubsection{Recyclability and reusability of acidified $\mathrm{ChCl}$ solution}

Recycling a pretreatment solvent to a new pretreatment cycle can save pretreatment cost. Thus, $\mathrm{ChCl}$ solution was tested for its recyclability and reusability. The effects of repeatedly recycled solvent on biomass fractionation are shown in Table 5.2. For the first cycle of pretreatment using the original solvent, more than $70 \%$ xylan and 50\% lignin were removed while $97 \%$ glucan was preserved. Comparable xylan and lignin removal was achieved from the second cycle using the recycled solvent. However, the third cycle was much less effective than the previous two cycles, resulting in a drastic decrease in xylan and lignin removal. It was mainly due to the acid consumption during pretreatment, which was also reflected by an increase in $\mathrm{pH}$ from 0.11 to 1.25 . Acid was thus added to the fourth cycle to adjust its initial $\mathrm{pH}$ to the level for the $1^{\text {st }}$ cycle $(0.11)$, which boosted xylan removal and lignin removal (Table 5.2), achieving similar effectiveness to the first two cycles. These results confirmed that acidic condition is critical to lignocellulose fractionation using 
$\mathrm{ChCl}$ solution as discussed previously. The recycled solvent also had a good performance in the fifth cycle of pretreatment, but was less effective than the previous ones, even though acid was also added to adjust its initial $\mathrm{pH}$ to the level for the 1 st cycle. The accumulated impurities in the recycled liquor/solvent, such as ligninderived products and sugar degradation products, could affect the pretreatment. Minor solvent loss (i.e., $\mathrm{ChCl}$ loss) should be inevitable upon recycling and solid/liquid separation, a common issue with most solvent recycling. For instance, $\mathrm{ChCl}$ could be removed along with precipitated lignin due to high affinity between lignin and $\mathrm{ChCl}$ (Guo et al., 2013; Kim et al., 2018), and then rinsed away during lignin washing step. Further study is needed to investigate possible solvent loss and its effect on the pretreatment. 
Table 5.2 Compositional changes of switchgrass in response to the recyclability of acidified $\mathrm{ChCl}$ solution

\begin{tabular}{|c|c|c|c|c|c|c|c|c|}
\hline \multirow{2}{*}{$\begin{array}{l}\text { Pretreatment } \\
\text { Cycle }\end{array}$} & \multirow{2}{*}{ Sample Code } & \multirow{2}{*}{$\begin{array}{l}\text { Solid recovery } \\
(\%)\end{array}$} & \multicolumn{3}{|c|}{ Composition (\%) } & \multicolumn{3}{|c|}{ Removal (\%) } \\
\hline & & & Glucan & Xylan & Lignin & Glucan & Xylan & Lignin \\
\hline 1 & CL-1 & $54.83 \pm 0.22$ & $55.95 \pm 0.24$ & $8.30 \pm 0.06$ & $18.15 \pm 0.29$ & $2.86 \pm 0.03$ & $76.17 \pm 0.08$ & $51.10 \pm 0.57$ \\
\hline 2 & CL-2 & $55.75 \pm 0.46$ & $53.33 \pm 0.00$ & $10.05 \pm 0.20$ & $20.03 \pm .07$ & $5.85 \pm 0.77$ & $70.67 \pm 0.82$ & $45.14 \pm 0.25$ \\
\hline 3 & CL-3 & $63.70 \pm 1.25$ & $47.35 \pm 0.94$ & $13.72 \pm 0.98$ & $20.73 \pm 0.52$ & $4.52 \pm 0.02$ & $54.19 \pm 4.16$ & $35.07 \pm 2.89$ \\
\hline $4^{\mathrm{a}}$ & CL-4 & $54.51 \pm 0.06$ & $55.03 \pm 0.29$ & $8.67 \pm 0.12$ & $20.06 \pm 0.27$ & $5.01 \pm 0.39$ & $75.27 \pm 0.33$ & $46.26 \pm 0.66$ \\
\hline $5^{\mathrm{a}}$ & CL-5 & $58.35 \pm 0.89$ & $51.54 \pm 0.46$ & $9.22 \pm 0.75$ & $21.17 \pm 0.63$ & $4.77 \pm 2.31$ & $71.90 \pm 1.87$ & $39.33 \pm 0.87$ \\
\hline
\end{tabular}

${ }^{\mathrm{a}} \mathrm{H}_{2} \mathrm{SO}_{4}$ was added at $4^{\text {th }}$ and $5^{\text {th }}$ cycles to offset the acid consumed in the previous cycle. 
The pretreated switchgrass from different cycles was further characterized using XRD and FTIR. All the pretreated switchgrass showed an increased CrI ranging from 57 to 64 compared to untreated one (Figure 5.2). The increases in CrI could be primarily due to the efficient removal of lignin and xylan from switchgrass. FTIR results revealed the significant changes in the surface functional groups of switchgrass during the pretreatment (Figure 5.3). Several prominent peaks at $900 \mathrm{~cm}^{-1}(\mathrm{C}-\mathrm{O}-\mathrm{C}$ stretching in amorphous cellulose), $1103 \mathrm{~cm}^{-1}$ (C-O vibration in crystalline cellulose), 1035 and $1056 \mathrm{~cm}^{-1}$ (C-O stretching of cellulose) are related to cellulose (da Silva et al., 2018; Li et al., 2010). These peaks showed increased intensity to some extent after pretreatment, corroborating cellulose enrichment by the pretreatment. The peaks at $1240 \mathrm{~cm}^{-1}$ (C-O vibration in syringyl ring of lignin) and $1510 \mathrm{~cm}^{-1}$ (aromatic skeletal vibration) are characteristic peaks for lignin (Dai et al., 2017). The intensity of these peaks reduced after the pretreatment, indicating lignin removal (Dai et al., 2017). The peaks at $1375 \mathrm{~cm}^{-1}$ and $1160 \mathrm{~cm}^{-1}$ correspond to $\mathrm{C}-\mathrm{H}$ deformations and $\mathrm{C}-\mathrm{O}-\mathrm{C}$ vibration, respectively, in cellulose and hemicellulose (Bekiaris et al., 2015). The peak at $1730 \mathrm{~cm}^{-1}$ is assigned to unconjugated $\mathrm{C}=\mathrm{O}$ in xylan. The pretreatment appeared to weaken the peak intensity at 1375 and $1730 \mathrm{~cm}^{-1}$, which could be attributed to hemicellulose removal. The relative intensity of lignin reference peak at $1510 \mathrm{~cm}^{-1}$ against cellulose reference peak at $900 \mathrm{~cm}^{-1}$ is indicative of relative change in lignin/cellulose content of biomass (Lupoi et al., 2014; Pandey \& Pitman, 2004; Pandey \& Pitman, 2003). As shown in Table 5.3, $\mathrm{I}_{1510} / \mathrm{I}_{900}$ decreased after the pretreatment, corroborating substantial delignification by acidified $\mathrm{ChCl}$ solution. Both XRD and FTIR results were consistent with those revealed by compositional analysis. 


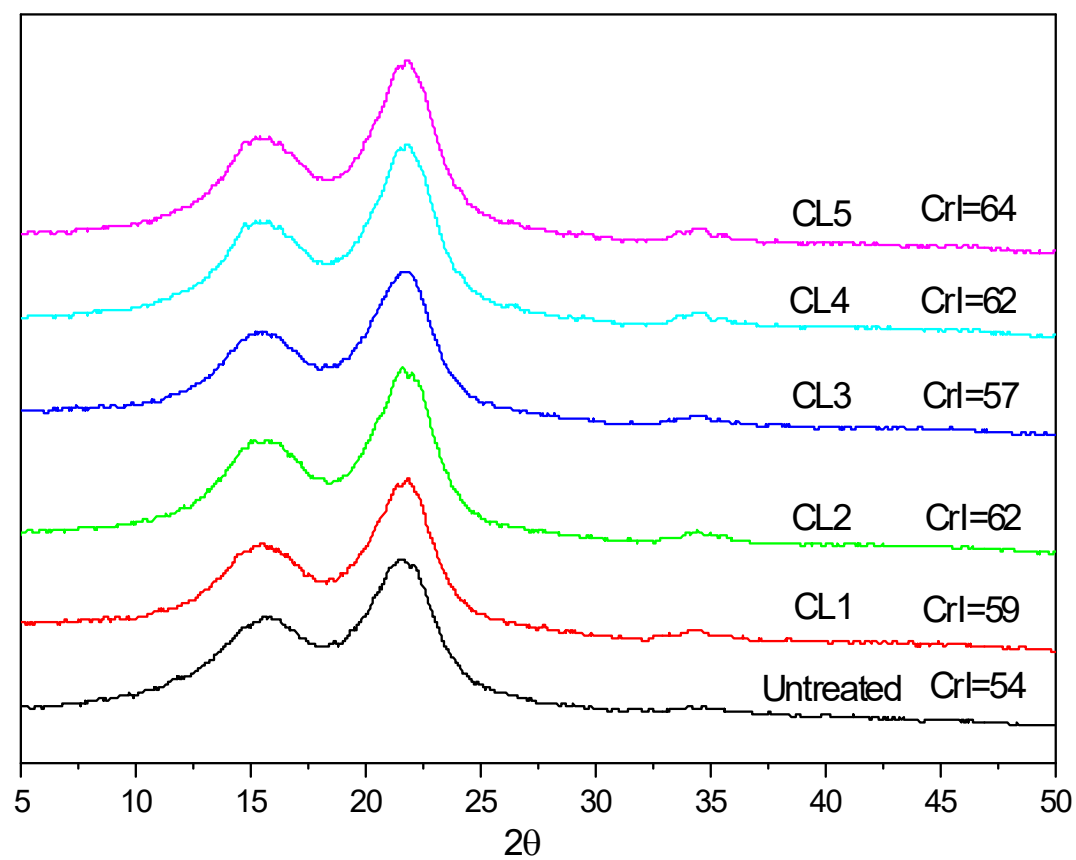

Figure 5.2 XRD pattern of the pretreated switchgrass from different pretreatment cycle.

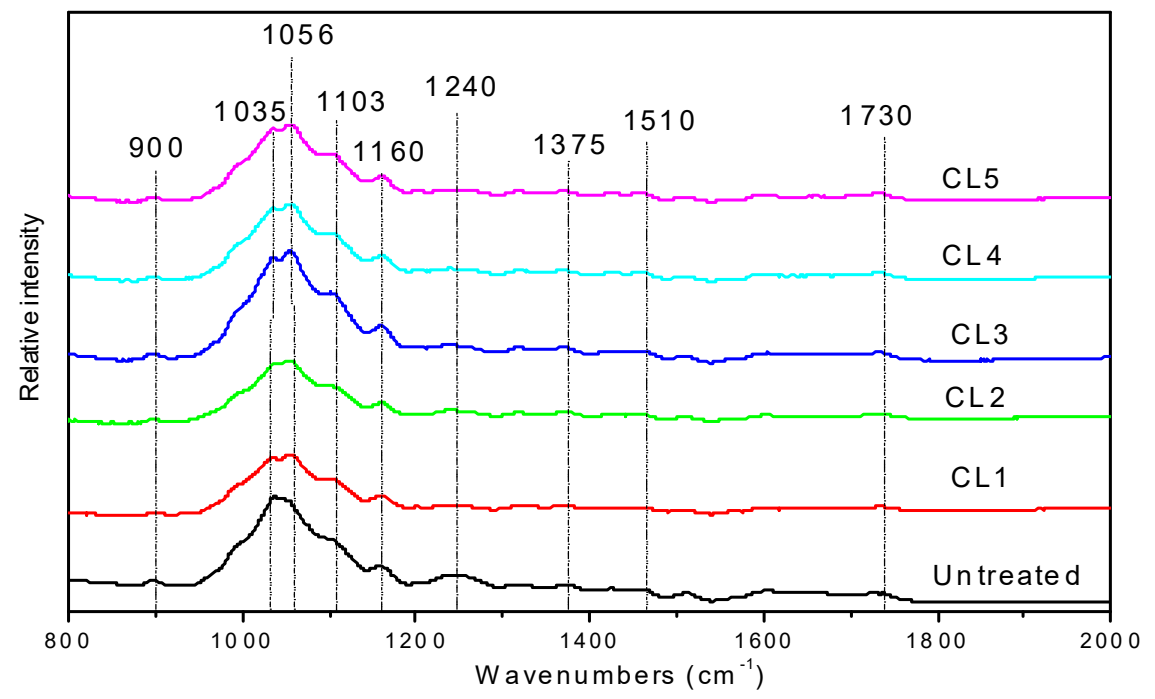

Figure 5.3 FTIR spectra of pretreated switchgrass from different pretreatment cycles. 
Table 5.3 Relative peak intensity of lignin and carbohydrates

\begin{tabular}{cc}
\hline Sample code $^{\mathrm{a}}$ & $\mathrm{I}_{1510} / \mathrm{I}_{900}{ }^{\mathrm{b}}$ \\
\hline Untreated & 1.31 \\
CL1 & 0.76 \\
CL2 & 0.80 \\
CL3 & 0.75 \\
CL4 & 1.00 \\
CL5 & 0.85 \\
\hline
\end{tabular}

${ }^{a}$ CL1-5: Pretreated switchgrass from the first to the fifth pretreatment cycle.

${ }^{\mathrm{b}} \mathrm{I}_{1510} / \mathrm{I}_{900}$ is expressed as peak height ratio.

\subsubsection{Digestibility of pretreated switchgrass}

Improving cellulose digestibility for sugar production is one major objective of biomass pretreatment. In this study, the pretreatment using acidified $\mathrm{ChCl}$ solution improved the glucose yields by up to 5 folds. Non-acidified $\mathrm{ChCl}$ solution had minor effects on the pretreatment and led to no improvement in the glucose yield. Dilute acid broke down switchgrass to some extent and doubled its cellulose digestibility (Figure 5.4). However, such pretreatment performance was far behind that of $\mathrm{ChCl}$ containing dilute acid solution (i.e., acidified $\mathrm{ChCl}$ solution) (Figure 5.4). The glucose yield as high as $70 \%$ was observed with switchgrass pretreated with acidified $\mathrm{ChCl}$ solution under non-optimized condition. Great improvement in glucose yield was primarily attributed to significant removal of xylan and lignin by acidified $\mathrm{ChCl}$ solution, which allowed cellulase to have a better access to cellulose. The pretreated switchgrass from the second cycle showed similar digestibility to the first one (Figure 5.5). The recycled solvent also had good performance on the third cycle, but was not as effective as the previous two cycles since glucose yield dropped to $60 \%$ (Figure 5.5). As aforementioned, acid consumption at the first two cycles led to reduced performance of acidified $\mathrm{ChCl}$ solution. Supplementing acid to the recycled solvent at the fourth cycle thus boosted its capability. The glucose yield was near $70 \%$, similar to those resulting from the first two cycles (Figure 5.5). However, even with acid 
supplemented, the fifth cycle yielded the least digestible cellulose pulp than the previous one. Most likely, a higher lignin content affected enzymatic hydrolysis efficiency due to non-productive binding of hydrolytic enzymes with lignin (Rahikainen et al., 2013). It should be noted that that xylan remaining after the acidified $\mathrm{ChCl}$ solution pretreatment appeared to be more digestible than cellulose when its content was relatively low (10\% or lower). Higher xylose yields than glucose yields were also observed with alkaline pretreated spruce and dilute acid pretreated biomass sorghum (Dien et al., 2009; Zhao et al., 2008). Since biomass digestibility is feedstock- and pretreatment-dependent, it seems to be difficult to find a clear trend of the disparity in polysaccharide digestibility. Nonetheless, the pretreated solids from all the pretreatment cycles in this study yielded more than $40 \%$ sugars compared to untreated one. Effective lignin and xylan removal as well as good digestibility of pretreated biomass across all the pretreatment cycles demonstrated the feasibility of reusing $\mathrm{ChCl}$ solution for biomass pretreatment.

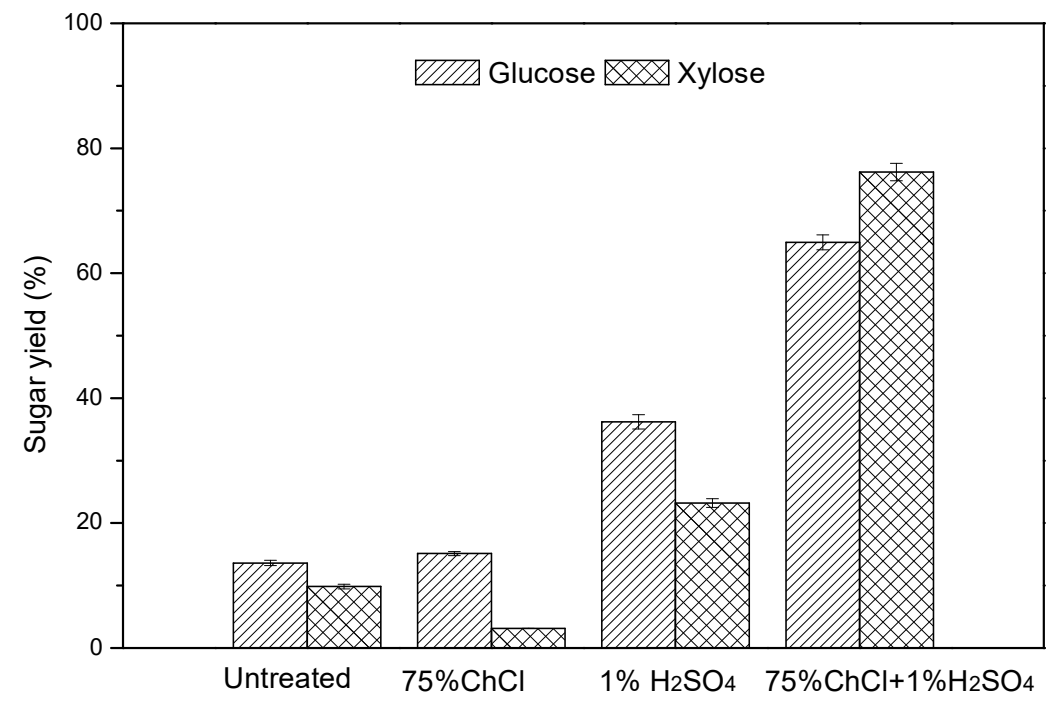

Figure 5.4 Digestibility of switchgrass pretreated by different solvents. 


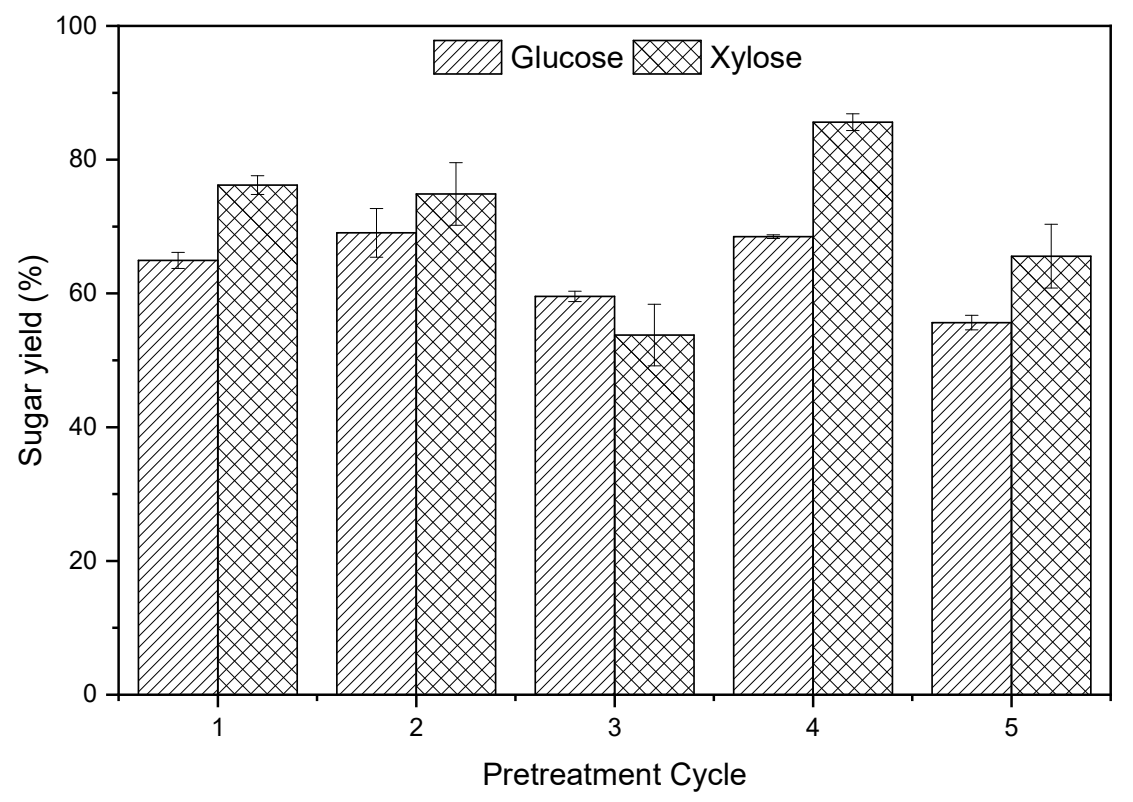

Figure 5.5 Digestibility of the pretreated switchgrass from different pretreatment cycles.

\subsubsection{Lignin recovery}

"Lignin-first" pretreatment also targets maximizing lignin yield along with pulp digestibility. Lignin yield for each cycle was presented in Figure 5.6. For the first cycle using the fresh solvent, lignin recovered from the pretreatment liquor accounted for $50 \%$ of lignin removed during the pretreatment. A gradual increase in lignin yield was observed for the following cycles, and the yields exceeded $100 \%$ during the fourth and fifth pretreatment cycles. A similar trend was also found with a recyclable aqueous ionic liquid made from triethylamine and $\mathrm{H}_{2} \mathrm{SO}_{4}$ (Brandt-Talbot et al., 2017). Such phenomenon was due to the co-precipitation of "old" lignin carried from previous cycles and "new" lignin in a new pretreatment cycle. The co-precipitation of the "old" and "new" lignin could avoid the build-up of lignin in the liquor (BrandtTalbot et al., 2017). As a result, total lignin recovery (cumulative lignin yield) reached $93 \%$ at the fifth cycle (Figure 5.6). It remains unclear what promoted lignin coprecipitation and what distribution recovered lignin had. In addition to real lignin, 
recovered lignin may also contain pseudo-lignin since acidic pretreatment condition favors its formation (Sannigrahi et al., 2011). However, pseudo-lignin cannot be distinguished from real lignin based on compositional analysis. Comprehensive characterization techniques, including scanning electron microscopy, FTIR, and NMR, are necessary to qualify and quantify pseudo-lignin (Sannigrahi et al., 2011).

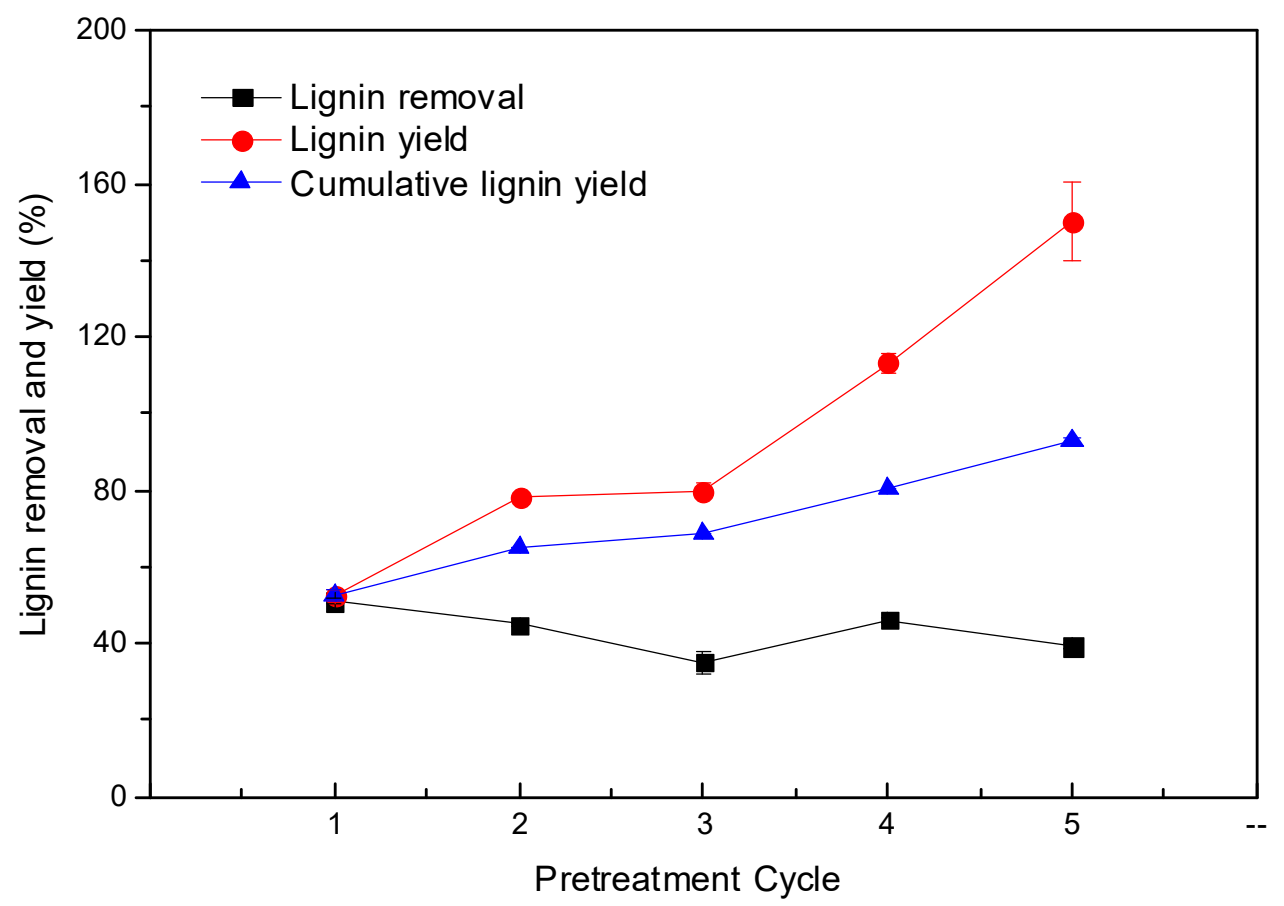

Figure 5.6 Lignin removal and yield resulting from all the pretreatment cycles.

\subsubsection{Properties of recovered lignin}

To better understand the structural transformation of lignin during the pretreatment, 2D HSQC NMR was used to analyze lignin. Native lignin (NL) and lignin from the first (L-1) and fifth (L-5) cycles were analyzed, and main ${ }^{13} \mathrm{C}-{ }^{1} \mathrm{H}$ cross-signals in the HSQC spectra of switchgrass lignin were assigned according to previous studies (Figure 5.7) (Samuel et al., 2011; Samuel et al., 2010). The presence of $\beta-\mathrm{O}-4$ substructure (A) in NL, L-1, and L-5 was detected by the cross peaks at $\delta_{\mathrm{C}} / \delta_{\mathrm{H}} 71.84 / 4.86\left(\mathrm{~A}_{\alpha}\right), 83.68 / 4.29\left(\mathrm{~A}_{\beta}\right)$, and 60.00/3.60 $\left(\mathrm{A}_{\gamma}\right)$ (Figure 5.7). Semi- 
quantification of $\beta-\mathrm{O}-4$ linkage revealed that its percentages were $44 \%$ and $42 \%$ for NL and L-5, respectively, while $33 \%$ for L-1. It is unclear what was primarily responsible for such a difference. One possible explanation was a higher water content of the recycled solvent in the fifth cycle due to $\mathrm{ChCl}$ loss upon solvent recycling as aforementioned, which could provide more protection for $\beta-\mathrm{O}-4$ linkage. Further study is needed to systematically investigate the effects of water in $\mathrm{ChCl}$ solution on lignin chemistry. Compared to the complete cleavage of $\beta-\mathrm{O}-4$ linkage in lignin by acidic ChCl-based DESs (e.g., ChCl:LA) (Alvarez-Vasco et al., 2016), acidified $\mathrm{ChCl}$ solution was shown to well preserve $\beta$-O-4 linkage in lignin. This finding is particularly important to lignin monomer production because theoretical lignin monomer yields was reported to be roughly proportional to the square of percentage of cleavable inter-unit ether bonds (i.e., $\beta$-O-4) (Renders et al., 2017). While an exciting example was reported for stabilizing cleavable ether bonds in lignin by adding formaldehyde to pretreatment (Shuai et al., 2016a), there was a tradeoff between lignin stabilization and cellulose digestibility. This present work overcame such a challenge by using an innovative $\mathrm{ChCl}$ solution, realizing both high cellulose digestibility and well-preserved ether bonds in lignin. It is worth evaluating actual monomer yields of such technical lignin in the future study.

Major cross signals in the aromatic region corresponded to aromatic rings of lignin units (Figure 5.7). Syringyl (S) and guaiacyl (G) units were clearly separated. S units showed a prominent correlation for $\mathrm{C}_{2,6}-\mathrm{H}_{2,6}$ at $\delta_{\mathrm{C}} / \delta_{\mathrm{H}} 103.93 / 6.71\left(\mathrm{~S}_{2,6}\right)$, while $\mathrm{G}$ units showed the correlations at $111.27 / 6.99\left(\mathrm{G}_{2}\right), 115.58 / 6.78\left(\mathrm{G}_{5}\right)$, and 119.00/6.82 $\left(\mathrm{G}_{6}\right)$. The correlation for $\mathrm{C}_{2,6} / \mathrm{H}_{2,6}$ in $p$-hydroxyphenyl units $(\mathrm{H})$ was also observed at 129.96/7.53 $\left(\mathrm{H}_{2,6}\right)$. Cinnamate unit arising from $p$-coumarat $(p$-CA) and ferulate (FA) was clearly detected in NL, but shrank in L-1 and L-5. In grass, cinnamate units link 
to lignin and hemicellulose, forming ether and ester linkages, respectively, which are responsible for cell wall recalcitrance (Brandt et al., 2015; Rodriguez et al., 2017). The reduction of cinnamate unit after pretreatment implied that acidified $\mathrm{ChCl}$ solution solubilized lignin and hemicellulose by cleaving their cross-links with ferulate.
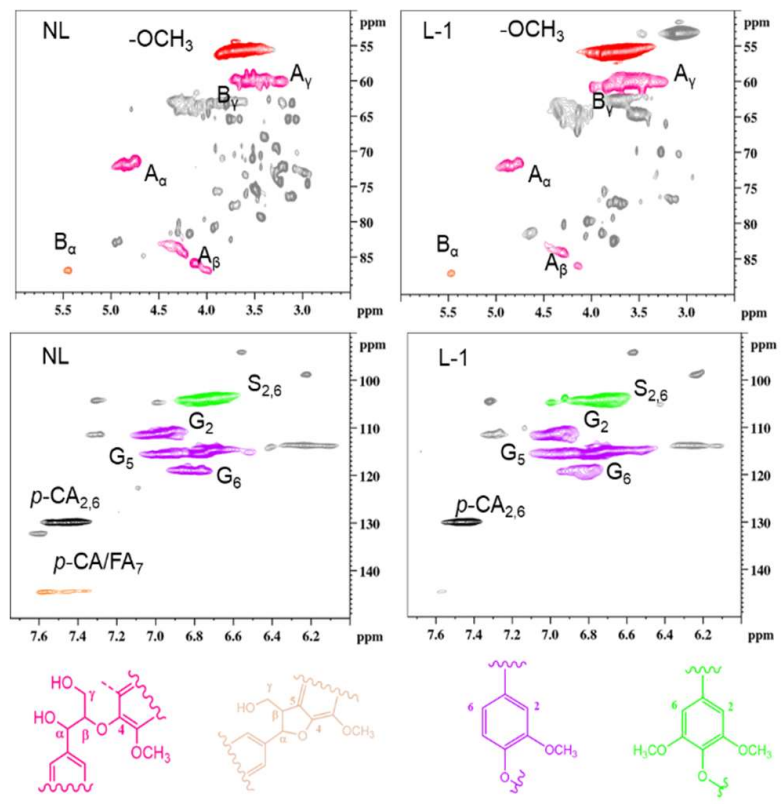

$\mathrm{A}(\beta-\mathrm{O}-4)$
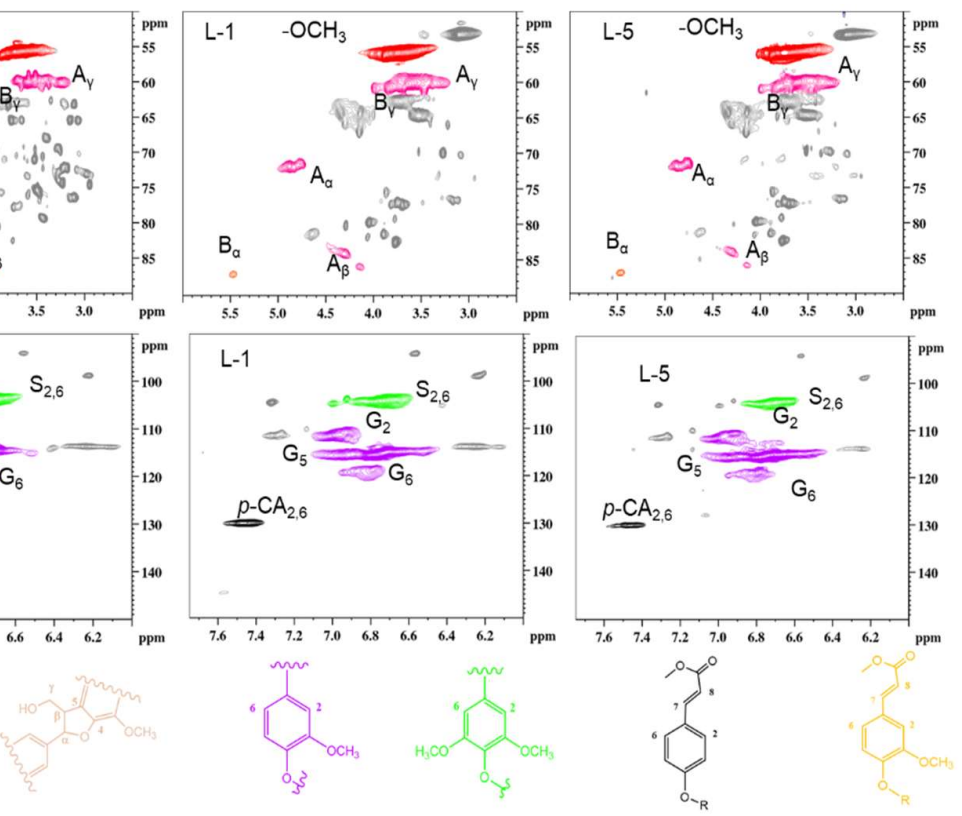

$B(\beta-5)$

G(Guaiacyl unit) S(Syringyl unit)

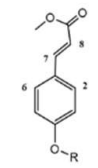

$p$-CA(p-Coumarate) $\quad \mathrm{FA}($ Ferulate $)$

Figure 5.7 2D HSQC NMR spectra of lignin

\subsubsection{Xylose-rich pretreatment liquor used for furfural production}

In addition to digestible solids and lignin, xylose-rich pretreatment liquor was another stream resulting from pretreatment in this study. Since the pretreatment liquor was repeatedly recycled and reused as a pretreatment solvent, xylose was accumulated to a substantial amount at the final cycle. HPLC results revealed that the liquor collected from the final pretreatment cycle contained $45.5 \mathrm{~g} / \mathrm{L}$ xylose (data not shown), which corresponded to $60.2 \%$ of total xylan removed/solubilized from all the five pretreatment cycles. In addition to being hydrolyzed into xylose, xylan can also 
be degraded into sugar degradation byproducts, react with lignin degradation products to form pseudo-lignin, and depolymerized into xylo-oligasacchrides. The xylose-rich pretreatment liquor can be a good substrate for furfural production. In addition, the liquor was an acidic $\mathrm{ChCl}$ solution, which could be a suitable reaction medium for catalytic upgrading. Thus, it was worth valorizing the pretreatment liquor into furfural while minimizing the generation of waste stream.

Temperature is a key factor influencing the furfural production from xylose. Its effect on the furfural formation in acidified $\mathrm{ChCl}$ solution was examined using $50 \mathrm{~g} / \mathrm{L}$ xylose. As shown in Figure 5.8, furfural production almost tripled when temperature was increased from 130 to $160^{\circ} \mathrm{C}$, reaching $16.1 \mathrm{~g} / \mathrm{L}$ furfural with $50 \%$ yield. Prior studies also reported that high temperature is favorable to furfural formation (Marcotullio \& De Jong, 2010; Yemiş \& Mazza, 2011). According to Loow et al. (Loow et al., 2018), an increase in reaction severity, such as higher temperatures and longer reaction time, in a DES environment appeared to favor the dehydration of carbohydrates to furanic derivatives. As the pretreatment liquor contained impurities, further increasing the temperature may lead to undesired side reactions. The reaction temperature of $160^{\circ} \mathrm{C}$ was thus selected for the other tests. Similar to reaction temperature, reaction time also had significant effects on furfural production. Increasing the reaction time from 30 to $50 \mathrm{~min}$ led to an increase in furfural titer from 9.7 to $16.1 \mathrm{~g} / \mathrm{L}$, but further increasing the time to $80 \mathrm{~min}$ led to marginal improvement in furfural production (Figure 5.9). 


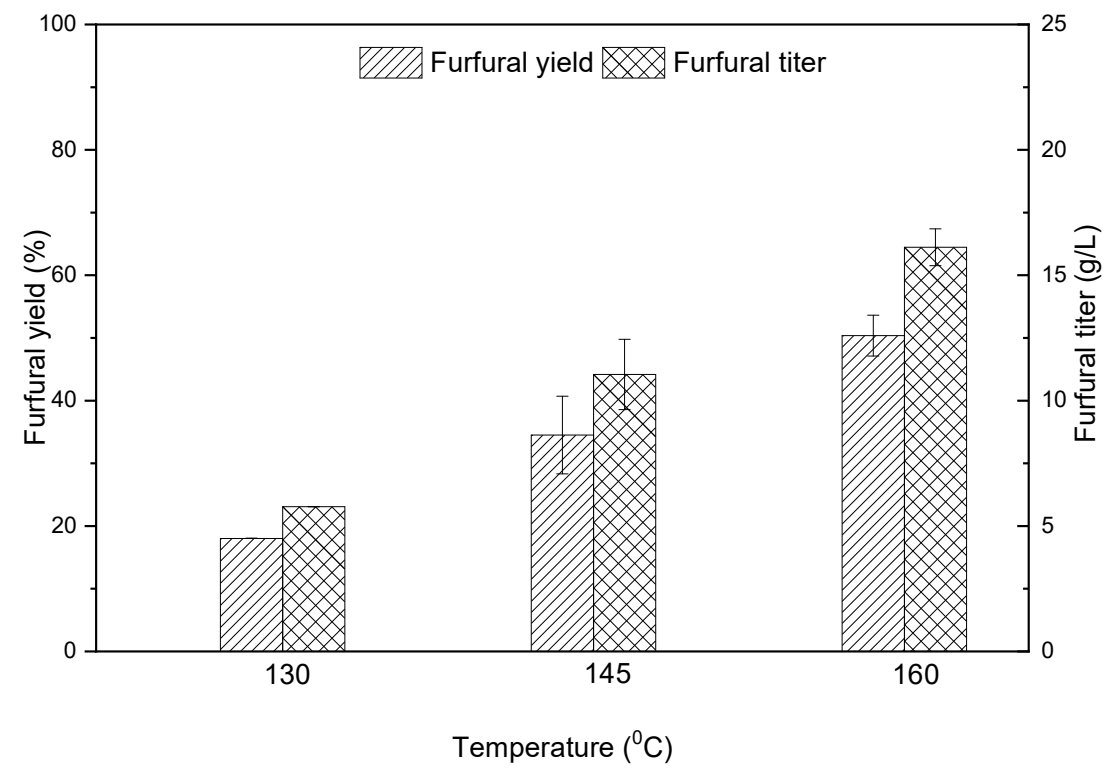

Figure 5.8 Effects of reaction temperature on furfural production from xylose in acidified $\mathrm{ChCl}$ solution.

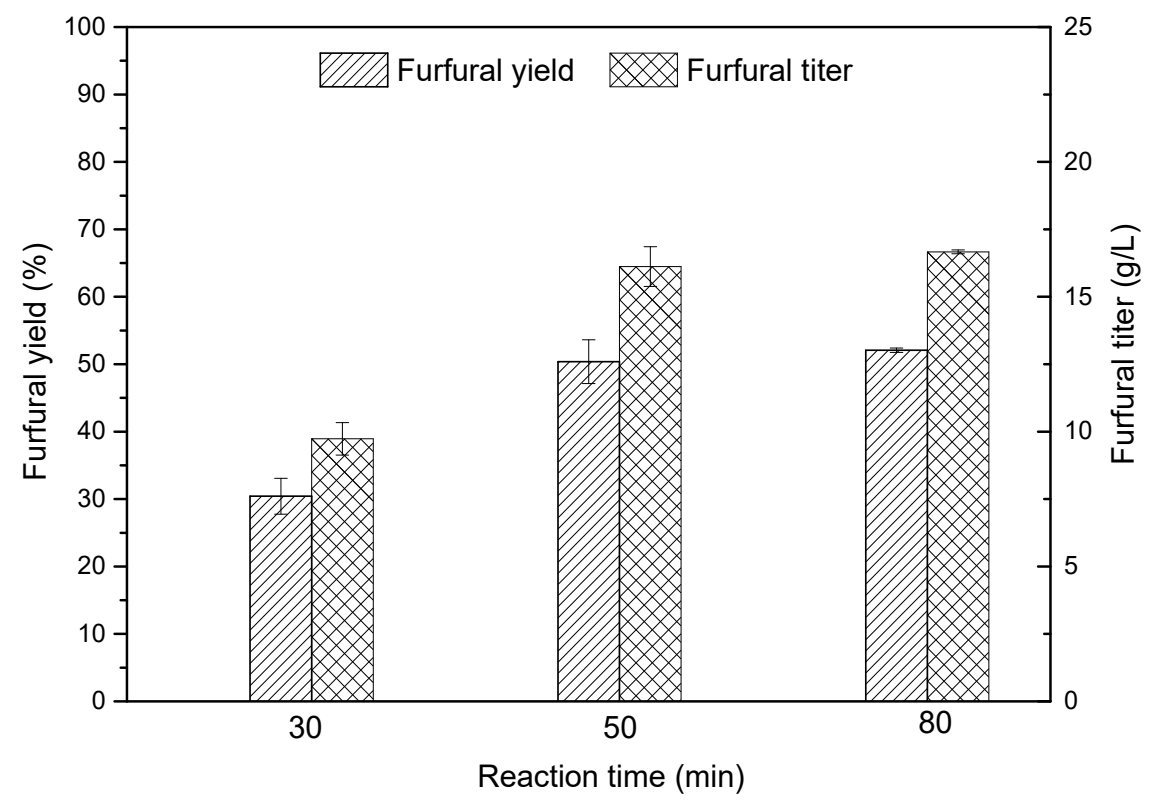

Figure 5.9 Effects of reaction time on furfural production from xylose in acidified $\mathrm{ChCl}$ solution. 
Metal chloride salts can enhance the formation of 1,2-enediol from the acyclic form of the aldose, the precursor of furfural, under acidic condition (Marcotullio \& De Jong, 2010). $\mathrm{AlCl}_{3}$ has been shown to be a good catalyst for improving furfural production from xylose/xylan in acidic ChCl-based DESs (Cai et al., 2014; Zhang \& $\mathrm{Yu}, 2013)$. In this study, $\mathrm{AlCl}_{3}$ was found to not only enhance the furfural yield, but also accelerate the reaction in acidified $\mathrm{ChCl}$ solution. As shown in Figure 5.10, $\mathrm{AlCl}_{3}$ addition at $2 \%(\mathrm{w} / \mathrm{v})$ led to $4 \%$ more furfural formed when the liquor was reacted for $50 \mathrm{~min}$. The beneficial effects of $\mathrm{AlCl}_{3}$ became more obvious when the reaction time was shorter. A furfural yield of $60 \%$ was obtained from 15 min reaction with $2 \%$ (w/v) $\mathrm{AlCl}_{3}$ addition. Depending on reaction media and conditions, $\mathrm{AlCl}_{3}$ added to furfural production from xylose ranges from 1.2 \% to 5.3\% (Cai et al., 2014; Lopes et al., 2017; Zhang et al., 2013). High $\mathrm{AlCl}_{3}$ loading may not necessarily increase furfural yield considering both accelerated furfural production and side reactions (e.g., condensation reactions, furfural degradation) (Cai et al., 2014). Although it is beyond the scope of the present work, it is necessary in the future study to optimize $\mathrm{AlCl}_{3}$ loading and explore other strategies in order to maximize furfural production (Cai et al., 2014). 


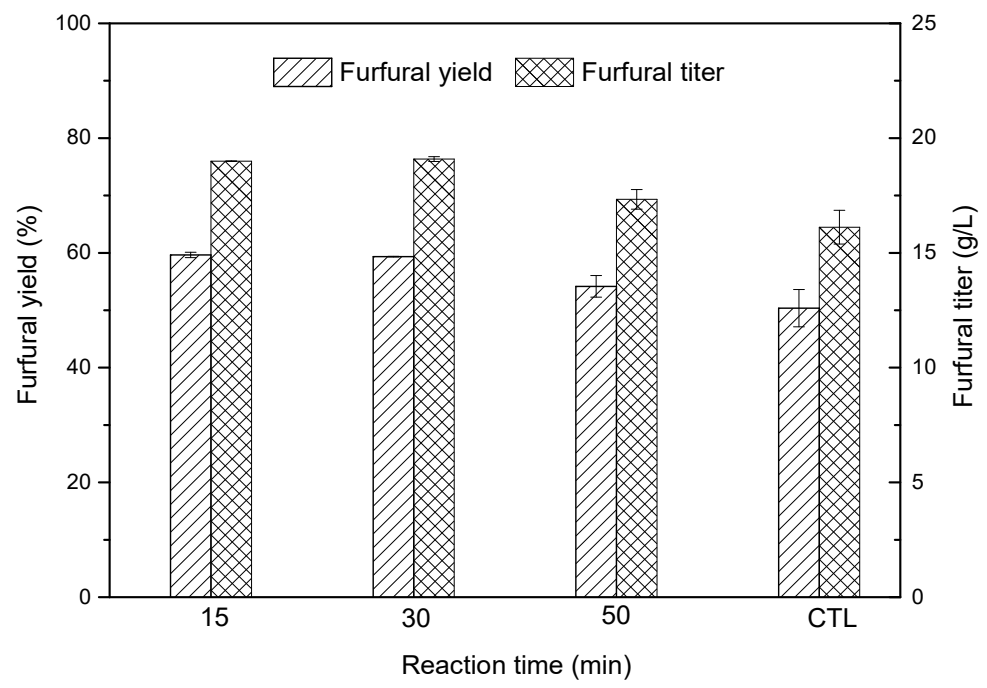

Figure 5.10 Effects of $\mathrm{AlCl} 3$ on furfural production from xylose in acidified $\mathrm{ChCl}$ solution.

Furfural production from the pretreatment liquor was evaluated using the above optimized condition (i.e., $160{ }^{\circ} \mathrm{C}, 15 \mathrm{~min}, 2 \% \mathrm{w} / \mathrm{v} \mathrm{AlCl}_{3}$ ). Compared to xylosecontaining $\mathrm{ChCl}$ solution, the liquor gave a reduced furfural titer $(14.12 \mathrm{~g} / \mathrm{L})$ and yield (48.22\%) (data not shown). It could be largely due to the presence of impurities in the liquor, which can interfere with xylose-to-furfural reactions. Lignin and ligninderived compounds are typical impurities present in pretreatment liquor. In the tested pretreatment liquor, there was about $7 \%$ lignin remaining. Intermediate compounds derived from xylose, such as dehydrofuranose sugar, could react with lignin via aromatic substitution reactions, which in turn decreased furfural selectivity (Dussan et al., 2016), Aldol condensation between carbonyl groups in lignin and furfural may also lead to furfural degradation (Dussan et al., 2016). Further investigation is required to elucidate the exact mechanism of such side reactions in the presence of lignin. Conditioning pretreatment liquor would make such a "waste" stream more favorable for value-added upgrading. 


\subsection{Conclusion}

Acidified $\mathrm{ChCl}$ solution was capable of efficiently solubilizing lignin and xylan from switchgrass under a relatively mild condition $\left(120^{\circ} \mathrm{C}, 25 \mathrm{~min}\right)$. Lignin can be recovered by simple precipitation, showing well preserved $\beta-\mathrm{O}-4$ linkages. The pretreatment improved the cellulose digestibility of switchgrass by about 5 folds. The solvent can be recycled for at least four times with good pretreatment performance. Solvent recycling also led to $93 \%$ total lignin recovery and xylose enrichment in the pretreatment liquor. The resultant xylose-enriched liquor was proved to be a good substrate and reaction medium for furfural production, yielding $14.12 \mathrm{~g} / \mathrm{L}$ furfural with a yield of $48.22 \%$. These findings indicated that bio-based $\mathrm{ChCl}$ solution was an effective, recyclable solvent for lignocellulose pretreatment and lignin extraction as well as furfural production. 


\section{Chapter 6 Aqueous Choline Chloride Enables Biomass Fractionation and Conversion in One-pot}

\subsection{Introduction}

Lignocellulosic biomass renders modern society a sustainable feedstock to produce bio-based chemicals and biofuels, which help to mitigate the problems stemming from utilization of fossil fuels, such as climate change and environmental pollution. Many research efforts have been devoted to the valorization of lignocellulosic biomass into initial platform chemicals which can be further processed into a wide range of valuable products (Araji et al., 2017; Mika et al., 2017). Furfural, as one of the platform chemicals, has garnered great interests as it can be used for manufacturing a wide range of furan-based chemicals and solvents, and its market demand continues to grow (Cai et al., 2014). Industrially, furfural can produced from xylan-rich lignocellulosic materials with mineral acids as catalyst (Peleteiro et al., 2016). In the presence of catalyst, hemicellulose is hydrolyzed into pentose, which undergoes dehydration to form furfural with a yield between 40-50\% (Mamman et al., 2008). While hemicellulose can be upgraded into value-added furfural, the furfural residue, which is mainly composed of lignin and cellulose, is often treated as a waste and is simply incinerated for steam generation (Mamman et al., 2008). It is estimated that China, the largest producer of furfural, gives an annual production of 23 million tons of furfural residue (Chen et al., 2014; Mamman et al., 2008).

Co-production of other value added products from the furfural residue can increase the revenue for the furfural industry and also eliminate waste discharge (Cai et al., 2014; Moghaddam et al., 2017). One popular pathway for cellulose utilization is 
to enzymatically hydrolyze it into glucose, which can be converted to biofuels and chemicals, such as ethanol and butanol (Chen et al., 2014; Kai et al., 2016). However, the process adopted industrially for furfural manufacturing has a low selectivity for lignin solubilization, which often results in a high lignin content in the furfural residual. As the lignin is a major barrier blocking cellulase access to cellulose and deactivate the cellulase via non-productive binding, the furfural residue with a high lignin content often experiences a low to moderate sugar yield (Kai et al., 2016). Pretreatment, such as formic acid fractionation, alkaline extraction and ethanolsolv, has been proposed to effectively remove lignin from the industrially furfural residue (Chen et al., 2014; Moghaddam et al., 2017; Yu et al., 2014). Such pretreatment not only improves the cellulose digestibility, but also enables extraction of quality lignin with less carbohydrate "contamination", which is beneficial to lignin valorization. However, additional steps for improving the valorization potential of furfural residue could increase the process cost, waste generation, and the make it less economically attractive. It is desirable to develop a process that enables hemicellulose conversion into furfural with high yield, while simultaneously produces lignin and cellulose streams amenable to further upgrading in s single step.

Deep eutectic solvents (DESs) are emerging solvents holding great promise for lignocellulosic biomass processing (Tang et al., 2017). The deep eutectic solvent formed from choline chloride $(\mathrm{ChCl})$ and carboxylic acids have been reported to efficiently fractionate lignocellulosic biomass into different streams (ie., cellulose, lignin, hemicellulose), and significantly improve the cellulose digestibility (Hou et al., 2017; Xu et al., 2016; Zhang et al., 2016a). ChCl based DESs can also be used as reaction medium for furfural production from xylose or xylan (Zhang et al., 2014). 
Our previous study also revealed that the acidified aqueous $\mathrm{ChCl}$ can efficiently solubilize more than $70 \%$ xylan and 50\% lignin from switchgrass at a mild condition, and the pretreatment liquor enriched with xylan-derived sugars can be upgraded into furfural (Chen et al., 2018b). Feasibly, this process can be further simplified by integrating lignin solubilization, furfural production, and digestible cellulose pulp production into one pot.

However, furfural, once formed, can be degraded via resinification and condensation reactions, which lead to the formation of humins (Mittal et al., 2017a). Bi-phasic system has been proposed as an alternative to traditional furfural production system by using an inert organic solvent to extract furfural and prevent its degradation (Zhang et al., 2017b). Methyl isobutyl ketone (MIBK) is one commonly used extraction solvent in a biphasic medium as it has a high partition coefficient with furfural and can be produced from biomass-derived acetone (Antonyraj et al., 2014; Mittal et al., 2017a).

In this study, a biphasic system consisted of acidified aqueous $\mathrm{ChCl} / \mathrm{MIBK}$ was used for efficient fractionation of switchgrass, and simultaneous production of furfural, cellulose pulp, and lignin. The recyclability of $\mathrm{ChCl}$ was examined, and the digestibility of cellulose residue was evaluated.

\subsection{Materials and methods}

\subsubsection{Feedstocks}

Switchgrass was collected from the Bradford Farm at the University of Missouri in Columbia, Missouri, USA. The raw switchgrass was air-dried and ground through 40 mesh screen, and stored in plastic bags. Hydrolytic enzymes (Cellic ${ }^{\circledR}$ CTec2) were 
kindly provided by Novozyme (Franklinton, NC, USA). All the chemicals were purchased from Fisher Scientific, and used without any further purification (Hampton, NH, USA).

\subsubsection{One-pot furfural production from switchgrass}

As shown by Figure 6.1, one-pot furfural production was conducted by loading $0.5 \mathrm{~g}$ switchgrass, $5 \mathrm{~g}$ acidified aqueous $\mathrm{ChCl}$ solution (70 wt $\%$ ), and $5 \mathrm{ml}$ MIBK into a glass pressure tube, unless otherwise stated. Then, the pressure tube was placed into a preheated oil bath for a certain time. Different pretreatment temperature, acid concentration, solid loading, and reaction time were examined as present in Table 1. Upon the end of reaction, the pressure tube was taken out immediately, and cooled down to room temperature. Then, the upper layer (MIBK) was decanted into a glass vial carefully, and the rest slurry was mixed with $10 \mathrm{~mL}$ acetone/water $(1: 1, \mathrm{v} / \mathrm{v})$. The mixture was filtered, and the retained residue was washed with another $10 \mathrm{~mL}$ acetone/water $(1: 1, \mathrm{v} / \mathrm{v})$ twice. The above filtrate was combined, and the acetone in the filtrate was removed under reduced pressure. Lignin was precipitated upon acetone removal and was collected by centrifugation at $12,000 \times \mathrm{g}$ for $5 \mathrm{~min}$, and washed using deionized water 4 times. The washed lignin was dried in an oven at $45^{\circ} \mathrm{C}$ for $12 \mathrm{~h}$, and then stored in sealed glass vial before further analysis. 


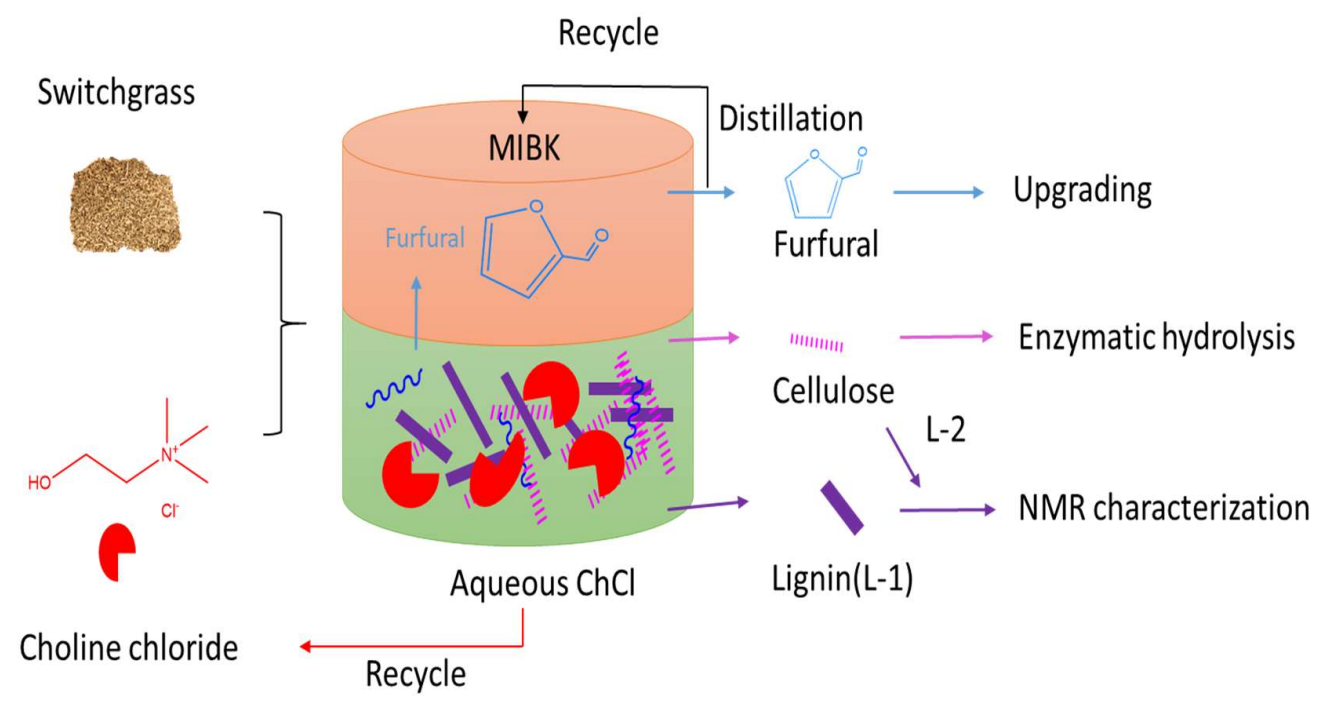

Figure 6.1 Schematic of the one-pot process

\subsubsection{One-pot furfural production using recycled solvent}

For aqueous $\mathrm{ChCl}$ recycling, the liquor after lignin removal was collected, and excessive water was evaporated in a convection oven at $70^{\circ} \mathrm{C}$. Due to the consumption of acid during each cycle, $0.2 \%(\mathrm{w} / \mathrm{w})$ acid was supplemented to the recycled $\mathrm{ChCl}$ solution to make its $\mathrm{pH}$ equal to the freshly prepared one. Then, onepot furfural production using recycled $\mathrm{ChCl}$ solution was conducted under optimized conditions from Section 6.2.2.

\subsubsection{Enzymatic hydrolysis}

Treated switchgrass from different cycles was loaded into a glass vial containing citrate buffer (50 mM, pH 5.5). Cellic ${ }^{\circledR}$ CTec2 was loaded at a dosage of $20 \mathrm{mg}$ protein/g solid. The total reaction volume was $5 \mathrm{ml}$, and the solid loading was $2 \%$ (w/w). After $48 \mathrm{~h}$, the hydrolysate was centrifuged at 17,000 $\times \mathrm{g}$ for $2 \mathrm{~min}$, and the supernatant was collected and stored at $-20^{\circ} \mathrm{C}$ before analysis. 


\subsubsection{Analytical methods}

Compositional analysis of untreated and pretreated biomass were conducted by using the standard method (Sluiter et al., 2008). Briefly, oven-dried samples were hydrolyzed with $72 \%$ sulfuric acid at $30{ }^{\circ} \mathrm{C}$ for $60 \mathrm{~min}$, followed by a second step hydrolysis with $4 \%$ sulfuric acid at $121^{\circ} \mathrm{C}$ for $60 \mathrm{~min}$. Acid insoluble lignin was determined gravimetrically, while acid soluble lignin was determined spectrophotometrically by measuring the filtrate absorbance at $320 \mathrm{~nm}$. Sugar monomers were analyzed by High Performance Liquid Chromatography (HPLC) Agilent 1200 series (Agilent Technologies, Inc., USA) equipped with a Refractive index detector and a HPX-87P $(300 \times 7.8 \mathrm{~mm})$ column $($ Bio-Rad, USA $)$. The RID and column temperature were maintained at 35 and $80{ }^{\circ} \mathrm{C}$ respectively, and the mobile phase was HPLC grade water, eluting at a flow rate of $0.6 \mathrm{ml} / \mathrm{min}$. Furfural concentration was determined by using the same HPLC system equipped with a $\mathrm{C}_{18}$ column (ZOBRAX), with $20 \mathrm{vol} \%$ methanol as mobile phase at a flow rate of 1 $\mathrm{ml} / \mathrm{min}$. The column and RID detector temperature was maintained at 30 and $35^{\circ} \mathrm{C}$ respectively. 2D HSQC NMR analysis of both recovered lignin and native lignin was conducted following the method described in our previous studies (Chen et al., 2018b).

\subsection{Results and discussion}

\subsubsection{Effects of reaction conditions on furfural production and compositional change}

To optimize the furfural production from switchgrass, parameters including temperature, acid concentration, solid loading, and reaction time were investigated (Figure 6.1). As the furfural partition coefficient in $\mathrm{MIBK}$ and aqueous $\mathrm{ChCl}$ was determined to be as high as 5.6 (data not shown), the furfural yield and titer in MIBK 
alone was used to compare the effects of different parameters. Furfural titer and yield in MIBK was doubled when the temperature was increased from $150^{\circ} \mathrm{C}$ to $160^{\circ} \mathrm{C}$, and a further increase of yield and concentration was observed when temperature was raised to $170{ }^{\circ} \mathrm{C}$. This result was in consistent with the results obtained from other studies, which indicated that higher temperature was beneficial to furfural production (Zhang et al., 2017a; Zhang et al., 2013). Acid, as a proton donor, plays a crucial role in hydrolysis of hemicellulose into pentose and converting it into furfural. In the tested acid range $(0.4-0.6 \%)$, furfural production increased with acid concentration. Furfural titer and yield peaked at acid concentration of $0.6 \%$, reaching $6.32 \mathrm{~g} / \mathrm{l}$ and $38.62 \%$ respectively. To make the production cost-effective, it is also desirable to load as much lignocellulosic biomass as possible in a single batch. However, at high solid loading, mass and heat transfer could be negatively influenced, and reduced furfural production could be incurred. Increasing solid loading from 9.1 to $10.7 \%$ did not apparently influence the furfural production as a similar yield was achieved, but a higher furfural titer was obtained due to a higher loading. However, a drop of both furfural concentration and yield in MIBK was noticed when solid loading was increased to $12.3 \%$. This decrease could be partially attributed to the limited mass transfer as a very viscous slurry was formed during the reaction. To further improve the furfural production, reaction time ranging from 30 to 60 min was tested, and it was found that compared with $30 \mathrm{~min}$ reaction time, a reaction time of $60 \mathrm{~min}$ led to $15 \%$ higher yield in MIBK, and the furfural titer could reach $11.04 \mathrm{~g} / \mathrm{l}$ consequently.

To understand the lignin and cellulose fate under different conditions, the compositional distribution of pretreated biomass was examined (Table 6.1). Varying the conditions did not affect the lignin removal as about $60 \%$ removal was observed 
under all the tested conditions. In contrast, the xylan was more susceptible to conditions variation. Increasing temperature from $150{ }^{\circ} \mathrm{C}$ to $170{ }^{\circ} \mathrm{C}$ led to $7 \%$ more xylan removal at acid concentration of $0.4 \%$, and a near $12 \%$ more xylan removal was obtained when acid concentration was raised to $0.6 \%$. Under all the conditions tested, more than $90 \%$ glucan was preserved and recovered as a solid residue. Thus, aqueous $\mathrm{ChCl}$ selectively remove most xylan and a large fraction of lignin from switchgrass, while most glucan could be preserved. Taking furfural production, lignin removal, and cellulose preservation into consideration, the following conditions: $170{ }^{\circ} \mathrm{C}, 0.6 \%$ $\mathrm{H}_{2} \mathrm{SO}_{4}, 10.7 \%$ solid loading, and a reaction time of $60 \mathrm{~min}$, were selected as the optimum conditions for one-pot furfural production from switchgrass.

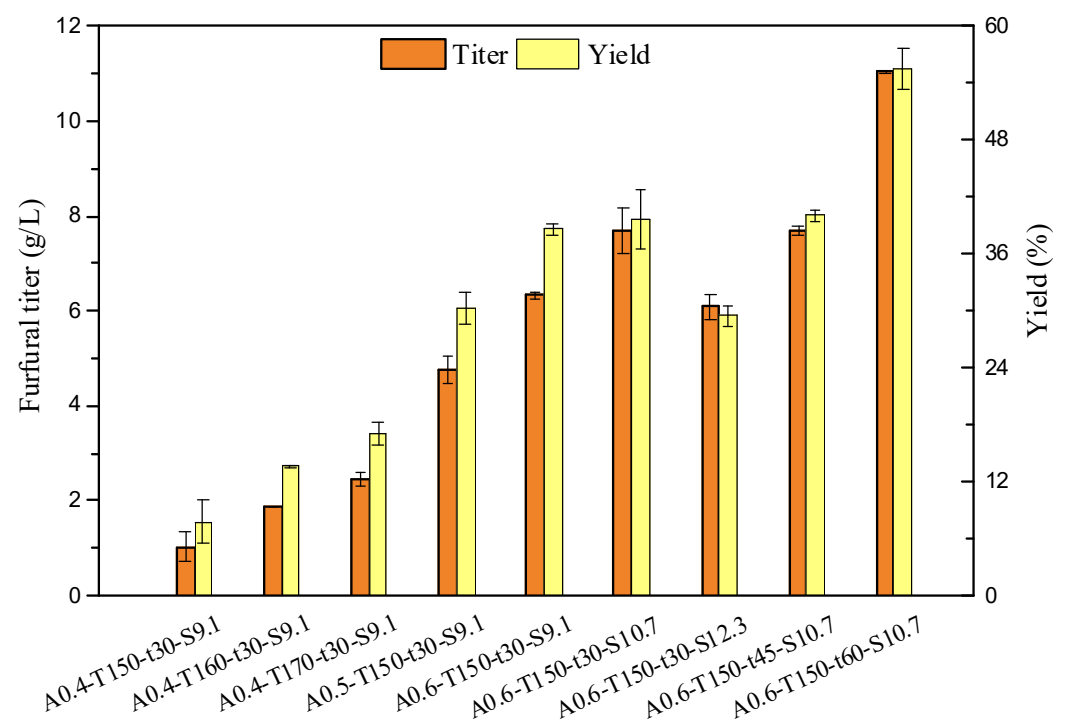

Figure 6.2 Furfural titer and yield in MIBK under different conditions 
Table 6.1 Component distribution and removal of switchgrass upon pretreatment under different conditions.

\begin{tabular}{|c|c|c|c|c|c|c|c|}
\hline \multirow[b]{2}{*}{ Conditions } & \multirow{2}{*}{$\begin{array}{l}\text { Solid } \\
\text { recovery } \\
(\%)\end{array}$} & \multicolumn{3}{|c|}{ Composition (\%) } & \multicolumn{3}{|c|}{ Removal (\%) } \\
\hline & & Glucan & Xylan & Lignin & Glucan & Xylan & Lignin \\
\hline A0.4-T150-t30-S9.1* & $51.00 \pm 0.57$ & $61.69 \pm 044$ & $10.61 \pm 0.10$ & $15.57 \pm 1.44$ & $9.01 \pm 3.56$ & $76.99 \pm 0.59$ & $61.29 \pm 5.74$ \\
\hline A0.4-T160-t30-S9.1 & $48.86 \pm 0.04$ & $65.02 \pm 1.20$ & $9.07 \pm 0.67$ & $14.39 \pm 0.56$ & $6.08 \pm 1.31$ & $81.15 \pm 1.37$ & $65.81 \pm 1.86$ \\
\hline A0.4-T170-t30-S9.1 & $48.47 \pm 0.73$ & $67.36 \pm 0.29$ & $7.82 \pm 0.31$ & $14.89 \pm 0.45$ & $3.47 \pm 1.15$ & $83.87 \pm 0.95$ & $64.87 \pm 2.27$ \\
\hline A $0.5-\mathrm{T} 170-\mathrm{t} 30-\mathrm{S} 9.1$ & $44.20 \pm 0.52$ & $71.92 \pm 0.04$ & $4.06 \pm 0.36$ & $15.38 \pm 0.09$ & $6.00 \pm 1.08$ & $92.36 \pm 0.80$ & $66.70 \pm 0.83$ \\
\hline A0.6-T170-t30-S9.1 & $43.24 \pm 0.22$ & $70.82 \pm 1.62$ & $2.30 \pm 0.44$ & $16.80 \pm 0.50$ & $9.46 \pm 1.00$ & $95.77 \pm 0.84$ & $64.44 \pm 1.24$ \\
\hline A0.6-T170-t30-S10.7 & $44.73 \pm 0.08$ & $70.68 \pm 1.40$ & $2.95 \pm 1.00$ & $17.54 \pm 0.03$ & $6.52 \pm 1.15$ & $94.38 \pm 1.91$ & $61.57 \pm 0.20$ \\
\hline A0.6-T170-t30-S12.3 & $53.92 \pm 0.07$ & $62.62 \pm 0.88$ & $5.85 \pm 0.21$ & $16.34 \pm 1.14$ & $0.16 \pm 0.85$ & $86.58 \pm 0.71$ & $56.89 \pm 4.34$ \\
\hline A0.6-T170-t45-S10.7 & $45.04 \pm 0.38$ & $72.91 \pm 1.10$ & $3.42 \pm 0.13$ & $17.84 \pm 0.89$ & $2.92 \pm 0.64$ & $93.45 \pm 0.43$ & $60.54 \pm 1.63$ \\
\hline A0.6-T170-t60-S10.7 & $43.04 \pm 0.03$ & $73.28 \pm 0.48$ & $2.34 \pm 0.26$ & $17.90 \pm 0.33$ & $6.74 \pm 0.69$ & $95.71 \pm 0.69$ & $62.15 \pm 0.66$ \\
\hline CTL & $58.81 \pm 0.24$ & $54.44 \pm 0.58$ & $8.40 \pm 0.03$ & $24.73 \pm 0.25$ & $7.20 \pm 0.98$ & $78.80 \pm 0.07$ & $28.78 \pm 0.61$ \\
\hline
\end{tabular}

*A: acid $\left(\mathrm{H}_{2} \mathrm{SO}_{4}\right)$ loading (wt\%); T: temperature $\left({ }^{\circ} \mathrm{C}\right)$; t: reaction time $(\mathrm{min})$; S: solid loading $(\%)$ 


\subsubsection{Comparison of performance between aqueous $\mathrm{ChCl} / \mathrm{MIBK}$ and $\mathrm{H}_{2} \mathrm{O} / \mathrm{MIBK}$}

Although decent furfural yield could be obtained from aqueous $\mathrm{ChCl} / \mathrm{MIBK}$ biphasic system, the question remains to be answered was whether it could really outperform a $\mathrm{H}_{2} \mathrm{O} / \mathrm{MIBK}$ biphasic system. Therefore, a comparison between aqueous $\mathrm{ChCl} / \mathrm{MIBK}$ and $\mathrm{H}_{2} \mathrm{O} / \mathrm{MIBK}$ was conducted under the optimized conditions from section 3.1. With aqueous $\mathrm{ChCl} / \mathrm{MIBK}$ biphasic system, an overall furfural yield of $84.04 \%$ was achieved, with a yield of $55.57 \%$ in MIBK phase and $28.47 \%$ in aqueous phase, corresponding to $11.04 \mathrm{~g} / \mathrm{L}$ and $5.66 \mathrm{~g} / \mathrm{L}$ furfural, respectively (Figure 6.2\&6.3). In contrast, with $\mathrm{H}_{2} \mathrm{O} / \mathrm{MIBK}$ biphasic system, only $0.45 \mathrm{~g} / \mathrm{L}$ furfural was obtained in MIBK phase while negligible furfural was detected in aqueous phase (Figure 6.2). As a result, the furfural yield from $\mathrm{H}_{2} \mathrm{O} / \mathrm{MIBK}$ was only $3 \%$ (Figure 6.2). Compared with $\mathrm{H}_{2} \mathrm{O} / \mathrm{MIBK}$, aqueous $\mathrm{ChCl} / \mathrm{MIBK}$ removed $17 \%$ more xylan and $34 \%$ more lignin. Glucan removal did not change much in both solvent systems, with more than $90 \%$ preserved. It appeared that aqueous $\mathrm{ChCl}$ worked as a better solvent for xylan and lignin dissolution, and accelerated the xylose dehydration to furfural. The better performance of aqueous $\mathrm{ChCl}$ could be attributed to its solvation effects. In water, when $\mathrm{H}_{2} \mathrm{SO}_{4}$ was at low concentration, due to the solvation of the acidic proton by $\mathrm{H}_{2} \mathrm{O}$ molecule, the insufficient protons donated by $\mathrm{H}_{2} \mathrm{SO}_{4}$ could not efficiently cleave those complex bonds in lignocellulosic biomass (eg. ether bonds, glycosidic bonds). On the contrary, the proton activity could be enhanced by aqueous $\mathrm{ChCl}$. Similar solvation effects by other organic solvent (eg. $\gamma$-valerolactone, dioxane, tetrahydrofuran) have also been demonstrated by a previous study (Mellmer et al., 2014). In addition, the presence of chloride could facilitate the isomerization of xylose to xylulose, and improved the selectivity of furfural production. Consequently, a 
higher furfural titer and yield could be obtained in aqueous $\mathrm{ChCl} / \mathrm{MIBK}$. In addition, enzymatic hydrolysis of cellulose pulps revealed that the one from aqueous $\mathrm{ChCl} / \mathrm{MIBK}$ gave a $100 \%$ digestibility, which was $30 \%$ higher than that from $\mathrm{H}_{2} \mathrm{O}$ /MIBK (Figure 6.3.). Such difference could be explained by a lower lignin content in cellulose pulp form aqueous $\mathrm{ChCl} / \mathrm{MIBK}$, which enhances the cellulase access to cellulose. Overall, the aqueous $\mathrm{ChCl} / \mathrm{MIBK}$ outperform $\mathrm{H}_{2} \mathrm{O} / \mathrm{MIBK}$ in terms of furfural production, lignin removal and cellulose digestibility.

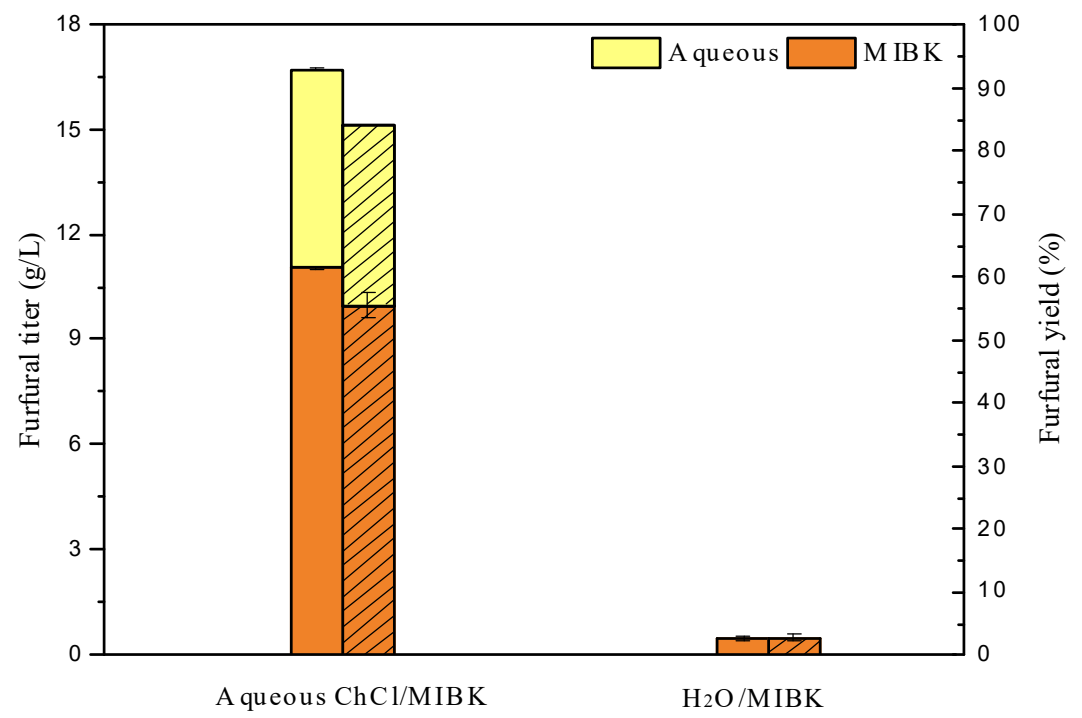

Figure 6.3 Furfural titer and yield from aqueous $\mathrm{ChCl} / \mathrm{MIBK}$ and $\mathrm{H}_{2} \mathrm{O} / \mathrm{MIBK}$ in onepot process. 


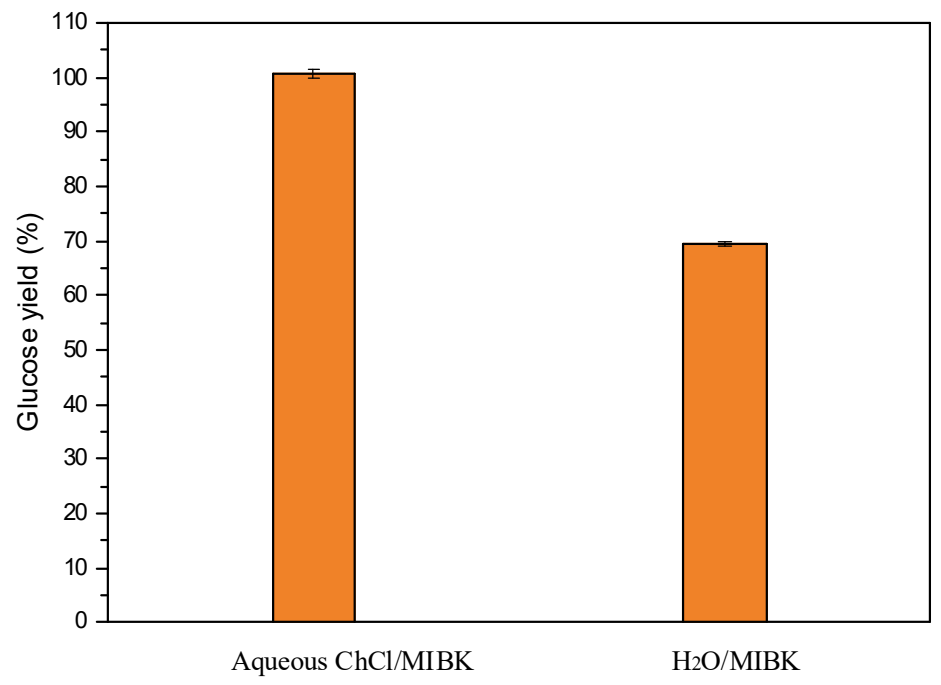

Figure 6.4 Digestibility of cellulose pulp recovered from one-pot process.

\subsubsection{Lignin characterization}

After one-pot process, two major lignin streams were formed. One was the lignin solubilized in aqueous $\mathrm{ChCl}$ (L-1), which can be recovered via precipitation from the liquor. Another was the lignin retained in the cellulose pulp, which can be recovered after enzymatic hydrolysis of cellulose (L-2). Lignin is a valuable resource that can be valorized into valuable chemicals, such as aromatics, lipid and polyhydroxyalkanoates, and advanced materials (Kai et al., 2016; Linger et al., 2014; Xie et al., 2017). However, the selection of valorization routes largely depends on lignin properties. Therefore, to fully understand the lignin chemical, thermal, and physical properties is of great importance.

2D HSQC NMR was used to understand the chemical transformation of these two lignin streams, and the main ${ }^{13} \mathrm{C}-{ }^{1} \mathrm{H}$ cross-signals in the HSQC spectra of switchgrass lignin were assigned according to previous studies (Figure 6.4) (Samuel et al., 2011; Samuel et al., 2010). The results indicated that lignin solubilized in aqueous $\mathrm{ChCl}$ (L- 
1) experienced a complete cleavage of the $\beta-\mathrm{O}-4$ bonds (A) (Figure 6.4).

Interestingly, a peak corresponding to Hibbert's ketone (HK) was clearly shown in the L-1, further corroborating the cleavage of $\beta-\mathrm{O}-4$ linkages. In addition, there was no apparent signal in the carbohydrate anomeric regions $\left(\delta_{\mathrm{C}} / \delta_{\mathrm{H}} 90-105 / 3.9-5.4\right)$ (Yuan et al., 2011), suggesting that L-1 had a high purity with no carbohydrate retained. Purity analysis further confirmed that this lignin had a purity as high as $93.2 \%$ with no carbohydrate detected. A lignin with high purity may find its application in many fields, such as antioxidant, resin synthesis, and polyurethane synthesis (Lauberts et al., 2017; Lora \& Glasser, 2002). The residual lignin after enzymatic hydrolysis of the cellulose pulp (L-2) showed a structure with more similarity to native lignin, with $\beta$ O-4 linkages as the major peak in the aliphatic regions (Figure 6.4). C-C bonds, such as $\beta-5(B)$ and $\beta-\beta(C)$, were also detected in L-2. Lignin with ether bonds well preserved is beneficial to aromatic monomers production via catalytic depolymerization route (Phongpreecha et al., 2017; Renders et al., 2017). However, due to the low dissociation energy of $\beta-\mathrm{O}-4$ linkages, it often experiences significant cleavage during pretreatment. Recently, the lignin-first pretreatment was proposed, aiming at upgrading lignin into aromatic monomers from untreated lignocellulosic biomass (Phongpreecha et al., 2017; Renders et al., 2017).The lignin-first methods hold great promise for lignin valorization by taking advantage of the preserved $\beta-\mathrm{O}-4$ linkages in untreated lignocellulosic biomass, but efficient recycling the catalyst from the mixture of polysaccharide and catalyst remains challenging (Sun et al., 2018).

Given the nearly complete removal of polysaccharide and well-preserved $\beta$-O-4 linkages, the residual lignin after enzymatic digestion has a great potential to be valorized into monomers while ensuring better catalyst recycling. 
Syringyl (S) units, guaiacyl (G) units, and $p$-coumarate ( $p$-CA) were dominant peaks across the aromatic regions in native lignin, L-1 and L-2. However, the peaks for G units in L-1 showed a faint signal compared with L-2 and native lignin (NL). This was possibly due to the re-localization of aromatic rings upon cleavage of $\beta-\mathrm{O}-4$ linkages in L-1 (Chen et al., 2017). An appreciable amount of ferulate (FA) could be detected in both native lignin and L-2, but was absent in L-1. In The ligninpolysaccharide cross-linking is formed via cross-coupling of ferulate with lignin monomers and oligomers (Kim \& Ralph, 2010). The absence of FA in L-1 suggested the cleavage of lignin-polysaccharide in one-pot process, which facilitated the fractionation of lignin and xylan from switchgrass. 

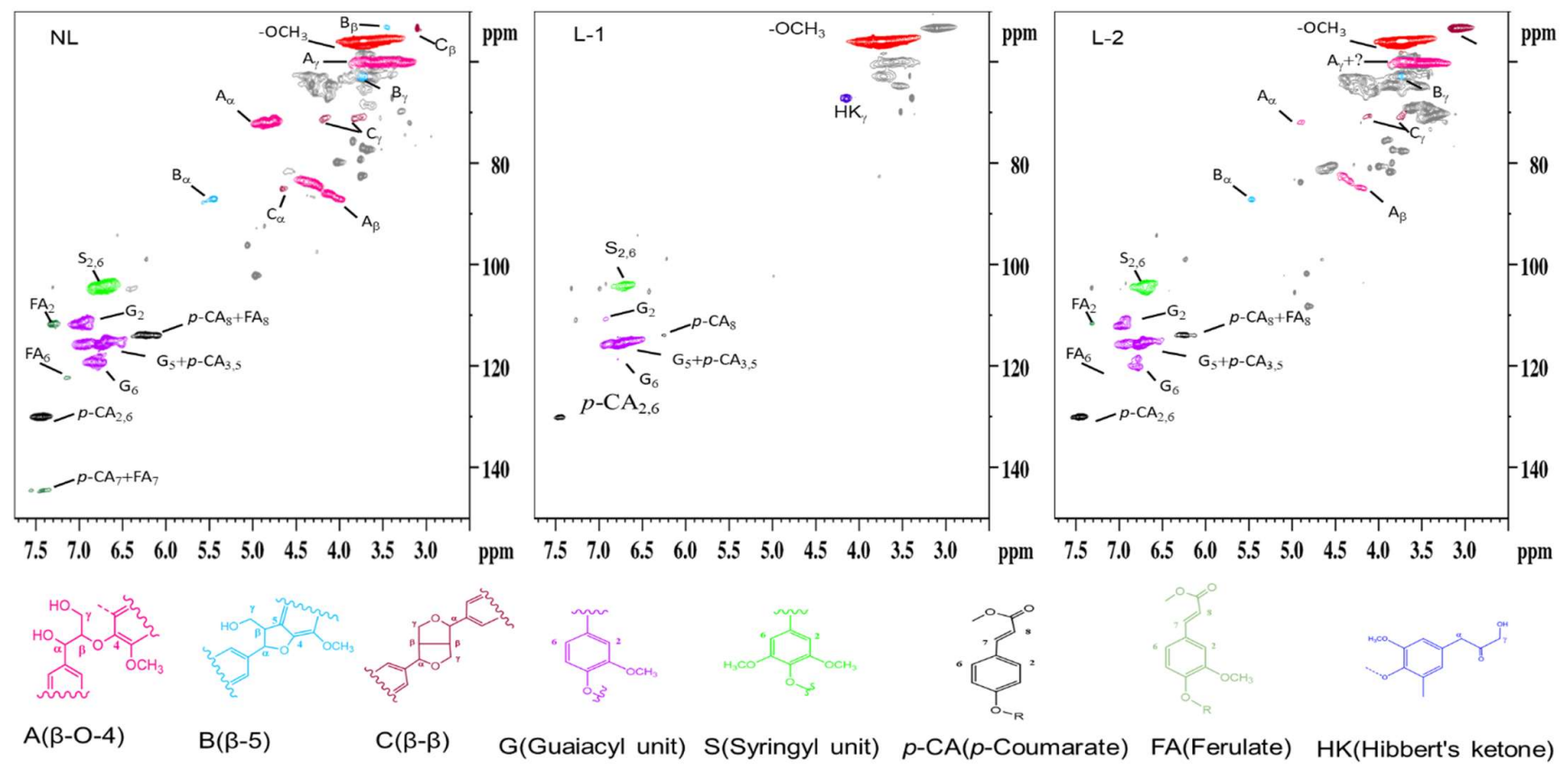

Figure 6.5 2D HSQC NMR spectra of lignin. L-1and L-2 represent lignin recovered from one-pot process and enzymatic hydrolysis. 


\subsubsection{Recyclability of aqueous $\mathrm{ChCl}$}

Recycling solvent could potentially lower the cost associated with waste stream management and chemicals consumption. Thus, the recyclability of aqueous $\mathrm{ChCl}$ was investigated. Furfural yield and titer in MIBK were similar for the first two cycles, reaching near 50\% and $11 \mathrm{~g} / \mathrm{L}$ (Figure 6.5). A slight decrease of furfural production was observed for the $3^{\text {rd }}$ and $4^{\text {th }}$ cycles. Despite this, furfural yield over $40 \%$ in MIBK could still be achieved during the $3^{\text {rd }}$ and $4^{\text {th }}$ cycles (Figure 6.5 ). Compositional analysis revealed that with increased cycles, the capability of xylan solubilization by aqueous $\mathrm{ChCl}$ remained unchanged (Figure 6.6), reaching 96\% through all cycles. However, a gradually increased lignin content in cellulose pulp and a decreased lignin removal was noticed (Figure 6.6\& 6.7). As shown in Figure 6.8, lignin yield for each cycle and cumulative lignin yield did not exceed $70 \%$, suggesting that a large fractionation of solubilized lignin was retained in the recycled aqueous $\mathrm{ChCl}$. This lignin could react with carbohydrate (xylose, glucose) and their derived compounds (eg. furfural) to form pseudo lignin, and lowered the furfural yield while increased the lignin content. Due to the increased lignin content, sugar yield was decreased from $100 \%$ in the $1^{\text {st }}$ cycle to $80 \%$ in the $2^{\text {nd }}$ cycle, and slightly decreased to $77 \%$ and $79 \%$ in the $3^{\text {rd }}$ and $4^{\text {th }}$ cycles (Figure 6.8 ). Future studies need to be focused on developing better strategies to recover lignin from the liquor, and remove other impurities to further improve the performance of the recycled aqueous $\mathrm{ChCl}$. 


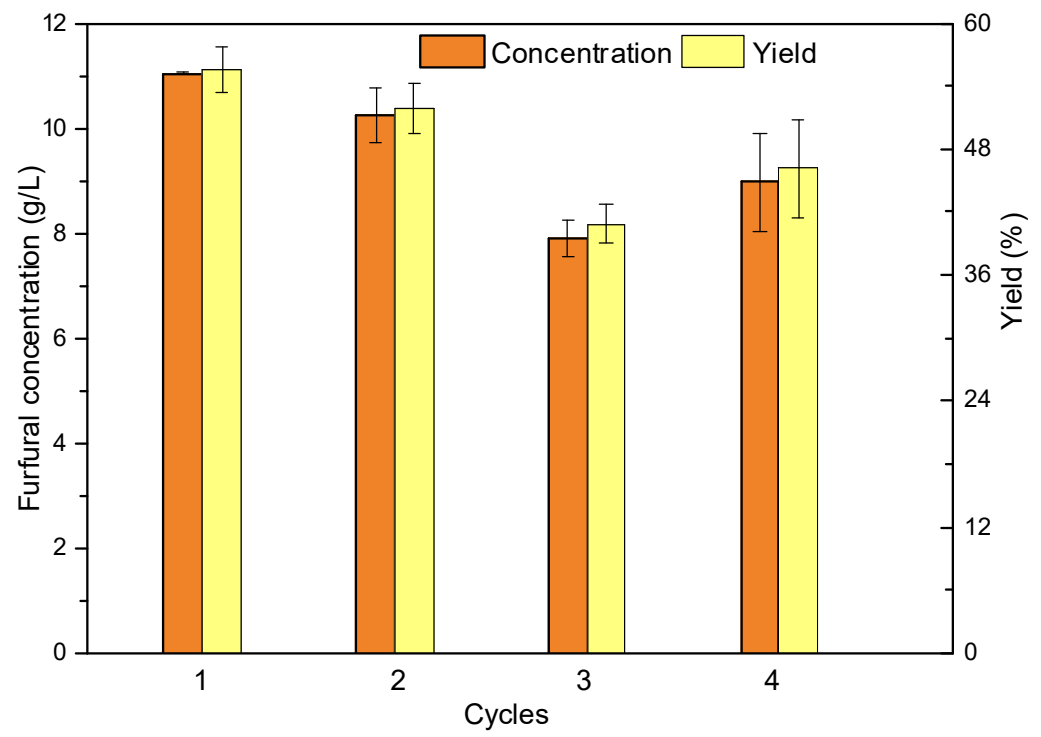

Figure 6.6 Furfural concentration and yield in MIBK during different cycles.

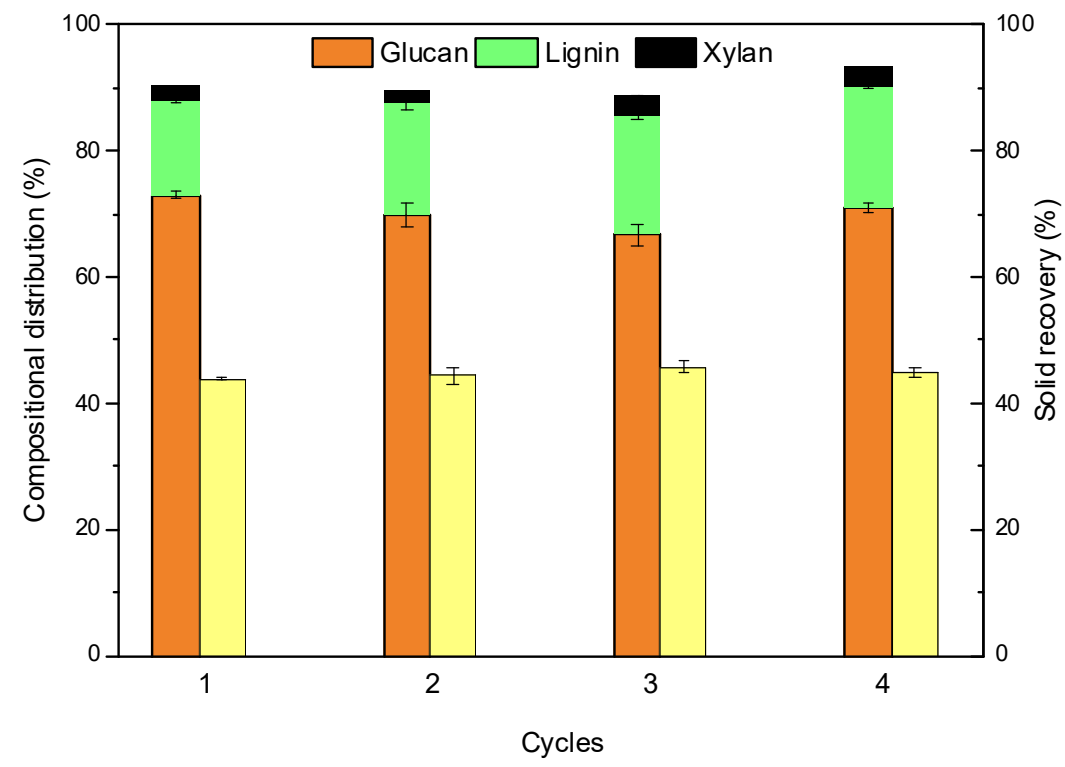

Figure 6.7 Compositional distribution of pretreated switchgrass during different cycles. 


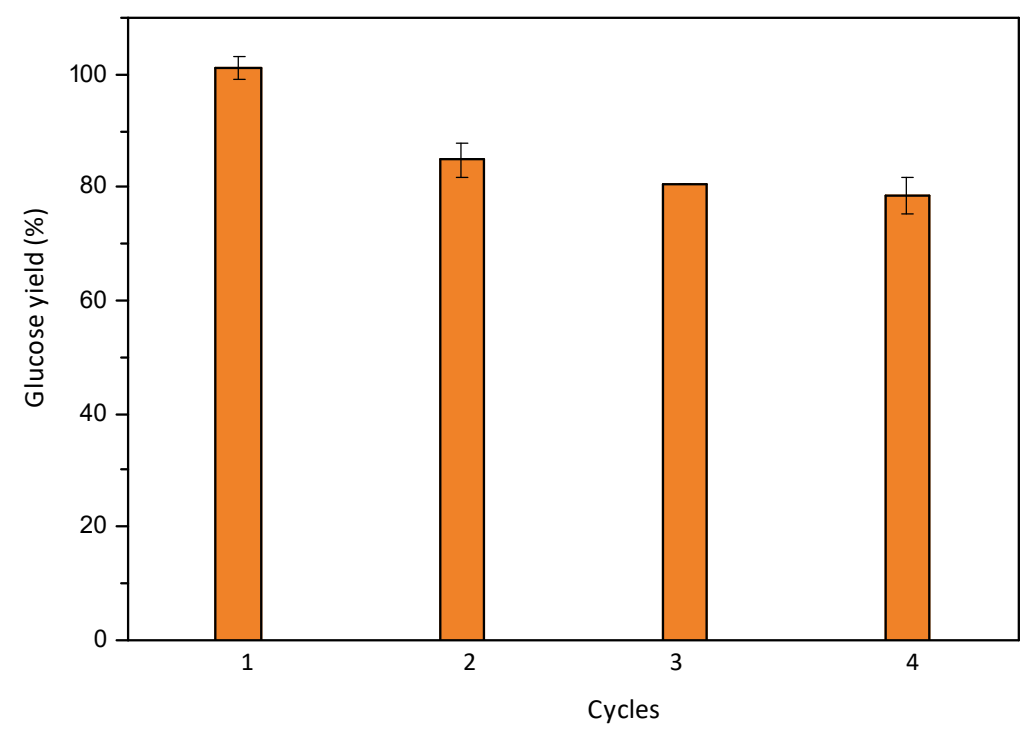

Figure 6.8 Digestibility of switchgrass from different cycles of one-pot treatment.

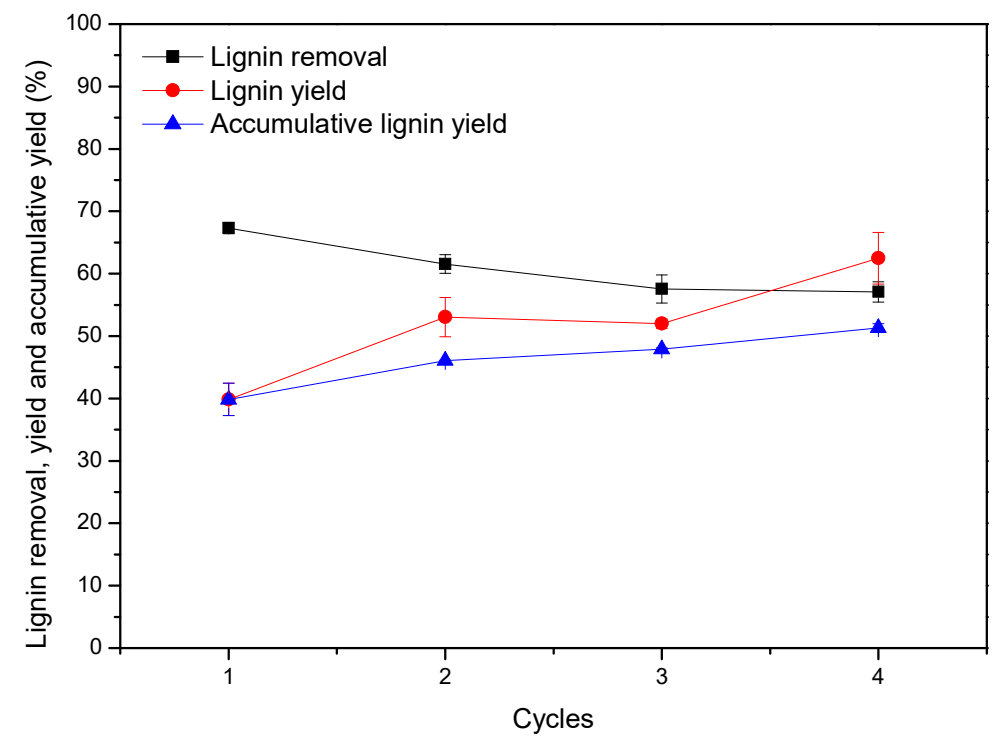

Figure 6.9 Lignin removal and yield in different cycles 


\subsection{Conclusion}

Aqueous $\mathrm{ChCl} / \mathrm{MIBK}$ biphasic system showed good performance in producing furfural from untreated switchgrass, with a maximum yield of $84.0 \%$ achieved under optimized conditions. In addition to furfural production, the cellulose pulp with $93.2 \%$ of its initial cellulose retained showed a $100 \%$ digestibility. The solvent system also solubilized $62 \%$ lignin, which was recovered with a high purity (93.2\%). The lignin retained in cellulose pulp can be recovered after enzymatic digestion, and showed a similar structure with native lignin, with the $\beta-\mathrm{O}-4$ linkages preserved. Overall, this study developed a novel aqueous $\mathrm{ChCl} / \mathrm{MIBK}$ solvent system with great potential to valorize all the major components from untreated lignocellulosic biomass into valueadded products in via a one-pot process. 


\section{Chapter 7 Conclusions and suggested future work}

\subsection{Conclusions}

This work investigated the biomass conversion into renewable chemicals and valorizable lignin by using quaternary ammonium salts-based green solvents. Based on the results obtained in this work, the following conclusions can be drawn:

- Pretreatment using quaternary ammonium salts-based solvents were effective for selectively removing lignin and xylan, while preserving most of cellulose. The solubilized lignin can be easily recovered, and demonstrated properties beneficial to its upgrading. By systematically controlling the pretreatment conditions and solvent compositions, lignin properties can be tailored while delignification was not compromised.

- Quaternary ammonium salt-based solvents were very effective for lignocellulosic biomass fractionation at high solid loading. The resultant cellulose pulp can be efficiently enzymatically hydrolyzed into glucose under high solid loading and low enzyme loading. The thus obtained highly concentrated hydrolysate is a suitable substrate for producing biofuels and chemicals at high titer via biological conversion.

- Quaternary ammonium salt-based solvents can effectively solubilize xylan. Recycling the solvents led to enrichment of xylan-derived sugars in the pretreatment liquor. The xylose-rich pretreatment liquor can be directly upgraded into furfural with high yield.

- One-pot process using biphasic system consisted of recyclable aqueous choline chloride and MIBK can convert lignocellulosic biomass into furfural, high 
purity lignin and digestible cellulose pulp in one step. The recovered lignin had a high purity with no carbohydrate remaining, and the cellulose pulp gave a glucose yield of $100 \%$ upon enzymatic hydrolysis. This one-pot process has potential to be used for clean fractionation of lignocellulosic biomass, and maximize the utilization of the major components.

\subsection{Suggested future work}

\subsubsection{Understand the mechanism for $\beta$-O-4 linkage stabilization by choline chloride}

The results from Chapters 2 and 5 revealed that the presence of choline chloride at a higher concentration can stabilize $\beta-\mathrm{O}-4$ linkage in lignin during pretreatment. However, the exact mechanism remains unclear. Understanding the mechanism will help design better pretreatment solvents that can actively stabilize the $\beta-O-4$ linkages in lignin during pretreatment. By using computational methods, such as density functional calculations, the mechanisms are expected to be better understood.

\subsubsection{Lignin upgrading into value-added products}

This work present various processes to extract lignin with different unique properties beneficial to upgrading. The lignin with abundant $\beta-O-4$ linkages can be used for aromatic monomers production upon catalytic depolymerization. The lignin with good thermal stability and purity may find its application in carbon fiber production. Future work can explore the valorization potential of such lignin, and understand how lignin properties affect products' properties. It is expected that by valorizing such lignin into value-added products, the revenue from lignocellulosic biomass can be further increased. 


\subsubsection{Cellulose-based advanced materials production}

The results from this work indicated that cellulose pulp with small amount of lignin and hemicellulose can be produced using different solvents. In addition to converting cellulose into chemicals as demonstrated in this work, the cellulose pulp can also be used for manufacturing advanced materials, such as cellulose nanofiber, cellulose nanocrystal, nanocellululose-based aerogels, which can find their applications in energy storage, drug delivery, and many other fields. Manufacturing cellulose pulp into advanced materials can expand the spectrum of products from lignocellulosic biomass, increase the revenue for biorefinery, and make biorefinery versatile.

\subsubsection{One-pot production of jet-fuel precursors from lignocellulosic biomass}

Furans can react with acetone to form long chain adducts, which can be upgraded into valuable jet fuels after hydrodeoxygenation. The results from Chapter 2 suggested that aqueous choline chloride-based solvents can form biphasic system with acetone, and catalyze the hemicellulose conversion into furfural with high yield. By further designing the solvent into a bifunctional system which can catalyze both polysaccharide conversion into furans, and aldol condensation between furans and acetone, jet fuel precursors can be facilely produced from lignocellulosic biomass via a one-pot process. It is expected that this novel one-pot system will simplify the processes for producing jet fuels, and reduce chemical consumption and waste generation. 


\section{References}

Abbott, A.P., Boothby, D., Capper, G., Davies, D.L., Rasheed, R.K. 2004. Deep eutectic solvents formed between choline chloride and carboxylic acids: versatile alternatives to ionic liquids. J. Am. Chem. Soc., 126(29), 9142-9147.

Adlakha, N., Yazdani, S.S. 2015. Efficient production of (R, R)-2, 3-butanediol from cellulosic hydrolysate using Paenibacilluspolymyxa ICGEB2008. J. Ind. Microbiol. Biotechnol., 42(1), 21-28.

Alonso, D.M., Hakim, S.H., Zhou, S., Won, W., Hosseinaei, O., Tao, J., GarciaNegron, V., Motagamwala, A.H., Mellmer, M.A., Huang, K. 2017. Increasing the revenue from lignocellulosic biomass: Maximizing feedstock utilization. Sci. Adv., 3(5), e1603301.

Alonso, D.M., Wettstein, S.G., Dumesic, J.A. 2013. Gamma-valerolactone, a sustainable platform molecule derived from lignocellulosic biomass. Green Chem., 15(3), 584-595.

Alvarez-Vasco, C., Ma, R., Quintero, M., Guo, M., Geleynse, S., Ramasamy, K.K., Wolcott, M., Zhang, X. 2016. Unique low-molecular-weight lignin with high purity extracted from wood by deep eutectic solvents (DES): a source of lignin for valorization. Green Chem., 18(19), 5133-5141.

An, Y.X., Zong, M.H., Wu, H., Li, N. 2015. Pretreatment of lignocellulosic biomass with renewable cholinium ionic liquids: Biomass fractionation, enzymatic digestion and ionic liquid reuse. Bioresour Technol, 192, 165-71.

Antonyraj, C.A., Kim, B., Kim, Y., Shin, S., Lee, K.-Y., Kim, I., Cho, J.K. 2014. Heterogeneous selective oxidation of 5-hydroxymethyl-2-furfural (HMF) into 2,5-diformylfuran catalyzed by vanadium supported activated carbon in MIBK, extracting solvent for HMF. Catal. Commun., 57, 64-68.

Araji, N., Madjinza, D.D., Chatel, G., Moores, A., Jerome, F., De Oliveira Vigier, K. 2017. Synthesis of maleic and fumaric acids from furfural in the presence of betaine hydrochloride and hydrogen peroxide. Green Chem., 19(1), 98-101.

Bayu, A., Guan, G., Karnjanakom, S., Hao, X., Kusakabe, K., Abudula, A. 2016. Catalytic synthesis of levulinic acid and formic acid from glucose in choline chloride aqueous solution. ChemistrySelect, 1(2), 180-188.

Bekiaris, G., Lindedam, J., Peltre, C., Decker, S.R., Turner, G.B., Magid, J., Bruun, S. 2015. Rapid estimation of sugar release from winter wheat straw during bioethanol production using FTIR-photoacoustic spectroscopy. Biotechnol. Biofuels, 8(1), 85.

Bian, H., Chen, L., Dai, H., Zhu, J.Y. 2017. Integrated production of lignin containing cellulose nanocrystals (LCNC) and nanofibrils (LCNF) using an easily recyclable di-carboxylic acid. Carbohydr Polym, 167, 167-176.

Binod, P., Satyanagalakshmi, K., Sindhu, R., Janu, K.U., Sukumaran, R.K., Pandey, A. 2012. Short duration microwave assisted pretreatment enhances the enzymatic saccharification and fermentable sugar yield from sugarcane bagasse. Renewable Energy, 37(1), 109-116.

Börjesson, J., Engqvist, M., Sipos, B., Tjerneld, F. 2007. Effect of poly(ethylene glycol) on enzymatic hydrolysis and adsorption of cellulase enzymes to pretreated lignocellulose. Enzyme Microb. Technol., 41(1), 186-195. 
Brandt, A., Chen, L., van Dongen, B.E., Welton, T., Hallett, J.P. 2015. Structural changes in lignins isolated using an acidic ionic liquid water mixture. Green Chem., 17(11), 5019-5034.

Brandt-Talbot, A., Gschwend, F.J.V., Fennell, P.S., Lammens, T.M., Tan, B., Weale, J., Hallett, J.P. 2017. An economically viable ionic liquid for the fractionation of lignocellulosic biomass. Green Chem., 19(13), 3078-3102.

Brodeur, G., Yau, E., Badal, K., Collier, J., Ramachandran, K., Ramakrishnan, S. 2011. Chemical and physicochemical pretreatment of lignocellulosic biomass: a review. Enzyme Res, 2011, 787532.

Bubalo, M.C., Tušek, A.J., Vinković, M., Radošević, K., Srček, V.G., Redovniković, I.R. 2015. Cholinium-based deep eutectic solvents and ionic liquids for lipasecatalyzed synthesis of butyl acetate. J. Mol. Catal. B: Enzym., 122, 188-198.

Cai, C.M., Zhang, T., Kumar, R., Wyman, C.E. 2014. Integrated furfural production as a renewable fuel and chemical platform from lignocellulosic biomass. $J$. Chem. Technol. Biotechnol., 89(1), 2-10.

Chen, C.-Z., Li, M.-F., Wu, Y.-Y., Sun, R.-C. 2014. Integration of ambient formic acid process and alkaline hydrogen peroxide post-treatment of furfural residue to enhance enzymatic hydrolysis. Ind. Eng. Chem. Res., 53(33), 12935-12942.

Chen, L., Dou, J., Ma, Q., Li, N., Wu, R., Bian, H., Yelle, D.J., Vuorinen, T., Fu, S., Pan, X. 2017. Rapid and near-complete dissolution of wood lignin at $\leq 80^{\circ} \mathrm{C}$ by a recyclable acid hydrotrope. Sci. Adv., 3(9), e1701735.

Chen, X., Kuhn, E., Jennings, E.W., Nelson, R., Tao, L., Zhang, M., Tucker, M.P. 2016. DMR (deacetylation and mechanical refining) processing of corn stover achieves high monomeric sugar concentrations $(230 \mathrm{~g} \mathrm{~L}-1)$ during enzymatic hydrolysis and high ethanol concentrations $(>10 \% \mathrm{v} / \mathrm{v})$ during fermentation without hydrolysate purification or concentration. Energy Environ. Sci., 9(4), $1237-1245$.

Chen, Z., Bai, X., A, L., Wan, C. 2018a. High-Solid Lignocellulose Processing Enabled by Natural Deep Eutectic Solvent for Lignin Extraction and Industrially Relevant Production of Renewable Chemicals. ACS Sustainable Chem. Eng.

Chen, Z., Reznicek, W.D., Wan, C. 2018b. Aqueous Choline Chloride: A Novel Solvent for Switchgrass Fractionation and Subsequent Hemicellulose Conversion into Furfural. ACS Sustainable Chem. Eng., 6(5), 6910-6919.

Chen, Z., Wan, C. 2018. Ultrafast fractionation of lignocellulosic biomass by microwave-assisted deep eutectic solvent pretreatment. Bioresour. Technol., 250, $532-537$.

Cui, Q., Peng, X., Yao, X.-H., Wei, Z.-F., Luo, M., Wang, W., Zhao, C.-J., Fu, Y.-J., Zu, Y.-G. 2015. Deep eutectic solvent-based microwave-assisted extraction of genistin, genistein and apigenin from pigeon pea roots. Sep. Purif. Technol., 150, 63-72.

da Silva, F.L., de Oliveira Campos, A., dos Santos, D.A., de Oliveira Júnior, S.D., de Araújo Padilha, C.E., de Sousa Junior, F.C., de Macedo, G.R., dos Santos, E.S. 2018. Pretreatments of Carnauba (Copernicia prunifera) straw residue for production of cellulolytic enzymes by Trichorderma reesei CCT-2768 by solid state fermentation. Renewable Energy, 116, 299-308. 
Dadi, A.P., Varanasi, S., Schall, C.A. 2006. Enhancement of cellulose saccharification kinetics using an ionic liquid pretreatment step. Biotechnol Bioeng, 95(5), 904-910.

Dai, Y., Zhang, H.-S., Huan, B., He, Y. 2017. Enhancing the enzymatic saccharification of bamboo shoot shell by sequential biological pretreatment with Galactomyces sp. CCZU11-1 and deep eutectic solvent extraction. Bioprocess. Biosyst. Eng., 40(9), 1427-1436.

Di Marino, D., Aniko, V., Stocco, A., Kriescher, S., Wessling, M. 2017. Emulsion electro-oxidation of kraft lignin. Green Chem., 19(20), 4778-4784.

Di Marino, D., Stöckmann, D., Kriescher, S., Stiefel, S., Wessling, M.J.G.C. 2016. Electrochemical depolymerisation of lignin in a deep eutectic solvent. Green Chem., 18(22), 6021-6028.

Dien, B.S., Sarath, G., Pedersen, J.F., Sattler, S.E., Chen, H., Funnell-Harris, D.L., Nichols, N.N., Cotta, M.A. 2009. Improved Sugar Conversion and Ethanol Yield for Forage Sorghum (Sorghum bicolor L. Moench) Lines with Reduced Lignin Contents. Bioenergy Res., 2(3), 153-164.

Durand, E., Lecomte, J., Baréa, B., Dubreucq, E., Lortie, R., Villeneuve, P.J.G.C. 2013. Evaluation of deep eutectic solvent-water binary mixtures for lipasecatalyzed lipophilization of phenolic acids. Green Chem., 15(8), 2275-2282.

Dussan, K., Girisuta, B., Lopes, M., Leahy, J.J., Hayes, M.H. 2016. Effects of Soluble Lignin on the Formic Acid-Catalyzed Formation of Furfural: A Case Study for the Upgrading of Hemicellulose. ChemSusChem, 9(5), $492-504$.

Dutta, T., Papa, G., Wang, E., Sun, J., Isern, N.G., Cort, J.R., Simmons, B.A., Singh, S.J.A.S.C., Engineering. 2018. Characterization of Lignin Streams during Bionic Liquid-Based Pretreatment from Grass, Hardwood, and Softwood. ACS Sustainable Chem. Eng., 6(3), 3079-3090.

Enslow, K.R., Bell, A.T.J.C. 2015. The role of metal halides in enhancing the dehydration of xylose to furfural. Chemcatchem, 7(3), 479-489.

Faba, L., Díaz, E., Ordóñez, S. 2012. Aqueous-phase furfural-acetone aldol condensation over basic mixed oxides. Appl. Catal., B, 113-114, 201-211.

Faba, L., Díaz, E., Vega, A., Ordóñez, S. 2016. Hydrodeoxygenation of furfuralacetone condensation adducts to tridecane over platinum catalysts. Catal. Today, 269, 132-139.

Gao, C., Ren, J., Zhao, C., Kong, W., Dai, Q., Chen, Q., Liu, C., Sun, R. 2016. Xylanbased temperature/pH sensitive hydrogels for drug controlled release. Carbohydr Polym, 151, 189-197.

Geng, W., Jin, Y., Jameel, H., Park, S. 2015. Strategies to achieve high-solids enzymatic hydrolysis of dilute-acid pretreated corn stover. Bioresour. Technol., 187(Supplement C), 43-48.

Gong, G., Liu, D., Huang, Y. 2010. Microwave-assisted organic acid pretreatment for enzymatic hydrolysis of rice straw. Biosyst. Eng., 107(2), 67-73.

Gorke, J.T., Srienc, F., Kazlauskas, R.J. 2008. Hydrolase-catalyzed biotransformations in deep eutectic solvents. Chem Commun(10), 1235-7.

Gschwend, F.J., Malaret, F., Shinde, S., Brandt-Talbot, A., Hallett, J.P.J.G.C. 2018. Rapid pretreatment of Miscanthus using the low-cost ionic liquid triethylammonium hydrogen sulfate at elevated temperatures. Green Chem., 20(15), 3486-3498. 
Gunny, A.A.N., Arbain, D., Nashef, E.M., Jamal, P. 2015. Applicability evaluation of Deep Eutectic Solvents-Cellulase system for lignocellulose hydrolysis. Bioresour. Technol., 181, 297-302.

Guo, W., Hou, Y., Ren, S., Tian, S., Wu, W. 2013. Formation of deep eutectic solvents by phenols and choline chloride and their physical properties. $J$. Chem. Eng. Data, 58(4), 866-872.

Haghighi Mood, S., Hossein Golfeshan, A., Tabatabaei, M., Salehi Jouzani, G., Najafi, G.H., Gholami, M., Ardjmand, M. 2013. Lignocellulosic biomass to bioethanol, a comprehensive review with a focus on pretreatment. Renewable Sustainable Energy Rev., 27, 77-93.

Harish, B.S., Uppuluri, K.B., Anbazhagan, V. 2015. Synthesis of fibrinolytic active silver nanoparticle using wheat bran xylan as a reducing and stabilizing agent. Carbohydr Polym, 132, 104-10.

Hayyan, M., Hashim, M.A., Hayyan, A., Al-Saadi, M.A., AlNashef, I.M., Mirghani, M.E.S., Saheed, O.K. 2013. Are deep eutectic solvents benign or toxic? Chemosphere, 90(7), 2193-2195.

He, Y., Li, X., Ben, H., Xue, X., Yang, B. 2017. Lipid production from dilute alkali corn stover lignin by Rhodococcus strains. ACS Sustainable Chem. Eng., 5(3), 2302-2311.

Ho, H.C., Nguyen, N.A., Meek, K.M., Alonso, D.M., Hakim, S.H., Naskar, A.K. 2018. A Solvent-Free Synthesis of Lignin-Derived Renewable Carbon with Tunable Porosity for Supercapacitor Electrodes. ChemSusChem, 11(17), 29532959.

Hosseinaei, O., Harper, D.P., Bozell, J.J., Rials, T.G. 2016. Role of Physicochemical Structure of Organosolv Hardwood and Herbaceous Lignins on Carbon Fiber Performance. ACS Sustainable Chem. Eng., 4(10), 5785-5798.

Hou, X.-D., Li, A.-L., Lin, K.-P., Wang, Y.-Y., Kuang, Z.-Y., Cao, S.-L. 2018. Insight into the structure-function relationships of deep eutectic solvents during rice straw pretreatment. Bioresour. Technol., 249(Supplement C), 261267.

Hou, X.D., Feng, G.J., Ye, M., Huang, C.M., Zhang, Y. 2017. Significantly enhanced enzymatic hydrolysis of rice straw via a high-performance two-stage deep eutectic solvents synergistic pretreatment. Bioresour. Technol., 238, 139-146.

Hou, X.D., Xu, J., Li, N., Zong, M.H. 2015. Effect of anion structures on cholinium ionic liquids pretreatment of rice straw and the subsequent enzymatic hydrolysis. Biotechnol Bioeng, 112(1), 65-73.

Hu, Z., Wen, Z. 2008. Enhancing enzymatic digestibility of switchgrass by microwave-assisted alkali pretreatment. Biochem. Eng. J., 38(3), 369-378.

Huang, Y., Duan, Y., Qiu, S., Wang, M., Ju, C., Cao, H., Fang, Y., Tan, T. 2018. Lignin-first biorefinery: a reusable catalyst for lignin depolymerization and application of lignin oil to jet fuel aromatics and polyurethane feedstock. Sustainable Energy Fuels, 2(3), 637-647.

Jasiukaityte, E., Kunaver, M., Crestini, C. 2010. Lignin behaviour during wood liquefaction - Characterization by quantitative 31P, 13C NMR and sizeexclusion chromatography. Catal. Today, 156(1), 23-30.

Ji, X.-J., Huang, H., Ouyang, P.-K. 2011. Microbial 2, 3-butanediol production: a state-of-the-art review. Biotechnol. Adv., 29(3), 351-364. 
Kai, D., Tan, M.J., Chee, P.L., Chua, Y.K., Yap, Y.L., Loh, X.J. 2016. Towards lignin-based functional materials in a sustainable world. Green Chem., 18(5), 1175-1200.

Karp, E.M., Resch, M.G., Donohoe, B.S., Ciesielski, P.N., O’Brien, M.H., Nill, J.E., Mittal, A., Biddy, M.J., Beckham, G.T. 2015. Alkaline pretreatment of switchgrass. ACS Sustainable Chem. Eng., 3(7), 1479-1491.

Kim, H., Ralph, J. 2010. Solution-state 2D NMR of ball-milled plant cell wall gels in DMSO-d6/pyridine-d5. Org. Biomol. Chem., 8(3), 576-591.

Kim, K.H., Dutta, T., Sun, J., Simmons, B., Singh, S. 2018. Biomass pretreatment using deep eutectic solvents from lignin derived phenols. Green Chem., 20(4), 809-815.

Kristensen, J.B., Felby, C., Jorgensen, H. 2009. Yield-determining factors in highsolids enzymatic hydrolysis of lignocellulose. Biotechnol Biofuels, 2(1), 11.

Kucherov, F.A., Romashov, L.V., Galkin, K.I., Ananikov, V.P. 2018. Chemical Transformations of Biomass-Derived C6-Furanic Platform Chemicals for Sustainable Energy Research, Materials Science, and Synthetic Building Blocks. ACS Sustainable Chem. Eng.

Kumar, A.K., Parikh, B.S., Pravakar, M. 2016a. Natural deep eutectic solvent mediated pretreatment of rice straw: bioanalytical characterization of lignin extract and enzymatic hydrolysis of pretreated biomass residue. Environ. Sci. Pollut. Res., 23(10), 9265-9275.

Kumar, A.K., Parikh, B.S., Shah, E., Liu, L.Z., Cotta, M.A. 2016b. Cellulosic ethanol production from green solvent-pretreated rice straw. Biocatal. Agric. Biotechnol., 7(Supplement C), 14-23.

Kumar, P., Barrett, D.M., Delwiche, M.J., Stroeve, P. 2009. Methods for Pretreatment of Lignocellulosic Biomass for Efficient Hydrolysis and Biofuel Production. Ind. Eng. Chem. Res., 48(8), 3713-3729.

Lauberts, M., Sevastyanova, O., Ponomarenko, J., Dizhbite, T., Dobele, G., Volperts, A., Lauberte, L., Telysheva, G. 2017. Fractionation of technical lignin with ionic liquids as a method for improving purity and antioxidant activity. Ind. Crops Prod., 95, 512-520.

Li, C., Knierim, B., Manisseri, C., Arora, R., Scheller, H.V., Auer, M., Vogel, K.P., Simmons, B.A., Singh, S. 2010. Comparison of dilute acid and ionic liquid pretreatment of switchgrass: biomass recalcitrance, delignification and enzymatic saccharification. Bioresour. Technol., 101(13), 4900-4906.

Li, X., Jia, P., Wang, T. 2016. Furfural: a promising platform compound for sustainable production of C4 and C5 chemicals. ACS catalysis, 6(11), 76217640 .

Liang, X., Fu, Y., Chang, J. 2019. Effective separation, recovery and recycling of deep eutectic solvent after biomass fractionation with membrane-based methodology. Sep. Purif. Technol., 210, 409-416.

Ling, H., Cheng, K., Ge, J., Ping, W. 2017. Corncob Mild Alkaline Pretreatment for High 2,3-Butanediol Production by Spent Liquor Recycle Process. Bioenergy Res., 10(2), 566-574.

Linger, J.G., Vardon, D.R., Guarnieri, M.T., Karp, E.M., Hunsinger, G.B., Franden, M.A., Johnson, C.W., Chupka, G., Strathmann, T.J., Pienkos, P.T. 2014. Lignin valorization through integrated biological funneling and chemical catalysis. Proc. Natl. Acad. Sci. U.S.A, 111(33), 12013-12018. 
Liu, E.S., Li, M., Das, L., Pu, Y.Q., Frazier, T., Zhao, B.Y., Crocker, M., Ragauskas, A.J., Shi, J. 2018. Understanding Lignin Fractionation and Characterization from Engineered Switchgrass Treated by an Aqueous Ionic Liquid. ACS Sustainable Chem. Eng., 6(5), 6612-6623.

Liu, T., Williams, D.L., Pattathil, S., Li, M., Hahn, M.G., Hodge, D.B. 2014. Coupling alkaline pre-extraction with alkaline-oxidative post-treatment of corn stover to enhance enzymatic hydrolysis and fermentability. Biotechnol Biofuels, 7(1), 48.

Liu, Y., Chen, W., Xia, Q., Guo, B., Wang, Q., Liu, S., Liu, Y., Li, J., Yu, H. 2017. Efficient Cleavage of Lignin-Carbohydrate Complexes and Ultrafast Extraction of Lignin Oligomers from Wood Biomass by Microwave-Assisted Treatment with Deep Eutectic Solvent. ChemSusChem, 10(8), 1692-1700.

Loow, Y.-L., New, E.K., Yang, G.H., Ang, L.Y., Foo, L.Y.W., Wu, T.Y. 2017. Potential use of deep eutectic solvents to facilitate lignocellulosic biomass utilization and conversion. Cellulose, 24(9), 3591-3618.

Loow, Y.-L., Wu, T.Y., Yang, G.H., Ang, L.Y., New, E.K., Siow, L.F., Md. Jahim, J., Mohammad, A.W., Teoh, W.H. 2018. Deep eutectic solvent and inorganic salt pretreatment of lignocellulosic biomass for improving xylose recovery. Bioresour. Technol., 249, 818-825.

Lopes, M., Dussan, K., Leahy, J.J. 2017. Enhancing the conversion of D-xylose into furfural at low temperatures using chloride salts as co-catalysts: Catalytic combination of AlCl3 and formic acid. Chem. Eng. J., 323, 278-286.

Lora, J.H., Glasser, W.G. 2002. Recent Industrial Applications of Lignin: A Sustainable Alternative to Nonrenewable Materials. J. Polym. Environ., 10(1), $39-48$.

Luo, H., Abu-Omar, M.M. 2018. Lignin extraction and catalytic upgrading from genetically modified poplar. Green Chem., 20(3), 745-753.

Lupoi, J.S., Singh, S., Simmons, B.A., Henry, R.J. 2014. Assessment of Lignocellulosic Biomass Using Analytical Spectroscopy: an Evolution to High-Throughput Techniques. Bioenergy Res., 7(1), 1-23.

Luterbacher, J.S., Azarpira, A., Motagamwala, A.H., Lu, F., Ralph, J., Dumesic, J.A. 2015. Lignin monomer production integrated into the $\gamma$-valerolactone sugar platform. Energy Environ. Sci., 8(9), 2657-2663.

Lynam, J.G., Kumar, N., Wong, M.J. 2017. Deep eutectic solvents' ability to solubilize lignin, cellulose, and hemicellulose; thermal stability; and density. Bioresour Technol, 238, 684-689.

Mamman, A.S., Lee, J.M., Kim, Y.C., Hwang, I.T., Park, N.J., Hwang, Y.K., Chang, J.S., Hwang, J.S. 2008. Furfural: Hemicellulose/xylosederived biochemical. Biofuels, Bioprod. Biorefin., 2(5), 438-454.

Marcotullio, G., De Jong, W. 2010. Chloride ions enhance furfural formation from dxylose in dilute aqueous acidic solutions. Green Chem., 12(10), 1739-1746.

Mariscal, R., Maireles-Torres, P., Ojeda, M., Sádaba, I., Granados, M.L. 2016. Furfural: a renewable and versatile platform molecule for the synthesis of chemicals and fuels. Energy Environ. Sci., 9(4), 1144-1189.

Mellmer, M.A., Sener, C., Gallo, J.M.R., Luterbacher, J.S., Alonso, D.M., Dumesic, J.A. 2014. Solvent effects in acid-catalyzed biomass conversion reactions. Angew. Chem. Int. Ed., 53(44), 11872-11875. 
Mika, L.s.T., Cséfalvay, E., Németh, A.r. 2017. Catalytic Conversion of Carbohydrates to Initial Platform Chemicals: Chemistry and Sustainability. Chem. Rev.

Mittal, A., Black, S.K., Vinzant, T.B., O’Brien, M., Tucker, M.P., Johnson, D.K. 2017a. Production of furfural from process-relevant biomass-derived pentoses in a biphasic reaction system. ACS Sustainable Chem. Eng., 5(7), 5694-5701.

Mittal, A., Katahira, R., Donohoe, B.S., Pattathil, S., Kandemkavil, S., Reed, M.L., Biddy, M.J., Beckham, G.T.J.A.S.C., Engineering. 2017b. Ammonia pretreatment of corn stover enables facile lignin extraction. ACS Sustainable Chem. Eng., 5(3), 2544-2561.

Modenbach, A.A., Nokes, S.E. 2012. The use of high-solids loadings in biomass pretreatment - a review. Biotechnol. Bioeng., 109(6), 1430-1442.

Moghaddam, L., Rencoret, J., Maliger, V.R., Rackemann, D.W., Harrison, M.D., Gutierrez, A., del Río, J.C., Doherty, W.O. 2017. Structural Characteristics of Bagasse Furfural Residue and Its Lignin Component. An NMR, Py-GC/MS, and FTIR Study. ACS Sustainable Chem. Eng., 5(6), 4846-4855.

Moghaddam, L., Zhang, Z., Wellard, R.M., Bartley, J.P., O'Hara, I.M., Doherty, W.O.S. 2014. Characterisation of lignins isolated from sugarcane bagasse pretreated with acidified ethylene glycol and ionic liquids. Biomass Bioenergy, 70, 498-512.

Mood, S.H., Golfeshan, A.H., Tabatabaei, M., Jouzani, G.S., Najafi, G., Gholami, M., Ardjmand, M. 2013. Lignocellulosic biomass to bioethanol, a comprehensive review with a focus on pretreatment. Renewable Sustainable Energy Rev., 27, 77-93.

Morandeira, L., Álvarez, M.S., Markiewicz, M., Stolte, S., Rodríguez, A., Sanromán, M.Á., Deive, F.J. 2017. Testing True Choline Ionic Liquid Biocompatibility from a Biotechnological Standpoint. ACS Sustainable Chem. Eng., 5(9), 83028309.

Mosier, N., Wyman, C., Dale, B., Elander, R., Lee, Y.Y., Holtzapple, M., Ladisch, M. 2005. Features of promising technologies for pretreatment of lignocellulosic biomass. Bioresour. Technol., 96(6), 673-686.

Moxley, G., Gaspar, A.R., Higgins, D., Xu, H. 2012. Structural changes of corn stover lignin during acid pretreatment. J. Ind. Microbiol. Biotechnol., 39(9), 12891299.

Narron, R.H., Chang, H.-m., Jameel, H., Park, S. 2017. Soluble Lignin Recovered from Biorefinery Pretreatment Hydrolyzate Characterized by LigninCarbohydrate Complexes. ACS Sustainable Chem. Eng., 5(11), 10763-10771.

Nguyen, T.Y., Cai, C.M., Osman, O., Kumar, R., Wyman, C.E. 2016. CELF pretreatment of corn stover boosts ethanol titers and yields from high solids SSF with low enzyme loadings. Green Chem., 18(6), 1581-1589.

Ninomiya, K., Kamide, K., Takahashi, K., Shimizu, N. 2012. Enhanced enzymatic saccharification of kenaf powder after ultrasonic pretreatment in ionic liquids at room temperature. Bioresour. Technol., 103(1), 259-265.

Palmqvist, E., Hahn-Hägerdal, B. 2000. Fermentation of lignocellulosic hydrolysates. II: inhibitors and mechanisms of inhibition. Bioresour. Technol., 74(1), 25-33.

Pandey, K.K., Pitman, A.J. 2004. Examination of the lignin content in a softwood and a hardwood decayed by a brown-rot fungus with the acetyl bromide method 
and Fourier transform infrared spectroscopy. J. Polym. Sci., Part A: Polym. Chem., 42(10), 2340-2346.

Pandey, K.K., Pitman, A.J. 2003. FTIR studies of the changes in wood chemistry following decay by brown-rot and white-rot fungi. Int. Biodeterior. Biodegrad., 52(3), 151-160.

Peleteiro, S., Rivas, S., Alonso, J.L., Santos, V., Parajó, J.C. 2016. Furfural production using ionic liquids: A review. Bioresour. Technol., 202(Supplement C), 181-191.

Phongpreecha, T., Hool, N.C., Stoklosa, R.J., Klett, A.S., Foster, C.E., Bhalla, A., Holmes, D., Thies, M.C., Hodge, D.B. 2017. Predicting lignin depolymerization yields from quantifiable properties using fractionated biorefinery lignins. Green Chem., 19(21), 5131-5143.

Procentese, A., Johnson, E., Orr, V., Garruto Campanile, A., Wood, J.A., Marzocchella, A., Rehmann, L. 2015. Deep eutectic solvent pretreatment and subsequent saccharification of corncob. Bioresour. Technol., 192, 31-36.

Ragauskas, A., Pu, Y., Samuel, R., Jiang, N., Fu, C., Wang, Z.-Y. 2014a. Structural characterization of lignin in wild-type versus COMT down-regulated switchgrass. Front. Energy Res. , 1, 14.

Ragauskas, A.J., Beckham, G.T., Biddy, M.J., Chandra, R., Chen, F., Davis, M.F., Davison, B.H., Dixon, R.A., Gilna, P., Keller, M. 2014b. Lignin valorization: improving lignin processing in the biorefinery. Science, 344(6185), 1246843.

Rahikainen, J.L., Evans, J.D., Mikander, S., Kalliola, A., Puranen, T., Tamminen, T., Marjamaa, K., Kruus, K. 2013. Cellulase-lignin interactions-The role of carbohydrate-binding module and $\mathrm{pH}$ in non-productive binding. Enzyme Microb. Technol., 53(5), 315-321.

Ramachandriya, K.D., Wilkins, M., Atiyeh, H.K., Dunford, N.T., Hiziroglu, S. 2013. Effect of high dry solids loading on enzymatic hydrolysis of acid bisulfite pretreated Eastern redcedar. Bioresour. Technol., 147(Supplement C), 168176.

Renders, T., Van den Bosch, S., Koelewijn, S.-F., Schutyser, W., Sels, B. 2017. Lignin-first biomass fractionation: the advent of active stabilisation strategies. Energy Environ. Sci., 10(7), 1551-1557.

Rinaldi, R., Jastrzebski, R., Clough, M.T., Ralph, J., Kennema, M., Bruijnincx, P.C., Weckhuysen, B.M. 2016. Paving the Way for Lignin Valorisation: Recent Advances in Bioengineering, Biorefining and Catalysis. Angew. Chem. Int. Ed., 55(29), 8164-215.

Rodriguez, A., Salvachúa, D., Katahira, R., Black, B.A., Cleveland, N.S., Reed, M., Smith, H., Baidoo, E.E., Keasling, J.D., Simmons, B.A. 2017. Base-catalyzed depolymerization of solid lignin-rich streams enables microbial conversion. ACS Sustainable Chem. Eng., 5(9), 8171-8180.

Saha, B., Abu-Omar, M.M.J.G.C. 2014. Advances in 5-hydroxymethylfurfural production from biomass in biphasic solvents. Green Chem., 16(1), 24-38.

Samuel, R., Foston, M., Jiang, N., Allison, L., Ragauskas, A.J. 2011. Structural changes in switchgrass lignin and hemicelluloses during pretreatments by NMR analysis. Polym. Degrad. Stab., 96(11), 2002-2009.

Samuel, R., Pu, Y., Raman, B., Ragauskas, A.J. 2010. Structural characterization and comparison of switchgrass ball-milled lignin before and after dilute acid pretreatment. Appl. Biochem. Biotechnol., 162(1), 62-74. 
Sannigrahi, P., Kim, D.H., Jung, S., Ragauskas, A. 2011. Pseudo-lignin and pretreatment chemistry. Energy Environ. Sci., 4(4), 1306-1310.

Saratale, R.G., Shin, H.S., Ghodake, G.S., Kumar, G., Oh, M.K., Saratale, G.D. 2018. Combined effect of inorganic salts with calcium peroxide pretreatment for kenaf core biomass and their utilization for 2, 3-butanediol production. Bioresour. Technol., 258, 26-32.

Sathitsuksanoh, N., Holtman, K.M., Yelle, D.J., Morgan, T., Stavila, V., Pelton, J., Blanch, H., Simmons, B.A., George, A. 2014. Lignin fate and characterization during ionic liquid biomass pretreatment for renewable chemicals and fuels production. Green Chem., 16(3), 1236-1247.

Satlewal, A., Agrawal, R., Bhagia, S., Sangoro, J., Ragauskas, A.J. 2018. Natural deep eutectic solvents for lignocellulosic biomass pretreatment: Recent developments, challenges and novel opportunities. Biotechnol. Adv.

Segal, L., Creely, J., Martin Jr, A., Conrad, C. 1959. An empirical method for estimating the degree of crystallinity of native cellulose using the X-ray diffractometer. Text. Res. J., 29(10), 786-794.

Sen, S., Patil, S., Argyropoulos, D.S. 2015. Thermal properties of lignin in copolymers, blends, and composites: a review. Green Chem., 17(11), 48624887.

Sert, M., Arslanoğlu, A., Ballice, L. 2018. Conversion of sunflower stalk based cellulose to the valuable products using choline chloride based deep eutectic solvents. Renewable Energy, 118, 993-1000.

Sewalt, V., Glasser, W., Beauchemin, K.J.J.o.A., Chemistry, F. 1997. Lignin impact on fiber degradation. 3. Reversal of inhibition of enzymatic hydrolysis by chemical modification of lignin and by additives. Journal of Agricultural and Food Chemistry, 45(5), 1823-1828.

Shuai, L., Amiri, M.T., Questell-Santiago, Y.M., Héroguel, F., Li, Y., Kim, H., Meilan, R., Chapple, C., Ralph, J., Luterbacher, J.S. 2016a. Formaldehyde stabilization facilitates lignin monomer production during biomass depolymerization. Science, 354(6310), 329-333.

Shuai, L., Questell-Santiago, Y.M., Luterbacher, J.S. 2016b. A mild biomass pretreatment using $\gamma$-valerolactone for concentrated sugar production. Green Chem., 18(4), 937-943.

Sluiter, A., Hames, B., Ruiz, R., Scarlata, C., Sluiter, J., Templeton, D. 2006. Determination of Sugars, Byproducts, and Degradation Products in Liquid Fraction Process Samples. National Renewable Energy Laboratory Technical Report NREL/TP-510-42623.

Sluiter, A., Hames, B., Ruiz, R., Scarlata, C., Sluiter, J., Templeton, D., Crocker, D. 2008. Determination of structural carbohydrates and lignin in biomass. Laboratory analytical procedure, 1617, 1-16.

Smith, E.L., Abbott, A.P., Ryder, K.S. 2014. Deep eutectic solvents (DESs) and their applications. Chem. Rev., 114(21), 11060-11082.

Smith, M.D., Mostofian, B., Cheng, X., Petridis, L., Cai, C.M., Wyman, C.E., Smith, J.C.J.G.C. 2016. Cosolvent pretreatment in cellulosic biofuel production: effect of tetrahydrofuran-water on lignin structure and dynamics. Green Chem., 18(5), 1268-1277.

Soares, B., Tavares, D.J., Amaral, J.L., Silvestre, A.J., Freire, C.S., Coutinho, J.o.A.J.A.S.C., Engineering. 2017. Enhanced solubility of lignin monomeric 
model compounds and technical lignins in aqueous solutions of deep eutectic solvents. ACS Sustainable Chem. Eng., 5(5), 4056-4065.

Socha, A.M., Parthasarathi, R., Shi, J., Pattathil, S., Whyte, D., Bergeron, M., George, A., Tran, K., Stavila, V., Venkatachalam, S., Hahn, M.G., Simmons, B.A., Singh, S. 2014. Efficient biomass pretreatment using ionic liquids derived from lignin and hemicellulose. Proc. Natl. Acad. Sci. U.S.A, 111(35), E3587E3595.

Stoklosa, R., Johnston, D., Nghiem, N. 2018. Utilization of Sweet Sorghum Juice for the Production of Astaxanthin as a Biorefinery Co-Product by Phaffia rhodozyma. ACS Sustainable Chem. Eng.

Sturgeon, M.R., Kim, S., Lawrence, K., Paton, R.S., Chmely, S.C., Nimlos, M., Foust, T.D., Beckham, G.T. 2013. A mechanistic investigation of acidcatalyzed cleavage of aryl-ether linkages: Implications for lignin depolymerization in acidic environments. ACS Sustainable Chem. Eng., 2(3), 472-485.

Suhara, H., Kodama, S., Kamei, I., Maekawa, N., Meguro, S. 2012. Screening of selective lignin-degrading basidiomycetes and biological pretreatment for enzymatic hydrolysis of bamboo culms. Int. Biodeterior. Biodegrad., 75, 176180.

Sun, J., Dutta, T., Parthasarathi, R., Kim, K.H., Tolic, N., Chu, R.K., Isern, N.G., Cort, J.R., Simmons, B.A., Singh, S.J.G.C. 2016a. Rapid room temperature solubilization and depolymerization of polymeric lignin at high loadings. Green Chem., 18(22), 6012-6020.

Sun, S., Sun, S., Cao, X., Sun, R. 2016b. The role of pretreatment in improving the enzymatic hydrolysis of lignocellulosic materials. Bioresour. Technol., 199, 49-58.

Sun, S.-N., Li, H.-Y., Cao, X.-F., Xu, F., Sun, R.-C. 2015. Structural variation of eucalyptus lignin in a combination of hydrothermal and alkali treatments. Bioresour. Technol., 176, 296-299.

Sun, Z., Bottari, G., Afanasenko, A., Stuart, M.C., Deuss, P.J., Fridrich, B., Barta, K. 2018. Complete lignocellulose conversion with integrated catalyst recycling yielding valuable aromatics and fuels. Nat. Catal., 1(1), 82.

Tang, X., Zuo, M., Li, Z., Liu, H., Xiong, C., Zeng, X., Sun, Y., Hu, L., Liu, S., Lei, T. 2017. Green processing of lignocellulosic biomass and its derivatives in deep eutectic solvents. ChemSusChem, 10(13), 2696-2706.

Um, J., Kim, D.G., Jung, M.-Y., Saratale, G.D., Oh, M.-K. 2017. Metabolic engineering of Enterobacter aerogenes for 2, 3-butanediol production from sugarcane bagasse hydrolysate. Bioresour. Technol., 245, 1567-1574.

Wan, C., Li, Y. 2012. Fungal pretreatment of lignocellulosic biomass. Biotechnol. $A d v ., 30(6), 1447-1457$.

Wen, J.L., Yuan, T.Q., Sun, S.L., Xu, F., Sun, R.C. 2014. Understanding the chemical transformations of lignin during ionic liquid pretreatment. Green Chem., 16(1), 181-190.

Xia, Q., Liu, Y., Meng, J., Cheng, W., Chen, W., Liu, S., Liu, Y., Li, J., Yu, H. 2018. Multiple hydrogen bond coordination in three-constituent deep eutectic solvents enhances lignin fractionation from biomass. Green Chem., 20(12),2711-2721. 
Xia, S., Baker, G.A., Li, H., Ravula, S., Zhao, H. 2014. Aqueous Ionic Liquids and Deep Eutectic Solvents for Cellulosic Biomass Pretreatment and Saccharification. RSC Adv, 4(21), 10586-10596.

Xie, S., Sun, Q., Pu, Y., Lin, F., Sun, S., Wang, X., Ragauskas, A.J., Yuan, J.S. 2017. Advanced chemical design for efficient lignin bioconversion. ACS Sustainable Chem. Eng., 5(3), 2215-2223.

Xie, S., Yi, C., Qiu, X.J.A.J. 2015. Salting-out of acetone, 1-butanol, and ethanol from dilute aqueous solutions. AlChE J., 61(10), 3470-3478.

Xu, F., Sun, J., Wehrs, M., Kim, K.H., Rau, S.S., Chan, A.M., Simmons, B.A., Mukhopadhyay, A., Singh, S. 2018. Biocompatible choline-based deep eutectic solvents enable one-pot production of cellulosic ethanol. ACS Sustainable Chem. Eng., 6 (7), 8914-8919.

Xu, G.C., Ding, J.C., Han, R.Z., Dong, J.J., Ni, Y. 2016. Enhancing cellulose accessibility of corn stover by deep eutectic solvent pretreatment for butanol fermentation. Bioresour. Technol., 203, 364-9.

Yemiş, O., Mazza, G. 2011. Acid-catalyzed conversion of xylose, xylan and straw into furfural by microwave-assisted reaction. Bioresour. Technol., 102(15), 7371-7378.

Yu, H., Xing, Y., Lei, F., Liu, Z., Liu, Z., Jiang, J. 2014. Improvement of the enzymatic hydrolysis of furfural residues by pretreatment with combined green liquor and ethanol organosolv. Bioresour. Technol., 167, 46-52.

Yuan, T.-Q., Sun, S.-N., Xu, F., Sun, R.-C. 2011. Characterization of lignin structures and lignin-carbohydrate complex (LCC) linkages by quantitative $13 \mathrm{C}$ and $2 \mathrm{D}$ HSQC NMR spectroscopy. J. Agric. Food. Chem., 59(19), 10604-10614.

Zhai, R., Hu, J., Saddler, J.N. 2018. Minimizing cellulase inhibition of whole slurry biomass hydrolysis through the addition of carbocation scavengers during acid-catalyzed pretreatment. Bioresour. Technol., 258, 12-17.

Zhang, C.W., Xia, S.Q., Ma, P.S. 2016a. Facile pretreatment of lignocellulosic biomass using deep eutectic solvents. Bioresour Technol, 219, 1-5.

Zhang, K., Pei, Z., Wang, D. 2016b. Organic solvent pretreatment of lignocellulosic biomass for biofuels and biochemicals: A review. Bioresour. Technol., 199, 21-33.

Zhang, L., Xi, G., Zhang, J., Yu, H., Wang, X. 2017a. Efficient catalytic system for the direct transformation of lignocellulosic biomass to furfural and 5hydroxymethylfurfural. Bioresour. Technol., 224(Supplement C), 656-661.

Zhang, L., Yu, H. 2013. Conversion of xylan and xylose into furfural in biorenewable deep eutectic solvent with trivalent metal chloride added. BioResources, 8(4), 6014-6025.

Zhang, L., Yu, H., Wang, P., Dong, H., Peng, X. 2013. Conversion of xylan, d-xylose and lignocellulosic biomass into furfural using $\mathrm{AlCl} 3$ as catalyst in ionic liquid. Bioresour. Technol., 130, 110-116.

Zhang, L., Yu, H., Yu, H.-B., Chen, Z., Yang, L. 2014. Conversion of xylose and xylan into furfural in biorenewable choline chloride-oxalic acid deep eutectic solvent with the addition of metal chloride. Chin. Chem. Lett., 25(8), 11321136.

Zhang, Q., Vigier, K.D.O., Royer, S., Jérôme, F. 2012. Deep eutectic solvents: syntheses, properties and applications. Chem. Soc. Rev., 41(21), 7108-7146. 
Zhang, X., Bai, Y., Cao, X., Sun, R. 2017b. Pretreatment of Eucalyptus in biphasic system for furfural production and accelerated enzymatic hydrolysis.

Bioresour. Technol., 238, 1-6.

Zhao, X., Liu, D. 2013. Kinetic Modeling and Mechanisms of Acid-Catalyzed Delignification of Sugarcane Bagasse by Aqueous Acetic Acid. Bioenergy Res., 6(2), 436-447.

Zhao, Y., Wang, Y., Zhu, J., Ragauskas, A., Deng, Y. 2008. Enhanced enzymatic hydrolysis of spruce by alkaline pretreatment at low temperature. Biotechnol. Bioeng., 99(6), 1320-1328.

Zhao, Z., Chen, X., Ali, M.F., Abdeltawab, A.A., Yakout, S.M., Yu, G. 2018. Pretreatment of wheat straw using basic ethanolamine-based deep eutectic solvents for improving enzymatic hydrolysis. Bioresour Technol, 263, 325333.

Zheng, Y., Zhao, J., Xu, F., Li, Y. 2014. Pretreatment of lignocellulosic biomass for enhanced biogas production. Prog. Energy Combust. Sci., 42, 35-53.

Zhou, H., Zhang, R.L., Zhan, W., Wang, L.Y., Guo, L.J., Liu, Y. 2016a. High biomass loadings of $40 \mathrm{wt} \%$ for efficient fractionation in biorefineries with an aqueous solvent system without adding adscititious catalyst. Green Chem., 18(22), 6108-6114.

Zhou, S., Xue, Y., Sharma, A., Bai, X. 2016b. Lignin Valorization through Thermochemical Conversion: Comparison of Hardwood, Softwood and Herbaceous Lignin. ACS Sustainable Chem. Eng., 4(12), 6608-6617.

Zuo, M., Le, K., Feng, Y., Xiong, C., Li, Z., Zeng, X., Tang, X., Sun, Y., Lin, L. 2018. An effective pathway for converting carbohydrates to biofuel 5ethoxymethylfurfural via 5-hydroxymethylfurfural with deep eutectic solvents (DESs). Ind. Crops Prod., 112, 18-23.

Zuo, M., Le, K., Li, Z., Jiang, Y., Zeng, X., Tang, X., Sun, Y., Lin, L. 2017. Green process for production of 5-hydroxymethylfurfural from carbohydrates with high purity in deep eutectic solvents. Ind. Crops Prod., 99, 1-6. 


\section{VITA}

Mr. Zhu Chen started his PhD study in 2015 under the supervision of Dr. Wan at the Bioengineering Department, University of Missouri-Columbia. His research is focused on the development of novel green solvent systems for lignocellulosic biomass fractionation, and upgrading of lignocellulosic biomass into platform chemicals via both biological and chemical pathways. During his $\mathrm{PhD}$ study, he published 8 first-authored peer reviewed papers, including 2 review and 6 research papers. 